\title{
Search for non-SM Light Higgs Boson in the $h \rightarrow \gamma \gamma$ Channel
}

\author{
by \\ Alexander Stepanovych Melnitchouk \\ B.Sc., State University of Chernivtsi, Ukraine 1995 \\ Sc.M., Brown University, 1999
}

\begin{abstract}
Thesis
Submitted in partial fulfillment of the requirements for the Degree of Doctor of Philosophy in the Department of Physics at Brown University
\end{abstract}

Providence, Rhode Island May 2004 
Abstract of "Search for non-SM Light Higgs Boson in the $h \rightarrow \gamma \gamma$ Channel " by Alexander Stepanovych Melnitchouk, Ph.D., Brown University, May 2004

We present first results on the search for Higgs Boson with an enhanced branching fraction into photons in the $h \rightarrow \gamma \gamma$ decay channel using recent Run II data collected by the D0 detector at the Fermilab Tevatron proton-antiproton collider. We discuss event selection, backgrounds, analysis optimization, and the limits on the Higgs boson mass obtained in this analysis. 


\section{(C) Copyright}

by

Alexander Stepanovych Melnitchouk

2003 
This dissertation by Alexander Stepanovych Melnitchouk is accepted in its present form by the Department of Physics as satisfying the dissertation requirement for the degree of

Doctor of Philosophy

Date......................

Greg Landsberg, Advisor

Recommended to the Graduate Council

Date......................

Gerald Guralnik, reader

Date.......................

Richard Partridge, reader

Approved by the Graduate Council

Date.......................

Karen Newman

Dean of the Graduate School 
—. He graduated with B.Sc. in Physics (with honors) from the State University of Chernivtsi in 1995. He obtained a M.Sc. degree in Physics from Brown University in 1999. He received exchange program fellowship in 1993 as a junior, from the University of Saskatchewan, Canada. He also received a dissertation fellowship from Brown University in 2003 while he attended the Ph.D. program in the Department of Physics at Brown University. 


\section{Preface and Acknowledgments}

Glory to You for the love of parents, for the faithfulness of friends,

Glory to you for the encounters you arrange for me,

For every condition Your Providence has put me in,

Glory to You for showing me the beauty of the Universe,

Glory to You for all Nature is filled with Your laws,

Glory to You for the dignity of man's labor,

Glory to You for the inventiveness of the human mind,

Glory to You for what You have revealed to us in Your mercy,

Glory to You for what You have hidden from us in Your wisdom.

Fr. Gregory Petrov, "Glory to God for all things"

The fairest thing we can experience is the mysterious.

It is the fundamental emotion which stands at the cradle

of true art and true science.

Albert Einstein, "The World as I See It"

Dear reader, I would like to ask you to excuse the length and a non-traditional style of some paragraphs and individual sentences of this section. It was of help for my attempt to express thankfulness to all who shared with me generously their wonderful gifts during my preparation for and work on this dissertation and a sense of wonder that physics gives us a chance to experience.

Once upon a time, far away on the other side of the Big Ocean, I lived a life of a potential Ph.D candidate. Beech-tree forests in that land were tall and thoughtful, and exceptionally beautiful when summer would just start turning into fall. In the meantime, human life had its joys and challenges at all seasons, and important part of it was food. This was easy for me to appreciate because the 
sweets made by my mom and potato pancakes by both grandmas were the most delicious treats found in my town and many miles around it. And young wine produced by one of my high school buddies in the course of his extra-curriculum activities was of the same quality as the drinks served on a good day on Mount Olympus. The sweets and pancakes were made out of love, tenderness and care, and for that can never be forgotten, while the wine was about teenage solidarity and shared quest for the forbidden things. So a hint was already there that there is much more to reality than can be perceived by senses. Nature of things - it seemed - extended beyond their visible (tastable) self, towards how and why they came to be. Later I realized that physics was a way to explore such an exciting state of affairs, a very special way.

Another part of life over there was work. My dad taught me that work can be very enjoyable experience as long as you do it wholeheartedly, and especially if you could share it with other human beings, the kind of lessons that to this day mean very much to me. One day he told me also about the microscopic world. Its inhabitants, he said, were cute and tiny, their behaviour unpredictable, and yet one could understand them in terms of probabilities and symmetries. In that world miracles happen so abundantly that sometimes they would spill over to our side, where keen eyes and modern equipment could see them. I learned that when two electrons, each wandering about the lattice on its individual business, decide to stick together, from that moment their journey will be nothing like before: even the neighbours that used to give them hard time turn into a special bond between the two of them [1]. And that among snowflakes, beautiful and rich in patterns as they seem, there was hardly ever one with the five-fold rotational symmetry. Moreover, diamonds could be turned blue [2] if on the top of the band energy structure one produces enough local states (by knocking charges out of their place into inter-atomic space with a neutron beam) to change the macroscopic optical properties. However, if the blue diamonds happen to be out of fashion at the time, you could shake the interstitial charges back home by warming the crystal up and thus restore original energy level structure and color.

All this was fascinating and at Brown University this fascination continued, especially when I learned about modern particle physics. I would like to thank professor Rich Partridge for welcoming 
me and introducing me to the Experimental High Energy group, professor Dave Cutts for his support, and professor Greg Landsberg for taking me as his advisee, and helping me to make the transition from my previous focus on theoretical work to experimental research as smooth as I could possibly wish.

The research had to be conducted in the part of the country where beautiful patches of prairies lay here and there. As nights came down on them gently, the firebugs crawled out from underneath the grass to start their sparkling dance in the air. So beautiful and inspiring it was that even the oak groves stopped whispering and watched it enchantedly from nearby. And up in the sky geese were travelling back and forth in perfect spatial configurations learned from a zoology textbook. In the middle of that area great rings were built under the earth. They were called the rings of power for hardly any of mortal men could help coming back after visiting them once. Some would come every now and then, some would do it every week, others would settle there for couple of years and still others - for life. Even on the other side of the Big Ocean their power was felt and attracted folks from over there. Undoubtedly it was a power of good because people were very happy to be there despite the fact that their daily lives revolved around problem-facing and problem-solving. It was a lot of fun and a privilege to come to this place and to work on the DØ detector upgrade for RunII. I would like to thank Robert Wilson for the Fermilab and Paul Granis for the DØ experiment, as well as all people who worked diligently on the construction of these research facilities.

Thanks to Greg's wisdom and broad knowledge of the field, I was able to combine my interest in Higgs boson search with the skills that I already obtained working on identification of photons. This topic attracted me because of the role the Higgs field may play in current theory, namely explaining how mass originates. Our very existence seems impossible without matter - Nature around us, as we see it, is made of matter, and we have material bodies which consist of fermions to which the Higgs boson possibly gives mass. Not only that, but also it is the material carriers that most often bring to us things that transcend the material such as truth, music, or love, as well as some beautiful ideas, like those of the theory of Groups and Representations. We learn that this theory was born less than two centuries ago when Evarest Galua first came up with it. However, it may be interesting to 
think that it must have been around for at least a dozen of billion of years - according to its rules the hadrons were formed out of quarks shortly after the Big Bang.

I would like to thank all people who gave me an opportunity to work in the field that is about such exciting questions and helped me to contribute with my Ph.D. research. First of all - my advisor for guiding me all along, for diverse and thorough training in methods and ideas of experimental high energy physics, and, above all, for helping me to grow as an individual, for showing me an excellent example of advisorship, which I will definitely try to follow myself when I get to the point of passing my knowledge and experience to others, and for making my life as a graduate student smooth and happy, and at the same time filled with intense meaningful and exciting work!

I would also like to thank Yuri Gershtein for being my mentor at Fermilab, for introducing me to the world of histograms and ntuples, for finding bugs in my code when I myself could not find them, and for being a cool person to work with.

I was also fortunate to get to know Stelios Kesisoglou. As he came from the land where people always found great delight in arts and geometry, he carried in him a sense of beauty and harmony, kind and gentle personality, and in his memory fifteen different ways to prove some important fact about the sides of a right triangle. Not that it was a particularly controversial issue calling for much proof and debate (in fact for last two and a half thousand years it was generally agreed upon). Nevertheless in case such controversies may still arise this man was prepared to put all of the fifteen proofs on the table. Two of us shared joys, disappointments, DØ software tricks and made fun of one another on a regular basis. I am very indebted to Stelios for his support and help during the years that we shared at $\mathrm{D} \varnothing$. To him also goes credit for discovering accidentally that silicon ladder detectors despite their macroscopic size were quantum-mechanical objects (after he measured geometrical parameters of a ladder on a non-contact measuring machine, the ladder's properties would often change in a very particular way from what they used to be prior to the measurement).

I would like to thank DØ Higgs and EMID group conveners - Ron Madaras, Michel Jaffre, Volker Buescher, Ursula Bassler, Boaz Klima, Meenakshi Narain, John Hobbs, and Avto Kharchilava for their help and advice on the issues of analysis and particle identification. I am also thankful to 
my roommates Gregor Geurkov for telling me about Fermilab back in Brown and for getting me interested in it, and to Richard Genik II for being my tireless teacher of DØ when I was a newcomer. I appreciated a lot a company of Erick Kajfasz, Jim Fast, Ryan Hickey, Wayne Schmitt, and other people when working on Silicon Detector assembly together with Greg, Yuri, and Stelios. I thank Abid Patwa for providing me a thorough collection of detector plots for the dissertation.

And last but not least - my thanks to all people whose impact on my dissertation was less direct yet extremely important : my family, friends and teachers, classmates and colleagues, brothers and sisters in faith back in Ukraine and in the US.

I was glad to be a part of the effort of large HEP community that brings not only fundamental physics discoveries but also technological advancements from which people outside physics could benefit; in particular, all people of this country, whose daily work, generosity, and open mind made it possible for me to do research in the world's leading science center and to receive graduate education together with many other lessons and blessings of life. 


\section{Dedication}

I would like to dedicate this work to my parents Stepan and Lidia. 


\section{Contents}

Preface and Acknowledgments $\quad$ iv

$\begin{array}{ll}\text { Dedication } & \text { ix }\end{array}$

1 Introduction 1

2 Theory and Phenomenology 4

2.1 Standard Model . . . . . . . . . . . . . . . . . . . . . . . . 4

2.1.1 Particles of the Standard Model _. . . . . . . . . . . . . . . . 4

2.1 .2 Local Gauge Invariance $\ldots \ldots \ldots \ldots$

2.1.3 Electroweak Symmetry Breaking . . . . . . . . . . . . . . . 6

2.1.4 Standard Model Lagrangian . . . . . . . . . . . . . . . . . . . 7

2.1.5 Cross Sections and Decay Widths . . . . . . . . . . . . . . . . . . . 10

2.2 Beyond the Standard Model . . . . . . . . . . . . . . . . . . . . . . 11

2.2.1 Why the Standard Model Needs to be Extended . . . . . . . . . . . . . . 11

$2.2 .2 \quad$ Supersymmetry . . . . . . . . . . . . . . . . . . . . . . 12

2.2 .3 Strong Dynamics . . . . . . . . . . . . . . . . . . . . . . 13

2.2 .4 Extra Dimensions . . . . . . . . . . . . . . . . . . . . . . . 14

2.3 Higgs Production and Decay $\ldots \ldots \ldots \ldots \ldots \ldots \ldots$

2.3.1 Higgs Production at the Tevatron . . . . . . . . . . . . . . . . 15

$2.3 .2 \quad$ Higgs Decays . . . . . . . . . . . . . . . . . . . . . . . . . 17 
2.4 Constraints on the Higgs Mass - Previous Results . . . . . . . . . . . . . . 20

3 Fermilab and the DØDetector $\quad 21$

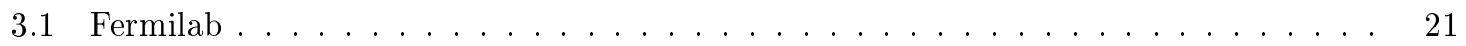

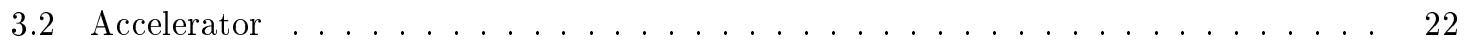

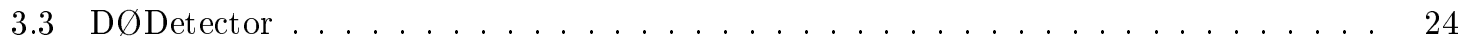

3.4 Coordinate System and Useful Variables . . . . . . . . . . . . . . . . 25

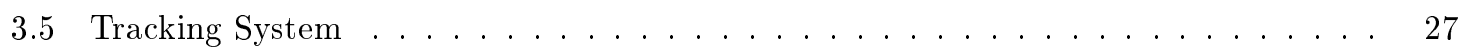

3.5.1 Silicon Tracker . . . . . . . . . . . . . . . . . . . . . 27

3.5 .2 Central Fiber Tracker . . . . . . . . . . . . . . . . . . . . . 32

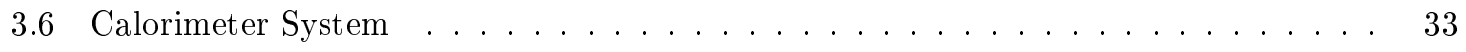

3.6 .1 Calorimetry Basics . . . . . . . . . . . . . . . . . . . 33

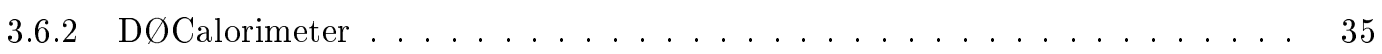

3.7 Intercryostat and Massless Gap Detectors . . . . . . . . . . . . . . . . . . . 40

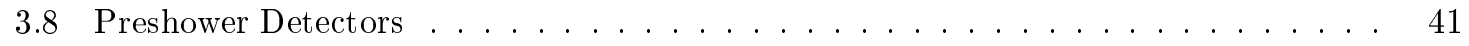

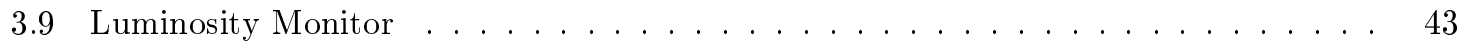

3.10 Muon System . . . . . . . . . . . . . . . . . . . . . 44

3.11 Forward Proton Detector $\ldots \ldots \ldots \ldots \ldots$

3.12 Trigger and Data Acquisition Systems f . . . . . . . . . . . . . . 46

4 Photon Identification $\quad 51$

4.1 Electromagnetic Showers . . . . . . . . . . . . . . . . . . . 52

4.2 Hadronic Showers . . . . . . . . . . . . . . . . . . . . . . 55

4.3 Electromagnetic and Hadronic Shower Shape . . . . . . . . . . . . . . 56

4.3.1 Longitudinal Extent and Energy Loss . . . . . . . . . . . . . . . . . 57

4.3.2 Transverse Shower Shape . . . . . . . . . . . . . . . . . . . 58

4.4 Cluster Reconstruction and EMID variables $\ldots \ldots \ldots \ldots \ldots \ldots$

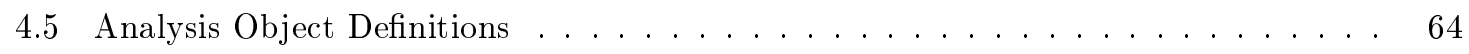


4.6 Misidentification Rate . . . . . . . . . . . . . . . . . . . 64

4.6.1 What is a 'Fake Photon'? . . . . . . . . . . . . . . . . 65

4.6.2 Definition of Photon/Electron Fake Rate . . . . . . . . . . . . . . 65

4.6.3 Fake Rate Measurement Sample . . . . . . . . . . . . . . . . . . . 66

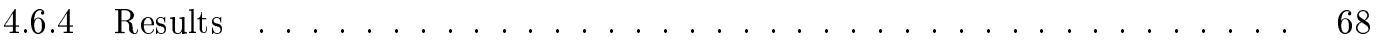

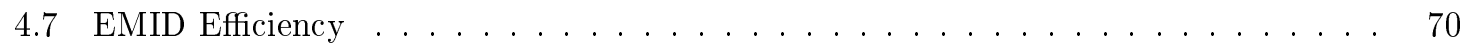

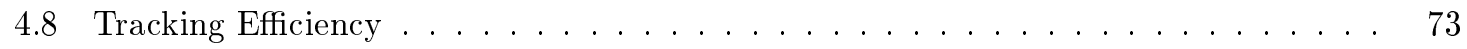

$\begin{array}{llr}5 & \text { Analysis } & 78\end{array}$

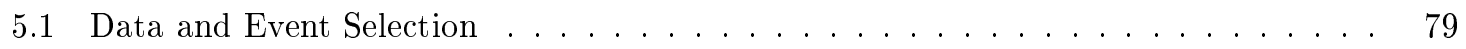

$5.1 .1 \quad$ Dataset . . . . . . . . . . . . . . . . . . . . 79

5.1 .2 Di-LooseEM Data Sample . . . . . . . . . . . . . . . . . . . . . . . 79

$5.1 .3 \quad$ Final Data Sample . . . . . . . . . . . . . . . . . . . . 80

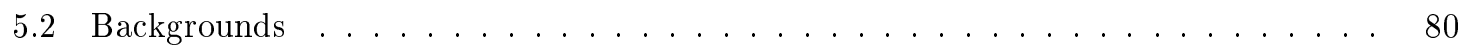

5.2 .1 Sources of Background . . . . . . . . . . . . . . . . 80

5.2 .2 Predicting the Drell-Yan Background . . . . . . . . . . . . . . . 80

5.2 .3 Predicting QCD $\gamma \gamma$ Background . . . . . . . . . . . . . 84

5.2.4 Summary of Background Estimates . . . . . . . . . . . . . . . 84

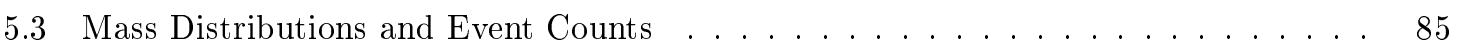

5.4 Monte Carlo Signal Samples . . . . . . . . . . . . . . . . . . . . . . . . . . 89

5.5 Event Topology and EM Efficiency . . . . . . . . . . . . . . . . . . . 89

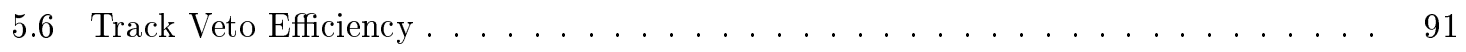

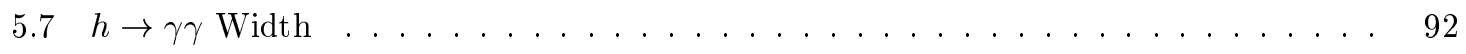

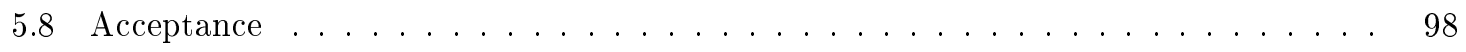

5.8.1 Mass Window Cut Acceptance . . . . . . . . . . . . . . . . . . 99

5.8.2 Fiducial and $p_{T}$ Acceptance $\ldots \ldots \ldots \ldots \ldots$. . . . . . . . 99

5.9 Event Counts . . . . . . . . . . . . . . . . . . . . . . . . . . 104

5.10 Analysis Optimization . . . . . . . . . . . . . . . . . 105 
5.10 .1 Outline of Optimization . . . . . . . . . . . . . . . . . 105

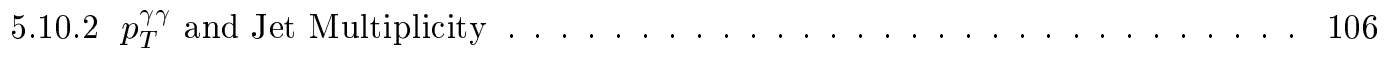

5.10 .3 Event Counts After $p_{T}^{\gamma \gamma}$ cut $\ldots \ldots \ldots \ldots$. . . . . . . . . 122

5.10.4 Other Kinematic Variables : $d R^{\gamma \gamma}(\eta, \phi),(E 1-E 2) /(E 1+E 2),\left|\cos \theta^{*}\right| \ldots 128$

5.10 .5 Neural Network Studies . . . . . . . . . . . . . . . . . . . . . 150

5.10 .6 Conclusion on Optimization . . . . . . . . . . . . . . . . . 158

6 Higgs Cross Section Limits $\quad 159$

6.1 Bayesian Approach . . . . . . . . . . . . . . . . . . . . . . . . 159

$6.1 .1 \quad$ Brief Overview . . . . . . . . . . . . . . . . . 159

$6.1 .2 \quad h \rightarrow \gamma \gamma$ applications . . . . . . . . . . . . . . . 161

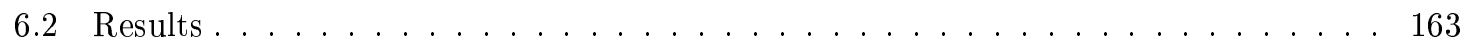

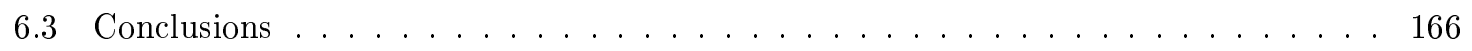

$\begin{array}{llr}\text { A Appendix A } & 168\end{array}$ 


\section{List of Tables}

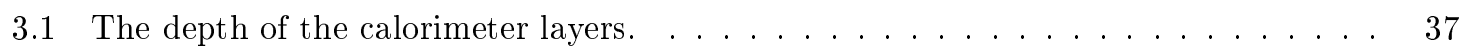

4.1 Radiation length $X_{0}$, absorption length $\lambda$, and critical energy $\epsilon$ for several materials. $\quad 56$

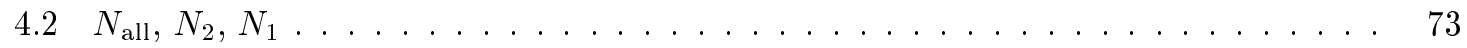

5.1 Track veto efficiency (per $h \rightarrow \gamma \gamma$ event) for the Fermiophobic Higgs. . . . . . . . 92

5.2 Track veto efficiency (per $h \rightarrow \gamma \gamma$ event) for the Topcolor Higgs. . . . . . . . . . 92

5.3 Mass window acceptance for Fermiophobic Higgs . . . . . . . . . . . . . . . . 100

5.4 Mass window acceptance for Topcolor Higgs . . . . . . . . . . . . . . . . . . 100

5.5 Fiducial and $p_{T}$ acceptance for Fermiophobic Higgs. . . . . . . . . . . . . . . 104

5.6 Fiducial and $p_{T}$ acceptance for Topcolor Higgs. . . . . . . . . . . . . . . . . 104

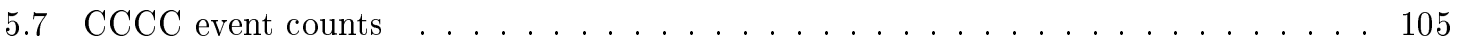

5.8 CCCC event count, expected signal and backgrounds f . . . . . . . . . . . . 122

5.9 Predicted background and observed number of events as a function of the mass window

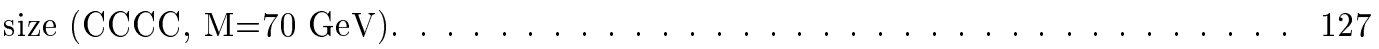

5.10 Predicted background and number of observed events as a function of a mass window $\operatorname{size}(\mathrm{CCEC}, \mathrm{M}=60 \mathrm{GeV}) \ldots \ldots \ldots \ldots \ldots \ldots \ldots \ldots \ldots$

$6.195 \% C L$ upper limits on Higgs cross-section. Theoretical cross-sections . . . . . . . . 165

A.1 Comparison of tracking efficiencies obtained via different formulas and different background fit functions. . . . . . . . . . . . . . . . . 168 


\section{List of Figures}

2.1 Higgs production in the $p \bar{p}$ collision via gluon fusion. . . . . . . . . . . . 16

2.2 Higgs production in the $p \bar{p}$ collision via $\mathrm{W} / \mathrm{Z}$ associated production (top diagram) and in $\mathrm{W} / \mathrm{Z}$ fusion $($ bottom diagram $) . \ldots \ldots \ldots \ldots \ldots$

2.3 Higgs production cross section at the Tevatron as a function of Higgs mass [23, 24, 25]. 17

2.4 SM Higgs decay branching fractions. The $\gamma \gamma$ branching fraction $\left(\sim 10^{-3}-10^{-4}\right)$ is

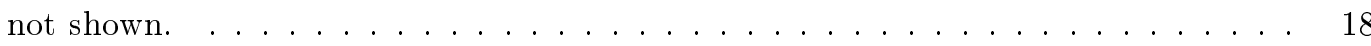

2.5 Diphoton Higgs decays. Top diagram: W-loop decay, bottom diagram: top-quark

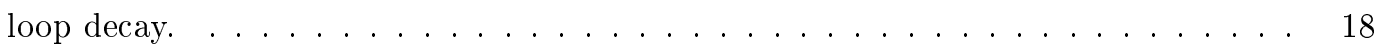

2.6 Examples of enhanced diphoton decays of the Higgs. Lines indicate (top to bottom): fermiophobic (bosonic) Higgs $h_{b h}^{0}$; electroweak Higgs $h_{e w}^{0}$ with zero couplings to the top and bottom quarks; Higgs that couples only to up-type fermions $h_{u}^{0}$; the Standard Model Higgs. . . . . . . . . . . . . . . . . . . . . . . . . . .

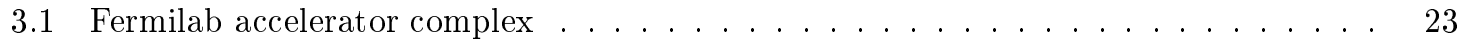

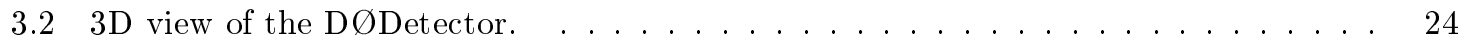

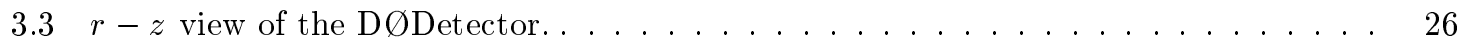

3.4 DØtracking system (the quadrant $r-z$ view) (Adapted from [51]) $\ldots \ldots \ldots \ldots$

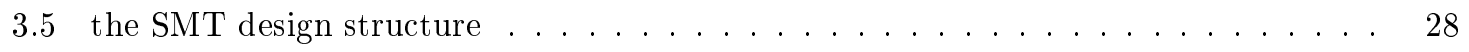

3.6 DØ3-chip ladder photograph. (Adapted from [55]) $\ldots \ldots \ldots \ldots$

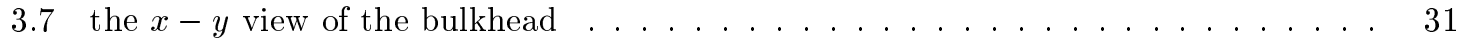

3.8 A bulkhead with two ladders installed $\ldots \ldots \ldots \ldots \ldots \ldots \ldots \ldots \ldots$ 
3.9 the $r-z$ view of the scintillating fiber tracker with the end view of the fibers. . . .

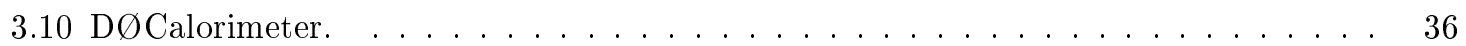

3.11 Pseudo-projective geometry of the DøCalorimeter. . . . . . . . . . . . . . 38

3.12 Calorimeter unit cell . . . . . . . . . . . . . . . . . . . . . . . 39

3.13 Simplified calorimeter data flow diagram. . . . . . . . . . . . . . . . 39

3.14 Full calorimeter data flow diagram. . . . . . . . . . . . . . . . . . . . 40

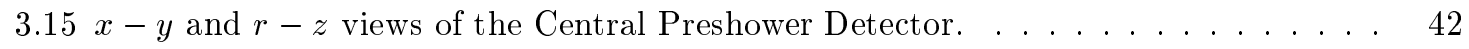

3.16 Location of Forward Preshower Detector in the DØDetector . . . . . . . . . . . . . . 42

3.17 DøLuminosity Monitor. . . . . . . . . . . . . . . . . . . . 43

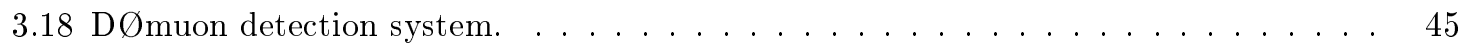

3.19 DØtrigger scheme layout and typical trigger rates. . . . . . . . . . . . . . 47

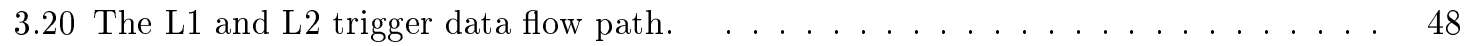

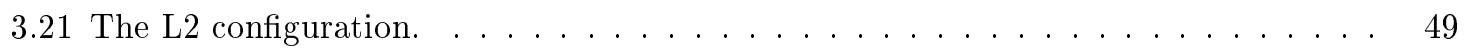

3.22 The L3/DAQ system layout. . . . . . . . . . . . . . . . . . . . 49

4.1 Fractional energy loss per radiation length in lead as a function of the electron or positron energy. At high energies bremsstrahlung dominates. . . . . . . . . . . 52

4.2 Total photon cross sections as a function of energy in carbon and lead, showing contributions of different processes. At high energies pair production dominates $\left(\mathcal{K}_{n u c}\right.$ and $\mathcal{K}_{e}$ denote pair production in nuclear and electron fields, respectively). . . . . 53

4.3 $30 \mathrm{GeV}$ electron cascade induced in iron (EGS4 simulation). The histogram shows fractional energy deposition per radiation length, and the curve is a gamma-function fit to the distribution. Circles indicate the number of electrons with total energy greater than $1.5 \mathrm{MeV}$ crossing planes at $X_{0} / 2$ intervals (scale on the right) and the squares are the number of photons with $E \geq 1.5 \mathrm{MeV}$ crossing the planes (scaled down to have the same area as the electron distribution) $\ldots \ldots \ldots \ldots$. . . 57

4.4 Cluster reconstruction $\eta-\phi$ window contours. . . . . . . . . . . . . . . 60

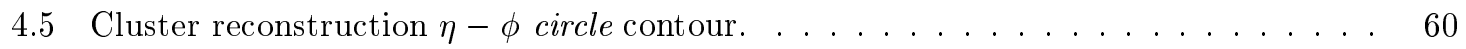


4.6 pictorial representation of the EisoTot, EisoCore and iso EMID varialbes. . . . . .

4.7 pictorial representation of cluster energy $(E)$, hadronic energy of a cluster $(H A)$, and EM energy fraction $(E M f r a c)$ EMID varialbes $\ldots . . . . . . . . . .$.

4.8 Monte-Carlo example of the distributions of Isolation (iso), EMFraction (EM frac), and $H-$ Matrix $\chi^{2}$ EMID variables. Red: Single Photons; Black: Hadronic jets that passed $i s o<0.1$ and EMfrac $>0.9$ (Probability for a hadronic jet to pass these cuts is $\approx 0.01)$. In this example a tighter Isolation cut of 0.1 was used. In real data the discrimination is expected to be somewhat worse since the H-Matrix was trained on the Monte-Carlo sample that does not reproduce the data exactly. . . . . . . .

4.9 The $p_{T}$ distribution for the generator level Photons in the decay of a $120 \mathrm{GeV}$ Topcolor Higgs. Leading (in $p_{T}$ ) Photon shown with the diagonal hatching, trailing Photon shown with the cross-hatching. .....................

4.10 The $p_{T}$ distributions and fake rates vs $p_{T}$ in the Central Calorimeter (CC). Top row left $-p_{T}$ distributions of Loose EM objects (open histogram), Photons (light hatched), and Electrons (dark hatched) in the EM_LO subsample; Top row middle $p_{T}$ distributions for the Loose EM objects (open histogram), Photons (light hatched) and Electrons (dark hatched) in the EM_HI subsample; Middle row left - Photon fake rate for the EM_LO subsample; Bottom row left - Electron fake rate for the EM_LO subsample; Middle row middle - Photon fake rate for the EM_HI subsample; Bottom row middle - Electron fake rate for the EM_HI subsample; Middle row right - Photon fake rate for the combined sample; Bottom row right - Electron fake rate for the

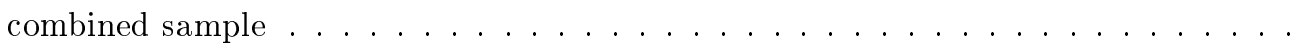


4.11 The $p_{T}$ distributions and fake rates vs $p_{T}$ in the End Cap Calorimeter (EC). Top row left $-p_{T}$ distributions of Loose EM objects (open histogram), Photons (light hatched), and Electrons (dark hatched) in the EM_LO subsample; Top row middle $p_{T}$ distributions for the Loose EM objects (open histogram), Photons (light hatched) and Electrons (dark hatched) in the EM_HI subsample; Middle row left - Photon fake rate for the EM_LO subsample; Bottom row left - Electron fake rate for the EM_LO subsample; Middle row middle - Photon fake rate for the EM_HI subsample; Bottom row middle - Electron fake rate for the EM_HI subsample; Middle row right - Photon fake rate for the combined sample; Bottom row right - Electron fake rate for the combined sample $\ldots \ldots \ldots \ldots \ldots \ldots \ldots \ldots \ldots \ldots \ldots$

4.12 CCCC diphoton mass distributions (in $\mathrm{GeV}$ ) for the CC EMID Efficiency measurement. Left column corresponds to the case when the tag object is required to have a track match, whereas in the right column uses the calorimeter based cut $($ Isolation $<0.015)$. Top plots shows the denominator mass distributions i.e. when a cut is applied to the tag object only. Bottom plots show numerator mass distributions i.e. when $(\mathrm{EM}) \mathrm{ID}$ cut is applied to the probe object. $\ldots \ldots \ldots \ldots \ldots$

4.13 CCEC diphoton mass distributions (in $\mathrm{GeV}$ ) for the EC EMID Efficiency measurement. Left column corresponds to the case when the tag object is required to have a track match, whereas in the right column uses the calorimeter based cut $($ Isolation $<0.015)$. Top plots shows the denominator mass distributions i.e. when a cut is applied to the tag object only. Bottom plots show numerator mass distributions i.e. when $(\mathrm{EM}) \mathrm{ID}$ cut is applied to the probe object. $\ldots \ldots \ldots \ldots$

4.14 di-EM mass distributions for Tracking Efficiency measurement in CCCC events. The top plot shows the mass spectrum and the fit for the whole sample; the middle plot corresponds to the events with exactly one track match; the bottom plot shows the events in which both EM objects are matched to tracks. Linear function was used to

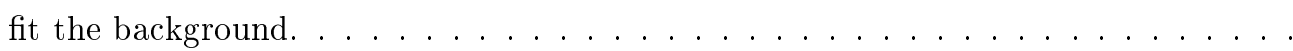


4.15 di-EM mass distributions for Tracking Efficiency measurement in CCEC events. The top plot shows the mass spectrum and the fit for the whole sample; the middle plot corresponds to the events with exactly one track match; the bottom plot shows the events in which both EM objects are matched to tracks. Linear function was used to fit the background. . . . . . . . . . . . . . . .

4.16 di-EM mass distributions for Tracking Efficiency measurement in ECEC events. The top plot shows the mass spectrum and the fit for the whole sample; the middle plot corresponds to the events with exactly one track match; the bottom plot shows the events in which both EM objects are matched to tracks. Linear function was used to

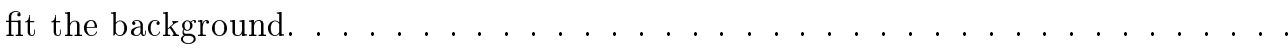

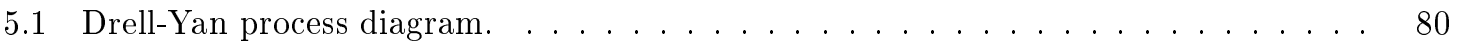

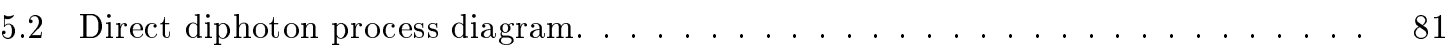

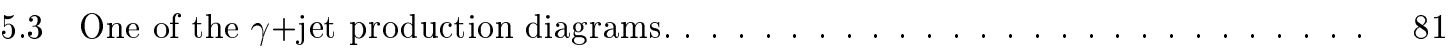

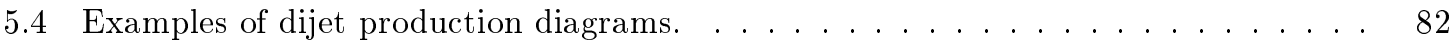

5.5 Distributions in the $\gamma \gamma$ invariant mass and event counts for different event topologies. Logarithmic vertical scale is used. Points $-\gamma \gamma$ spectrum observed in data, solid line - total SM background prediction, dotted line - QCD background, dashed line -

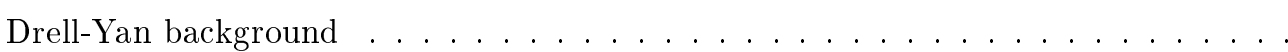

5.6 Same distributions as in previous Figure $(\gamma \gamma$ invariant mass $)$ are shown with liner vertical scale. Points $-\gamma \gamma$ spectrum observed in data, solid line - total SM background prediction, dotted line - QCD background, dashed line - Drell-Yan background . . .

5.7 Same distributions as in previous Figure ( $\gamma \gamma$ invariant mass) are shown for the subsample of data in which bad JET/Missing $E_{T}$ runs are removed. No significant difference with the full sample is observed. Points $-\gamma \gamma$ spectrum observed in data, solid line - total SM background prediction, dotted line - QCD background, dashed line - Drell-Yan background $\ldots \ldots \ldots \ldots \ldots \ldots$ 
5.8 CCCC Mass distributions for the measurement of $\epsilon(C C)$. Left column corresponds to the case when the tag object is required to have a track match, whereas in the right column calorimeter based cut $($ Isolation $<0.006)$ is applied to the tag object. Top plots show the denominator mass distributions, i.e. when the cut is applied on the tag object only. Bottom plots show the numerator mass distributions, i.e. when the (EM) ID cut is applied to the probe object. $\ldots \ldots \ldots \ldots \ldots$

5.9 CCEC Mass distributions for measurement of $\epsilon(E C)$. Left column corresponds to the case when the tag object is required to have a track match, whereas in the right column calorimeter based cut $($ Isolation $<0.006)$ is applied to the tag object. Top plots show the denominator mass distributions, i.e. when the cut is applied on the tag object only. Bottom plots show the numerator mass distributions, i.e. when the (EM) ID cut is applied to the probe object. . . . . . . . . . . . . . 91

5.10 Fermiophobic $h \rightarrow \gamma \gamma$ mass peak in CCCC in reconstructed MC. . . . . . . . 93

5.11 Fermiophobic $h \rightarrow \gamma \gamma$ mass peak in CCEC in reconstructed MC. . . . . . . . . 94

5.12 Topcolor $h \rightarrow \gamma \gamma$ mass peak in CCCC in reconstructed MC. . . . . . . . . . 95

5.13 Topcolor $h \rightarrow \gamma \gamma$ mass peak in reconstructed MC . . . . . . . . . . 96

$5.14 Z \rightarrow e e$ mass peak in CCCC for reconstructed MC. We use only calorimetric energy and direction measurements to calculate the invariant mass. . . . . . . . . . . . 97

$5.15 Z \rightarrow e e$ mass peak in CCCC for the data. We use only calorimetric energy and direction measurements to calculate the invariant mass. . . . . . . . . . . .

$5.16 Z \rightarrow e e$ mass peak in CCEC for reconstructed MC. We use only calorimetric energy and direction measurements to calculate the invariant mass. . . . . . . . . . . 98

$5.17 Z \rightarrow e e$ mass peak in CCEC for the data. We use only calorimetric energy and direction measurements to calculate the invariant mass. . . . . . . . . . . . . . . 99

$5.18 Z \rightarrow e e$ mass peak in the CCCC events for smeared generator-level MC. . . . . . . 101

$5.19 Z \rightarrow e e$ mass peak in the CCCC events for the data . . . . . . . . . . . . 102

$5.20 Z \rightarrow e e$ mass peak in the CCEC events for smeared generator-level MC. . . . . . . . 102 
$5.21 Z \rightarrow e e$ mass peak in the CCEC events for the data. . . . . . . . . . . . 103

5.22 Jet multiplicity for the signal (dashed line) and the background (solid line). $\quad \ldots \quad \ldots 107$

5.23 Relative uncertainty in jet $p_{T}$ due to JES error as a function of jet $\eta$ for $\mathrm{M}=120 \mathrm{GeV}$ Fermiophobic Higgs. . . . . . . . . . . . . . . . . . . . . . . . 108

5.24 Relative uncertainty on the jet PT due to JES error as a function of jet $\eta$ for the di-EM data. . . . . . . . . . . . . . . . . . . . . . 108

5.25 Jet multiplicity (number of jets with $p_{T}>30 \mathrm{GeV}$ ) for the $\mathrm{M}=120 \mathrm{GeV}$ Fermiophobic Higgs. Black line corresponds to JES corrected jets while dotted and dashed lines show $J E S+\sigma$ and $J E S-\sigma$ corrected jets respectively . . . . . . . . . . . . . 109

5.26 Jet multiplicity (number of jets with $p_{T}>30 \mathrm{GeV}$ )in data. Solid line corresponds to JES corrected jets while dotted and dashed line show $J E S+\sigma$ and $J E S-\sigma$ corrected jets respectively . . . . . . . . . . . . . . . . . . . 109

$5.27 p_{T}^{\gamma \gamma}$ distribution for signal (dashed line) and background (solid line). No jet multiplicity cut is applied. . . . . . . . . . . . . . . . . . . 110

$5.28 p_{T}^{\gamma \gamma}$ distribution for signal (dashed line) and background (solid line) for the case when at least one jet with $p_{T}>30 \mathrm{GeV}$ is required to be present in the event $\ldots \ldots \ldots 111$

5.29 Significance as a function of the $p_{T}^{\gamma \gamma}$ cut. Solid curve: no jets are used in the analysis; red curve : at least one jet with $p_{T}>30 \mathrm{GeV}$ is required to be present in the event. Straight line shows the theoretical NLO Fermiophobic Higgs cross-section value (Assuming $B(\gamma \gamma)=1) \ldots \ldots \ldots \ldots \ldots \ldots \ldots \ldots$

5.30 Fermiophobic Higgs CCCC $p_{T}^{\gamma \gamma}$ distributions. Dashed line - signal, solid line - background.

5.31 Fermiophobic Higgs CCEC $p_{T}^{\gamma \gamma}$ distributions. Dashed line - signal, solid line - background. . . . . . . . . . . . . . . . . . . . . 115

5.32 Topcolor Higgs CCCC $p_{T}^{\gamma \gamma}$ distributions. Dashed line - signal, solid line - background. 116

5.33 Topcolor Higgs CCEC $p_{T}^{\gamma \gamma}$ distributions. Dashed line - signal, solid line - background. 117 5.34 Fermiophobic Higgs CCCC Significance vs. $p_{T}^{\gamma \gamma} \ldots \ldots \ldots \ldots \ldots \ldots$ 
5.35 Fermiophobic Higgs CCEC Significance vs. $p_{T}^{\gamma \gamma} . \ldots \ldots \ldots \ldots \ldots$

5.36 Topcolor Higgs CCCC Significance vs. $p_{T}^{\gamma \gamma} \ldots \ldots \ldots \ldots \ldots \ldots \ldots$

5.37 Topcolor Higgs CCEC Significance vs. $p_{T}^{\gamma \gamma} \ldots \ldots \ldots \ldots \ldots \ldots \ldots \ldots$

5.38 Studying background/data discrepancy for $\mathrm{M}=70 \mathrm{GeV}$ CCCC Fermiophobic Higgs.

Top plots show $\Delta \phi$ between the Missing Et and a Jet: top left plot corresponds to the leading jet, top right plot corresponds to the trailing jet. Filled histogram shows observed events after $p_{T}^{\gamma \gamma}$ cut; open histogram corresponds to the case when $p_{T}^{\gamma \gamma}$ cut is removed. Middle plots show $\Delta \phi$ of the top plots vs. Missing Et. Bottom left plot shows Cluster Transverse Mass distribution; bottom right plot shows $d R(\eta, \phi)$ separation between two photons (a photon and potential $W \rightarrow e \nu$ electron). . . . .

5.39 Studying background/data disrcepancy for $\mathrm{M}=60 \mathrm{GeV}$ CCEC Fermiophobic Higgs. Top plots show $\Delta \phi$ between the Missing Et and a Jet: top left plot corresponds to the leading jet, top right plot corresponds to the trailing jet. Filled histogram shows observed events after $p_{T}^{\gamma \gamma}$ cut; open histogram corresponds to the case when $p_{T}^{\gamma \gamma}$ cut is removed. Middle plots show $\Delta \phi$ of the top plots vs. Missing Et. Bottom left plot shows Cluster Transverse Mass distribution; bottom right plot shows $d R(\eta, \phi)$ separation between two photons (a photon and potential $W \rightarrow e \nu$ electron). . . .

5.40 CCCC $\gamma \gamma$ invariant mass distribution for the QCD background after $p_{T}^{\gamma \gamma}$ cut is applied. Dashed and dotted line histograms show two of the QCD background terms as described by equation ??. solid shows total QCD background, points show data. . .

5.41 CCEC $\gamma \gamma$ invariant mass distribution for the QCD background after $p_{T}^{\gamma \gamma}$ cut is applied. Dashed and dotted line histograms show two of the QCD background terms as described by equation ??. solid line shows total QCD background, points show data. 126

5.42 Fermiophobic Higgs CCCC $d R^{\gamma \gamma}(\eta, \phi)$ after the $p_{T}^{\gamma \gamma}$ cut. Dashed line - signal, solid line - background. . . . . . . . . . . . . . . . . . . . . . . 129

5.43 Fermiophobic Higgs CCEC $d R^{\gamma \gamma}(\eta, \phi)$ after the $p_{T}^{\gamma \gamma}$ cut. Dashed line - signal, solid line - background. . . . . . . . . . . . . . . . . . . 
5.44 Topcolor Higgs CCCC $d R^{\gamma \gamma}(\eta, \phi)$ after the $p_{T}^{\gamma \gamma}$ cut. Dashed line - signal, solid line -

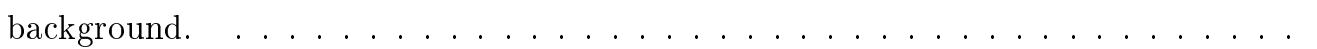

5.45 Topcolor Higgs CCEC $d R^{\gamma \gamma}(\eta, \phi)$ after the $p_{T}^{\gamma \gamma}$ cut. Dashed line - signal, solid line background.

5.46 Fermiophobic Higgs CCCC Significance vs. $d R^{\gamma \gamma}(\eta, \phi)$ after the $p_{T}^{\gamma \gamma}$ cut is applied.

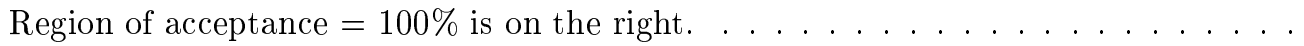

5.47 Fermiophobic Higgs CCEC Significance vs. $d R^{\gamma \gamma}(\eta, \phi)$ after the $p_{T}^{\gamma \gamma}$ cut is applied.

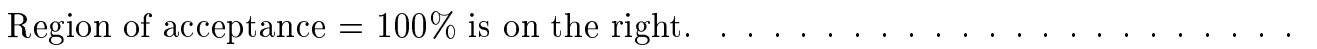

5.48 Topcolor Higgs CCCC Significance vs. $d R^{\gamma \gamma}(\eta, \phi)$ after the $p_{T}^{\gamma \gamma}$ cut is applied. Region of acceptance $=100 \%$ is on the right. $\ldots \ldots \ldots \ldots$

5.49 Topcolor Higgs CCEC Significance vs. $d R^{\gamma \gamma}(\eta, \phi)$ after the $p_{T}^{\gamma \gamma}$ cut is applied. Region of acceptance $=100 \%$ is on the right.

5.50 An example of correlations of $p_{T}^{\gamma \gamma}$ with other kinematic variables for $\mathrm{M}=120 \mathrm{GeV}$ Fermiophobic Higgs (CCCC) Top: $d R^{\gamma \gamma}(\eta, \phi)$ (vertical) vs. $p_{T}^{\gamma \gamma}$ (horizontal). Middle: $\Delta \phi$ (vertical) vs. $p_{T}^{\gamma \gamma}$ (horizontal). Bottom: $(E 1-E 2) /(E 1+E 2)$ (vertical) vs. $p_{T}^{\gamma \gamma}$ (horizontal).

5.51 Fermiophobic Higgs CCCC $|\Delta \eta(\gamma \gamma)|$ after the $p_{T}^{\gamma \gamma}$ cut. Dashed line - signal, solid line

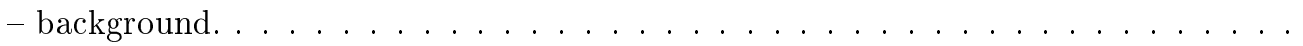

5.52 Fermiophobic Higgs CCEC $|\Delta \eta(\gamma \gamma)|$ after the $p_{T}^{\gamma \gamma}$ cut. Dashed line - signal, solid line - background.

5.53 Topcolor Higgs CCCC $|\Delta \eta(\gamma \gamma)|$ after the $p_{T}^{\gamma \gamma}$ cut. Dashed line - signal, solid line background.

5.54 Topcolor Higgs CCEC $|\Delta \eta(\gamma \gamma)|$ after the $p_{T}^{\gamma \gamma}$ cut. Dashed line - signal, solid line -

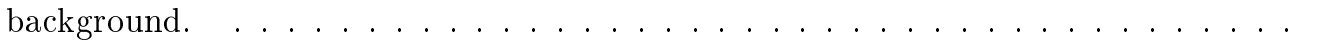

5.55 Helicity angle: angle between the direction of a photon momentum in the $\gamma \gamma$ rest frame and the diphoton momentum in the lab frame. $\ldots \ldots \ldots \ldots$ 
$5.56\left|\cos \theta^{*}\right|$ generator-level distribution for $90 \mathrm{GeV}$ Higgs. Top - all events, bottom after $p_{T}>25 \mathrm{GeV}$ is required for both photons. . . . . . . . . . . 143

5.57 Fermiophobic Higgs CCCC $(E 1-E 2) /(E 1+E 2)$ vs. $p_{T}^{\gamma \gamma}$. Small dots: signal, large dots: background. . . . . . . . . . . . . . . . . . . . . . . . . 144

5.58 Fermiophobic Higgs CCEC $(E 1-E 2) /(E 1+E 2)$ vs. $p_{T}^{\gamma \gamma}$. Small dots: signal, large dots: background. . . . . . . . . . . . . . . . . . . . . . . 145

5.59 Topcolor Higgs CCCC $(E 1-E 2) /(E 1+E 2)$ vs. $p_{T}^{\gamma \gamma}$. Small dots: signal, large dots: background. . . . . . . . . . . . . . . . . . . . . 146

5.60 Topcolor Higgs CCEC $(E 1-E 2) /(E 1+E 2)$ vs. $p_{T}^{\gamma \gamma}$. Small dots: signal, large dots: background. . . . . . . . . . . . . . . . . . . . . . 147

5.61 Fermiophobic Higgs CCCC $\left|\cos \theta^{*}\right|$ vs. $p_{T}^{\gamma \gamma}$. Small dots: signal, large dots: background.148 5.62 Fermiophobic Higgs CCEC $\left|\cos \theta^{*}\right|$ vs. $p_{T}^{\gamma \gamma}$. Small dots: signal, large dots: background. . . . . . . . . . . . . . . . . . . . . . . . . . . 149

5.63 Topcolor Higgs CCCC $\left|\cos \theta^{*}\right|$ vs. $p_{T}^{\gamma \gamma}$. Small dots: signal, large dots: background. 150

5.64 Topcolor Higgs CCEC $\left|\cos \theta^{*}\right|$ vs. $p_{T}^{\gamma \gamma}$. Small dots: signal, large dots: background.

5.65 Functional description of a node within a neural network is shown in the top diagram. The input $x_{i}$ is multiplied by the weight $w_{i j}$ and summed up. By applying a threshlod $\alpha_{i}$ and a transfer function $g(x)$ the output is computed. In the bottom diagram one can see a general neural net with input, hidden and output nodes. . . . . . . . . . 153

5.66 Significance as a function of the NN output cut for Fermiophobic Higgs (CCCC). Solid line: $p_{T}^{\gamma \gamma}, \cos \theta^{*},(E 1-E 2) /(E 1+E 2)$ input variable configuration; dahsed line: $p_{T}^{\gamma \gamma}, \cos \theta^{*}$; dotted line: $p_{T}^{\gamma \gamma},(E 1-E 2) /(E 1+E 2)$. One hidden layer with one hidden node was used. Solid straight line shows the significance for optimal $p_{T}^{\gamma \gamma}$

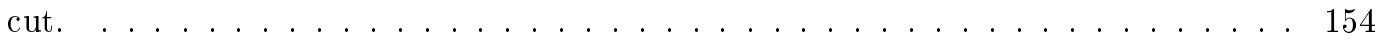

5.67 Neural Network output for signal and background for the case of $\mathrm{M}=60 \mathrm{GeV}$ Fermiophobic Higgs (CCCC). Filled histogram: signal; Open histogram: background. . . . 155 
5.68 Significance as a function of Neural Network output cut for different number of hidden nodes for Fermiophobic Higgs (CCCC). Solid line: 1 hidden node; dashed line: 3 hidden nodes; dotted line : 5 hidden nodes. Input variables are $p_{T}^{\gamma \gamma}$ and $\cos \theta^{*}$. Top plot corresponds to $\mathrm{M}=60 \mathrm{GeV}$, the bottom shows $\mathrm{M}=120 \mathrm{GeV}$ case. . . . . . . . 156

5.69 Contours of constant NN output in the $p_{T}^{\gamma \gamma}-\left|\cos \theta^{*}\right|$ plane for $\mathrm{M}=60 \mathrm{GeV}$ Fermiophobic Higgs signal $(\mathrm{CCCC}) . \ldots \ldots \ldots \ldots \ldots \ldots \ldots \ldots$

5.70 Contours of constant NN output in the $p_{T}^{\gamma \gamma}-\left|\cos \theta^{*}\right|$ plane for the CCCC background in the window centered at $\mathrm{M}=60 \mathrm{GeV} . \quad \ldots \ldots \ldots \ldots \ldots \ldots$. . . . . . . . . 157

6.1 Improving the limit via combining likelihoods (example for $\mathrm{M}=100 \mathrm{GeV}$ Topcolor Higgs). Top plot: likelihood function and derived limit for the CCCC subsample. The peak is due to fluctuation of 8 observed events above the expected background of $4.9 \pm 0.5$. Middle plot: likelihood function and derived limit for the CCEC subsample (Luminosity error - the dominant systematics - is set to zero), 3 observed events are below predicted background of $5.0 \pm 0.4$; such situation results in the absence of a peak in the likelihood shape; Bottom plot: combined CCCC-CCEC likelihood function and combined limit. The limit improves as a result of combining the two

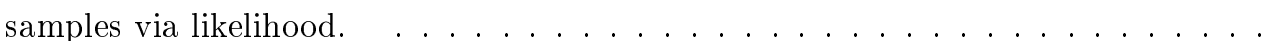

6.2 Example of worsening of the limit after combining the likelihoods $(\mathrm{M}=60 \mathrm{GeV}$ Fermiophobic Higgs). Normalization factors were changed for plotting purposes in order that different functions can be overlaid for visual comparison. Left curve: likelihood function and derived limit for the CCCC subsample. Right curve: likelihood function and derived limit for the CCEC subsample (Luminosity error - dominant systematics - is set to zero). Both CCCC and CCEC likelihoods have peaks since number of observed events fluctuates above the background in both cases. The broad shape of the latter corresponds to low acceptance in comparison with the former. Middle curve: combined CCCC-CCEC likelihood function and derived limit. The limit shifts slightly to

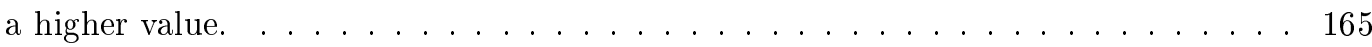


6.3 95\% CL cross section limits and NLO theoretical cross sections. Theoretical cross sections are shown with a line; points are experimental limits. Top plot: Fermiophobic Higgs scenario. Bottom plot: Topcolor Higgs scenario. . . . . . . . . . . . . 166

A.1 di-EM mass distributions for Tracking Efficiency measurement for CCCC events. Top plot shows mass spectrum and the fit for the whole sample, middle plot - events with exactly one track match, bottom plot - both EM objects are matched to tracks. . . 169

A.2 di-EM mass distributions for Tracking Efficiency measurement for CCEC events. Top plot shows mass spectrum and the fit for the whole sample, middle plot - events with exactly one track match, bottom plot - both EM objects are matched to a tracks. . 170

A.3 di-EM mass distributions for Tracking Efficiency measurement for ECEC events. Top plot shows mass spectrum and the fit for the whole sample, middle plot - events with exactly one track match, bottom plot - both EM objects are matched to tracks. . . 171 


\section{Chapter 1}

\section{Introduction}

Hope the voyage is a long one,

full of adventure, full of discovery.

Laistrygonians, Cyclops, angry Poseidon-

you won't encounter them

unless you bring them along inside your soul.

May there be many summer mornings when,

with what pleasure, what joy,

you enter harbours seen for the first time;

may you stop at Phoenician trading stations

to buy fine things,

and may you visit many Egyptian cities

to gather stores of knowledge from their scholars

Constantine Cavafy, "Ithaka"

Different stages in the development of Physics are closely connected with the distance scale between the objects under study. Not only certain effects could become stronger or weaker depending on the distance, e.g. the force of gravitational interaction, as described by the inverse square law, 
but also, as we get to smaller distances, qualitatively new phenomena emerge. This requires a new formalism to describe them and often even a change in the paradigm of thinking. For example, the laws of classical mechanics and electrodynamics can predict with accuracy the motion of the two-body system and the radiation from its charged constituents. However, same ideas and rules would no longer apply to the behavior of even a simplest small object of a similar structure the hydrogen atom. At this point, Quantum Mechanics came early last century. Along with the quantitative explanation of the atomic spectra it brought the probabilistic ideas, radically different from the deterministic classical description. As promising as this theory was though, it alone was not sufficient to describe the subatomic phenomena; it had to be combined with special relativity and applied to a system with an infinite number of degrees of freedom (a field) in order to explain interactions of elementary particles.

This resulted in the Standard Model (SM) of electroweak and strong interactions developed in the 60's by Glashow, Salam, and Weinberg [3], which serves as our current model of the physical world down to the distances $\sim 10^{-15} \mathrm{~cm}$. The Standard Model has an overwhelming experimental confirmation of its predictions. To name just a few of these experimental tests in 1983, $\mathrm{W}$ and $\mathrm{Z}$ bosons were discovered by the UA1 and UA2 collaborations $[4,5,6]$ at the CERN $p \bar{p}$-collider and in 1995 the top quark was discovered by the $\mathrm{CDF}$ and $\mathrm{D} \varnothing$ collaborations at the Tevatron $p \bar{p}$-collider at Fermilab [7, 8].

It was in the light of these developments of the last century that mass (known as a measure of inertia), the quantity that seemed well understood since Newton's time, started to raise very intriguing observations and questions to many of which we still do not have answers. Double-Slit Quantum Mechanical Experiment showed that electron, a particle with well-defined and measured mass, also behaved as a wave. Albert Einstein came up with the famous $E=m c^{2}$ statement of the equivalence of mass and energy. Bargman and Wigner [9] showed that mass together with spin are two fundamental quantities by which all relativistic wave equations (and therefore elementary particles) can be classified. Trying to reconcile special relativity with gravity, the interaction between two objects that have mass, Einstein came to a profound conclusion about the significance of mass: 
the distribution of mass in the Universe defines the geometry of space-time [10]. This sets gravity apart from other known interactions, all of which occur in space-time without affecting it.

Finally, there is a fundamental question of the origin of mass. The Standard Model suggests that mass can not be viewed as an intrinsic property of a particle, but rather comes from some interaction. A way to introduce mass in the SM is to add an extra field - Higgs field [11], interacting with which massless particles acquire mass. An experimental verification of this hypothesis would be a discovery of an excitation of the Higgs field - the Higgs boson.

Work described in this dissertation is a contribution to the Higgs boson search performed at the DØ detector in Run II of the Tevatron. The dissertation contains five additional chapters. Chapter 2 (Theory and Phenomenology) gives an overview of the SM and the Higgs mechanism, discusses Higgs production and decay modes in the SM and in some of its extensions, with the focus on the $\gamma \gamma$ decay mode. Chapter 3 (Fermilab and the DØ Detector) provides a short description of the Fermilab Tevatron proton-antiproton collider and the $\mathrm{D} \varnothing$ detector, which was used to collect data for this analysis. Chapter 4 (Photon Identification) describes the features of electromagnetic and hadronic shower development and the techniques used to identify photons in the detector. Measurements of photon efficiency and misidentification rate are discussed. Chapter 5 (Analysis) explains specifics of the event selection, estimation of SM backgrounds, as well as signal efficiencies and acceptances. Chapter 6 (Higgs Cross Section Limits) contains the cross section limits obtained in the analysis. 


\section{Chapter 2}

\section{Theory and Phenomenology}

\subsection{Standard Model}

I know the composition of the biosphere,

the structure of acids,

all that is found in nature, I accept as a given.

I am from the twentieth century, and behold,

astonished, I see just first-creation.

Lina Kostenko, "All that will be, already is or has happened on earth..."

\subsubsection{Particles of the Standard Model}

The Standard Model is a relativistic quantum field theory based on the principle of least action and local gauge invariance $[12,13]$. Particles are described as field excitations above the ground state (vacuum). There are two types of elementary particles in the Standard Model: spin 1/2 fermions and spin 1 and 0 bosons. All fermions are massive ${ }^{1}$ and are called matter particles of building blocks of matter. Some of them, namely up-quark, down-quark, and electron, can form stable bound states

\footnotetext{
${ }^{1}$ The neutrino sector could be an exception. One of the neutrinos may be massless
} 
(proton, neutron, atoms, molecules) out of which the stars, the planets, and the Natural world on Earth is made. Interactions between matter particles (scattering off one another, production and decay, bound-state-forming attraction) happen via exchange of spin 1 bosons, which are called mediators or force carriers. There are three types of interactions - electromagnetic, weak, and strong; their carriers being photon, $\mathrm{W} / \mathrm{Z}$ bosons, and gluon, respectively. Photon and gluon are massless while $\mathrm{W}$ and $\mathrm{Z}$ bosons have mass. At the moment the fourth, gravitational interaction is not a part of the SM.

\subsubsection{Local Gauge Invariance}

The very existence of force carriers and interactions follows from the requirement that the Lagrangian of matter fields is invariant under the local gauge transformations. Gauge transformations form mathematical groups. Choosing a particular gauge group, leads to a specific type of interaction. This can be illustrated by an example of electromagnetic interaction [15]. The fermion field part of the Lagrangian

$$
\mathcal{L}=i \hbar c \bar{\psi} \gamma^{\mu} \partial_{\mu} \psi-m c^{2} \bar{\psi} \psi
$$

is not invariant under the $\mathrm{U}(1)$ gauge transformation

$$
\psi \rightarrow e^{-i q \lambda(x) / \hbar c} \psi
$$

as an extra term is picked up from the derivative of $\lambda(x)$ :

$$
\mathcal{L} \rightarrow \mathcal{L}+\left(q \bar{\psi} \gamma^{\mu} \psi\right) \partial_{\mu} \lambda(x)
$$

However, if we introduce an extra field $A_{\mu}$ that transforms as $A_{\mu} \rightarrow A_{\mu}+\partial_{\mu} \lambda(x)$ the Lagrangian (2.1) becomes:

$$
\mathcal{L}=i \hbar c \bar{\psi} \gamma^{\mu} \partial_{\mu} \psi-m c^{2} \bar{\psi} \psi-\left(q \bar{\psi} \gamma^{\mu} \psi\right) A_{\mu}
$$


and the gauge invariance is preserved. The extra term describes the interaction between fermions and a massles vector gauge boson (photon).

Other interactions can be derived in a similar way by studying more general gauge transformations [16]. If we consider the $\mathrm{SU}(2)$ transformation of the fermion doublet, the local gauge invariance requires three massless vector bosons - carriers of weak force.

\subsubsection{Electroweak Symmetry Breaking}

In the Glashow-Salam-Weinberg model, the electromagnetic and weak interactions are combined under the $\mathrm{SU}(2) \mathrm{xU}(1)$ group [22]. We denote $\mathrm{SU}(2)$ and $\mathrm{U}(1)$ gauge fields as $W_{\mu}^{i}, \mathrm{i}=1,2,3$ and $B_{\mu}$, respectively, and the corresponding coupling constants as $g$ and $g^{\prime}$. The left-handed fermion fields:

$$
\psi_{i L}=\left(\begin{array}{c}
\nu_{i} \\
l_{i}^{-}
\end{array}\right)_{L} \text { and }\left(\begin{array}{c}
u_{i} \\
d_{i}^{\prime}
\end{array}\right)_{L}
$$

transform as doublets under $\mathrm{SU}(2)$, where $d_{i}^{\prime} \equiv \sum_{j}^{3} V_{i j} d_{j}$, and V is the Cabibbo-Kobayashi-Maskawa mixing matrix. The right-handed fields are the SU(2) singlets. Such theory is incomplete as all of the four gauge bosons are predicted to be massless, which has been ruled out by the discovery of massive $W^{ \pm}$and $Z^{0}$ weak bosons. The massiveness of weak gauge bosons can be explained by incorporating into the theory an extra spin 0 complex doublet, the Higgs field [11]:

$$
\phi \equiv\left(\begin{array}{c}
\phi^{+} \\
\phi^{0}
\end{array}\right)
$$

This field is neither matter field nor gauge-mediator field. The Lagrangian of the Higgs field (using $c \equiv 1, \hbar \equiv 1)$

$$
\mathcal{L}=\left(\partial_{\mu} \phi\right)^{\dagger}\left(\partial^{\mu} \phi\right)-\mu^{2} \phi^{\dagger} \phi-\lambda\left(\phi^{\dagger} \phi\right)^{2}
$$

respects local gauge invariance and so does the complete set of its ground states. However, when a particular ground state is chosen and the fields are rewritten in terms of $\mathrm{v}$ and $\mathrm{H}, \mathrm{v}$ being the 
vacuum expectation value (vev) of the Higgs field and $\mathrm{H}$ the real field with zero vev [16], the Higgs field becomes

$$
\phi \equiv\left(\begin{array}{c}
\phi^{+} \\
\phi^{0}
\end{array}\right) \rightarrow \frac{1}{\sqrt{2}}\left(\begin{array}{c}
0 \\
v+H
\end{array}\right)
$$

and breaks the $\mathrm{SU}(2) \mathrm{xU}(1)$ symmetry. This symmetry breaking leads to and extra three degrees of freedom that correspond to the zero-energy excitations along the ground state surface of the unbroken theory. These extra degrees of freedom appear as additional (longitudinal) polarizations of the original $W_{\mu}^{i}$ bosons, i.e. weak gauge bosons acquire mass. Higgs mechanism not only explains the mass of $W^{ \pm}$and $Z^{0}$ bosons, but also the fermion masses and predicts a weakly interacting spin-0 massive particle - the Higgs boson. Parameters v, $\mu$ and $\lambda$ of the Higgs field are related via $v^{2}=\frac{-\mu 2}{\lambda}$ relation [14]. The parameter $\mathrm{v}$ can be expressed in terms of the Fermi coupling constant (which was determined in the muon lifetime measurement) [16]:

$$
v=\left(\sqrt{2} G_{F}\right)^{-1 / 2}=246 \mathrm{GeV}
$$

This leaves a single independent parameter, which can not be determined indirectly without having experimental information about the Higgs boson. This unknown parameter can be rewritten as the Higgs boson mass $m_{H}=\sqrt{-2 \mu^{2}}$.

\subsubsection{Standard Model Lagrangian}

After spontaneous symmetry breaking, the electroweak Lagrangian can be written as:

$$
\mathcal{L}=\mathcal{L}_{\mathcal{F}}+\mathcal{L}_{\phi}+\mathcal{L}_{\mathcal{W}}
$$

where $\mathcal{L}_{\mathcal{F}}$ is the fermion field Lagrangian, $\mathcal{L}_{\phi}$ describes the Higgs part (kinetic and mass term, Higgs self-interactions, and Higgs interactions with the gauge bosons), and $\mathcal{L}_{\mathcal{W}}$ contains gauge boson mass terms, as well as the gauge boson interactions. 
The fermion lagrangian $\mathcal{L}_{\mathcal{F}}$ has the following form

$$
\begin{aligned}
\mathcal{L}_{\mathcal{F}} & =\sum_{i} \bar{\psi}_{i}\left(\gamma^{\mu} \partial_{\mu}-m_{i}\right) \psi_{i} \\
& -\frac{g}{2 M_{W}} \sum_{i} m_{i} \bar{\psi}_{i} \psi_{i} H \\
& -\frac{g}{2 \sqrt{2}} \sum_{i} \bar{\psi}_{i} \gamma^{\mu}\left(1-\gamma^{5}\right)\left(T^{+} W_{\mu}^{+}+T^{-} W_{\mu}^{-}\right) \psi_{i} \\
& -e \sum_{i} q_{i} \bar{\psi}_{i} \gamma^{\mu} \psi_{i} A_{\mu} \\
& -\frac{g}{2 \cos \theta_{W}} \sum_{i} \bar{\psi}_{i} \gamma^{\mu}\left(g_{i}^{V}-g_{i}^{A} \gamma^{5}\right) \psi_{i} Z_{\mu}
\end{aligned}
$$

where $\theta_{W} \equiv \tan ^{-1}\left(g^{\prime} / g\right)$ is the weak angle; $e=g \sin \theta_{W}$ is the electric charge of the positron, and $A, W^{ \pm}, Z$ are the new gauge boson fields: $A \equiv B \cos \theta_{W}+W^{3} \sin \theta_{W}$ is the (massless photon field) $W^{ \pm} \equiv\left(W^{1} \mp i W^{2}\right) \sqrt{2}$ and $Z \equiv-B \sin \theta_{W}+W^{3} \cos \theta_{W}$ are the massive charged and neutral weak boson fields, respectively. (Here $T^{+}$and $T^{-}$are the weak isospin rasing and lowering operators, $g_{i}^{V}$ and $g_{i}^{A}$ are the vector and axial vector couplings.)

First term in (2.8) is the fermion field lagrangian (2.1) in the absence of any other fields (Dirac Lagrangian); the second term describes Higgs-fermion interaction; the third term represents the charged-current weak interaction. For example, the coupling of a $W$ to an electron and a neutrino is

$$
-\frac{e}{2 \sqrt{2} \sin \theta_{W}}\left[W_{\mu}^{-} \bar{e}_{L} \gamma^{\mu}\left(1-\gamma^{5}\right) \nu_{L}+W_{\mu}^{+} \bar{\nu}_{L} \gamma^{\mu}\left(1-\gamma^{5}\right) e_{L}\right]
$$

The fourth term in (2.8) describes electromagnetic interaction (QED), and the last term represents the weak neutral-current interaction.

The $\mathcal{L}_{\phi}$ (Higgs) term in the electroweak Lagrangian has the following form [6]:

$$
\begin{aligned}
\mathcal{L}_{\phi} & =\frac{1}{2}(\partial H)^{2}-\frac{1}{2} m_{H}^{2} H^{2} \\
& -\frac{1}{2} \frac{m_{H}^{2}}{v} H^{3} \\
& -\frac{1}{8} \frac{m_{H}^{2}}{v^{2}} H^{4} \\
& +\left(\frac{1}{4} W_{\mu}^{+} W^{-\mu}+\frac{1}{8} \frac{g^{2}}{\sin ^{2} \theta_{W}} Z_{\mu} Z^{\mu}\right)\left(H^{2}+2 v H\right)
\end{aligned}
$$


the first line (kinetic and mass terms) is the scalar field (Klein-Gordon) Lagrangian; the second and third lines describe cubic and quartic Higgs self-couplings, respectively; the fourth line represents $W W H, Z Z H, W W H H$, and $Z Z H H$ interactions.

Finally, the $\mathcal{L}_{\mathcal{W}}$ part of the electroweak Lagrangian has the following form [6]:

$$
\begin{aligned}
\mathcal{L}_{\mathcal{W}} & =m_{W}^{2} W_{\mu}^{+} W^{-\mu}+\frac{1}{2} m_{Z}^{2} Z_{\mu} Z^{\mu} \\
& -\frac{1}{4} W_{\mu \nu} W_{i}^{\mu \nu}-\frac{1}{4} B_{\mu \nu} B^{\mu \nu}
\end{aligned}
$$

with

$$
W_{\mu \nu}=\partial_{\mu} W_{i \nu}-\partial_{\nu} W_{i \mu}-g f_{i j k} W_{j \mu} W_{k \nu}
$$

where $f_{i j k}$ are the structure constants of the $\mathrm{SU}(2)$ weak isospin group: $\left[T_{i}, T_{j}\right]=i f_{i j k} T_{k}$. In the standard, $2 \times 2$ unitary representation of the $\mathrm{SU}(2)$ group $T_{i}=\tau_{i} / 2\left(\tau_{i}\right.$ are the Pauli matrices) and $f_{i j k}=\epsilon_{i j k}$ (totally antisymmetric tensor).

The first line in $L_{\mathcal{W}}$ corresponds to the $\mathrm{W}$ and $\mathrm{Z}$ mass terms; the second line is responsible for cubic $(W W Z, W W \gamma)$ and quartic $(W W W W, W W Z Z, W W Z \gamma, W W \gamma \gamma)$ self-interactions of the gauge fields.

The remaining component of the Standard Model is Quantum Chromodynamics (QCD) [22]. QCD is the $\mathrm{SU}(3)$ gauge field theory, which describes strong interactions of colored quarks and gluons. A quark of a specific flavor (such as a charm quark) comes in three colors; gluons come in eight colors; hadrons are color-singlet combinations of quarks and anti-quarks, bound with gluons. The Lagrangian describing the interactions of quarks and gluons is (up to gauge-fixing terms):

$$
\begin{aligned}
\mathcal{L}_{\mathcal{Q C D}} & =-\frac{1}{4} F_{\mu \nu}^{(a)} F^{(a) \mu \nu} \\
& +i \sum_{q} \bar{\psi}_{q}^{i} \gamma^{\mu}\left(D_{\mu}\right)_{i j} \psi_{q}^{j} \\
& -\sum_{q} m_{q} \bar{\psi}_{q}^{i} \psi_{q i},
\end{aligned}
$$


with

$$
\begin{aligned}
F_{\mu \nu}^{(a)} & =\partial_{\mu} A_{\nu}^{a}-\partial_{\nu} A_{\mu}^{a}-g_{s} f_{a b c} A_{\mu}^{b} A_{\nu}^{c} \\
\left(D_{\mu}\right)_{i j} & =\delta_{i j} \partial_{\mu}+i g_{s} \sum_{a} \frac{\lambda_{i, j}^{a}}{2} A_{\mu}^{a}
\end{aligned}
$$

where $g_{s}$ is the QCD coupling constant, $f_{a b c}$ are the structure constants of the $\mathrm{SU}(3)$ group, and $\lambda^{a}, a=1, \ldots, 8$ denote the $3 \times 3 \mathrm{SU}(3)$ generator matrices. The $\psi_{q}^{i}(x)$ are the 4-component Dirac spinors associated with each quark field of color $i=1,2,3$ and flavor $q$, and the $A_{\mu}^{a}(x)$ are the eight Yang-Mills (gluon) fields. Lagrangians (2.7) and (2.12) compose the full $S U(3) \times S U(2) \times U(1)$ Standard Model Lagrangian.

\subsubsection{Cross Sections and Decay Widths}

Observable quantities such as cross sections and decay widths can be calculated using coupling constant perturbative expansion of corresponding Lagrangians. Individual terms in the expansion can be represented by Feynman diagrams and evaluated using Feynman rules that associate corresponding factors with incoming, virtual, outgoing particles and vertices in a diagram. The strength of a particular coupling constant depends on the amount of energy transferred in the interaction (running of the coupling constants). Electromagnetic coupling increases with energy whereas strong coupling decreases ("asymptotic freedom"). Therefore for electromagnetic processes perturbation theory is applicable at low energies while QCD perturbative calculations can be performed only at high energies. Soft QCD requires non-perturbative approach. 


\title{
2.2 Beyond the Standard Model
}

This section briefly describes some of the ideas that motivate Physics beyond the SM and outlines its major areas. It is not meant to be a comprehensive summary of the subject.

\subsubsection{Why the Standard Model Needs to be Extended}

\author{
These noble thoughts beguiled us yesterday; \\ We savored them like choicest vintage wines. \\ But now they sour, meanings seep away. \\ from Joseph Knecht's \\ summer homework assignments
}

While the Standard Model provides a formalism for electroweak symmetry breaking and has been verified experimentally there are indications that it is only a low-energy approximation of a more general physical theory. One of the arguments against the Standard Model as an ultimate theory is purely mathematical and arises when radiative corrections to the Higgs boson mass are computed. At one loop, the quartic self-interactions of the Higgs Boson ( $\lambda^{4}$ term in the Higgs Lagrangian (2.10), where $\left.\lambda \sim \frac{m_{H}^{2}}{v^{2}}\right)$ generate a quadratically divergent contribution to the Higgs boson mass which must be cancelled by the mass counterterm, $\delta M_{h}^{2}[19]$

$$
M_{h}^{2} \sim M_{h 0}^{2}+\frac{\lambda}{4 \pi^{2}} \Lambda^{2}+\delta M_{h}^{2}
$$

The scale $\Lambda$ is the highest scale in the theory, which is of the order of the Planck scale, if the SM is the ultimate theory The mass of the Higgs boson must be less than around $800 \mathrm{GeV}$ to prevent unitarity $^{2}$ violation in the scattering of longitudinal gauge bosons. This leads to an unsatisfactory situation. The large quadratic contribution to the Higgs boson mass squared, of $\mathcal{O}\left(10^{18} \mathrm{GeV}\right)^{2}$, must

\footnotetext{
${ }^{2}$ Unitarity, or the conservation of probability, is imposed as a constraint in the construction of any quantum field theory $[12,13]$. This leads to the requirement that all production cross-sections must fall as $s^{-1}$ at energies above the mass of the produced particle. The scattering of the longitudinal gauge bosons violates this limit when the Higgs is too heavy [18].
} 
be cancelled by the counterterm $\delta M_{h}^{2}$ such that the result is roughly less than $(800 \mathrm{GeV})^{2}$. This requires a cancellation of one part to $\left(10^{16}\right)^{2}=10^{32}$. Formally this is possible but is regarded by most theorists as unacceptable fine tuning of parameters. Moreover, this cancellation must occur at every order of the perturbation theory, so the parameters need to be retuned. This is known as a fine tuning problem [12, 17].

Unexplained large difference between the electroweak and the Planck scale by itself is considered to be a serious shortcoming of the Standard Model (the hierarchy problem). Besides, the Standard Model does not explain the CP-violation, neutrino masses, or CKM matrix elements.

\subsubsection{Supersymmetry}

Supersymmetry is a symmetry that connects particles of different spin, with all other characteristics being the same [19]. The particles are combined into a superfield, which contains fields differing by one-half unit of spin [20]. Suppose we reconsider the one loop contributions to the Higgs boson mass in a theory which contains both massive scalars, $\phi$, and fermions, $\psi$ in addition to the Higgs field $h$. Then the Lagrangian is given by:

$$
\mathcal{L} \sim g_{F} \bar{\psi} \psi h-g_{S}^{2} h^{2} \phi^{2}
$$

If we again calculate the one-loop contribution to $M_{h}^{2}$ we find

$$
M_{h}^{2} \sim M_{h 0}^{2}+\frac{g_{F}^{2}}{4 \pi^{2}}\left(\Lambda^{2}+m_{F}^{2}\right)-\frac{g_{S}^{2}}{4 \pi^{2}}\left(\Lambda^{2}+m_{S}^{2}\right)+\text { logarithmic divergences }+ \text { uninteresting terms }
$$

The relative minus sign between the fermion and scalar contributions to the Higgs boson masssquared is the well-known result of Fermi statistics. We see that if $g_{F}=g_{S}$ the terms which grow as $\Lambda^{2}$ cancel and we are left with a well-behaved contribution to the Higgs boson mass, as long as the fermion and scalar masses are not too different.

$$
M_{h}^{2} \sim M_{h 0}^{2}+\frac{g_{F}^{2}}{4 \pi^{2}}\left(m_{F}^{2}-m_{S}^{2}\right)
$$


The equality of fermion and boson masses is a characteristic of unbroken supersymmetric theories. However supersymmetry must be a broken symmetry. For example, there is no scalar particle with the mass and quantum numbers of the electron. The mechanism of the supersymmetry breaking is not well understood. There exist several ways of introducing the supersymmetry breaking:

- In the Minimal Supersymmetric Model (MSSM) the supersymmetry breaking is simply introduced explicitly;

- Gauge Mediated theories (GMSB) introduce new gauge field that couples to the source of SUSY-breaking with ordinary and SUSY matter;

- In Gravity Mediated theories (SUGRA) SUSY breaking is propagated through gravitational interaction;

- SUSY breaking is mediated by gauginos (supersymmetric partners of gauge bosons).

These theories predict different spectra and phenomenology of supersymmetric particles.

The SUSY Higgs sector consists of more than one Higgs boson. For example, in the MSSM model, two massive complex scalar Higgs doublets are introduced, which after $S U(2) \times U(1)$ symmetry breaking, with the vacuum expectation values $v_{1}$ and $v_{2}$ respectively (their ratio $\tan \beta=v_{1} / v_{2}$ is a parameter of a SUSY model) result in five physical Higgs bosons:

- charged pair $H^{ \pm}$;

- two neutral CP-even scalars $h^{0}$ and $H^{0}\left(m_{H^{0}}>m_{h^{0}}\right)$;

- neutral CP-odd scalar $A^{0}$.

\subsubsection{Strong Dynamics}

In the Standard Model and in SUSY models electroweak symmetry breaking is introduced with the help of fundamental Higgs boson(s) [21]. An alternative view puts the origin of the symmetry breaking in a different sector of the theory, one with new fundamental fermions that have new gauge interactions. In this approach elementary scalar bosons are completely absent. Scalar and pseudoscalar bosons that are built of the new fundamental fermions can exist (in analogy with the 
way pions arise in the QCD theory of quarks). Such theories include, e.g. Technicolor, Extended Technicolor, Topcolor, Topcolor-assisted Technicolor,

\subsubsection{Extra Dimensions}

The issue of large separation between the weak scale $\sim 10^{3} \mathrm{GeV}$ and the Planck scale $\sim 10^{19} \mathrm{GeV}$ (the traditional scale of gravity) mentioned in section 2.2.1 is known as the hierarchy problem. In addition to SUSY approach it also can be addressed by exploiting the geometry of space-time [22]. Specifically, recent theories involve the idea that the 3 -spatial dimensions in which we live could form a 3-dimensional 'membrane' embedded in a much larger extra dimensional space and that the hierarchy is generated by the geometry of the additional dimensions. Such ideas have led to extra dimensional theories which have verifiable consequences at the $\mathrm{TeV}$ scale. Our lack of knowledge of gravity at distances less than roughly a millimeter leads to the possibility that matter and non-gravitational forces are confined to our 3-dimensional subspace whereas gravity may propagate throughout higher-dimensional volume (bulk). In this case, the gauge forces are trapped within our 3-dimensional space, unaware of extra dimensions, and maintain their usual behaviour. Gravity, on the other hand, in this scheme is modified; its force would no longer follow the inversesquare law at distances smaller than the size of extra dimensions, as the gravitational equivalent of Gauss's Law mandates that the gravitational field spreads out into the full spatial volume.

There are three principal scenarios with predictions at the TeV scale, each of which has a distinct phenomenology :

- Large Extra Dimensions;

- $\mathrm{TeV}^{-1}$-size Extra Dimensions with SM Fields;

- Warped extra dimensions.

Higgs sector in the Extra-Dimensional theories depends on specific scenarios. Generally several physical Higgs states appear. 


\title{
2.3 Higgs Production and Decay
}

\author{
'How do you know?', \\ said the Mathematical Master, \\ 'you have never seen one' \\ 'Ah, but we have in our dreams', \\ answered the children. \\ Oscar Wilde, "The Happy Prince"
}

In this section we will focus on the production of the Higgs boson at the Tevatron $p \bar{p}$ collider. Also Higgs decay modes will be discussed with the emphasis on the $\gamma \gamma$ decays in the SM and some of its extensions.

\subsubsection{Higgs Production at the Tevatron}

Higgs-fermion interaction is given by the first term of equation (2.8) :

$$
\frac{g}{2 M_{W}} m_{f} \bar{\psi} \psi H
$$

where $m_{f}$ is the fermion mass. Since the coupling is proportional to the fermion mass, production of the Higgs via fermion fusion has a small cross-section as the quark constituents of a proton and antiproton have low mass $(\sim \mathrm{MeV})$. Gluon fusion is forbidden on the tree-level since the Higgs is colorless. Dominant production mechanism is the gluon fusion with a quark loop. The Feynman diagram for this process is shown in Figure 2.1.

The cross-section for a process given by such loop diagram is proportional to $m^{2}$ ( $m$ is mass of the particle in the loop). Therefore the dominant contribution to gluon fusion is expected from the top-quark loop. Also Higgs boson can appear in processes in which $\mathrm{W}$ or $\mathrm{Z}$ bosons are produced. These are vector boson associated production and vector boson fusion processes shown in Figure 2.2. Figure 2.3 shows Higgs production cross-section as a function of Higgs mass for $2 \mathrm{TeV}$ center-of-mass 


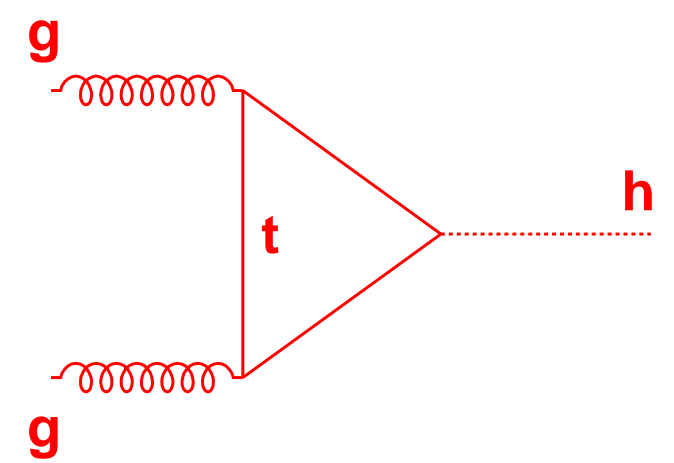

Figure 2.1: Higgs production in the $p \bar{p}$ collision via gluon fusion.

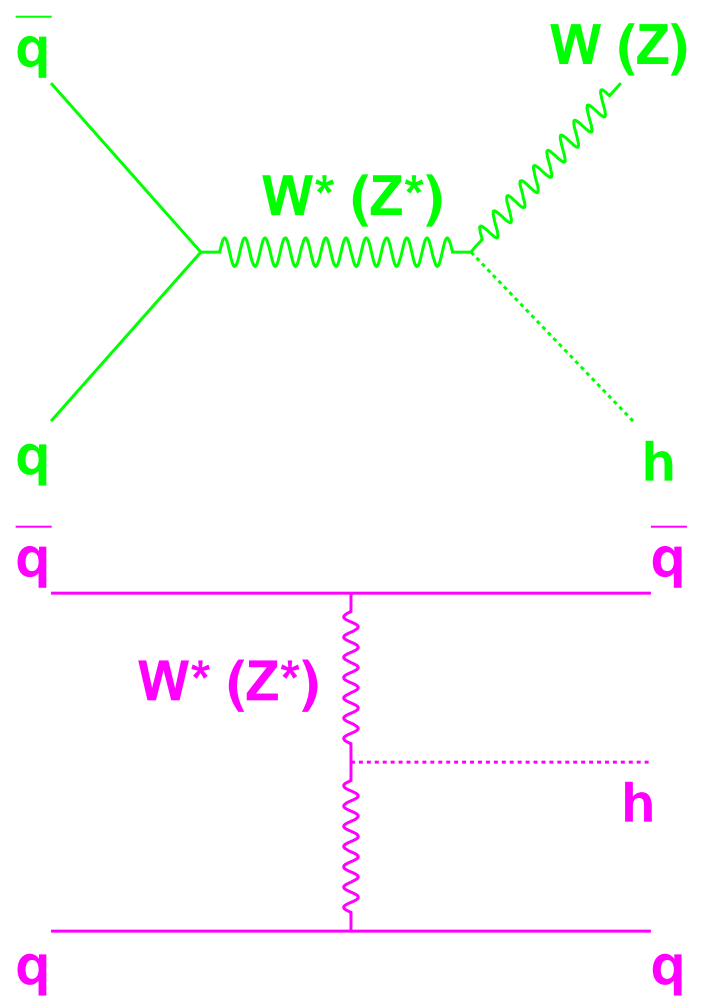

Figure 2.2: Higgs production in the $p \bar{p}$ collision via $\mathrm{W} / \mathrm{Z}$ associated production (top diagram) and in $\mathrm{W} / \mathrm{Z}$ fusion (bottom diagram). 
energy.

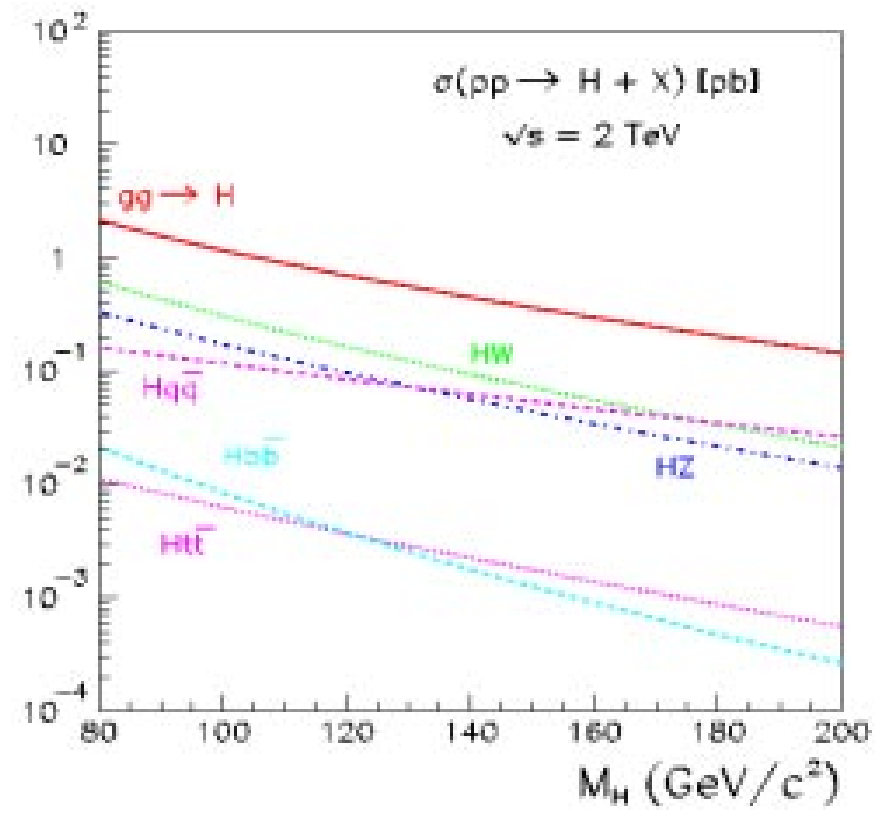

Figure 2.3: Higgs production cross section at the Tevatron as a function of Higgs mass [23, 24, 25].

\subsubsection{Higgs Decays}

Same considerations of Higgs couplings would apply to the discussion of Higgs decay modes. The tree-level decay to two gluons is forbidden and the one-loop gluon decay is allowed with the top-quark loop being dominant.

Decay branching fractions to fermions increase with the fermion mass; the dominant fermion mode in the $\sim 50-150 \mathrm{GeV}$ Higgs mass range being $b \bar{b}$ mode. At $\sim 100 \mathrm{GeV}, W W *$ decays start to be competitive with $b \bar{b}$, and above $2 M_{W}$ the $W W$ mode becomes dominant, followed by the $Z^{(*)} Z$. Figure 2.4 shows the SM branching fractions as a function of Higgs mass.

Being neutral, Higgs does not couple to photons at tree level. In the SM, the diphoton decays only happen via the top-quark or $W$ loop shown in Figure 2.5. Therefore, the diphoton decay branching fraction (not shown in Figure 2.4) is very small in the SM: $10^{-3}-10^{-4}$. However there are extensions of the SM that predict an enhanced decay rate into the $\gamma \gamma$ channel and simultaneous suppression of the $h \rightarrow b \bar{b}$ channel [26]. One example is the "bosophillic" or "fermiophobic" Higgs 


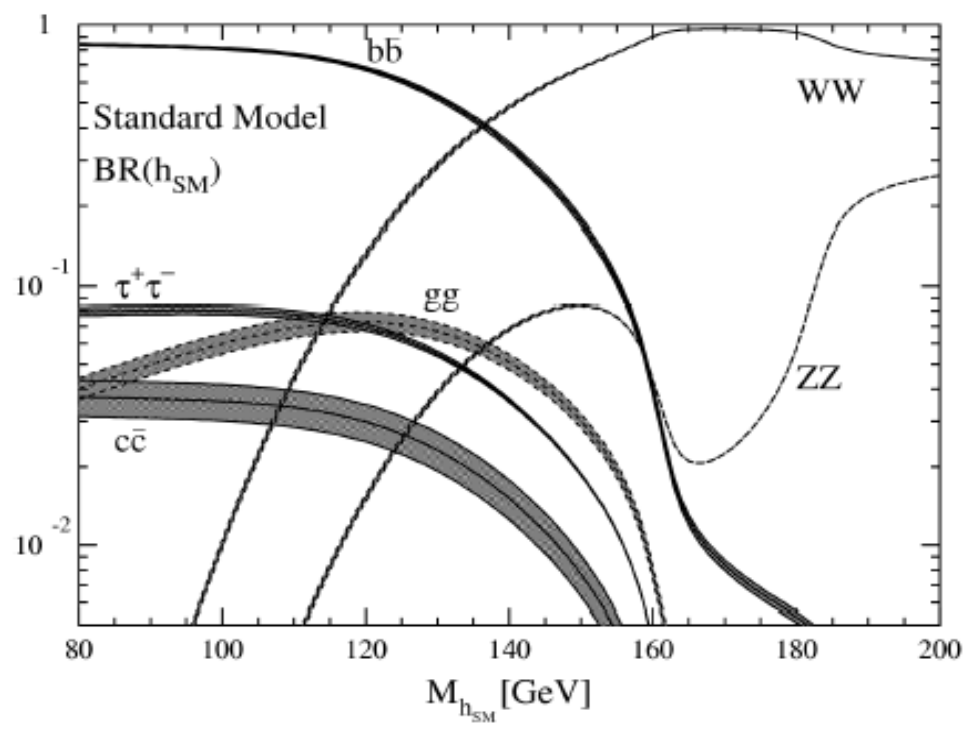

Figure 2.4: SM Higgs decay branching fractions. The $\gamma \gamma$ branching fraction $\left(\sim 10^{-3}-10^{-4}\right)$ is not shown.
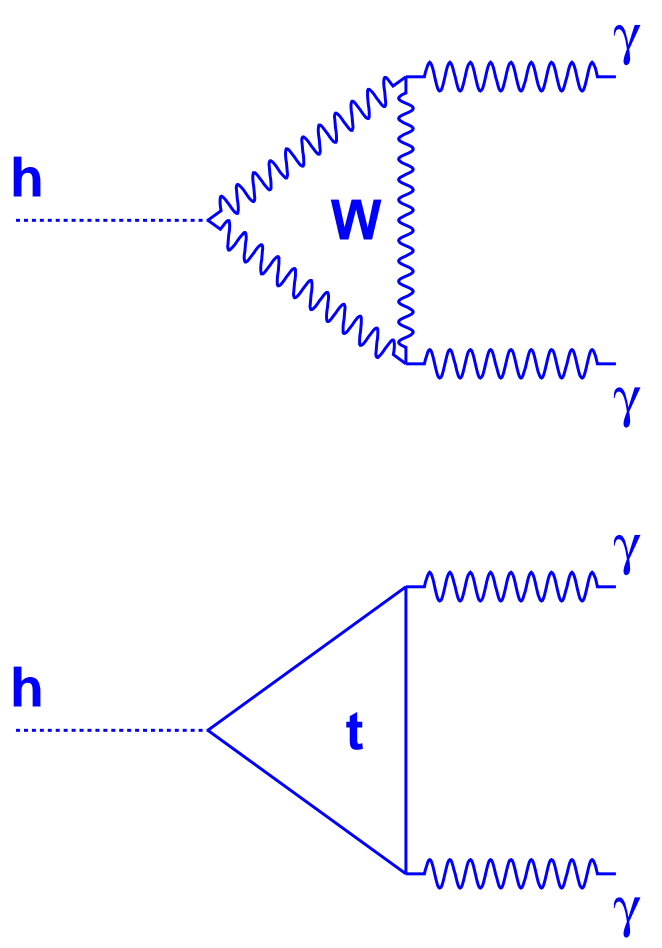

Figure 2.5: Diphoton Higgs decays. Top diagram: W-loop decay, bottom diagram: top-quark loop decay. 
[27] $h_{b h}^{0}$ that gives all (or nearly all) the mass to the vector bosons, but has no couplings to fermions. Another interesting departure from the SM Higgs phenomenology occurs when the light Higgs boson mass eigenstate of supersymmetry is the weak eigenstate $h_{u}^{0}[28,29,30,31]$. This scenario and its close variations naturally occur in theories with large $\tan \beta=\left\langle H_{u}\right\rangle /\left\langle H_{d}\right\rangle$, which are motivated by supersymmetric $\mathrm{SO}(10)$ grand unification [32]. Another example that has suppressed couplings to fermions is an electroweak Higgs boson $h_{e w}^{0}$ present in some top-quark condensate models [33]. In this approach, the top and bottom quarks are assumed to get their masses through a strongly coupled group that condenses top quark pairs [34], and all the remaining fermions and vector bosons get mass mainly through $\left\langle h_{e w}^{0}\right\rangle$. Figure 2.6 shows branching fractions into two photons for the SM Higgs and the three Higgs bosons mentioned above. Yet another variation is the Higgs in certain topcolor

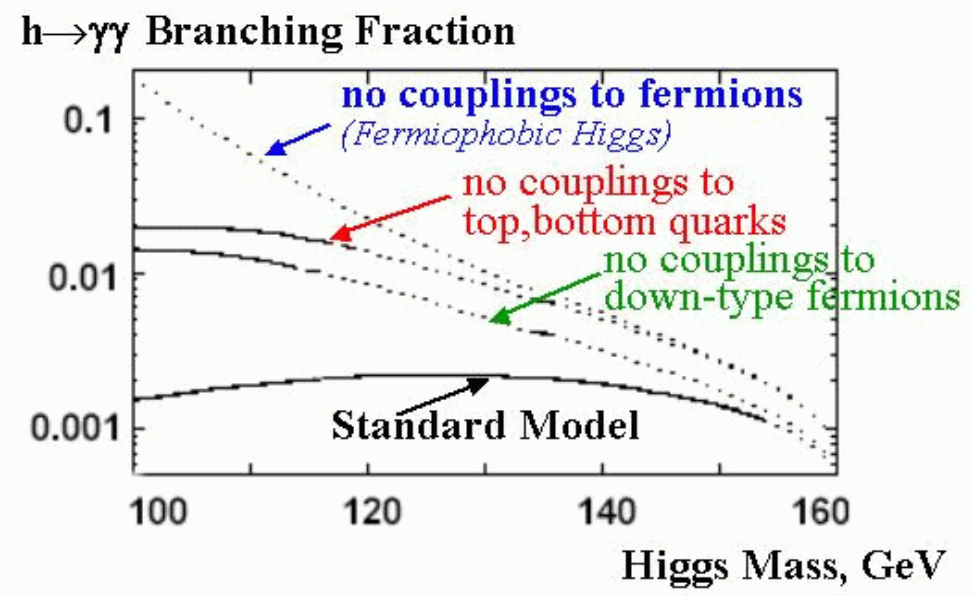

Figure 2.6: Examples of enhanced diphoton decays of the Higgs. Lines indicate (top to bottom): fermiophobic (bosonic) Higgs $h_{b h}^{0}$; electroweak Higgs $h_{e w}^{0}$ with zero couplings to the top and bottom quarks; Higgs that couples only to up-type fermions $h_{u}^{0}$; the Standard Model Higgs.

models which may couple to heavy quarks only $[35,36]$. Some even more exotic possibilities have been suggested in the context of large extra dimensions [37]. In addition, the $\gamma \gamma$ decay loop may be enhanced by a contribution from a heavy charged particle that may arise in other new physics scenarios.

To summarize this section, there are many theories that come from various theoretical frameworks that predict quantitatively different enhancement of the $h \rightarrow \gamma \gamma$ mode. In general, we should be prepared [26] to discover and study a Higgs boson with any branching fraction to two photons, from 
0 to 1 , since that is perhaps the most likely decay to be significantly altered by new physics. From the experimental point of view, $\gamma \gamma$ is a relatively clean final state, which makes the $h \rightarrow \gamma \gamma$ channel altogether promising and interesting research topic.

It is convenient to perform a search as a function of the branching fraction to two photons and reduce the list of possibilities to just two scenarios based on the dominant production mechanisms. These two scenarios are called Fermiophobic Higgs and Topcolor Higgs. A Fermiophobic Higgs does not couple to any fermions and is dominantly produced by $\mathrm{W} / \mathrm{Z}$ fusion and in association with W/Z. Topcolor Higgs couples to the top quark (the only non-zero fermion coupling) and therefore in addition to $\mathrm{W} / \mathrm{Z}$ fusion and $\mathrm{W} / \mathrm{Z}$ associated production, can also be produced via gluon fusion which increases total production rate significantly.

Note that the terms 'Fermiophobic Higgs' and 'Topcolor Higgs' later in the dissertation refer to the two aforementioned scenarios (classes of models), while elsewhere in the literature they may denote more specific models.

\subsection{Constraints on the Higgs Mass - Previous Results}

As discussed in Section 2.2.1, unitarity considerations lead to an upper bound on the Higgs mass of $\sim 1 \mathrm{TeV}$. Searches for the Higgs boson at Fermilab and CERN in the last decade established also a lower limit on the Higgs mass. In particular, the DØ and CDF experiments performed fermiophobic Higgs searches using $\gamma \gamma$ decay mode $[38,39,40]$ and set 95\% CL lower limits on its mass of 78.5 $\mathrm{GeV}$ and $82 \mathrm{GeV}$, respectively. The corresponding combined limit from LEP is $108.3 \mathrm{GeV}$ at the $95 \%$ CL [41].

A lower limit on the SM Higgs boson mass was set by LEP at $114.4 \mathrm{GeV}$ at the $95 \%$ CL [42] (this limit was lowered in the SUSY models) 


\title{
Chapter 3
}

\section{Fermilab and the DØ Detector}

\author{
Someone will say: it's an old town, \\ but for some reason the houses are all new... \\ Meadows bloom by the roadside. \\ And a regal long-legged stork \\ walks dignified among the early corn... \\ Cars rush, hot-shot drivers twirl their steering wheels. \\ Where are you, people, who used to live in this house? \\ My wide world, what flat lands lie here all around! \\ Lina Kostenko, Collection of Poems
}

In this section we give a brief description of the Fermilab Accelerator (the Tevatron), the DØ Detector and its components, with the main focus on the detector subsystems most relevant to the $h \rightarrow \gamma \gamma$ search, such as tracking and calorimeter systems. Also we discuss briefly the trigger and data acquisition (DAQ) systems.

\section{$3.1 \quad$ Fermilab}

Fermi National Accelerator Laboratory (Fermilab) is located in Batavia, Illinois, 40 miles west of Chicago. Fermilab's 6,800-acre site was originally home to farmland, and to the village of Weston. 
It was commissioned by the US Atomic Energy Commission in 1967 [43]. Robert R. Wilson was the founding director of the laboratory [44]. The facility has been built and operated by the Universities Research Association. Originally named National Accelerator Laboratory, it has been renamed in 1974 in honor of Enrico Fermi. Fermilab hosted two major discoveries: that of the bottom and top quarks (in 1977 and 1995, respectively). Also, the first direct observation of tau neutrino was announced by Fermilab in 2001. The top quark and tau neutrino were the last two of the Standard Model fermions to be observed.

\subsection{Accelerator}

This subsection is based on references [45, 46, 47]. The Tevatron is a $p \bar{p}$ collider with a center-of-mass energy of $1.96 \mathrm{TeV}$ located at Fermilab. It started its operation in 1987 [48] (in 1988 the $1.8 \mathrm{TeV}$ collision energy has been achieved). The Run I high energy physics program (1992-1996) delivered $\sim 130 \mathrm{pb}^{-1}$ to each of the two collider detectors. The collider has been upgraded since, and currently the Run II is underway. This is the highest energy collider program until the Large Hadron Collider at CERN will start its operation at the end of this decade.

Fermilab Run II accelerator complex is a chain of increasing energy accelerators, as shown in Figure 3.1. The proton beam originates as negatively charged hydrogen ions $H^{-}$enter the preaccelerator (Cockroft-Walton). There the ions are accelerated to $750 \mathrm{keV}$, then bunched and led into a 500 foot $(150 \mathrm{~m})$ long linear accelerator (LINAC). In LINAC the ions are accelerated to 400 $\mathrm{MeV}$ (by oscillating electric fields), after which they are passed through a carbon foil. The foil strips both electrons from the hydrogen nucleus leaving the naked proton. The protons are steered into the Booster, a 1570 foot $(500 \mathrm{~m})$ circumference fast-cycling synchrotron ring. In the Booster the protons are focused into a closed circular orbit by a series of quadrupole magnets, accelerated to an energy of $8 \mathrm{GeV}$ via a set of RF cavities, and collected into bunches before their insertion into the Main Injector.

The Main Injector is a long synchrotron ring 2 mile $(3 \mathrm{~km})$ in circumference that accelerates protons to an energy of $150 \mathrm{GeV}$ before injecting them into the Tevatron. Anti-protons are also 


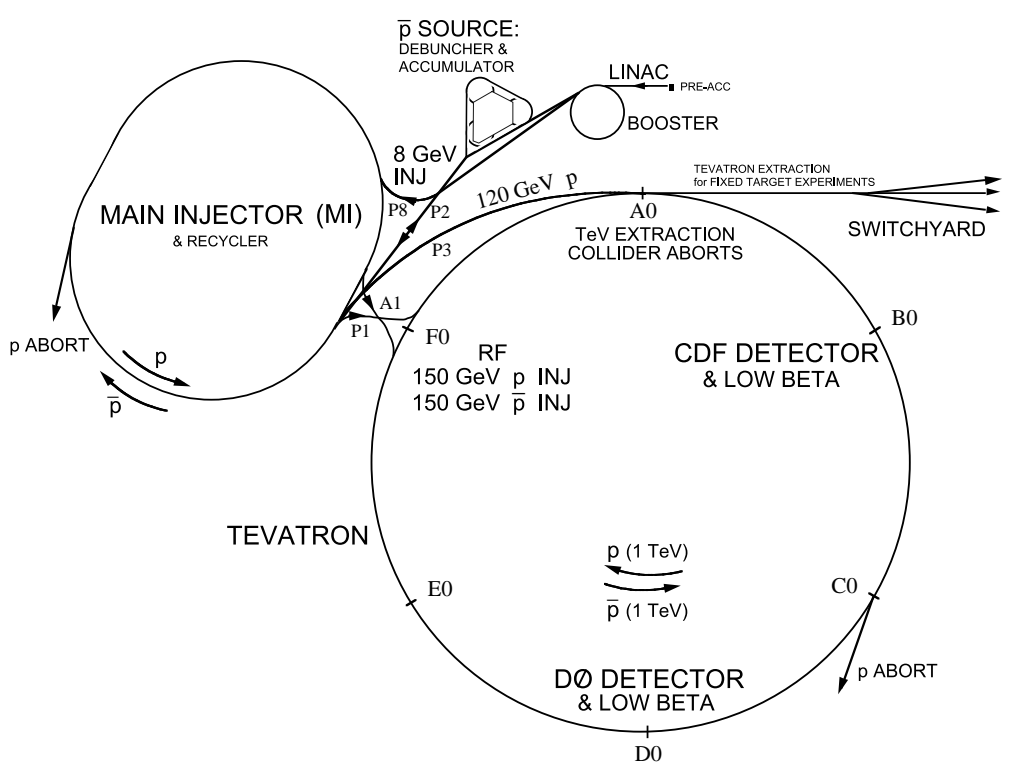

Figure 3.1: Fermilab accelerator complex

produced in the Main Injector. The $120 \mathrm{GeV}$ protons are extracted and made to collide with a nickel target. These collisions produce many secondary particles, among which are anti-protons, approximately one for every 105 protons. The emerging anti-protons are focused and collected into the storage rings, the Debuncher and Accumulator, where they are cooled, bunched, and stored before returning back to the Main Injector to be accelerated to $150 \mathrm{GeV}$ for injection in the Tevatron.

Bunched protons and anti-protons are inserted into the Tevatron ring where they are further accelerated with RF cavities and moved together to collide at $1.96 \mathrm{TeV}$ center-of-mass energy in two interaction regions, $\mathrm{B} \varnothing$ and $\mathrm{D} \varnothing$ where the CDF and $\mathrm{D} \varnothing$ detectors are located. To bend the protons and anti-protons into a circular orbit the Tevatron uses superconducting magnets with a field strength of $4.2 \mathrm{~T}$. The circumference of the Tevatron tunnel is 4 miles $(6 \mathrm{~km})$. The time interval between (bunch) collisions is $396 \mathrm{~ns}$.

Currently about $220 \mathrm{pb}^{-1}$ of data have been collected by each experiment (for comparison, in Run I of the Tevatron total integrated luminosity per experiment was $\sim 130 p b^{-1}$ ). Current goal of the Tevatron is to collect between 4.4 and $8.6 \mathrm{fb}^{-1}$ by 2009 . 


\subsection{DØ Detector}

The DØ Detector is a multipurpose nearly-hermetic $4 \pi$ particle detector. It has been constructed to study proton-antiproton collisions at $\sqrt{s}=2 \mathrm{TeV}$ in the Fermilab Tevatron Collider [49]. The experiment was first provisionally approved in 1983 and the full conceptual design report was prepared a year later [68]. It has played a crucial role in modern experimental high energy physics since its first successful operation in 1992. For example, in 1995 the top quark was discovered by the DØ Collaboration together with CDF $[7,8]$. The prime physics focus of the DØ experiment in Run II is the study of high mass states and large $p_{T}$ phenomena, as well as vigorous B-physics progarm. The former include the precision study of the top quark and the $\mathrm{W}$ and $\mathrm{Z}$ bosons to provide sensitive tests of the Standard Model, searches for the Higgs boson and new phenomena beyond the Standard Model, and various studies of perturbative and non-perturbative QCD.

A $3 \mathrm{D}$ cut-away view of the DØ detector [46] is shown in Figure 3.2.

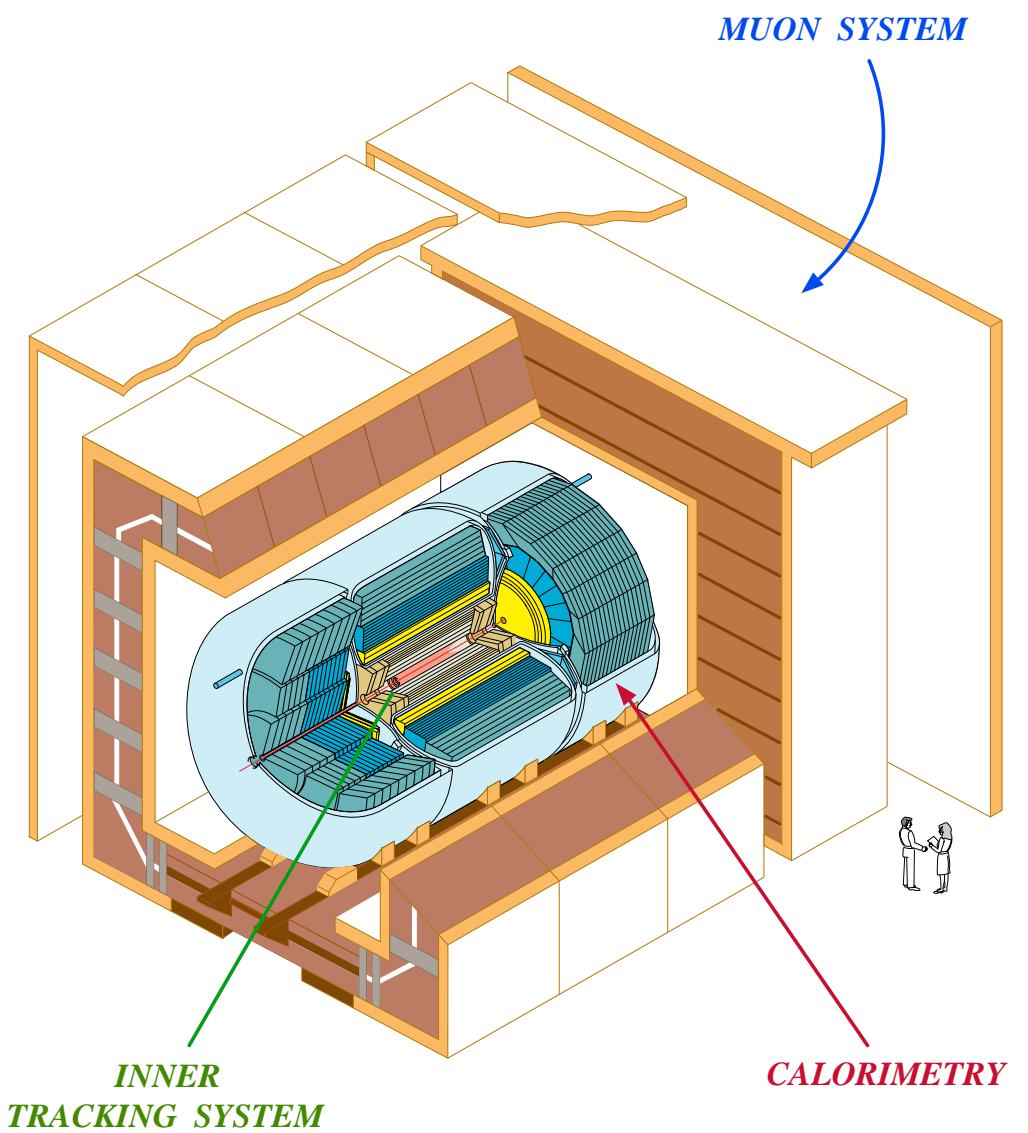

Figure 3.2: 3D view of the $\mathrm{D} \varnothing$ Detector. 


\subsection{Coordinate System and Useful Variables}

$\mathrm{D} \emptyset$ uses a right-handed coordinate system, in which the z-axis is along the proton direction and the y-axis points upward [6]. The angles $\phi$ and $\theta$ are the azimuthal and polar angles, respectively ( $\theta=0$ along the proton beam direction). For ultra-relativistic particles instead of the polar angle it is more convenient to use pseudorapidity $\eta$, defined as

$$
\eta=-\log (\tan (\theta / 2))
$$

It approximates the true rapidity $y$ (rapidity intervals are Lorentz invariant)

$$
y=\frac{1}{2} \log \frac{E+p_{z}}{E-p_{z}}
$$

for finite angles in the limit that $m / E \rightarrow 0$.

Kinematic variables that are commonly used for the analyses at DØ are transverse energy ${ }^{1} E_{T}=$ $E \sin \theta$ and transverse momentum $p_{T}=p \sin \theta$. Such choice of variables is motivated by the fact that the center-of-mass energy $(\sqrt{\hat{s}})$ of the scattering in $p \bar{p}$ collisions is not fixed [50]. This is a consequence of the parton structure of a nucleon [16]. The partons (quarks and gluons) carry a fraction of the total nucleon energy. Scattering of partons of different energy results in the center of mass frame that does not coincide with the lab frame; also the total energy released in the collision is only a fraction of the total energy of the colliding beams.

Moreover, although the collision energy of the nucleons as a whole is fixed, the energy balance can not be used to analyze the outcome of the collision, since a significant fraction of energy escapes the detector as the nucleon remnants (spectators) carry it away down the uninstrumented beampipe. However, the transverse energy balance can be used since it is known to be zero before the collison and its undetectable fraction is negligible.

The DØ detector consists of the three major subsystems :

\footnotetext{
${ }^{1}$ Note that the term transverse energy can have two meanings. At $\mathrm{D} \varnothing$ it is typically used for $E_{T}=E \sin \theta$. This meaning is implied in the dissertation. However this term may also be used to denote the energy in the frame of zero longitudinal momentum (which is not equal to $E \sin \theta[50]$ )
} 
1. Tracking System;

2. Calorimeter System;

3. Muon System.

The full list of the DØ detector components includes:

- Preshower detectors;

- Intercryostat and Massless Gap Detectors ;

- Luminosity Monitor;

- Forward Proton Detector (FPD);

- Trigger Framework;

- Data Acquisition System (DAQ).

Figure 3.3 shows the $r-z$ view of the $\mathrm{D} \varnothing$ detector [46] indicating its major components.

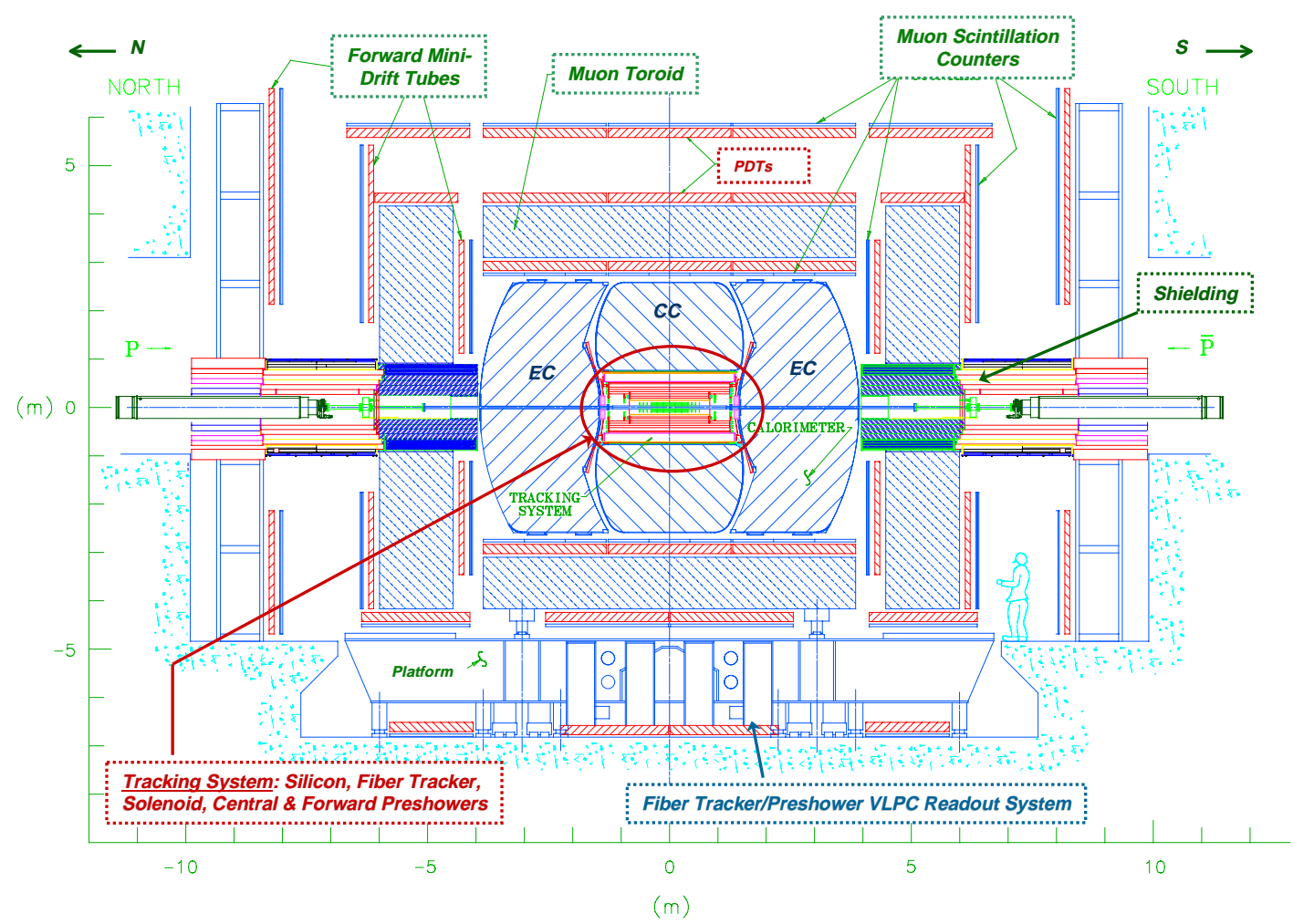

Figure 3.3: $r-z$ view of the DØ Detector. 


\subsection{Tracking System}

The goals of the $\mathrm{D} \varnothing$ tracking system are:

- detection of charged particles over large range of pseudorapidity $(\eta \approx \pm 3)$;

- charged particle momentum measurement in the solenoidal magnetic field;

- secondary vertex measurement for identification heavy flavors.

The DØ tracking system consists of the two subsystems: the Silicon Microstrip Tracker (SMT) and the Central Fiber Tracker (CFT). The $r-z$ view of the quadrant of the tracking system is shown in Figure 3.4.

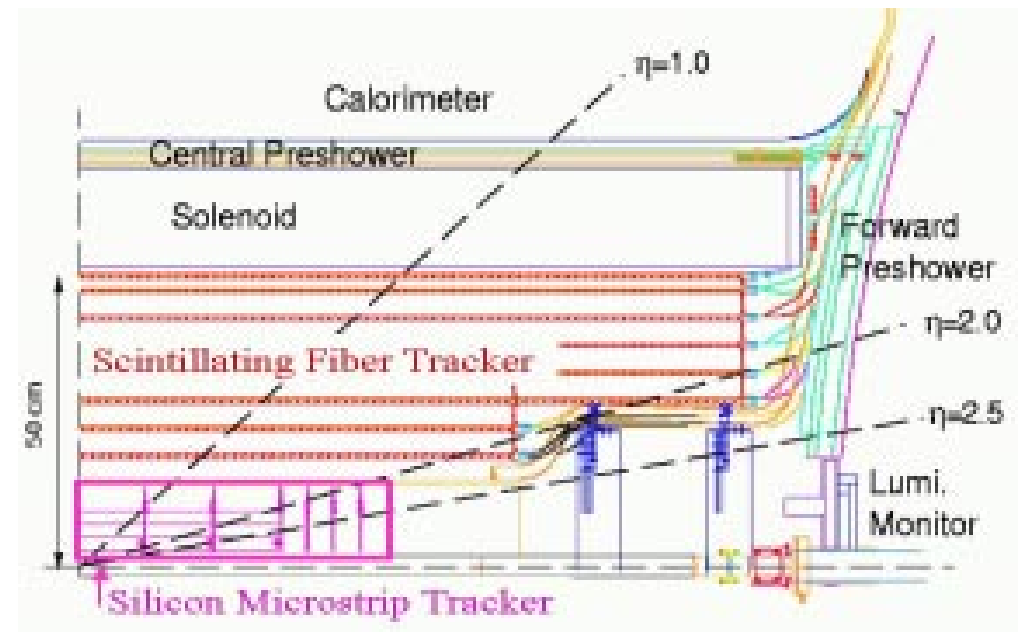

Figure 3.4: DØ tracking system (the quadrant $r-z$ view) (Adapted from [51])

\subsubsection{Silicon Tracker}

The Silicon Microstrip Tracker (SMT) [52] is the high resolution part of the tracking system and is the first set of detectors encountered by the particles emerging from the collision. It was included in the DØ Detector as part of the Run II upgrade [53]. The choice of a silicon semiconductor tracking device was motivated by the following properties of the silicon [54]:

- low ionization energy (good detectable signal);

- long mean free path (good charge collection efficiency); 
- high mobility (fast charge collection);

- low Z (low multiple scattering);

- well-developed technology.

The Silicon Tracker was designed as a hybrid system consisting of barrel detectors measuring primarily the $r-\phi$ coordinate and disk detectors which measure $r-z$ as well as $r-\phi$. Such type of design was motivated by the fact that the interaction point is distributed over the $z$ coordinate with $\sigma_{z}=28 \mathrm{~cm}$ due to the structure of the colliding proton and antiproton bunches. Therefore it is difficult to design a detector such that the tracks are generally perpendicular to the detector surfaces at all $\eta$, so the hybrid system provides a solution. In this type of system, the tracks for high $\eta$ particles are reconstructed in three dimensions primarily by the disks, while particles at small $\eta$ are detected primarily by the barrels. The interspersed disk and barrel design is shown in Figure 3.5 .

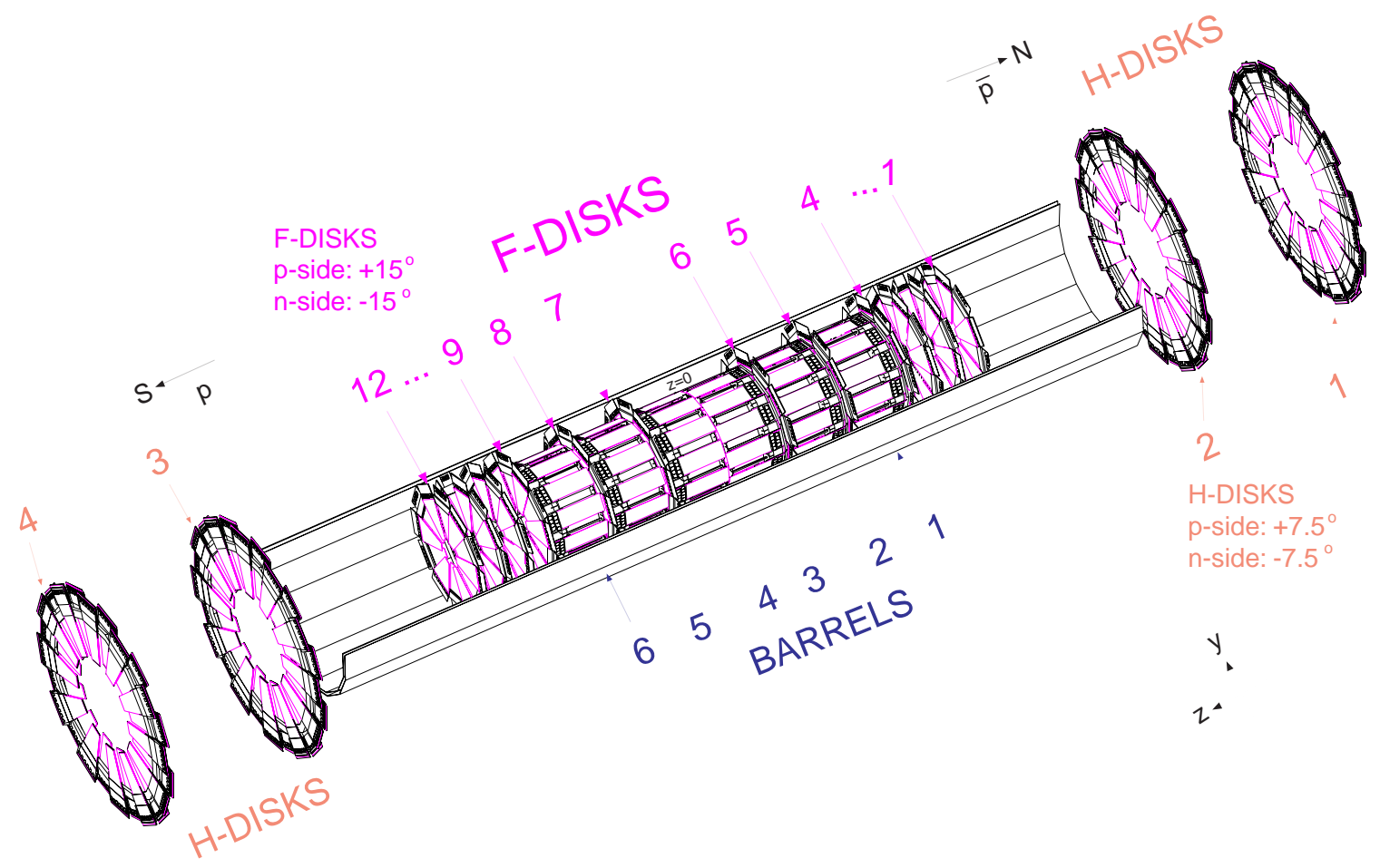

Figure 3.5: the SMT design structure

Conceptually the barrels and the disks have the same structure. The basic detector unit [51] consists of: 
1. Silicon microstrip sensors;

2. SVX II front end readout chips;

3. High density interconnect (HDI) circuit with Kapton strip cable;

4. Supporting Rochacell-carbon and beryllium components.

In the case of a barrel detector such unit is called a ladder. Three types of ladders are distinguished based on the number of readout chips and types of silicon sensors : 3-chip, 6-chip and 9-chip ladders. A photograph of a 3-chip ladder is shown in Figure 3.6. It is a $12 \mathrm{~cm}$ long detector consisting of two silicon sensors. Each sensor is a one-sided sensor with $50 \mu \mathrm{m}$ strip pitch. The two sensors are wire-bonded together on the strip side and supported by the rochacell-carbon fiber support rails on the other side (not shown in the photograph). The strips are connected to the SVX II chips which are mounted on the HDI circuit. The HDI circuit is laminated onto a $300 \mu \mathrm{m}$ thick beryllium plate and glued to the surface of the detector.

The ladders are mounted on bulkheads to form patterns of concentric layers surrounding the interaction point. Such a pattern allows to reconstruct a track of a charged particle based on the hit position at each layer. A bulkhead is a mechanical structure made out of beryllium. A schematic diagram of a bulkhead is shown in Figure 3.7 .

In addition to providing mechanical support, it also serves as an enclosure for the integrated coolant channel system. The cooling $\left(<10^{\circ} \mathrm{C}\right)$ is needed for optimal mode of operation of silicon detectors. Figure 3.8 shows ladders mounted on the bulkhead structure. The SMT is a complicated device with a high level of performance requirements for the first time used in D $\emptyset$. For example the total number of readout channels is 792,576 [51] (for comparison, in the calorimeter system this number is $55,000[56])$.

Since the SMT is rather compact compared with other detector subsystems, its construction was a challenging enterprise in which many people were involved with a wide variety of skills and expertise. For example at the stage of the assembly of a barrel several goals had to be achieved:

1. Protecting the production stage quality of a ladder (very small forces applied and chemical 


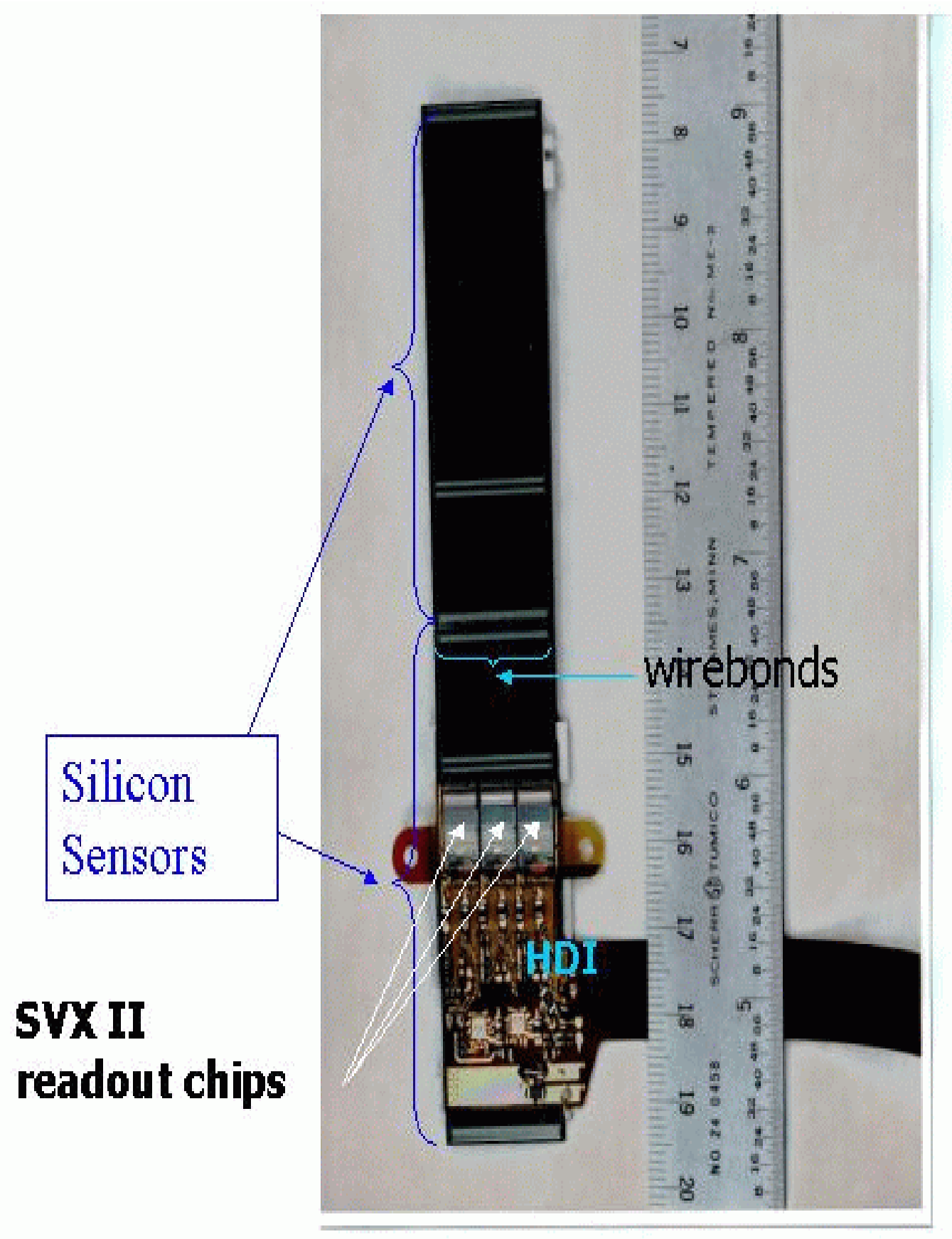

Figure 3.6: DØ 3-chip ladder photograph. (Adapted from [55]) 


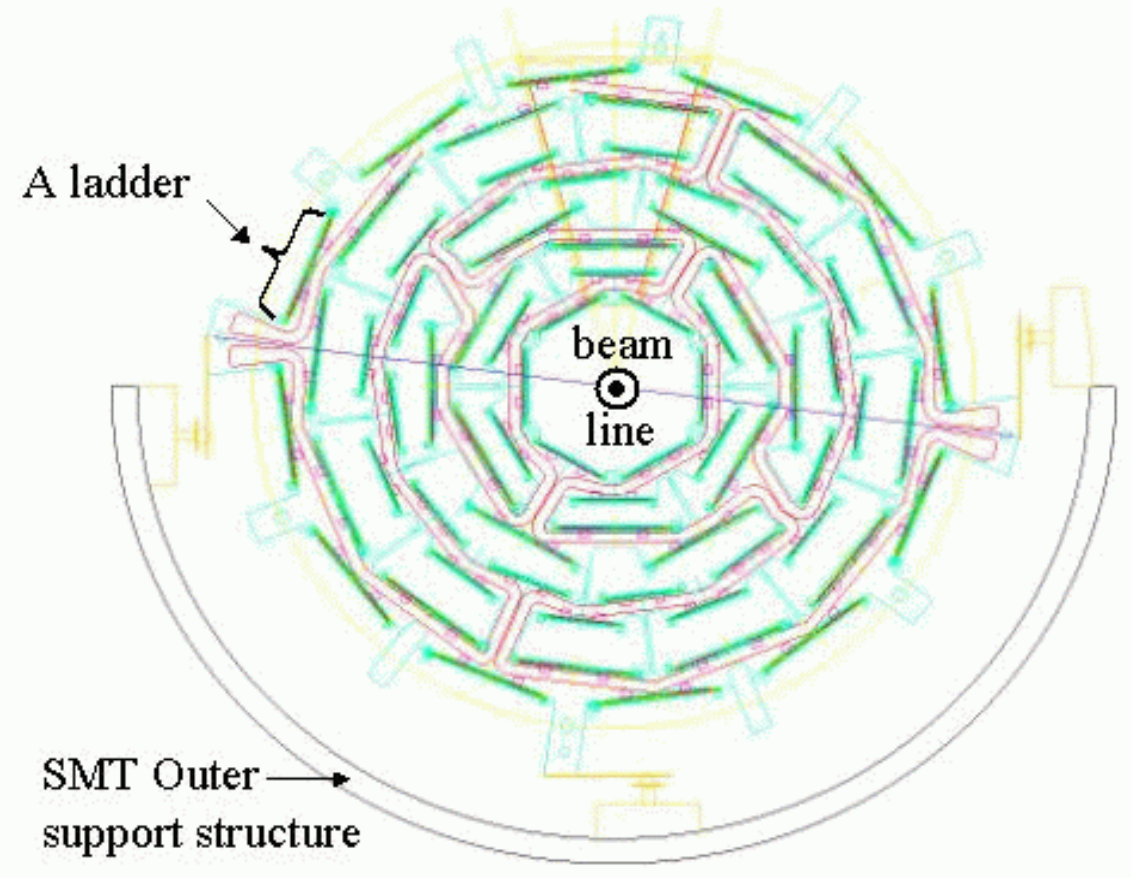

Figure 3.7: the $x-y$ view of the bulkhead

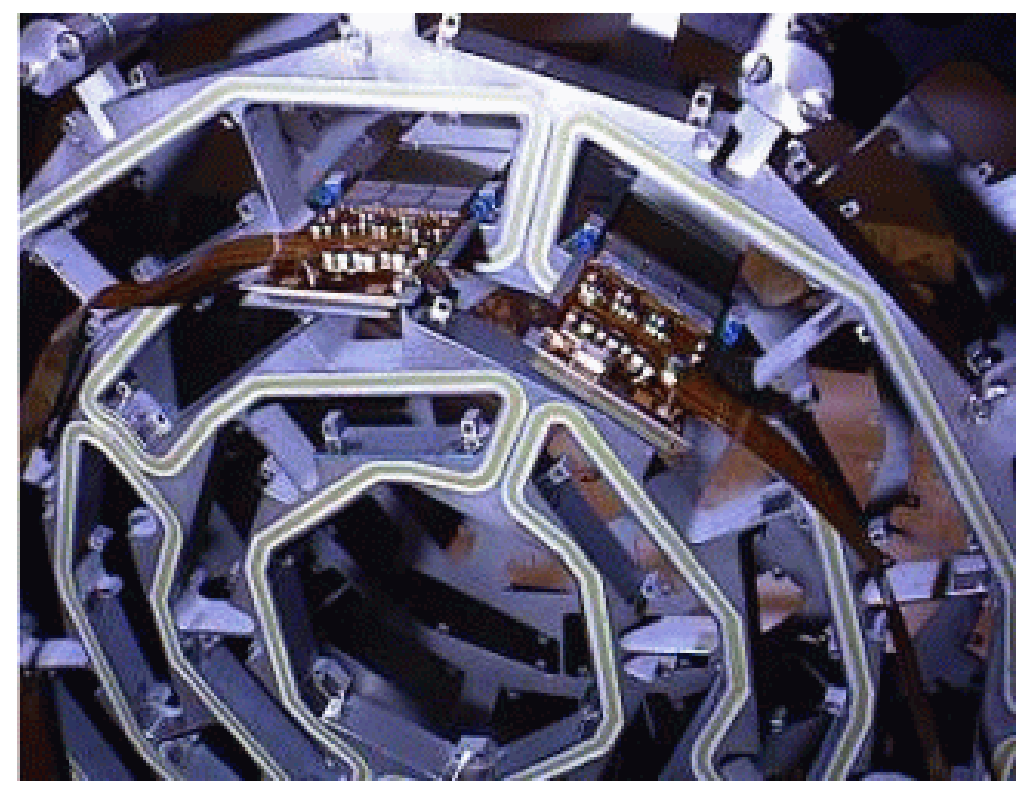

Figure 3.8: A bulkhead with two ladders installed 
changes in the environment may affect the ladder properties dramatically);

2. Retaining the quality of the readout electronics performance as a ladder is moved out of the production and testing enclosure;

3. Precise mechanical positioning of ladders onto a bulkhead that should be retained at the subsequent stages of the SMT construction and commissioning such as combining barrels with disks into enclosing support structure, installing the SMT into the DØ Detector in the experimental hall;

4. Allowing access for the cabling and cooling system outlets.

These objectives were of different nature but had to be achieved simultaneously. This situation imposed at times seemingly conflicting requirements on the assembly procedure which made it a creative and exciting task [57].

\subsubsection{Central Fiber Tracker}

The scintillating fiber tracker surrounds the silicon detector and covers the central pseudo-rapidity region [53] as shown in Figure 3.4. The fiber tracker serves two main functions :

1. Together with the silicon detector it enables track reconstruction and momentum measurement for all charged particles within the range $|\eta|<2.0$. Combined hit information from the two tracking systems allows to improve the overall tracking quality.

2. The fiber tracker provides fast "Level 1" track triggering within the range $|\eta|<1.6$

A total of about 74,000 scintillating fibers are mounted on eight concentric cylinders. Each of the cylinders supports a doublet layer of fibers oriented in the axial direction, parallel to the beam line and two doublet layers of fibers that are oriented at $3.0^{\circ}$ ) stereo angles.

The basic detection element is the multi-clad scintillating fiber. The inner polystyrene core of the fiber is surrounded by two layers of cladding (acrylic and fluoro-acrylic). These three materials have indices of refraction of $1.59,1.49$, and 1.42 , respectively. The fiber diameter is $835 \mu \mathrm{m}$ and 
each cladding is $15 \mu \mathrm{m}$ thick. The fiber scintillates in the yellow-green part of the visible spectrum, with the peak emission wavelength near $530 \mathrm{~nm}$.

Figure 3.9 shows the $r-z$ view of the scintillating fiber tracker and the end view of the tracker fibers [46].

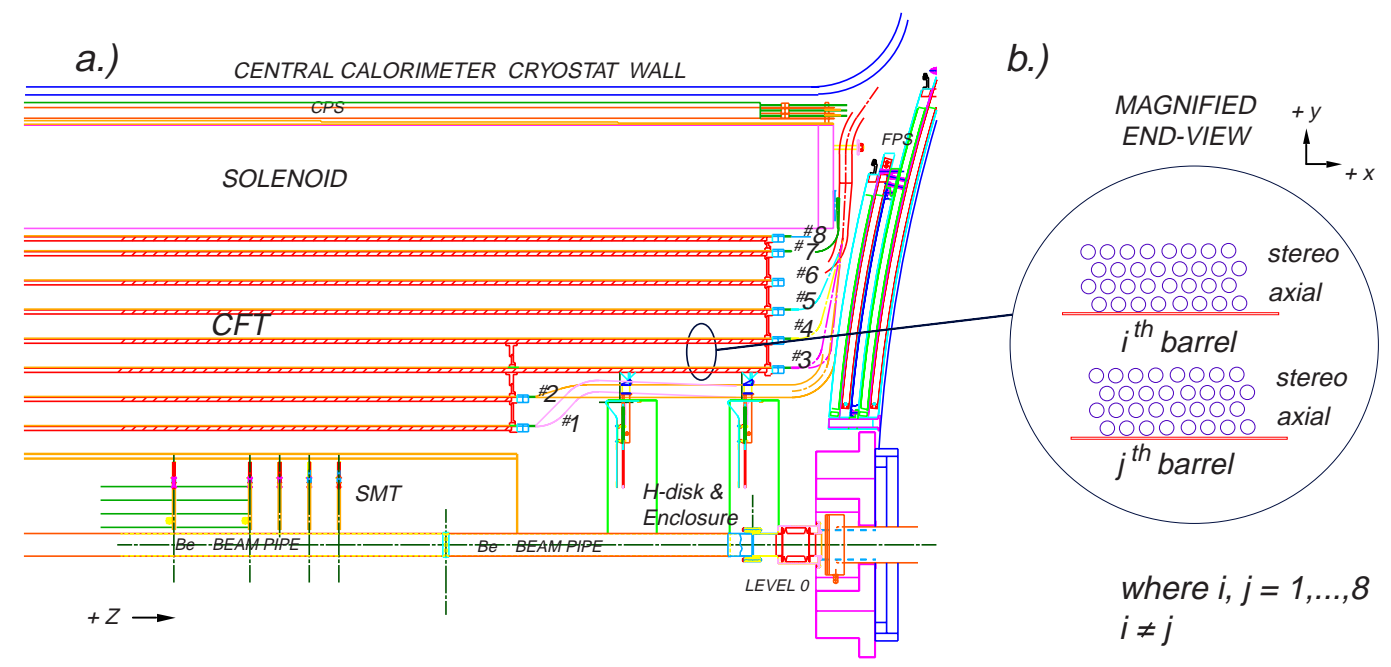

Figure 3.9: the $r-z$ view of the scintillating fiber tracker with the end view of the fibers.

Scintillating fibers are mated to the waveguides by plastic, diamond-finished optical connectors. These waveguides conduct the scintillation light to photodetectors, which are the Visible Light Photon Counters (VLPC) (e.g. [58]). VLPC is a variant of the solid-state photomultiplier, operating at $\sim 9^{\circ} \mathrm{K}$.

\subsection{Calorimeter System}

High-energy is often a code word for high-calorie

a tip from American Council on Exercise posted in Fermilab's gym

\subsubsection{Calorimetry Basics}

Calorimeter detectors are used in high energy physics for : 
1. Measurement the particle energy;

\section{Particle identification.}

In addition they can be used for determining the decay vertex position in cases when the tracking information is not available or limited.

Calorimeters are devices that entirely absorb incident particles by making them interact in the detector material [59]. In the process of optimally-controlled absorption, cascades of interactions occur, which are called showers. Most of shower energy is converted into heat (calor is Latin for heat). Part of the energy is released in the form of the recordable signal such as scintillating light or ionization.

From the construction point of view two types calorimeters are distinguished :

\section{Homogeneous calorimeters;}

2. Sampling calorimeters.

In homogeneous calorimeters the role of absorption and signal creation is carried by the same material, typically a pure or doped heavy crystal (like NaI or CsI), or a composite material (like lead glass). They require more space than sampling calorimeters. The growing and machining of large homogeneous crystals can be problematic. They are, therefore, mostly used for moderate energy electromagnetic calorimetry, with high precision requirements.

In (heterogeneous) sampling calorimeters, the absorber material is inactive and interspersed with layers of active (signal-producing) material, typically liquid or solid scintillator. Inactive materials typically are lead, iron, copper, uranium, or combination thereof. The ratio of energy loss in active and inactive material typically is of the order 1:10.

If the sampling of a signal contains adequate detail of the absorption process, the original phenomenon can be inferred from it, e.g. and incident particle's energy or the energy dissipation profile in a part of geometrical space. As shower development is largely independent of the charge of the incident particle, calorimeters also are unique instruments for measuring energy of neutral particles. They also can be used for detecting and measuring particle jets, in which mixtures of neutral and 
charged particles are present at small spatial separation. Tracking devices, on the other hand, are not useful (or only marginally useful) for either neutral particles or jets.

Among the relevant showering processes there are two major categories: electromagnetic showers, which are caused by incident electrons and photons, and hadronic showers, which are induced by hadrons. Hadronic showers generally also contain electromagnetic shower components, but, in addition, a large variety of specifically hadronic phenomena determine their parameters. For a given energy of an incident particle a hadronic shower tends to be of a larger size than the electromagnetic one (please refer to the discussion of electromagnetic and hadronic shower properties in Chapter 4). This is reflected in the design of the calorimeters. A typical calorimeter system in a multipurpose collider detector consists of two subdetectors: the electromagnetic calorimeter and the hadronic calorimeter. Electromagnetic calorimeter is more compact; it encloses the volume closer to the interaction point in which typically the tracking detectors are placed, while a hadronic calorimeter covers the outer region of the detector. Fluctuations in the elecromagnetic content of the hadronic shower lead to difficulties in energy measurement. Another complication comes from the fact that a sizeable amount of the available energy is converted into excitation or break-up of the nuclei, of which only a fraction will result in detectable ('visible') energy. In other words the $e / h$ ratio (of electromagnetic and hadronic response) is not equal to one. Attempts were made to make it as close to one as possible by means of compensation. The original idea was to use uranium as the absorber material [59]; this would contribute an additional, i.e. compensating signal due to nuclear fission caused by nuclear excitation. Several calorimeters were built with $e / h=1 \pm 0.02$ (including the DØ

Calorimeter). Later it was understood that fission is only one of the compensating mechanisms in sampling calorimeters. The photon absorption in the (high-Z) material plays a significant role, and so does the conversion of low-energy neutrons into signal, e.g. by detection of de-excitation photons.

\subsubsection{D $\varnothing$ Calorimeter}

DØ Calorimeter is a compensating sampling calorimeter in which liquid argon is used as an active medium and depleted uranium (as well as copper and steel) as an absorber material $[49,68,69]$. 
The design of the calorimeter system must allow for some degree of access to the Central Detectors. This can be achieved by using more than one containment vessel (cryostat). The solution that was chosen by $\mathrm{D} \emptyset$ is shown schematically [46] in Figure 3.10 .

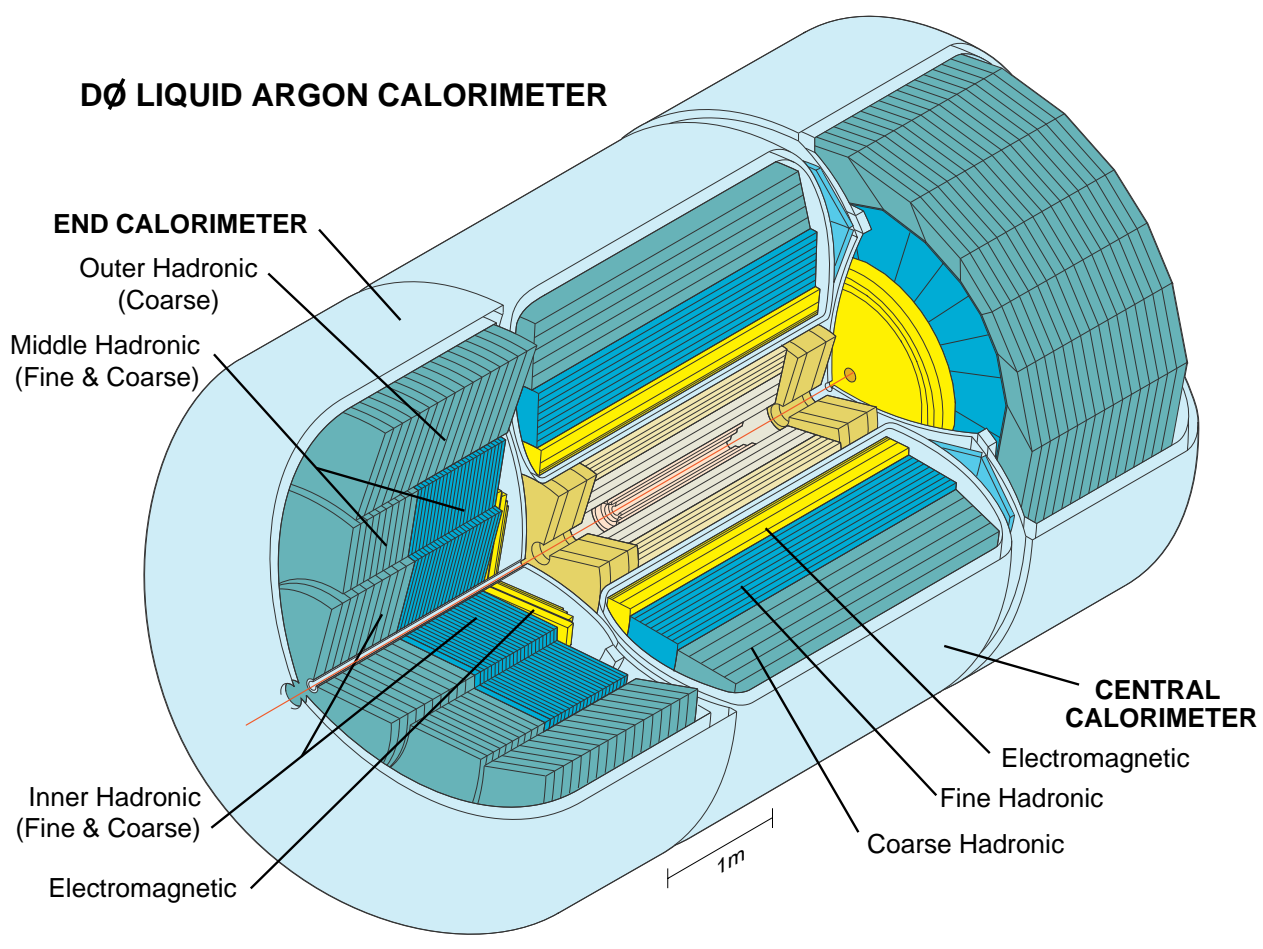

Figure 3.10: DØ Calorimeter.

There are three distinct modules in the DØ calorimeter system: Central Calorimeter (CC), which covers roughly the $|\Delta \eta| \leq 1.2$ region and a pair of end calorimeters (EC North and EC South), which extend the $\eta$ coverage out to $|\eta| \approx 4.5$. The boundary between $\mathrm{CC}$ and EC was chosen to be approximately perpendicular to the beam direction. This choice was shown to introduce less degradation in the missing transverse energy measurement.

The dimensions of the calorimeters are set according to the need for adequate containment of the shower energy. In addition, since the calorimeters are embedded in a multidetector system, the following factors must be taken into consideration:

- the need for sufficient tracking coverage in front (inside) of the calorimeter;

- the requirements of magnetic measurement of muon momenta outside the calorimeter; 
- the size of the experimental hall.

The resulting design has three distinct types of modules in both $\mathrm{CC}$ and $\mathrm{EC}$ :

1. electromagnetic section (EM) with relatively thin uranium absorber plates;

2. fine-hadronic section (FH) with thicker uranium plates;

3. coarse-hadronic section $(\mathrm{CH})$ with thick copper or stainless steel plates.

The EM modules consist of four separate layers EM1, EM2, EM3, EM4. These are radial layers for the $\mathrm{CC}$ and for the EC they are z-layers (see section 3.4 that explains DØ coordinate system). Each layer uses $3 \mathrm{~mm}$ or $4 \mathrm{~mm}$ thick nearly pure depleted uranium plates [70] as an absorber. The FH modules consist of three or four layers that have $6 \mathrm{~mm}$ thick uranium-niobium (2\%) alloy absorber plates. The outer $\mathrm{CH}$ section has only one layer that uses relatively thick (46.5 mm) plates of either copper (CC) or stainless steel (EC).

The depth of each layer for the three calorimeter sections is shown in Table 3.1 in the units of radiation lengh $X_{0}$ and absorption length $\lambda[39]$.

\begin{tabular}{cccc}
\hline & EM & FH & CH \\
\hline CC Depth & $2,2,7,10 X_{0}$ & $1.3,1.0,0.9 \lambda$ & $3.2 \lambda$ \\
EC Depth & $0.3,2.6,7.9,9.3 X_{0}$ & $1.2,1.2,1.2 \lambda$ & $3.6 \lambda$ \\
\hline
\end{tabular}

Table 3.1: The depth of the calorimeter layers.

From the readout point of view each layer represents a discrete set of readout cells. A typical transverse sizes of a cell are $\Delta \eta=0.1$ and $\Delta \phi=2 \pi / 64 \approx 0.1$ (the EM3 layer, however, is twice as finely segmented in both $\eta$ and $\phi$ to allow for more precise location of the EM shower centroids). A set of cells (one cell from each layer) that are aligned along the outward direction (approximate direction of a shower development) constitute a tower. The readout tower geometry is shown in Figure 3.11. This is a 'pseudo-projective' geometry. The term 'pseudo-projective' refers to the fact that the centers of cells of increasing shower depth lie on the rays projecting from the center of the detector, but the cell boundaries are aligned perpendicular to the absorber plates. 


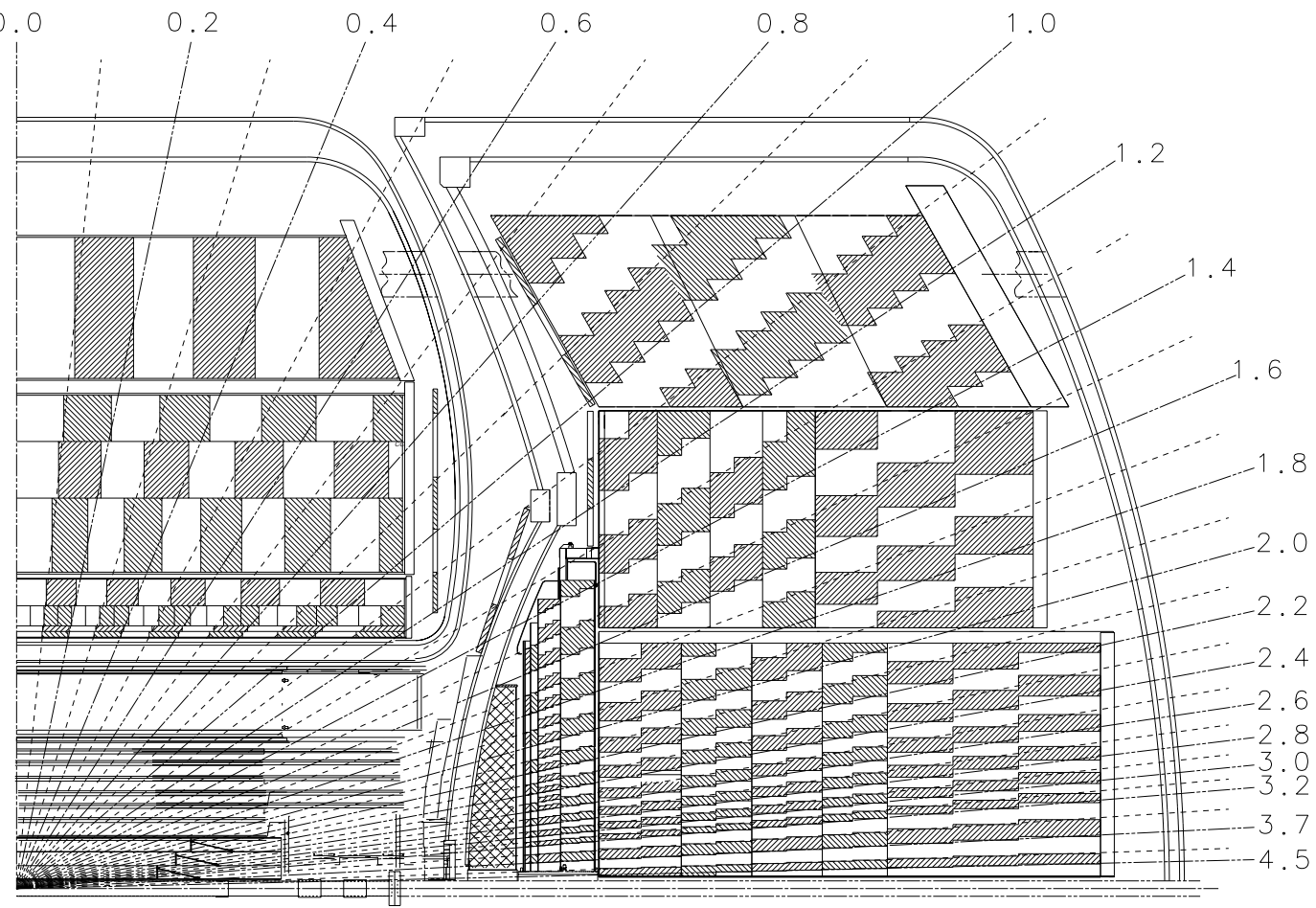

Figure 3.11: Pseudo-projective geometry of the DØ Calorimeter.

A cell (readout cell) is a combination of several adjacent unit cells. A schematic view of the calorimeter unit cell [6] is shown in Figure 3.12. The gap between the adjacent absorber plates is filled with liquid argon. The electron-ion pairs created via the ionization of the liquid argon by charged particles from a shower are collected by the electrodes in the presence of a strong electric field. Metal absorbers are used as ground electrodes (cathodes), and the readout boards at +2.0 to $2.5 \mathrm{kV}$, located in the center of the gaps, serve as anodes. Each board in most of the modules is a sandwich of copper readout pads between the two $0.5 \mathrm{~mm}$ plates of G10 plastic covered with a resistive epoxy coating. High voltage is applied to the entire resistive coat, and the charge collection in this coat induces a charge on the copper readout pads via capacitive coupling. To detect signals that can be very small, several pads are ganged together in depth to form a readout cell. The electronics receives an analog signal from a readout cell which is proportional to the energy deposited by the shower in the active media [45]. The simplified calorimeter data flow path diagram is shown in Figure 3.13 and in a more detailed diagram in Figure 3.14. Coaxial cables carry the signal to a feed-through port, which allows to pass it through the cryostat. The feed-through boards reorganize signal form 


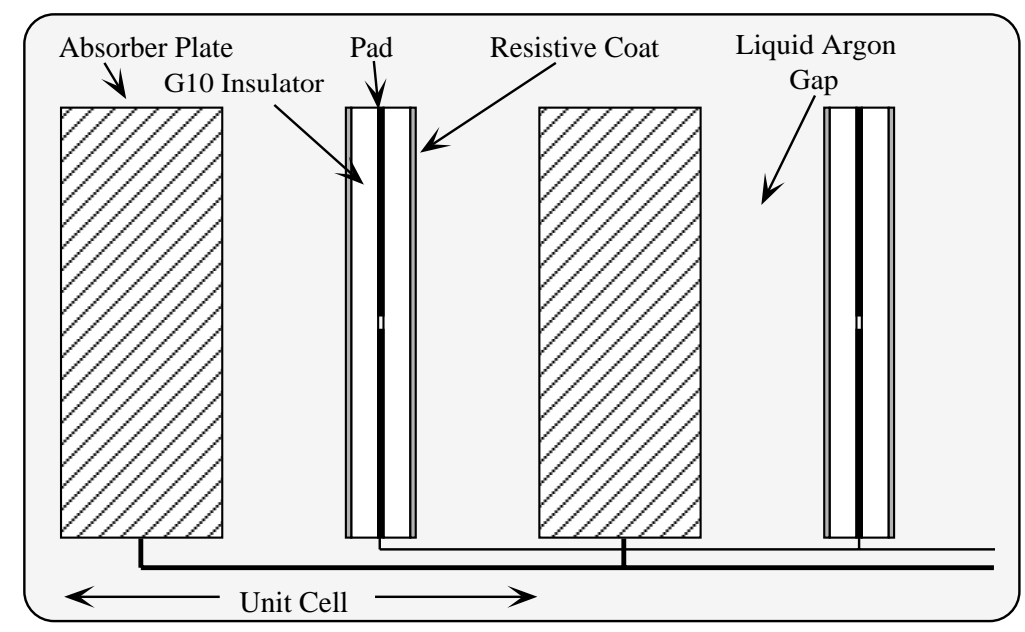

Figure 3.12: Calorimeter unit cell

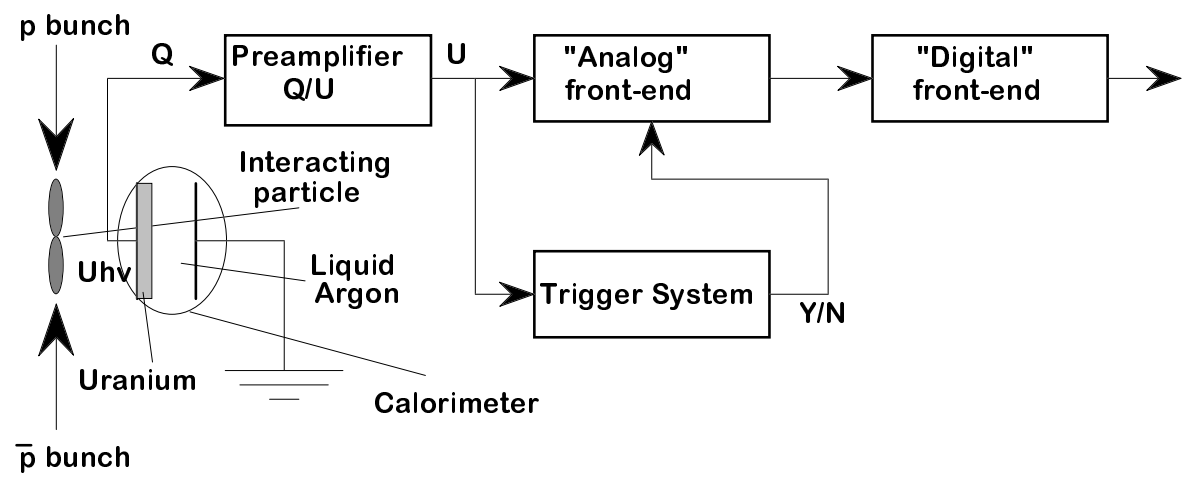

Figure 3.13: Simplified calorimeter data flow diagram. 


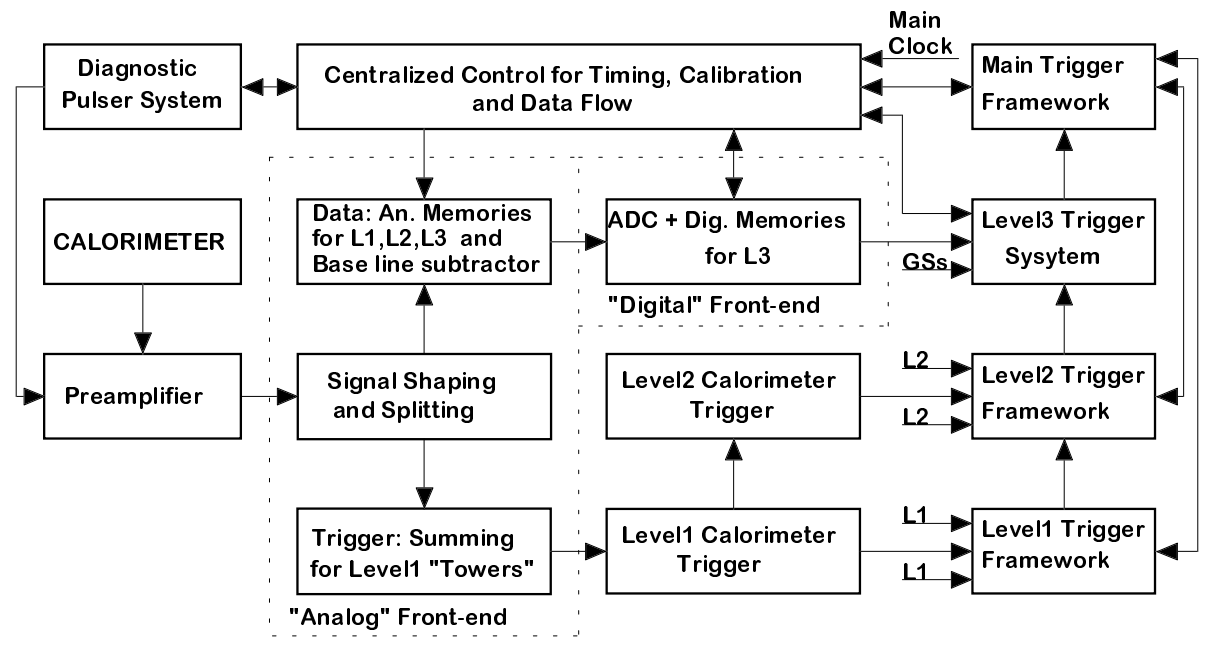

Figure 3.14: Full calorimeter data flow diagram.

the module-structure scheme to the physcis scheme in which the readout channels are arranged in the pseudo-projective $\eta-\phi$ towers [56]. The signal is then conducted to the charge-sensitive preamplifiers. The preamplifiers integrate the pulse over time to produce proportional voltages. The preamplifier outputs go through 30 meter coaxial cables to the signal shapers. After the signal is shaped, the data flow splits in two paths. One path takes the data to the Level 1 calorimeter trigger. Another (the precision readout) path leads to the baseline subtraction system (BLS). The BLS performs the cell signal sampling just before and after the beam crossing and takes the difference between the two. This is done in order to separate the signal coming from the event to be recorded from the noise as well as previous collision remnants. The baseline-subtracted output is stored in a 'sample and hold' circuit. Following the trigger decision to keep the event, 'sample and hold' outputs are read out and digitized by the Analog-to-Digital Converters (ADC). The digitized signal from the calorimeter is then merged with the signal information from the other detector systems to form an event.

\subsection{Intercryostat and Massless Gap Detectors}

As shown in Figure 3.11 in the region between the $\mathrm{CC}$ and EC calorimeters there is a large amount of uninstrumented material. Most of this material is due to the cryostat walls, calorimeter support, 
and cabling for the detector readout [39, 49]. To better sample this region, scintillation detectors have been mounted on each of the EC cryostat walls, facing the gap. Each intercryostat detector (ICD) consists of 384 scintillator tiles of size $\Delta \eta=\Delta \phi=0.1$ exactly matching the calorimeter cells. In addition separate single-cell structures, called massless gaps, are installed in both CC and EC calorimeters. Together, the ICD and massless gaps provide a good approximation to the standard DØ sampling of hadron showers.

\subsection{Preshower Detectors}

Preshower detectors (Central Preshower (CPS) and Forward Preshower (FPS)) were designed for the Run II upgrade to aid electron and photon identification, as well as to correct the electromagnetic energy for the effects of the uninstrumented solenoid material. The preshower detectors were in commissioning stage when the data for the current $h \rightarrow \gamma \gamma$ analysis were collected and thus have not been used in the analysis.

The CPS [53, 71] functions both as a calorimeter (by early energy sampling) and as a tracker (by providing precise position measurements). The cylindrical detector is placed in the $51 \mathrm{~mm}$ gap between the solenoid coil and the central calorimeter cryostat at a radius of $72 \mathrm{~cm}$, and covers the region $-1.2<\eta<1.2$. The detector consists of three layers of scintillating strips arranged in axial and stereo views. The stereo angles for the two stereo layers are $\pm 23^{0}$. Figure 3.15 shows the the cross-sectional end $(x-y)$ and side $(r-z)$ views of the CPS detector.

The FPS detector is the forward region counterpart of the CPS. It covers the $1.4<|\eta|<2.5$ region. The FPS detectors are mounted on the inner face of each of the End Calorimeter (EC), as shown in Figure 3.16.

The same technology as for the CPS is used: triangular scinitllator strips with embedded wavelength-shifting fibers, read out by the VLPCs.

The FPS (and CPS) share the same (VLPC) readout system with the Scintillating Fiber Tracker, which was mentioned in Section 3.5.2. 

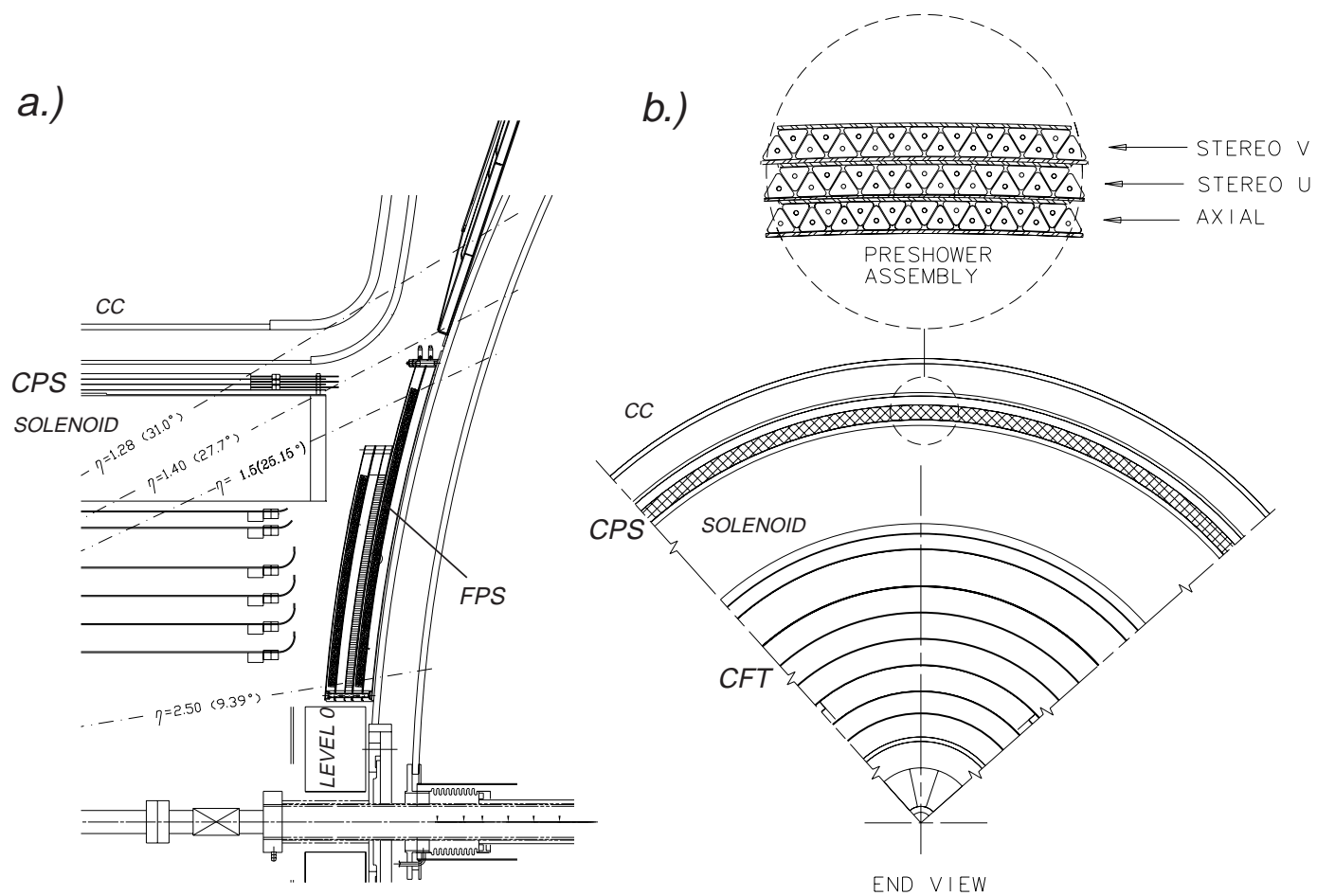

Figure 3.15: $x-y$ and $r-z$ views of the Central Preshower Detector.

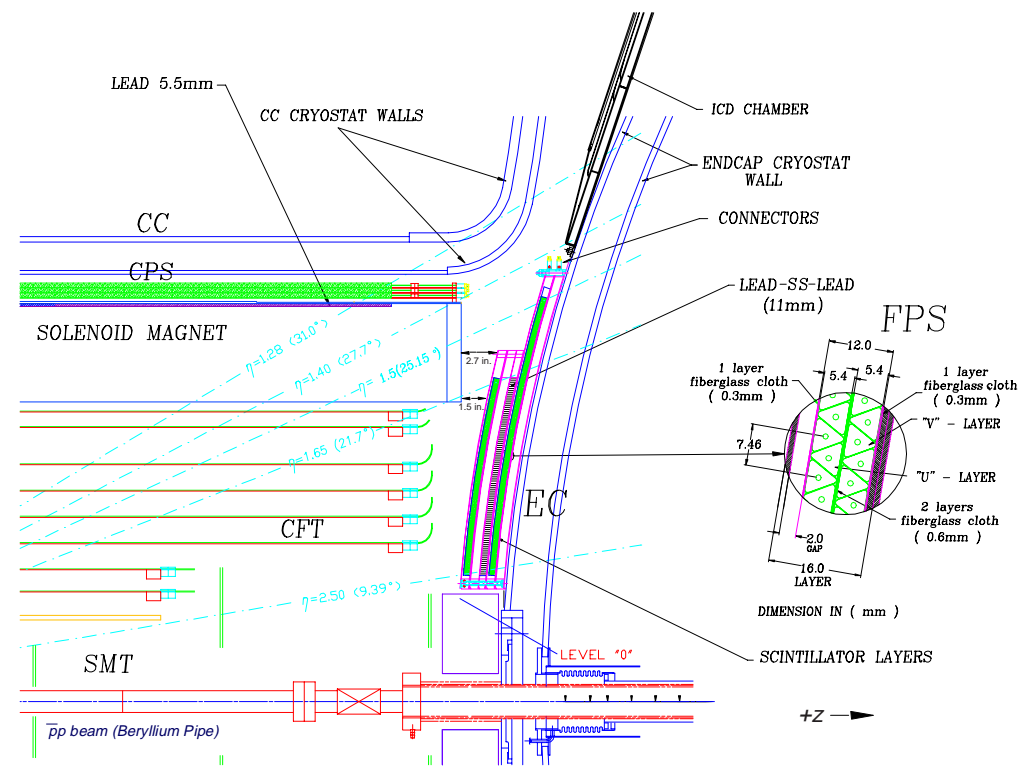

Figure 3.16: Location of Forward Preshower Detector in the DØ Detector 


\subsection{Luminosity Monitor}

The instantaneous luminosity monitoring is an important issue for any collider experiment, since precise cross section measurements rely completely on the known integrated luminosity of the data sample [6]. In the $h \rightarrow \gamma \gamma$ analysis integrated luminosity is used for setting limits on the Higgs production cross section.

The DØ Luminosity Monitor in Run II consists of two hodoscopes of scintillation pixels mounted on the face of the end cryostats [53]. The layout, location, and some relevant parameters [72] of the Luminosity Monitor are shown in Figure 3.17.

\section{Luminosity Monitor}

- Plastic scintillators with photomultipliers.

- 24 wedges mounted on each calorimeter end-cap at $\mathrm{z} \approx \pm 140 \mathrm{~cm}$.

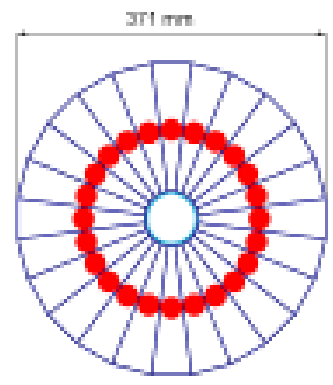

- Coverage is $2.7<|\eta|<4$.4. cc

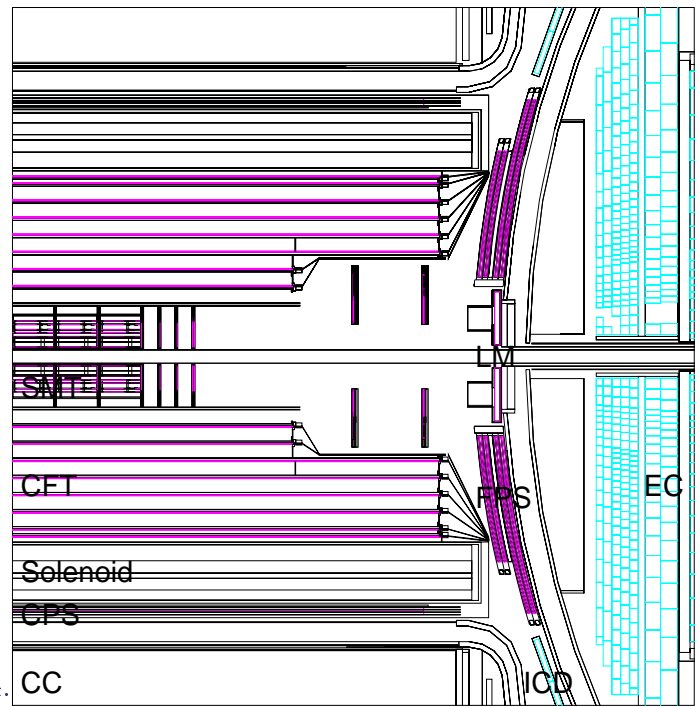

- Located in $\approx 1$ Tesla magnetic field.

- Time-of-flight resolution $\approx 200$ ps.

Figure 3.17: DØ Luminosity Monitor.

The scintillation light is read out using photomultiplier tubes. These counters detect nondiffractive $^{2}$ inelastic collisions with high efficiency. The rate of these collisions is used for determining the luminosity. In addition, the luminosity monitor is a tool that provides diagnostic information

\footnotetext{
${ }^{2}$ In diffractive $p \bar{p}$ scattering nucleon substructure is not revealed, i.e. it is a nucleon-nucleon scattering. Nondiffractive scattering one the other hand is a scattering in which either scattering participant is a nucleon constituent (a quark or a gluon).
} 
regarding the accelerator performance and also can be used to help indentify number of interactions per beam crossing. More information on the Luminosity Monitor can be found in Refs. [73, 74, 75].

\subsection{Muon System}

The goal of the muon system is identification of muons and independent measurement of their momenta in the toroidal magnetic field. In the $h \rightarrow \gamma \gamma$ analysis we do not use the muon system information, however, it is crucial for many physics topics covered by D, , including Higgs searches in the $W W$ and $b \bar{b}$ decay modes.

The muon system is the outermost detector system. Being much heavier than the electron, muons typically do not lose much energy via bremsstrahlung [6]. The energy loss mostly occurs due to ionization of the detector media which is a low energy-loss absorption process. Thus, muons with energy above a certain threshold $(\approx 3.5-5.0 \mathrm{GeV})$ pass through the entire DØ detector. Therefore the muon detector is located outside the calorimeter and is well protected from the debris from the hadronic and electromagnetic showers by the thick calorimeter material. The D $\varnothing$ muon system is shown in Figure 3.18 [46]. It has three major components [45]:

- Wide Angle MUon Spectrometer (WAMUS) covering $|\eta|<1$;

- Forward Angle MUon Spectrometer (FAMUS) covering $1<|\eta|<2$;

- Solid-iron magnet creating toroidal field of $1.8 \mathrm{~T}$.

The WAMUS has two types of detectors: proportional drift tubes (PDT) and scintillators. The tubes use the following gas mixture [53]: $80 \%$ argon, $10 \% \mathrm{CH}_{4}$, and $10 \% C F_{4}$. When operated at a voltage of $2.5 \mathrm{kV}$ for the pads and $5.0 \mathrm{kV}$ for the wires, the drift velocity in this gas is around $10 \mathrm{~cm} / \mu \mathrm{s}$, with a maximum drift time of $500 \mathrm{~ns}$. The timing resolution of the scintillators is $\sim 5 \mathrm{~ns}$. There are three WAMUS layers. The PDTs are located in all three layers, while the scintillators are arranged in two layers (no middle-layer scintillator).

The FAMUS has a similar structure: two types of detectors (Mini Drift Tubes (MDT) and scintillation pixels) are arranged in layers as shown in Figure 3.18. 


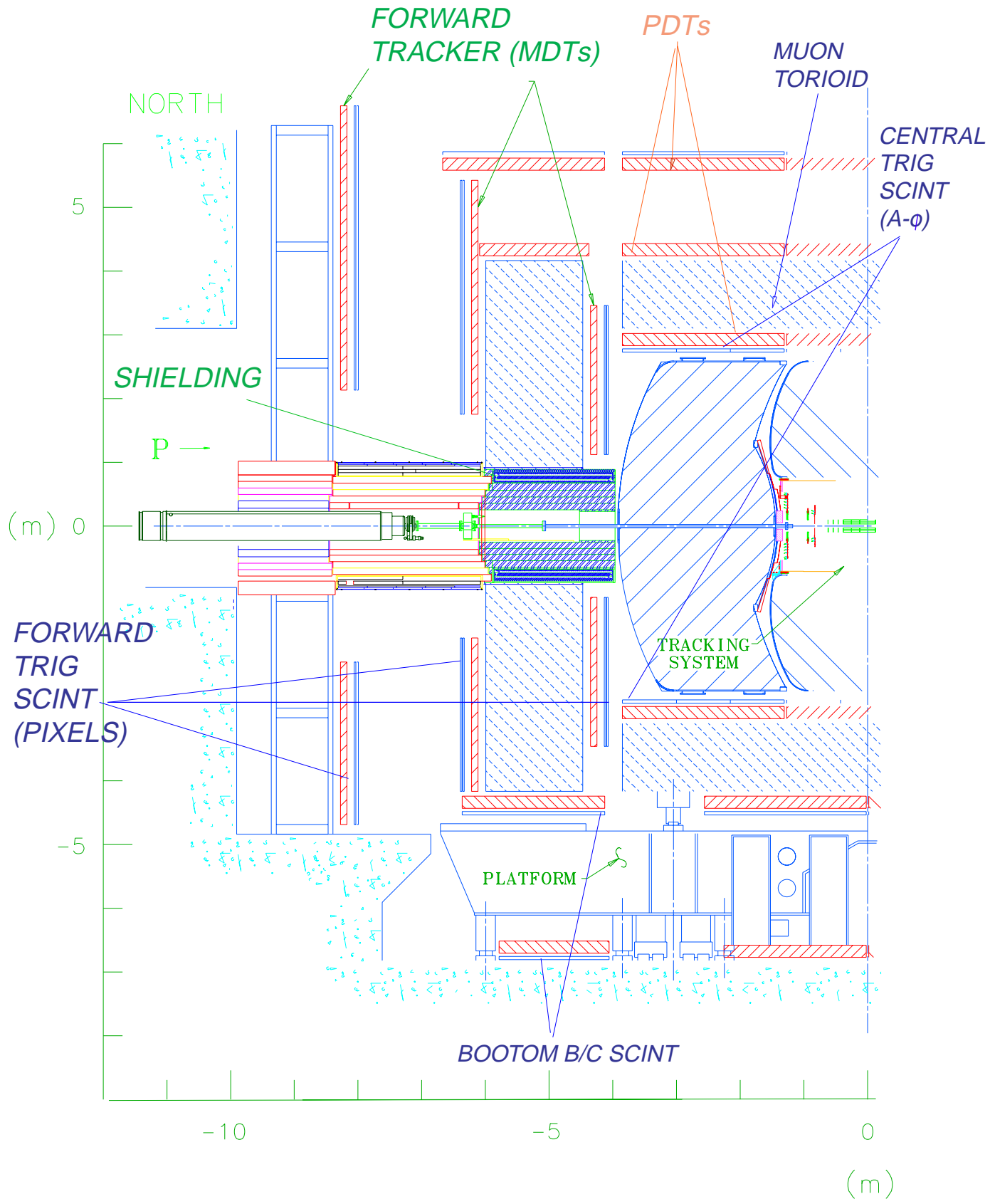

Figure 3.18: DØ muon detection system. 


\subsection{Forward Proton Detector}

The Forward Proton Detector (FPD) was designed to study non-perturbative QCD phenomena of elastic and diffractive $p \bar{p}$ scattering [76]. It consists of a series of spectrometers which are located on both sides of the $\mathrm{D} \varnothing$ interaction region, about $30 \mathrm{~m}$ away form the center of the detector. The spectrometer is a scintillating fiber detector placed in a stainless steel container (Roman pot) that allows the detector to function close to the beam. The pots have thin windows at the pot entrance and exit to allow protons to pass through. The pots are remotely controlled and can be moved close to the beam (within a few $\mathrm{mm}$ ) during stable beam conditions and retracted otherwise. The detectors measure the (x,y) position of a deflected proton (or anti-proton) track at the pot position. This information is used for measuring proton scattering angle and its momentum. For the momentum measurement magnetic field created by Tevatron magnets is used. The FPD has not been used in this analysis.

\subsection{Trigger and Data Acquisition Systems}

In a typical $p \bar{p}$ colliding-beam experiment roughly a few events in a million are of current physics interest, e.g. those in which $\mathrm{W}, \mathrm{Z}$, or top quark are produced. Higgs and other new phenomena events are expected to contribute even a smaller fraction. The rest of the events are due to low- $p_{T}$ non-diffractive $p \bar{p}$ scattering and parton scattering events. These processes have been thoroughly studied in the past, thus making these events less interesting for studies.

The total collision rate, however, exceeds by far the rate at which event processing and recording can be performed. Hence, most of the non-interesting events are discarded. For the events that are recorded, a proper balance between different physics processes based on the research priorities, rather than relative production rates, is required.

This is done by the means of triggers. Trigger is a device that looks at the coarse detector information in the event and quickly decides whether to keep or reject it, according to a specified pattern that corresponds to a particular type of event. For example, a basic trigger designed to select 
$p \bar{p} \rightarrow h \rightarrow \gamma \gamma$ events would look for two energetic EM towers above certain threshold (supposedly due to two photons) and reject events with only one EM tower as well as all-hadronic events.

The DØ trigger framework is organized into three main levels (L1, L2, L3) of increasingly sophisticated event selection and decreasing output rate [6]. The overall $\mathrm{D} \varnothing$ trigger scheme is shown in Figure 3.19 [46], which indicates the event rate at each stage. For example, the input rate into the

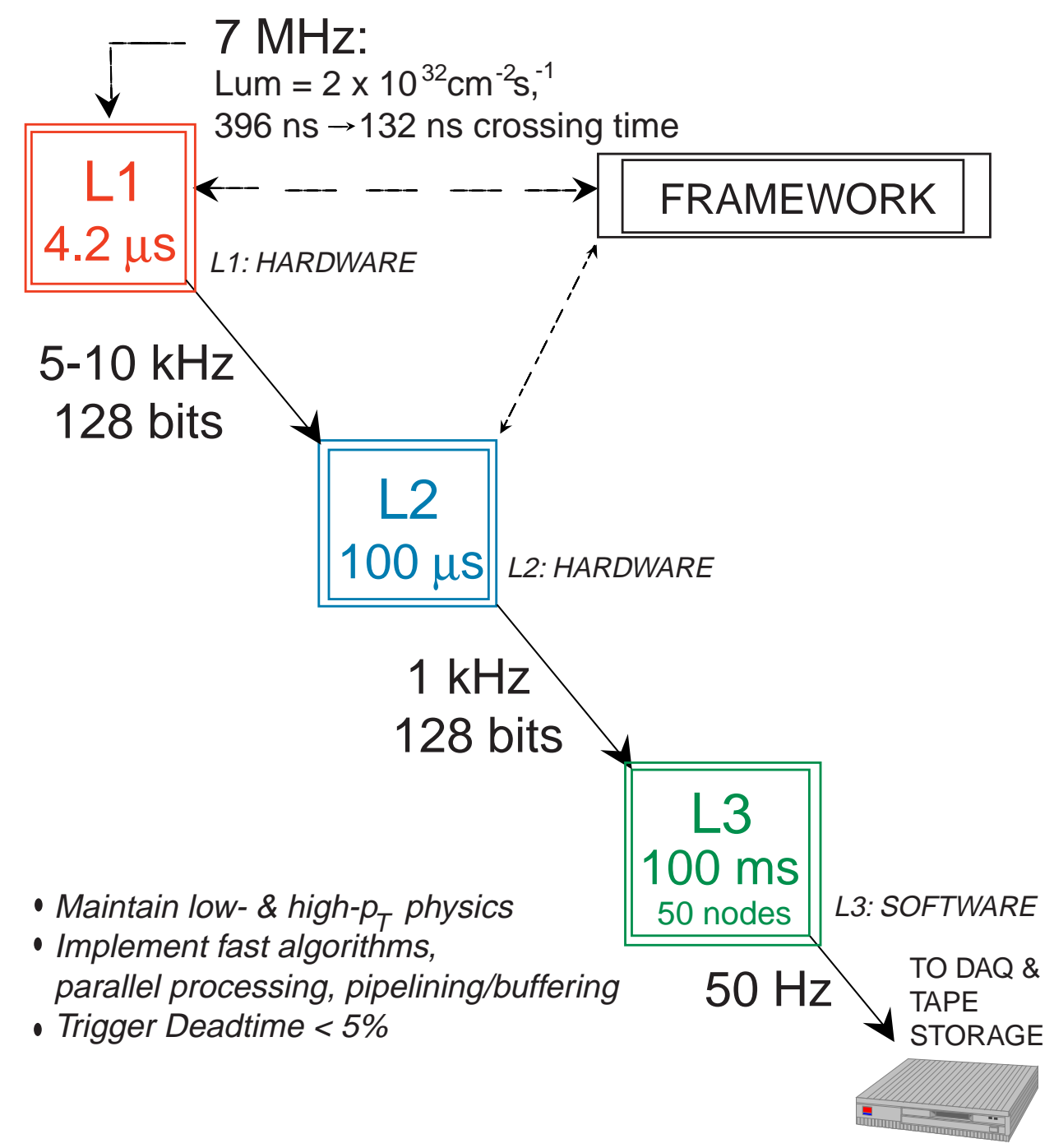

Figure 3.19: DØ trigger scheme layout and typical trigger rates.

trigger system (collision rate) is $2.5 \mathrm{MHz}$ while the $\mathrm{L} 3$ output rate is $\sim 50 \mathrm{~Hz}$. Thus, the reduction in the event rate due to the trigger selection is of the order of a million.

L1 is a hardware trigger system based on simple algorithms implemented in Field Programmable Gate Arrays (FPGAs) and the raw information from the scintillating fiber tracker (and preshower 
detectors), the calorimeters, and the muon system. At L1, information from each detector (or a group of detectors) is processed in parallel, as shown in Figure 3.20 [46].

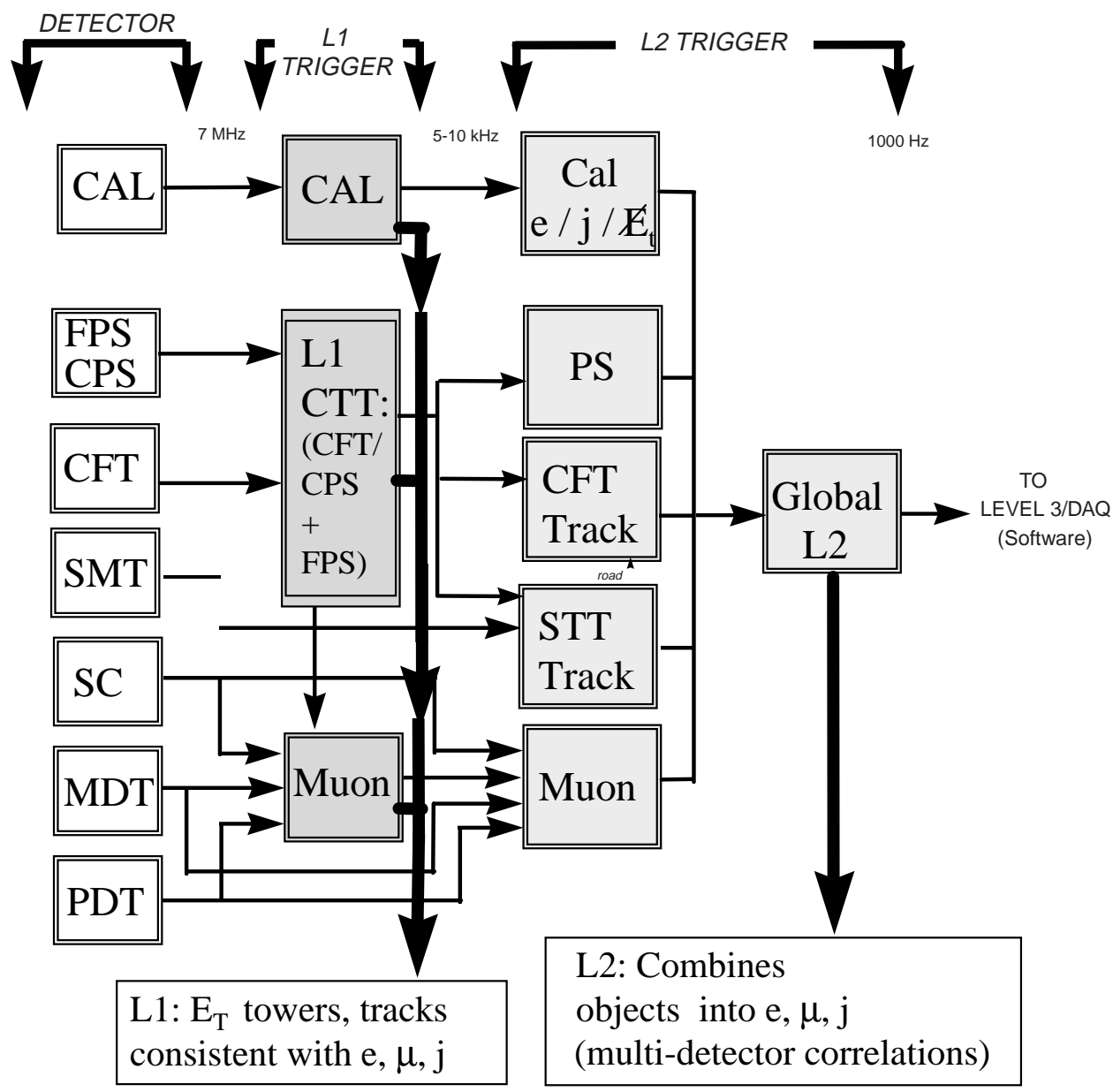

Figure 3.20: The L1 and L2 trigger data flow path.

The L2 trigger at its final stage correlates the information from different subdetectors as shown in Figure 3.21 [46]. Hardware-wise the basic L2 unit is a VME crate with $500 \mathrm{MHz}$ Alpha processors running Linux, VME bus, and a custom-built "Magic Bus" interfaces for 320Mb/s data handling $[77]$.

Events accepted by the L2 trigger are input to the L3/Data Acquisition System (DAQ). The Level 3 trigger combines and partially reconstructs full data for each event [78]. The L3 software runs on a farm of Linux PC's. Each event is analyzed by a different processor, which runs an independent instance of the L3 filtering software [47, 78, 79]. A diagram of the L3/DAQ system is shown in Figure 3.22 [79]. 


\section{Level 2 Trigger Block Diagram}

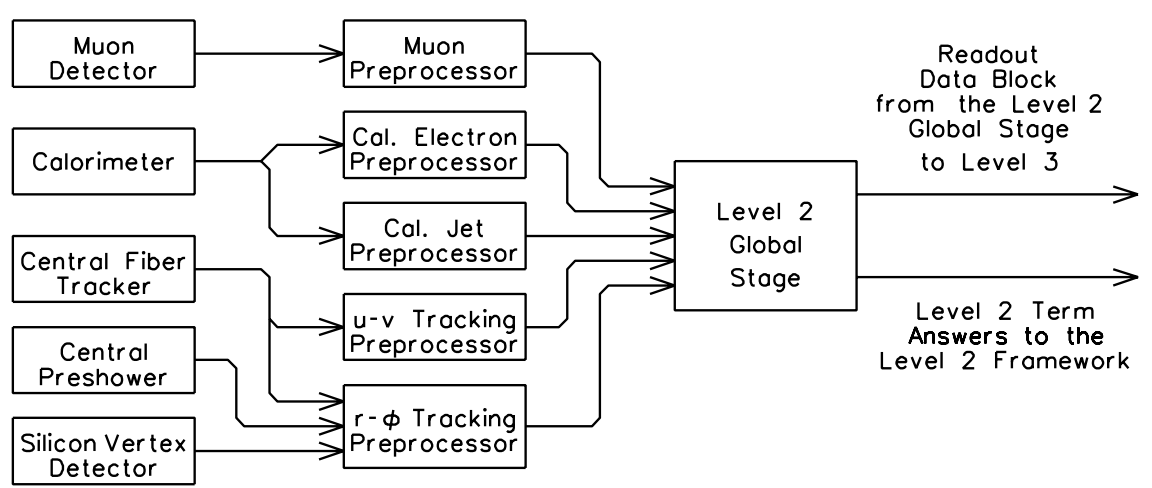

Figure 3.21: The L2 configuration.

\section{The L3DAQ System}

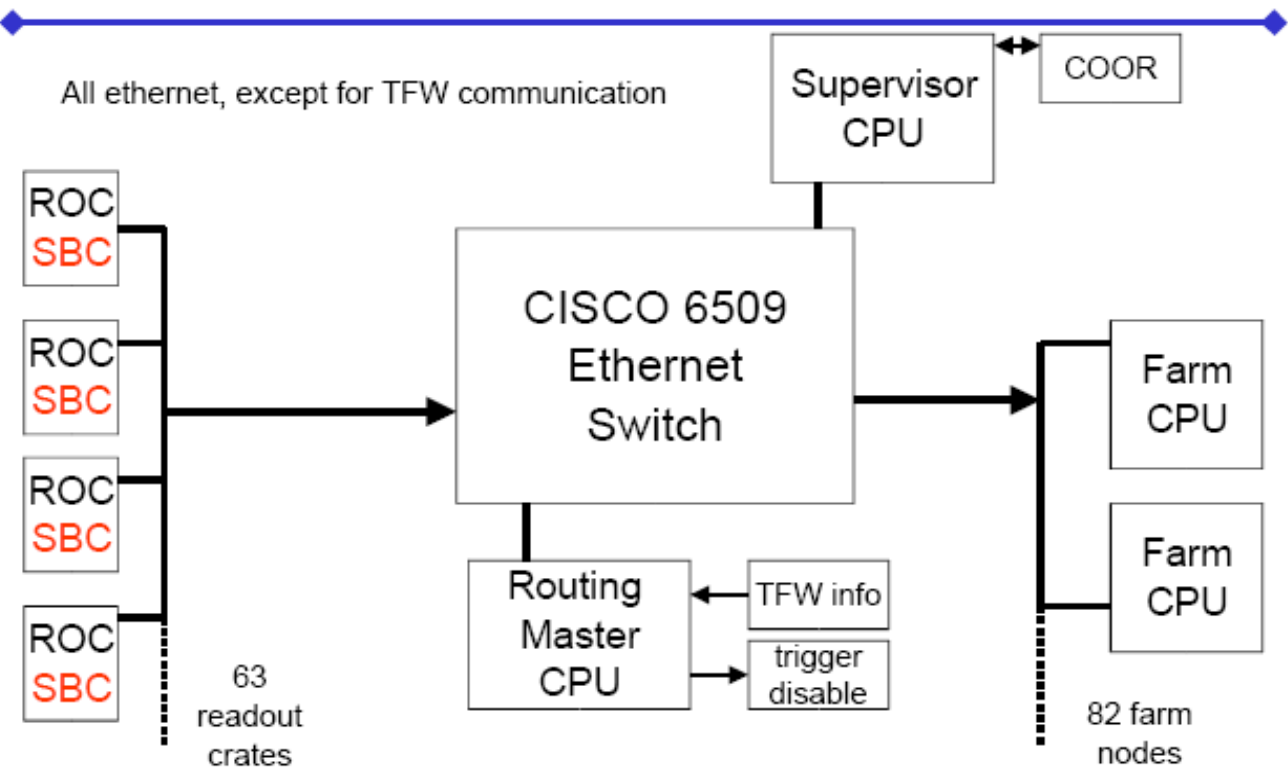

Figure 3.22: The L3/DAQ system layout. 
The data flow in parallel out of about $70 \mathrm{VME}$ readout crates (ROC), each corresponding to a section of a subdetector system or the trigger framework. Each crate is read out by a Single Board Computer (SBC). An SBC is powered by a $933 \mathrm{MHz}$ Pentium-III processor with $128 \mathrm{MB}$ of RAM. Data size in each crate is $1-10 \mathrm{kB}$. The total event size is about $250 \mathrm{kB}$. The data are moved out of SBCs over the Ethernet via a series of Ethernet connections, which transfer them over to the main switch via $\mathrm{a} 1 \mathrm{~Gb} / \mathrm{s}$ optical fiber. The farm nodes receive data fragments through the main switch. A farm node builds a complete event, reconstructs it, and performs physics selection. Finally, events that pass the physics criteria are sent via the network to a collector machine and are eventually written to tape for offline analysis. This is a simplified picture, which leaves out the devices that ensure coordination of the L3/DAQ components both functionally and in terms of time synchronization. 


\title{
Chapter 4
}

\section{Photon Identification}

\author{
with brightest light \\ that passes far to all the sides... \\ ...bored eye shall not then find \\ familiar faces in the show \\ Alexander Pushkin, "Evgeniy Onegin"
}

The goals of Photon Identification are:

- recognize patterns in the detector signals that are likely to have been produced by a photon;

- estimate photon reconstruction and identification efficiency;

- estimate the probability of photon misidentification.

Experimental signature of a photon at a multi-system collider detector is:

1. A shower in the calorimeter. The shape of the shower must be consistent with that for an electromagnetic shower.

2. No tracks that can be associated with the shower should be found by the tracking system.

Before discussing the methods of photon identification, let us discuss the properties and underlying processes in the electromagnetic and hadronic showers. 


\subsection{Electromagnetic Showers}

Relevant electromagnetic interactions in the photon or electron-induced showers are as follows:

- Bremsstrahlung (photon emission by an electron decelerated in a field of the atom);

- Electron-positron pair production $\left(\gamma \rightarrow e^{+} e^{-}\right)$;

- Compton scattering (photon-electron scattering);

- Coulomb scattering (electron-nucleon scattering);

- Bhabha scattering (electron-positron scattering);

- Möller scattering (electron-electron scattering);

- Photoelectric effect (electron emission from the $\gamma$-irradiated atoms)

- Annihilation $\left(e^{+} e^{-} \rightarrow \mu^{+} \mu^{-}\right.$, or $\left.\rightarrow \gamma\right)$.

Most of the above processes occur only at low energies. High energy electrons (energies above $100 \mathrm{MeV}$ ) predominantly loose their energy via bremsstrahlung, while the high energy photons via $e^{+} e^{-}$pair production. Energy loss as a function of incident particle's energy is shown in Figs. 4.1 and 4.2 for electrons and photons, respectively. [22]

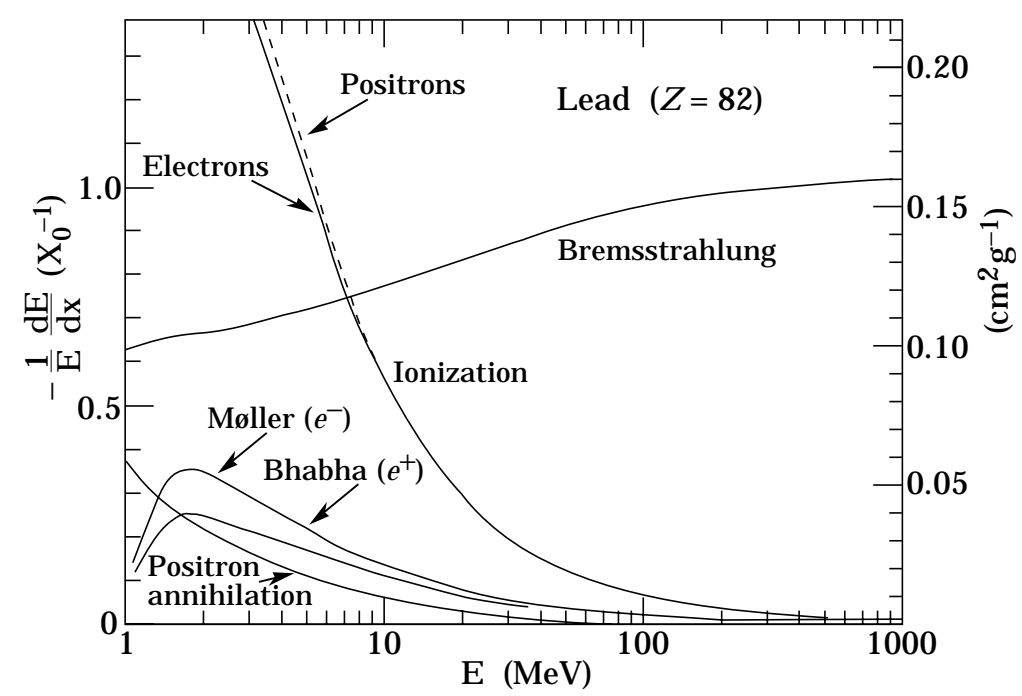

Figure 4.1: Fractional energy loss per radiation length in lead as a function of the electron or positron energy. At high energies bremsstrahlung dominates. 


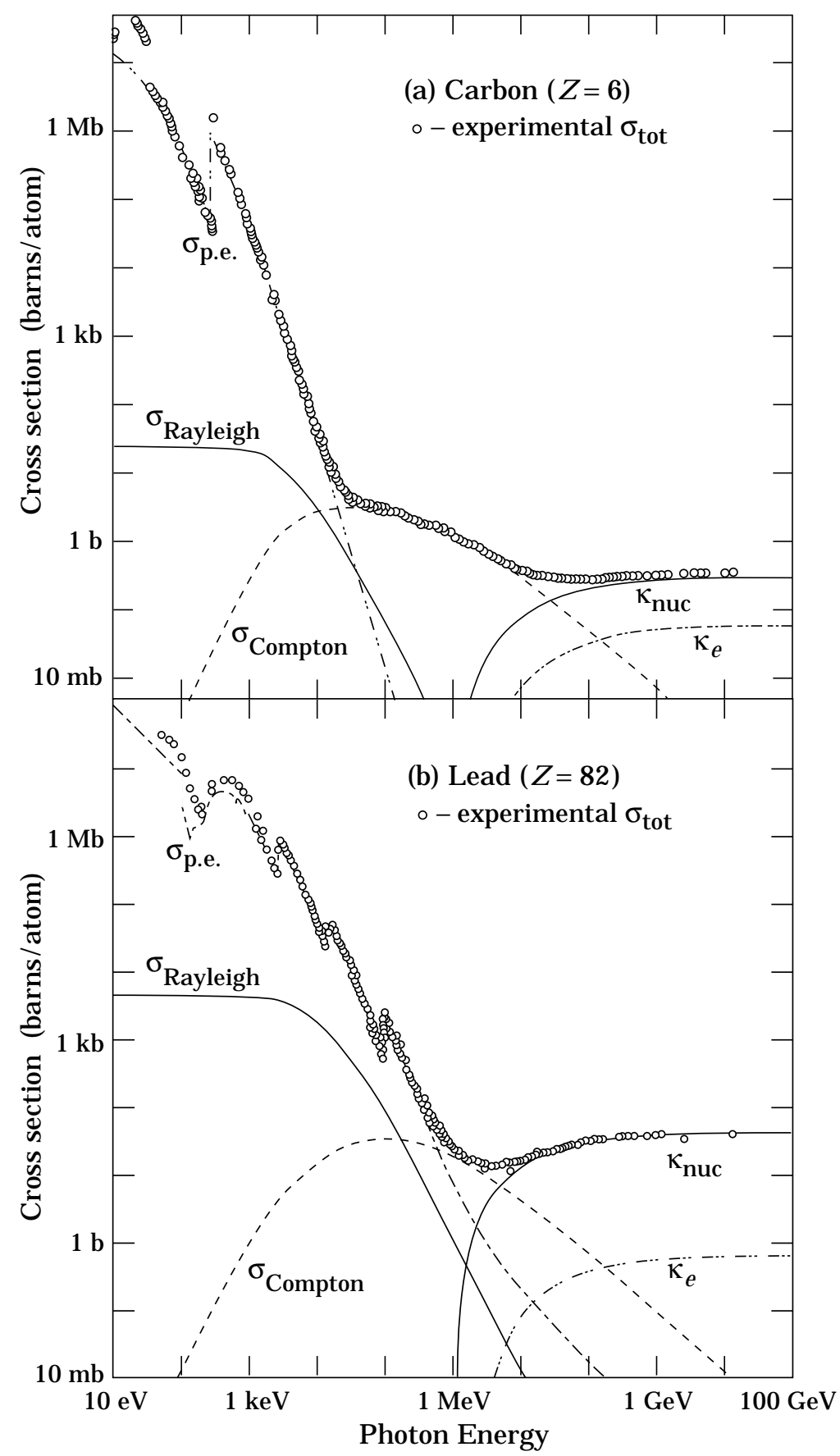

Figure 4.2: Total photon cross sections as a function of energy in carbon and lead, showing contributions of different processes. At high energies pair production dominates $\left(\mathcal{K}_{n u c}\right.$ and $\mathcal{K}_{e}$ denote pair production in nuclear and electron fields, respectively). 
The development of a shower can be conveniently expressed in terms of a scaling variable (i.e. a variable that makes the description independent of the material used), the radiation length, usually denoted by $X_{0}$. It characterizes the energy loss for the electrons in the material [60] and is defined by the relation;

$$
E(\ell)=E_{0} \exp \left(-\ell / X_{0}\right)
$$

where $E_{0}$ is the initial electron energy and $E(\ell)$ the average energy after passing through thickness $\ell$ of the material. The $X_{0}$ has a two-fold meaning. In addition to being an appropriate electromagnetic shower length scale, it corresponds to the thickness of the material in which individual electron's energy decreases by a factor of $1 / e$ on average. The energy loss of a high energy photon by pair production is directly related to $X_{0}$. A high energy $\gamma$ will produce and electron-positron pair in a thickness $X_{0}$ of the material with probability $7 / 9$.

In general, $X_{0}$ is a function of electron energy and the nature of the absorber material. To a first approximation, the radiation length decreases as $Z^{-2}(Z$ is the atomic number of the absorber), consequently, calorimeters generally use high- $Z$ materials (such as uranium in the case of $D \emptyset$ ) in order to minimize their overall size.

To summarize, the electromagnetic shower develops via multiplication of electrons and photons. Electrons (and positrons) are created by pair production process. Photons are created in bremsstrahlung. The energy of initial incident electron or photon is shared between particles in the shower and dissipates as they travel through the detector material. Particles in a shower continue to multiply until a maximum number of particles is reached when the average particle energy is no longer high enough to continue the multiplication process. Then other interaction processes start contributing to the development of the shower and eventually all energy is absorbed by the detector material. The crossover point at which electron loses equal amounts of energy via bremsstrahlung and ionization is called critical energy $\epsilon$. 


\subsection{Hadronic Showers}

With a much wider range of physics phenomena involved in the development of a hadronic shower its description either in approximate form, or in detail, is not nearly as advanced as that for electromagnetic showers [59]. Let us discuss briefly the contributing processes. The first possibility is that a particle interacts with an atomic nucleus [61]. For hadrons the short-range interactions with the nuclei via the strong force are most important. The total nuclear cross-section can be divided into three parts :

$$
\sigma_{t o t}=\sigma_{e l}+\sigma_{q}+\sigma_{\text {inel }} .
$$

The first term, $\sigma_{e l}$, refers to the elastic scattering from the nucleus. The second, $\sigma_{q}$, is the quasielastic scattering (i.e. elastic scattering from a constituent nucleon, in which nucleon recoil leads to nuclear excitation or break-up). In elastic and quasi-elastic scattering a hadron retains its identity and its momentum in general is only slightly perturbed. Therefore these two processes to not contribute significantly to hadron energy dissipation in the hadronic shower. The final contribution $\sigma_{\text {inel }}$ refers to inelastic scattering from the constituent nucleons, and gives the biggest contribution at high energies. This type of interaction is mainly responsible for the shower attenuation. The probability of a inelastic hadron-nucleus interaction occurring as the hadron traverses a small thickness $d x$ of the material is given by $n \sigma_{\text {inel }} d x$, where $n$ is the number of nuclei per unit volume in the material. Consequently, the mean distance traveled before an inelastic interaction occurs is given by

$$
\lambda \approx \frac{1}{n \sigma_{\text {inel }}}
$$

$\lambda$ is called the absorption length. This quantity is analogous to the radiation length $X_{0}$ in case of electromagnetic showers. It defines the distance scale of a hadronic shower. A hadronic shower typically develops as follows. About half of the incident hadron energy is consumed in a multiparticle production (slow pions of typical transverse momentum of $0.35 \mathrm{GeV}$ ) and other processes [62, 63].

The remainder of the energy is carried by fast forward-going secondaries. The secondaries are mostly pions and nucleons, and their multiplicity depends on energy only weakly [63, 64]. If a fluctuation 
happens in such a way that most of the hadronic shower energy is carried by a secondary $\pi^{0}$ (or other neutral mesons with large branching fractions to photons such as $\eta$ ) the shower would look very much like the electromagnetic one since $\pi^{0}$ decays to two photons with $98 \%$ branching fraction The opening angle $\theta$ between the two photons is given by the following expression [61]:

$$
\cos \theta=1-m_{\pi^{0}} / 2 E_{1} E_{2}
$$

where $m_{\pi^{0}}=138.98 \mathrm{MeV}$ is the $\pi^{0}$ mass; $E_{1}$ and $E_{2}$ are photon energies. This expression suggests that for the photon energies of the order of a few $\mathrm{GeV}$ the opening angle is very small. Therefore such a shower would be difficult to distinguish from the one generated by a single final state photon (for example, coming from the Higgs decay). This is the major source of background for the photon identification.

\subsection{Electromagnetic and Hadronic Shower Shape}

For the purposes of Photon Identification the following shower properties need to be understood:

- longitudinal shower extent and energy loss profile along the length;

- transverse shower profile;

These properties can be described in terms of radiation length $X_{0}$ and absorption length $\lambda$ for the electromagnetic and hadronic showers, respectively. Table 4.1 shows $X_{0}$ and $\lambda$ for different materials [60], along with the critical energy $\epsilon$.

\begin{tabular}{cccc}
\hline material & $X_{0}(\mathrm{~cm})$ & $\lambda(\mathrm{cm})$ & $\epsilon(\mathrm{MeV})$ \\
\hline NaI & 2.6 & 41 & 12.5 \\
Liquid Argon & 14.0 & 81 & 29.8 \\
$\mathrm{Fe}$ & 1.76 & 17 & 20.5 \\
$\mathrm{~Pb}$ & 0.56 & 18 & 7.2 \\
$\mathrm{U}$ & 0.32 & 12 & 6.6 \\
\hline
\end{tabular}

Table 4.1: Radiation length $X_{0}$, absorption length $\lambda$, and critical energy $\epsilon$ for several materials. 


\subsubsection{Longitudinal Extent and Energy Loss}

The longitudinal depth of the electromagnetic shower induced by an incident particle of energy $\mathrm{E}$ is given by

$$
L=t_{\max }+x .
$$

The first term $t_{\max }$ corresponds to the depth at which maximum energy is deposited (shower maximum):

$$
t_{\max }=X_{0}[\log (E / \epsilon)-1] .
$$

The second term $x$ measures the attenuation phase of the shower, at which energy deposition decreases exponentially with the depth until no measurable signal is produced. An example of a longitudinal electromagnetic shower profile is shown in Figure 4.3 [22, 65]. Full longitudinal ex-

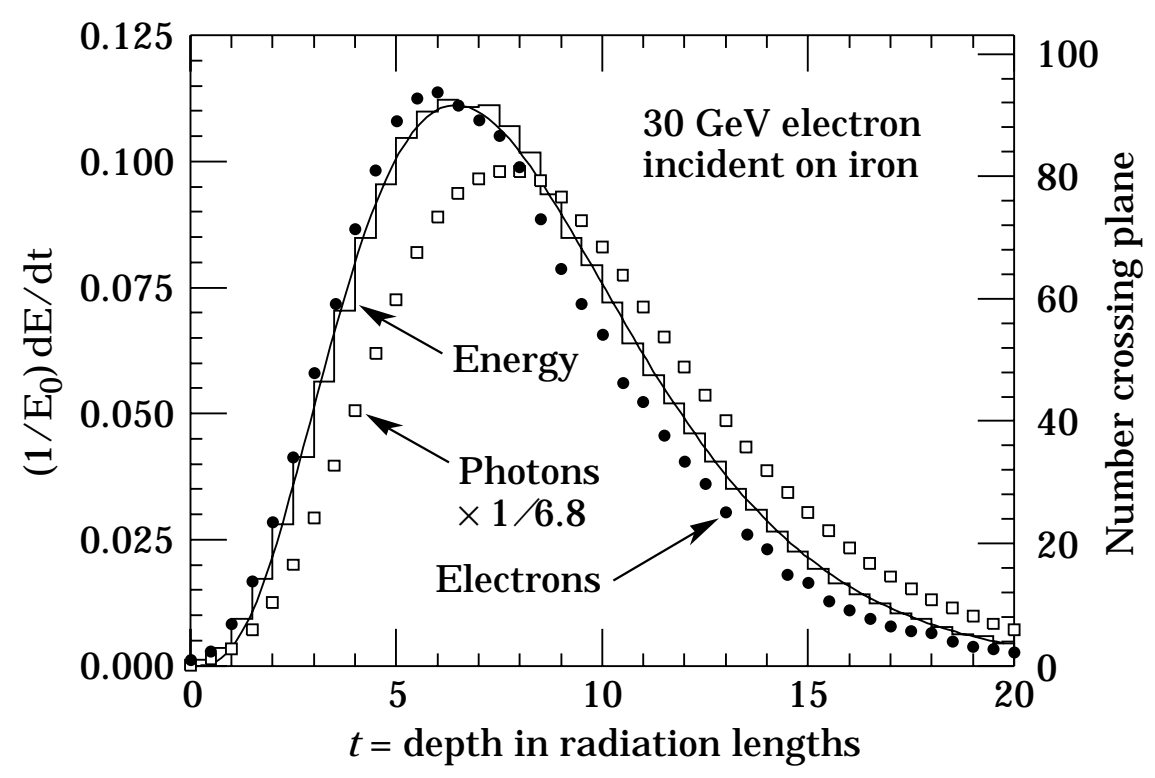

Figure 4.3: $30 \mathrm{GeV}$ electron cascade induced in iron (EGS4 simulation). The histogram shows fractional energy deposition per radiation length, and the curve is a gamma-function fit to the distribution. Circles indicate the number of electrons with total energy greater than $1.5 \mathrm{MeV}$ crossing planes at $X_{0} / 2$ intervals (scale on the right) and the squares are the number of photons with $E \geq$ $1.5 \mathrm{MeV}$ crossing the planes (scaled down to have the same area as the electron distribution).

tent of a shower corresponds to $\sim 20 X_{0}$, which defines the optimal thickness of electromagnetic calorimeters (e.g., the depth of the DØ Central EM calorimeter is $21 X_{0}$, see Table 3.1 in Section $3.6 .2)$. 
Longitudinal energy deposition profiles for hadronic showers are characterized by a sharp peak near the first interaction point (from the fairly local deposition of EM energy resulting from the $\pi^{0}$ 's produced in the first interaction), followed by a more gradual development $[22,66,67]$ with a maximum at

$$
t_{\text {max }}=\lambda[0.2 \log (E / 1 \mathrm{GeV})+0.7]
$$

measured from the face of the calorimeter The longitudinal dimension required for $95 \%$ containment of the shower energy is approximated by

$$
L_{0.95}=t_{\max }+2.5 \lambda_{a t t}
$$

The quantity $\lambda_{\text {att }}$ describes the exponential decay of the shower beyond $t_{\max }$ and increases with the energy approximately as $\lambda_{a t t} \approx \lambda[E(G e V)]^{0.13}$.

\subsubsection{Transverse Shower Shape}

The transverse spread of the electromagnetic shower is determined by the multiple scattering of the electrons. Bremsstrahlung and pair production processes at high energies are predominantly very forward-peaked and do not contribute significantly to the shower spread. The transverse spread can be expressed in terms of the Moliere radius, $R_{M}$, the parameter of the multiple scattering theory defined as

$$
R_{M}=21 \mathrm{MeV} \frac{X_{0}}{\epsilon}
$$

About $90 \%$ of the shower energy is contained within $R_{M}$, which is $\sim 3 X_{0}$ in case of $\mathrm{Pb}$. The transverse radius of the $95 \%$ containment of the hadronic shower is very approximately

$$
R_{0.95} \leq \lambda
$$

Earlier in this section we mentioned the expressions describing the geometrical parameters of the electromagnetic and hadronic showers in terms of scaling variables $X_{0}$ and $\lambda$. Since $X_{0}$ is generally 
at least by an order of magnitude smaller than $\lambda$ (Table 4.1) the shower created by a photon or an electron is expected to be smaller in size than the hadronic shower both longitudinally and transversely.

\subsection{Cluster Reconstruction and EMID variables}

Cluster-finding algorithm searches through the list of calorimeter cells with significant energy deposits to find clusters of adjacent cells. Then the shape and the energy profile of the cluster needs to be verified in order to accept it as a photon (or electron) candidate. This is performed in several steps by the means of $\mathrm{EMID}^{1}$ variables.

The cluster has to pass crude initial selection criteria imposed by the $\mathrm{D} \varnothing$ reconstruction program [80]. Unless the ratio of the EM energy to the total energy in the cluster is above 0.9 and $p_{T}$ of the cluster $^{2}$ is above $1.5 \mathrm{GeV}$ the cluster is rejected. Then the Isolation (iso) variable is computed as follows ${ }^{3}$ :

- Make a list of towers within a Large Window contour in the $\eta-\phi$ space. The contour is centered on the tower with the highest $p_{T}$. This is illustrated [82] in Figure 4.4 .

- From this list of towers select those that lie within a $\operatorname{circle}^{4}$ of radius 0.4 in the $\eta-\phi$ space around the center of gravity of the cluster; compute the EisoTot variable = total energy in the selected towers through the full depth (EM and hadronic layers).

- Similarly select a 0.2 circle and compute the EisoCore variable = energy deposited in the EM layers of the calorimeter.

- Calculate the Isolation $($ iso $)$ variable : iso $=($ EisoTot - EisoCore $) /$ EisoCore. Figure 4.6 is a graphical representation of EisoTot, EisoCore and iso variables.

\footnotetext{
${ }^{1}$ EMID stands for electromagnetic identification, i.e. identification of photons and electrons

${ }^{3} p_{T}$ here means the same as $E_{T}=E \sin \theta$ (photon mass is zero and electron and quark masses are negligible for the energy range of our interest, above $20 \mathrm{GeV}$ ), where $E$ is the energy of the clusters measured by the calorimeter and angle $\theta$ is computed using the calorimeter cluster center of gravity position in the EM3 layer.

${ }^{3}$ this discussion is specific to the isolated central EM clusters. It does not apply to EM cluster reconstruction within a hadronic jet, nor to a cluster in the End Calorimeter.

${ }^{4}$ a circle of radius $\mathrm{R}$ in the context of discrete calorimeter geometry means a contour in the $\eta-\phi$ space within which all the towers whose centers are separated from the center of the circle by less than $\mathrm{R}$ lie. This is illustrated in Figure 4.5 .
} 


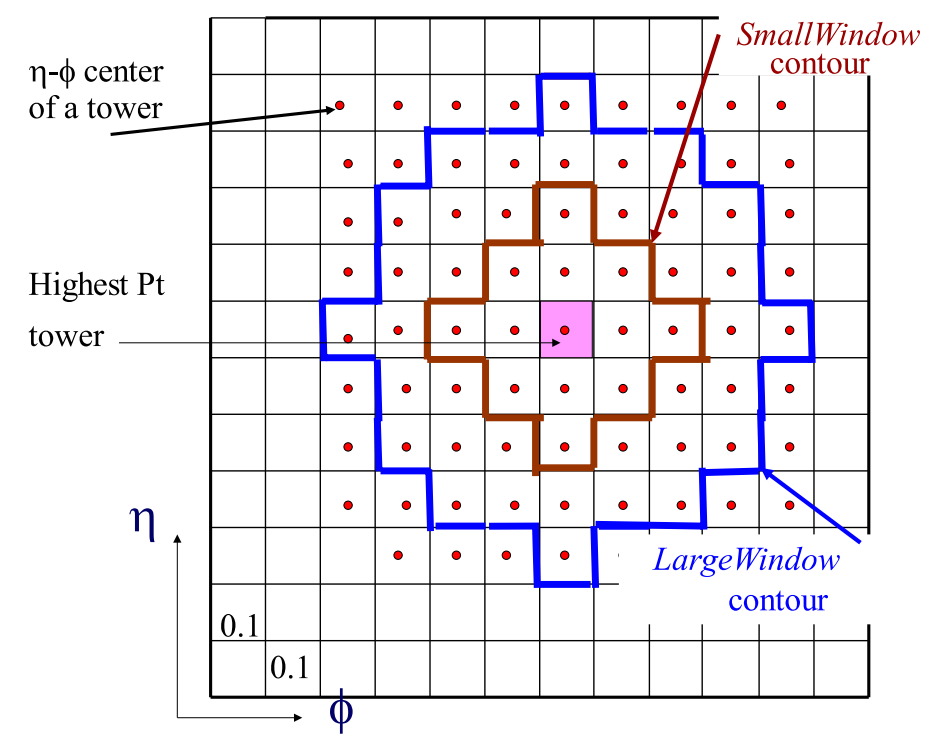

Figure 4.4: Cluster reconstruction $\eta-\phi$ window contours.

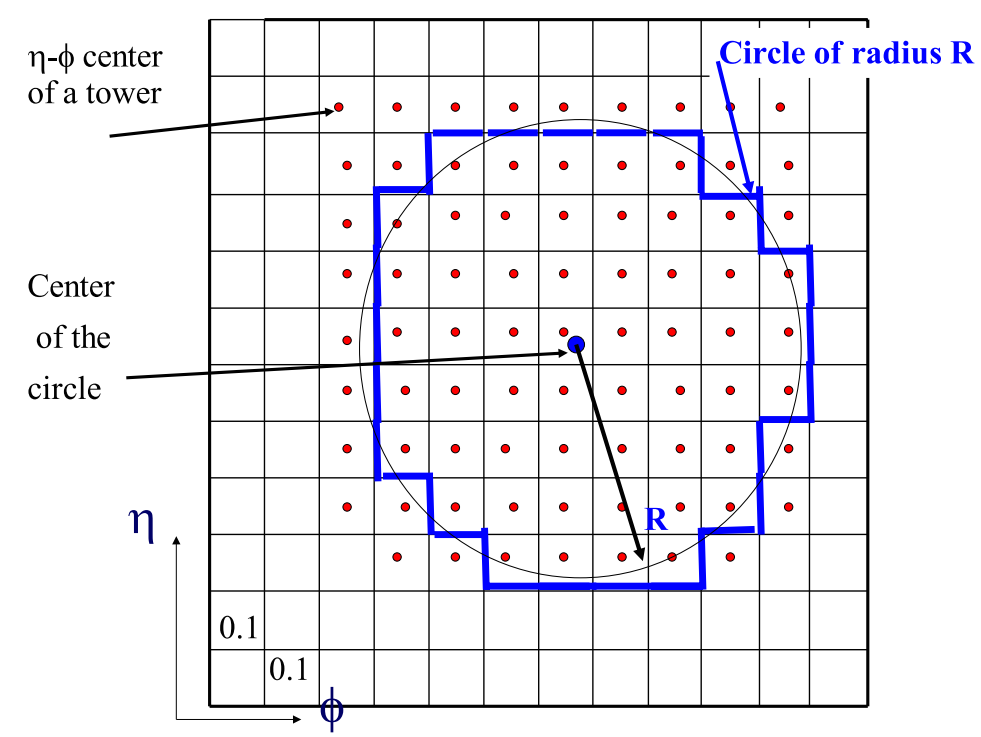

Figure 4.5: Cluster reconstruction $\eta-\phi$ circle contour. 


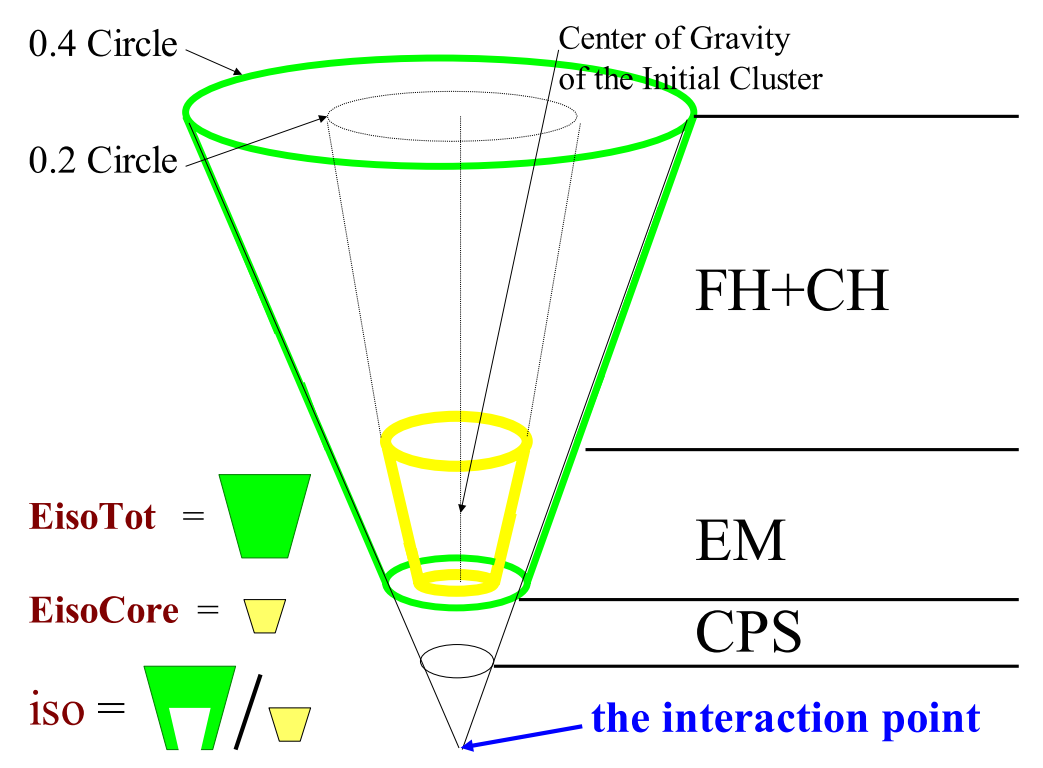

Figure 4.6: pictorial representation of the EisoTot,EisoCore and iso EMID varialbes.

The Isolation variable is a measure of how deep and narrow a cluster is. Small values of iso correspond to the situation when most of the energy is deposited in the narrow region of the EM calorimeter. Such deposition is likely to be originated by a photon or electron. The default iso threshold used at the reconstruction level is 0.2 . Note that this is a very loose cut because :

1. The longitudinal extent of the EM calorimeter was designed to fully contain electromagnetic showers (see Sections 3.6.2 and 4.3.1).

2. individual towers were designed for full transverse containment of electromagnetic showers. $0.1 \times 0.1$ square in the $\eta-\phi$ space contains a circle of the Moliere radius [96] (see Section 4.3.2).

During the analysis stage we tighten the iso cut to 0.15 (see Section 4.5). This is the optimal cut established by comparing EMID efficiency and hadronic background rejection [81].

A cluster that passes the default $i s o$ threshold enters the final stage of the reconstruction: final cluster building. At this stage various single variable quantities that describe cluster properties are computed and stored. A few examples such as Cluster Energy $(E)$, Hadronic Energy of a cluster $(H A)$, EM energy fraction (EM frac) are described pictorially in Figure 4.7. The Iso and EM frac are the two variables that are most useful for EM identification. In addition, a multi-variable tool 


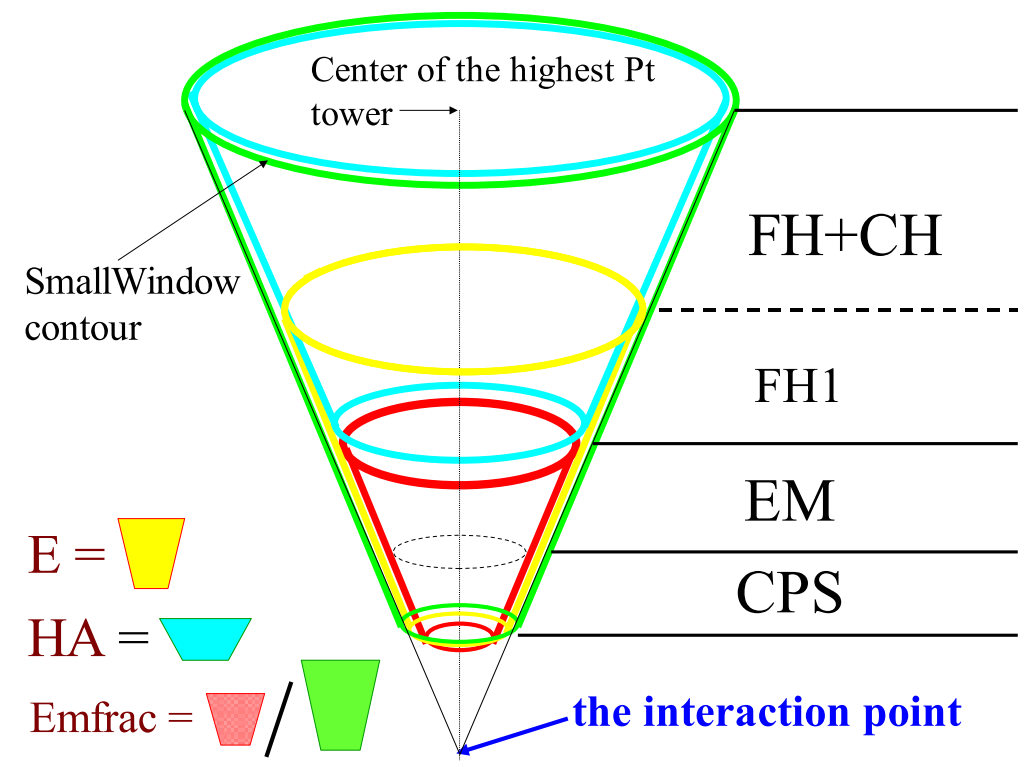

Figure 4.7: pictorial representation of cluster energy $(E)$, hadronic energy of a cluster $(H A)$, and EM energy fraction (EM frac) EMID varialbes

(H-matrix) is used to analyze the detailed shower shape of a cluster. This tool allows for a more reliable discrimination between the EM clusters and hadronic jets. It is the inverse of the covariance matrix built on the following variables [83]:

- fractions of the energy deposited in each of the four EM1, EM2, EM3, EM4 layers;

- cluster width in $r \phi$ and $z$ (energy weighted RMS computed with the EM3 cells);

- $\log _{10}($ ClusterEnergy);

- reconstructed interaction point position.

The H-Matrix is trained on the Monte Carlo sample of electrons (separate H-matrix is made for each $\eta$ bin). Then for each reconstructed cluster a $\chi^{2}$-like function ${ }^{5}$ is calculated using the H-matrix as an error matrix [6]. This $\chi^{2}$-like function is therefore a quantitative measure of the probability that the cluster is due to a single electron/photon. Monte-Carlo-simulated example distributions of the Isolation (iso), EM Fraction (EM frac), and H-Matrix $\chi^{2}$ EMID variables are shown in Figure 4.8 .

\footnotetext{
${ }^{5}$ The H-Matrix variables, in general, are not normally distributed and thus this function does not follow the $\chi^{2}$ distribution [12] exactly.
} 

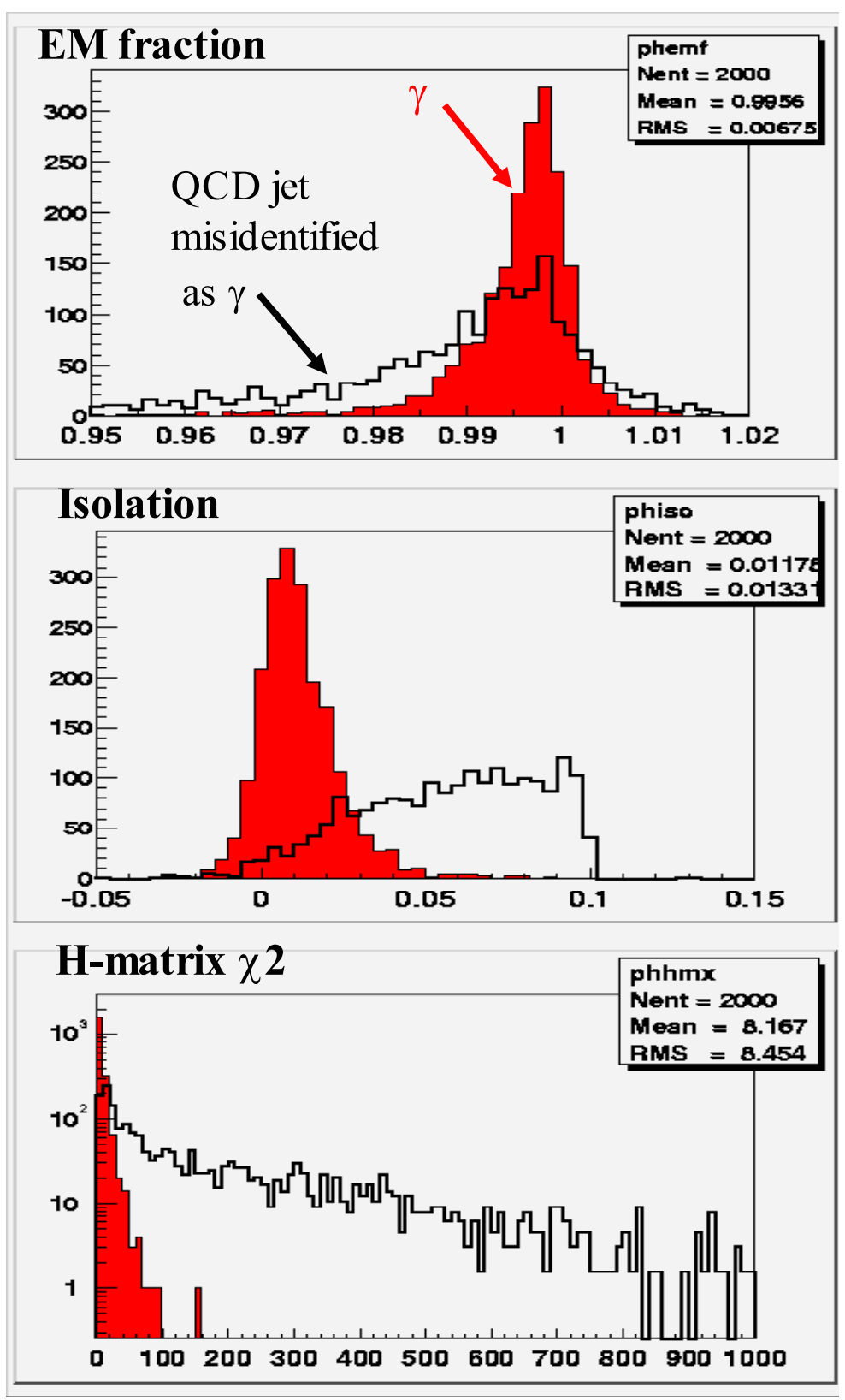

Figure 4.8: Monte-Carlo example of the distributions of Isolation (iso), EMFraction (EM frac), and $H-M a t r i x \chi^{2}$ EMID variables. Red: Single Photons; Black: Hadronic jets that passed iso $<0.1$ and $E M$ frac $>0.9$ (Probability for a hadronic jet to pass these cuts is $\approx 0.01$ ). In this example a tighter Isolation cut of 0.1 was used. In real data the discrimination is expected to be somewhat worse since the H-Matrix was trained on the Monte-Carlo sample that does not reproduce the data exactly. 


\subsection{Analysis Object Definitions}

The reconstructed cluster information is stored in the form of the $\mathrm{C}++$ objects [84] included in the full event information. At the analysis stage, these objects are further refined (energy scale and other corrections are applied, some variables are recomputed, various checks are performed, track matching is done) by the EMID software programs [85]. Final information about the electromagnetic clusters found in the calorimeter is then stored in objects of EMcandidate class. Out of EMcandidate objects we construct our analysis objects by applying the following EMID (Section 4.4) and kinematic cuts:

- Loose EM object is valid EMcandidate in the good $\eta$ fiducial region $(|\eta|<1.05,1.5<|\eta|<2.4)$ with $p_{T}>25 \mathrm{GeV}$ satisfying relatively loose EMID cuts: EM fraction $>0.9$, Isolation $<0.15$, HMatrix(EMshowershape) $\chi^{2}<40$;

- EM object is Loose EM object that passes HMatrix(EM showershape) $\chi^{2}<15$ cut;

- Electron is EM object that has a track match;

- Photon is EM object that has no track match;

To avoid possible ambiguity in the meaning of words 'Photon' and 'Electron' roman style will be used for particles and italics for Photon and Electron analysis objects. In those cases where such distinction is not needed, symbols ' $\gamma$ ' and 'e' will be used instead.

\subsection{Misidentification Rate}

Important aspect of the $h \rightarrow \gamma \gamma$ analysis is understanding of the Photon and Electron misidentification rates. In this section we shall focus on the following issues:

- What is meant by a 'Fake Photon/Electron';

- How is Photon/Electron fake rate defined;

- How to select a sample to measure Photon/Electron fake rate ;

- Results. 


\subsubsection{What is a 'Fake Photon'?}

A Fake Photon/Electron is a Photon/Electron found in the detector from a QCD event multijet event. QCD events produce Photons/Electrons in the detector dominantly due to :

(i) Final state Photons in direct diphoton or $\gamma+$ jet events;

(ii) Diphoton decays of energetic neutral mesons that carry significant fraction of jet energy in a multijet or $\gamma+$ jet events;

(iii) Fluctuations in measured jet energy and shape in a multijet or $\gamma+$ jet events, resulting in jets misidentified as Photons/Electrons.

In case of Electron, a matched track from a charged hadron in a jet or a fake track must be present additionally.

\subsubsection{Definition of Photon/Electron Fake Rate}

For the discussion of fake rates we shall concentrate on Photons and mention Electrons only at the end of this section when presenting quantitative results. Fake rate is a quantity (a number or a scalar function of $p_{T}$ ) which allows to predict QCD contribution to the Photon spectrum in a sample of interest. Traditionally Photon fake rate is defined as a probability for a jet to be misidentified as Photon and is calculated via dividing Photon $p_{T}$ spectrum by jet $p_{T}$ spectrum in a special QCDenriched sample. To apply it in the analysis for predicting Photon background from QCD one needs to multiply fake rate by the inclusive jet spectrum derived either from data (preferred) or from the Monte Carlo.

This approach is a very natural one. However it leads to several problems both in the measurement of the fake rate and in analysis applications:

1. Difference in the Photon and jet energy scale makes it hard to define the fake rate as a function of $p_{T}$.

2. Interpreting the spectrum based on the fake rate multiplication may be difficult due to energy scale uncertainty. 
3. Effects of the trigger selection in the fake rate measurement sample:

- EM triggers can not be used since they would bias the sample towards high Photon content;

- Current DØ jet triggers typically turn on only at high $p_{T}$ and may contain more subtle additional trigger biases;

- Muon triggers make an excellent choice since they provide completely unbiased Photon sample. However, the statistics of Muon triggers is limited.

More on the fake rate calculated with respect to jets can be found in [8]. Many the above problems can be avoided if we redefine Photon fake rate as a probability for a Loose EM object to pass Photon selection criteria and modify its measurement and further use accordingly:

1. The EM scale is the only energy scale present in the measurement;

2. The $p_{T}$ dependence of predicted background spectrum is well defined;

3. Basic EM triggers can be used.

With this definition the entire QCD background (from jets and from final state Photons) would be accounted for, since both sources contribute to the denominator of the fake rate.

\subsubsection{Fake Rate Measurement Sample}

We select the data sample that consists of events with exactly one Loose EM object. To ensure that this sample is dominated by the $\mathrm{QCD}$ jet and $\gamma$ production, we veto the events with more than one Loose EM object (potential $Z / \gamma^{*} \rightarrow e e$ ), as well as events with the Missing Transverse Energy above $15 \mathrm{GeV}$ (potential $W \rightarrow e \nu$ ). Finally we make sure that there is no $Z / W$-like Jacobian peak in the Photon/Electron distributions.

A trigger for selecting the fake rate measurement sample is chosen to be consistent with the trigger used for selecting the sample to which the fake rate will be applied to calculate QCD background (di-LooseEM sample): 
- the di-LooseEM sample was selected with 2EM_HI trigger, which requires two EM trigger towers above $10 \mathrm{GeV}$ at $\mathrm{L} 1$ and one loose L3 Object above $10 \mathrm{GeV}$ at L3.

- the fake rate measurement sample is selected with EM_LO trigger, which requires one EM trigger tower above $5 \mathrm{GeV}$ at $\mathrm{L} 1$ and one loose $\mathrm{L} 3$ Object above $10 \mathrm{GeV}$ at L3.

The $p_{T}$ range in which we are interested to measure the fake rate is the characteristic Photon $p_{T}$ range in Topcolor Higgs diphoton decays, shown,e.g. in Fig. 4.9 for $m_{\mathrm{h}}=120 \mathrm{GeV}$. Due to

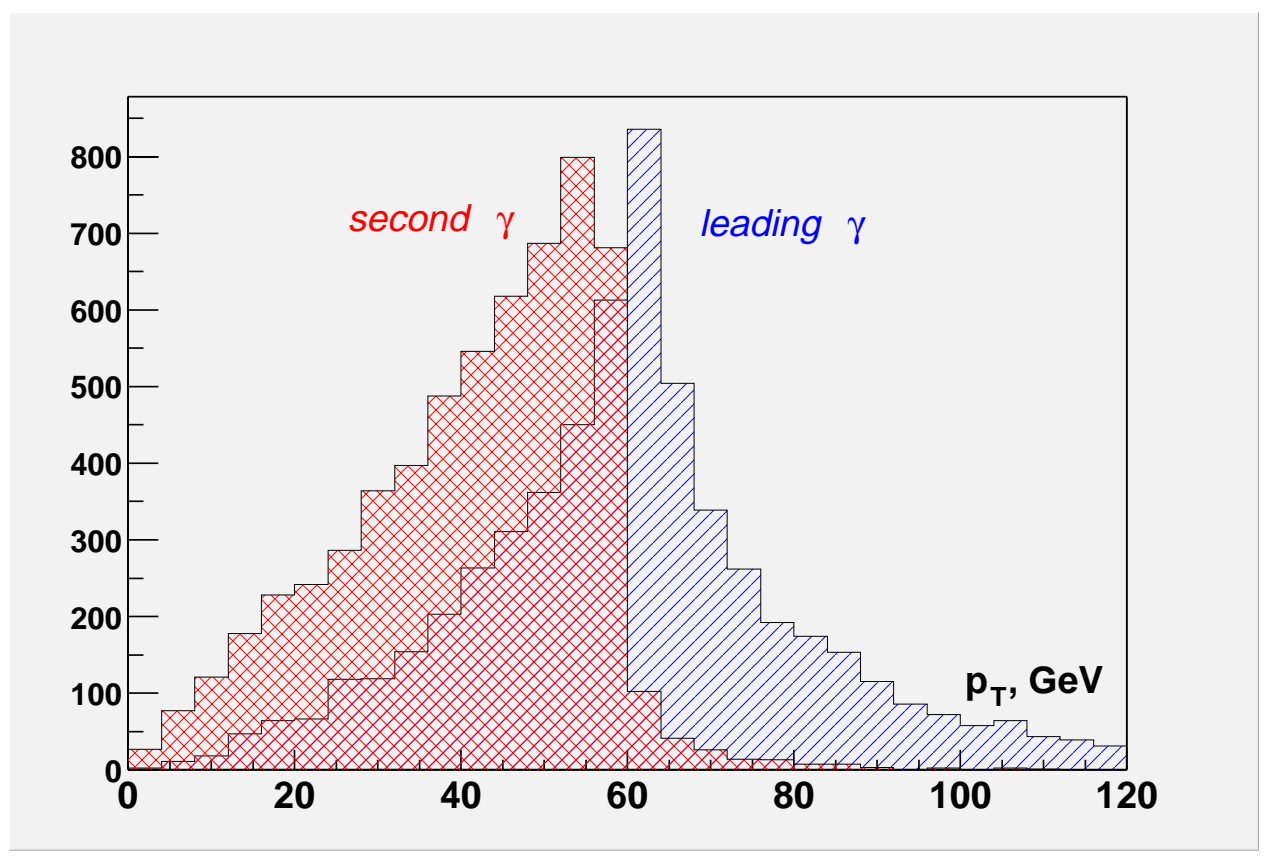

Figure 4.9: The $p_{T}$ distribution for the generator level Photons in the decay of a $120 \mathrm{GeV}$ Topcolor Higgs. Leading (in $p_{T}$ ) Photon shown with the diagonal hatching, trailing Photon shown with the cross-hatching.

limitations (EMID deficiencies at low $p_{T}$, statistics at high $p_{T}$ ) we consider the $[20,100] \mathrm{GeV}$ range. Still, since the EM_LO trigger is heavily prescaled statistics are not adequate at high $p_{T}$. To increase statistics we complement the sample with selection of an unprescaled EM_HI trigger, which requires one EM trigger tower above $10 \mathrm{GeV}$ at $\mathrm{L} 1$ and requires one loose L3 Object above $30 \mathrm{GeV}$ at L3. On the other hand, the high $p_{T}$ threshold of this trigger at the L3 prevents us from using it at the 
low end of the $p_{T}=[20,100] \mathrm{GeV}$ range. Eventually, we use EM_LO for $p_{T}=[20,40] \mathrm{GeV}$ and EM_HI for $p_{T}=[40,100] \mathrm{GeV}$. We verify reasonable agreement in the overlap region around $40 \mathrm{GeV}$ and use combined EM_LO+EM_HI sample for the final fake rate calculation.

\subsubsection{Results}

Figs. 4.11 and 4.10 show the results for the End and Central Calorimeter, respectively. Plots include $p_{T}$ distributions for the Loose EM objects, Photons, and Electrons, as well as fake rates for each trigger subsample and for the combined EM_LO+EM_HI sample.
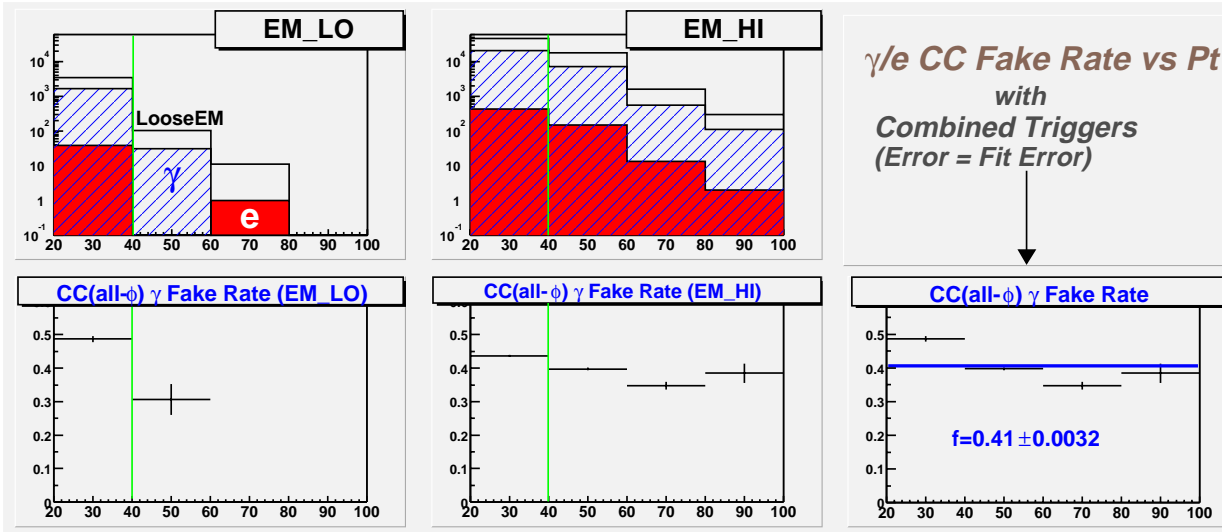

(Error = Fit Error)
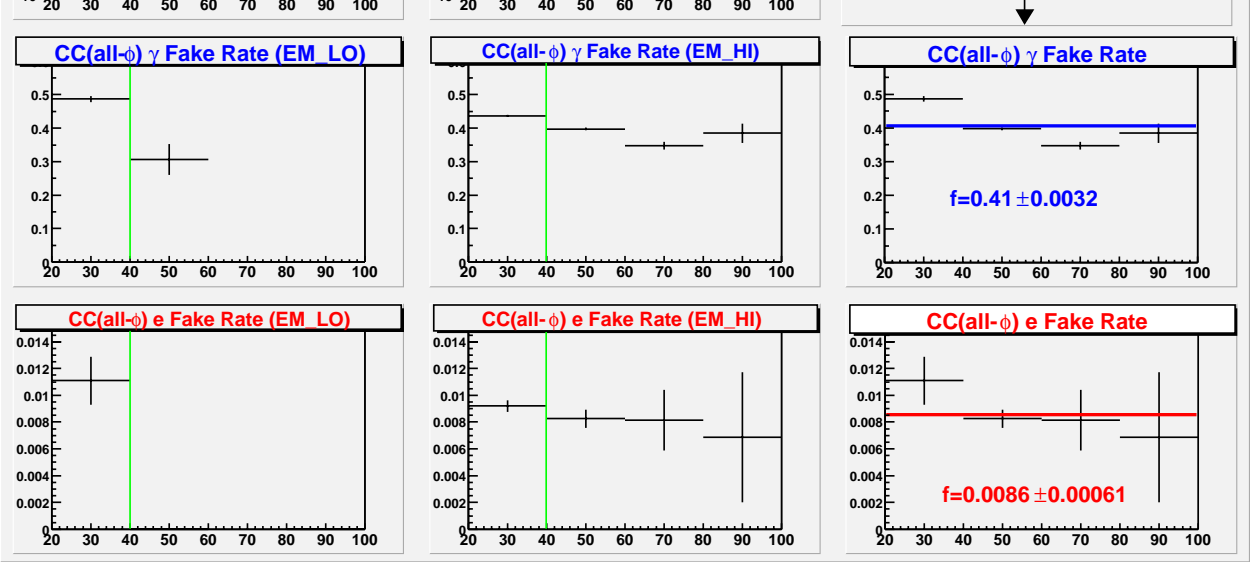

Figure 4.10: The $p_{T}$ distributions and fake rates vs $p_{T}$ in the Central Calorimeter (CC). Top row left - $p_{T}$ distributions of Loose EM objects (open histogram), Photons (light hatched), and Electrons (dark hatched) in the EM_LO subsample; Top row middle $-p_{T}$ distributions for the Loose EM objects (open histogram), Photons (light hatched) and Electrons (dark hatched) in the EM_HI subsample; Middle row left - Photon fake rate for the EM_LO subsample; Bottom row left - Electron fake rate for the EM_LO subsample; Middle row middle - Photon fake rate for the EM_HI subsample; Bottom row middle - Electron fake rate for the EM_HI subsample; Middle row right - Photon fake rate for the combined sample; Bottom row right - Electron fake rate for the combined sample 

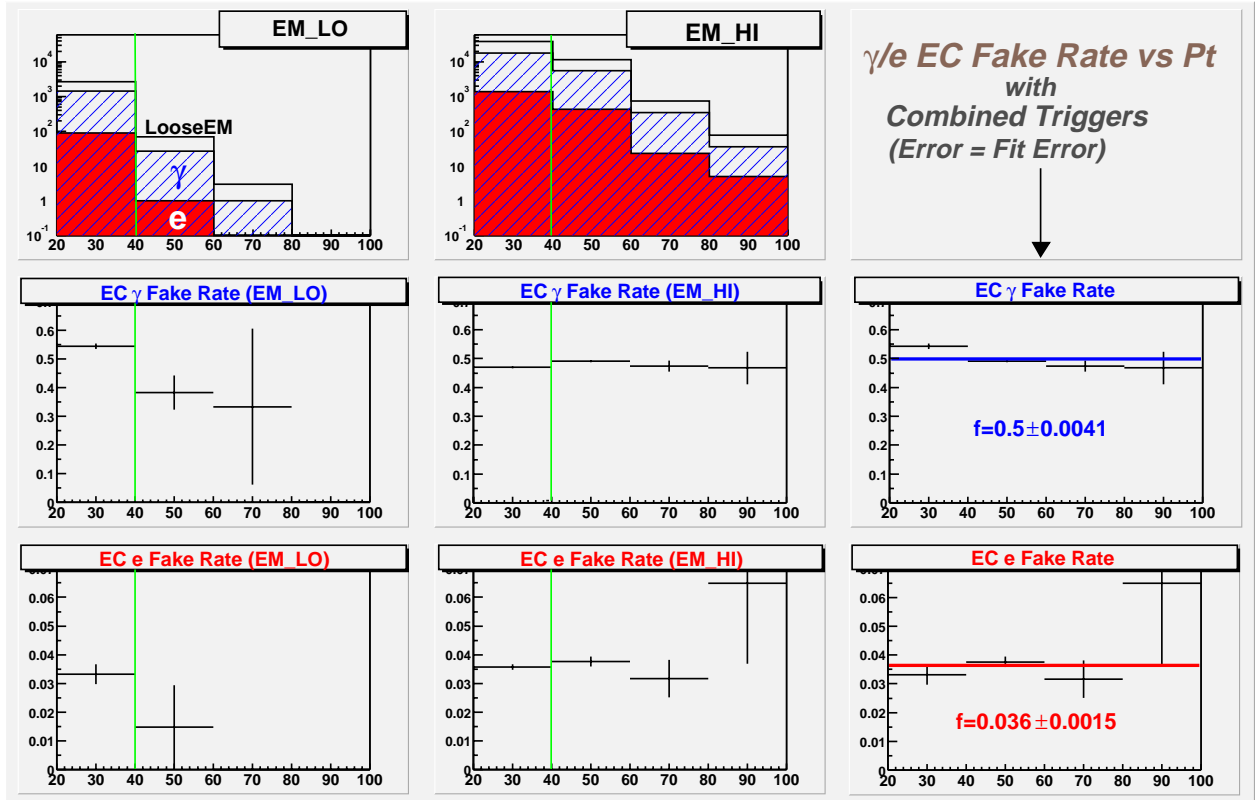

Figure 4.11: The $p_{T}$ distributions and fake rates vs $p_{T}$ in the End Cap Calorimeter (EC). Top row left $-p_{T}$ distributions of Loose EM objects (open histogram), Photons (light hatched), and Electrons (dark hatched) in the EM_LO subsample; Top row middle $-p_{T}$ distributions for the Loose EM objects (open histogram), Photons (light hatched) and Electrons (dark hatched) in the EM_HI subsample; Middle row left - Photon fake rate for the EM_LO subsample; Bottom row left - Electron fake rate for the EM_LO subsample; Middle row middle - Photon fake rate for the EM_HI subsample; Bottom row middle - Electron fake rate for the EM_HI subsample; Middle row right - Photon fake rate for the combined sample; Bottom row right - Electron fake rate for the combined sample 
In case of the Photon fake rate the uncertainty is dominated by the systematics due to the $p_{T}$ dependence of the fake rate, the origin of which is not quite understood. We estimate the systematic error as fit error $\times \sqrt{\frac{\chi^{2}}{N \text { degrees of freedom }}}$. Relative uncertainties are found to be:

CC Photon fake rate uncertainty: $4.9 \%$

EC Photon fake rate uncertainty: $2.4 \%$

CC Electron fake rate uncertainty: $7.0 \%$

EC Electron fake rate uncertainty: $4.2 \%$

\subsection{EMID Efficiency}

The EMID Efficiency $\epsilon(E M)$ is defined as a probability for a Loose EM object to pass the EM object selection criteria. It can be estimated either from Monte Carlo simulated samples or from real data. We estimate it from the data using the $Z$-mass peak:

- To obtain a $Z$-peak with a relatively small background we require a track match for one of the Loose EM objects (tag object), leaving the other object (probe object) unbiased.

- Fit the $Z$-peak to obtain the number of signal events (denominator in the EMID efficiency formula).

- Apply the EM object ID cut to the probe object, fit the $Z$-peak to obtain the number of signal events (numerator in the EMID efficiency formula).

- CC EMID efficiency was calculated using CCCC di-LooseEM events, while EC EMID efficiency was calculated using CCEC events.

- To estimate systematic error due to the uncertainty in describing the background, we repeat the measurement applying a tight calorimeter based cut $($ Isolation $<0.015)$ to the tag object. EMID efficiency is calculated as the average of the two measurements, while half of the difference between them is used as a systematic error.

CCCC and CCEC diphoton mass distributions are shown in Figs. 4.12 and 4.13, respectively. 

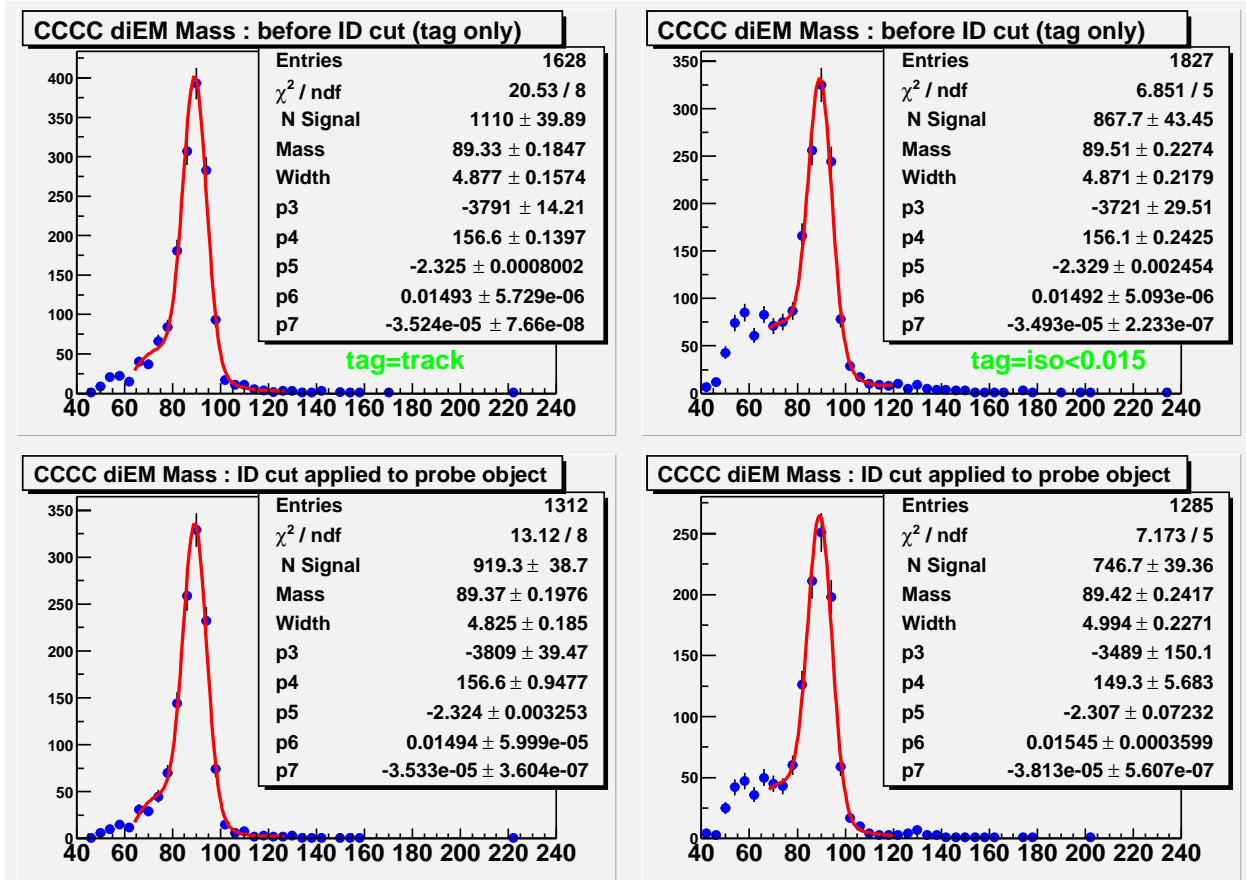

Figure 4.12: CCCC diphoton mass distributions (in GeV) for the CC EMID Efficiency measurement. Left column corresponds to the case when the tag object is required to have a track match, whereas in the right column uses the calorimeter based cut (Isolation < 0.015). Top plots shows the denominator mass distributions i.e. when a cut is applied to the tag object only. Bottom plots show numerator mass distributions i.e. when (EM) ID cut is applied to the probe object. 

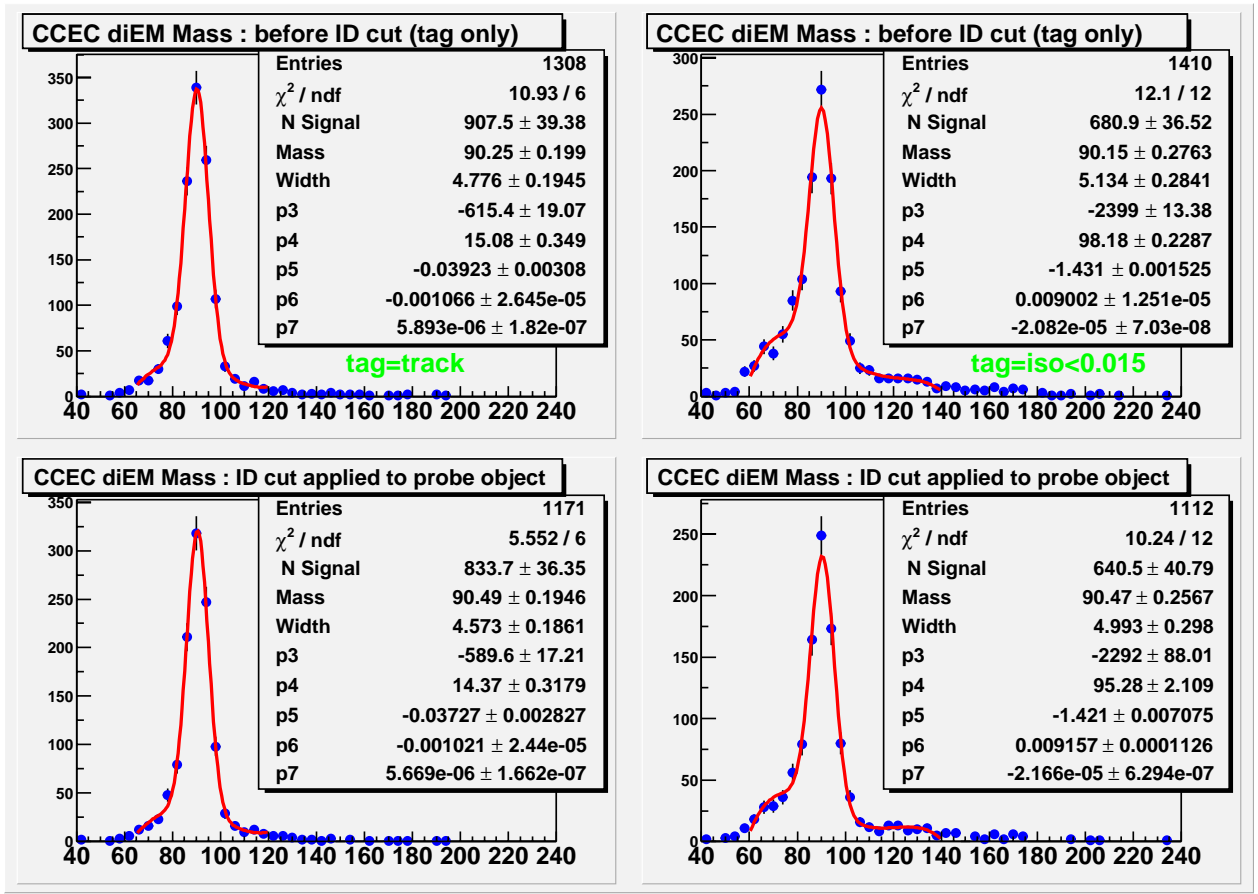

Figure 4.13: CCEC diphoton mass distributions (in GeV) for the EC EMID Efficiency measurement. Left column corresponds to the case when the tag object is required to have a track match, whereas in the right column uses the calorimeter based cut (Isolation < 0.015). Top plots shows the denominator mass distributions i.e. when a cut is applied to the tag object only. Bottom plots show numerator mass distributions i.e. when (EM) ID cut is applied to the probe object. 
Efficiency is found to be: $84.4 \pm 1.1($ stat $) \pm 1.6($ syst $) \%(\mathrm{CC}) ; 93.0 \pm 0.9($ stat $) \pm 1.1($ syst $) \%(\mathrm{EC})$

\subsection{Tracking Efficiency}

Let us define Tracking Efficiency $\epsilon$ (track) as the efficiency of finding a track and matching it to an electron. "Default" EMID software match with axial tracks was used. We calculate Tracking Efficiency based on $Z \rightarrow e e$ events using the following formula:

$$
\epsilon(\text { track })=\frac{2 N_{2}+N_{1}}{2 N_{\mathrm{all}}}
$$

where $N_{\text {all }}$ is the total number of di-EM events in the $Z$-peak, $N_{2}, N_{1}$ are the numbers of events with exactly two matches and exactly one match, respectively. Figures 4.14, 4.15, and 4.16 show corresponding di-EM mass distributions for CCCC, CCEC, ECEC events from which $N_{2}, N_{1}, N_{\text {all }}$ are obtained by subtracting continuum background under the $Z$ resonance. The values of $N_{2}, N_{1}$, $N_{\text {all }}$ are shown in Table 4.2. Alternatively, $\epsilon$ (track) can be calculated as $\sqrt{N_{2} / N_{\text {all }}}$, which we use as a

\begin{tabular}{cccc}
\hline Number of events & CCCC & CCEC & ECEC \\
\hline$N_{\text {all }}$ & $1225 \pm 45.71$ & $1168 \pm 46.93$ & $246.8 \pm 18.76$ \\
$N_{1}$ & $556 \pm 28.95$ & $510.5 \pm 27.0$ & $94.18 \pm 10.87$ \\
$N_{2}$ & $613 \pm 30.46$ & $476.4 \pm 26.3$ & $110.1 \pm 11.28$ \\
\hline
\end{tabular}

Table 4.2: $N_{\text {all }}, N_{2}, N_{1}$

cross-check. A major source of systematic error in this measurement is the uncertainty in describing the background shape under the Z-peak. One way to estimate this error is to use the difference in the efficiency obtained via $\epsilon($ track $)=\left(2 N_{2}+N_{1}\right) / 2 N_{\text {all }}$ and $\sqrt{N_{2} / N_{\text {all }}}$. This difference would reflect the background uncertainty coming in via $N_{1}$ as well as other possible systematic effects, e.g. correlation of tracking efficiency in 2-track events. To verify the validity of this approach, we also calculated error that comes specifically from the background shape uncertainty by changing the background fit functions. In Figs. 4.14, 4.15, and 4.16 a straight line was used to describe the 


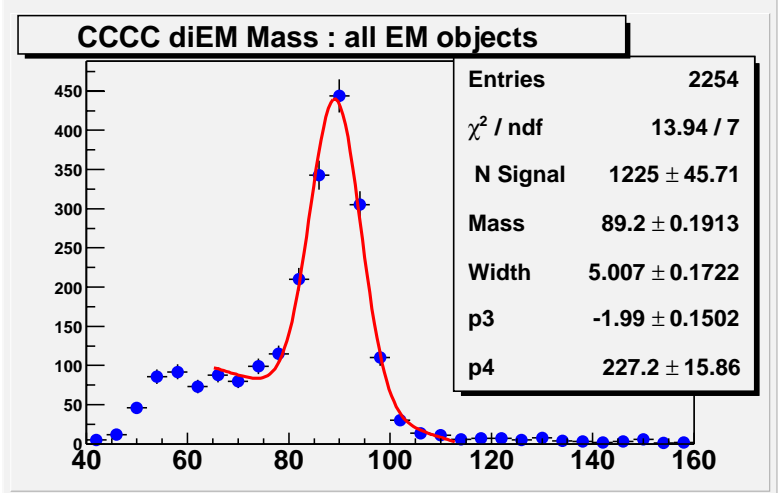

\section{CCCC diEM Mass : exactly one track match}
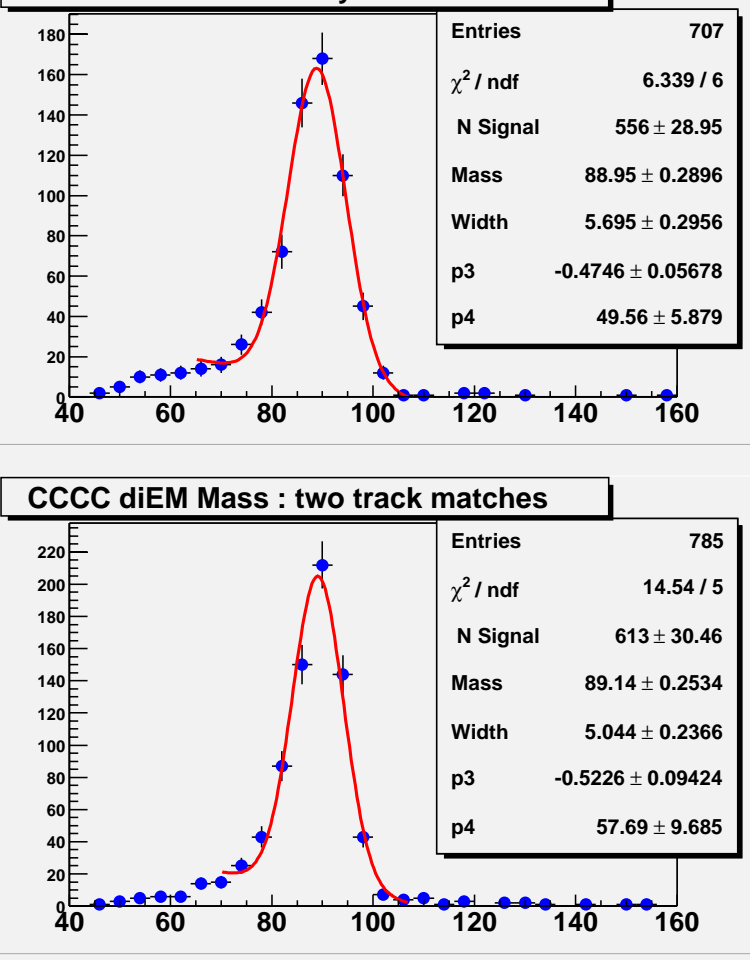

Figure 4.14: di-EM mass distributions for Tracking Efficiency measurement in CCCC events. The top plot shows the mass spectrum and the fit for the whole sample; the middle plot corresponds to the events with exactly one track match; the bottom plot shows the events in which both EM objects are matched to tracks. Linear function was used to fit the background. 


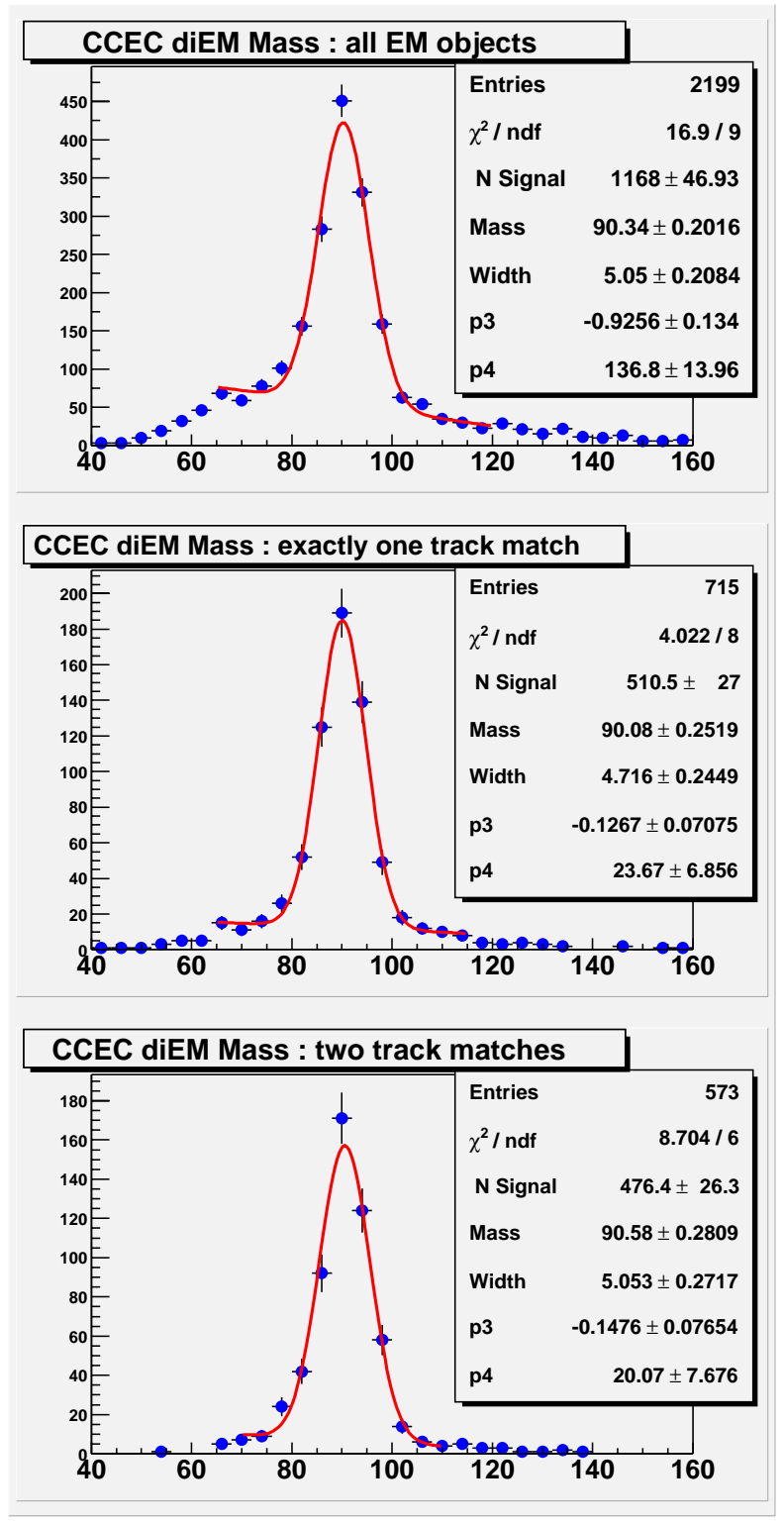

Figure 4.15: di-EM mass distributions for Tracking Efficiency measurement in CCEC events. The top plot shows the mass spectrum and the fit for the whole sample; the middle plot corresponds to the events with exactly one track match; the bottom plot shows the events in which both EM objects are matched to tracks. Linear function was used to fit the background. 


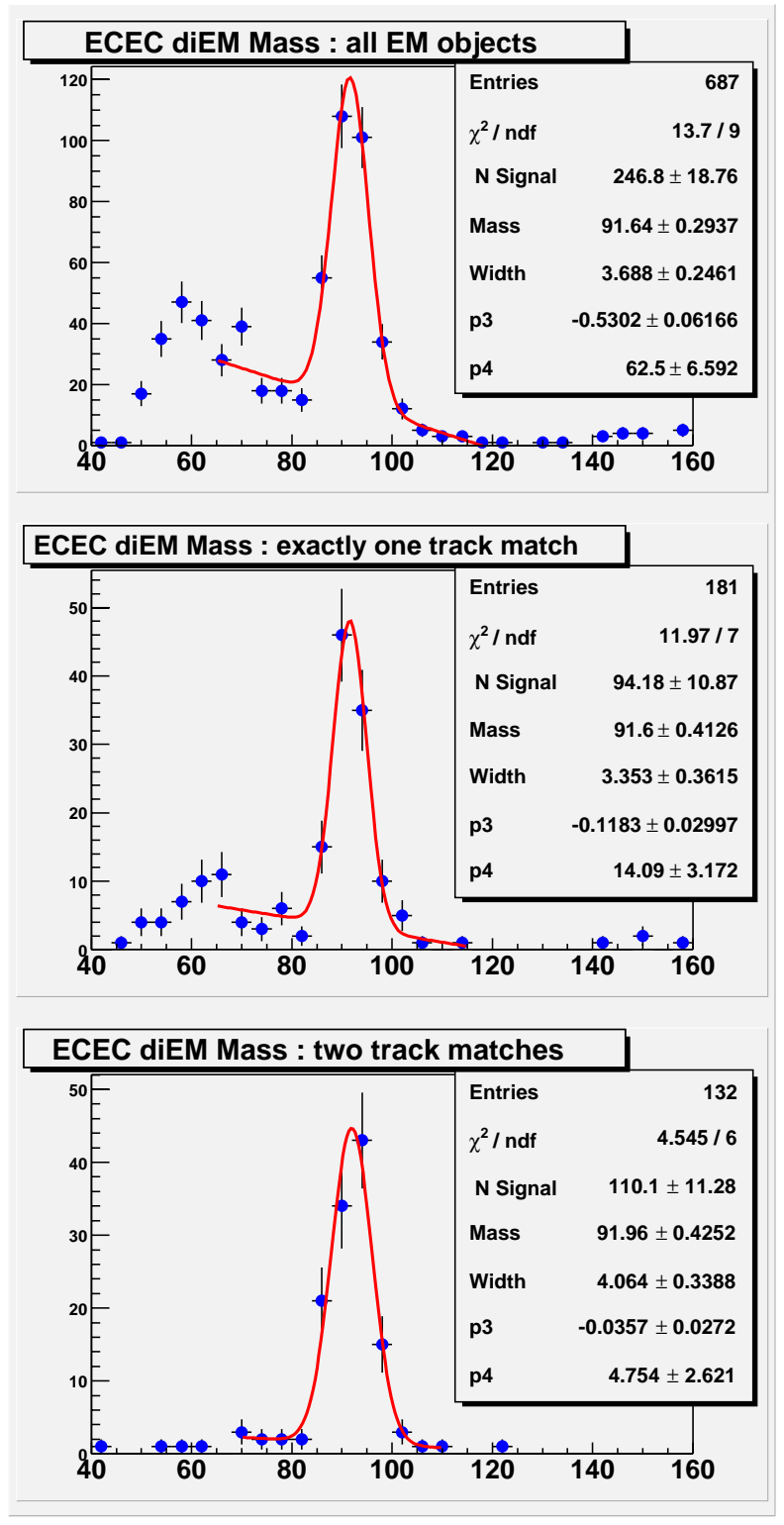

Figure 4.16: di-EM mass distributions for Tracking Efficiency measurement in ECEC events. The top plot shows the mass spectrum and the fit for the whole sample; the middle plot corresponds to the events with exactly one track match; the bottom plot shows the events in which both EM objects are matched to tracks. Linear function was used to fit the background. 
background. As a cross-check, we used an exponential function in the case of CCEC and ECEC, and the polynomial in the case of CCCC. The results of this measurement and comparison with the standard method are given in Appendix A.

The tracking efficiencies are found to be:

$\epsilon($ track $)=72.7 \pm 0.9($ stat $) \pm 2.0($ syst $) \%$ CCCC;

$\epsilon($ track $)=62.6 \pm 1.0($ stat $) \pm 1.2($ syst $) \%$ CCEC;

$\epsilon($ track $)=63.7 \pm 2.2($ stat $) \pm 3.1($ syst $) \%$ ECEC; 


\section{Chapter 5}

\section{Analysis}

It's cold there... and there is snow,

...the tripod of an icy Pythia

is wrapped in the haze of a white snowstorm...

The stretched scars of impetuous peaks...

No settlements. A cheerful carrousel...

it tosses the cables into a tremble at the seams.

I soar over the white pain...

And finally - synthesis. Finally - a result.

Lina Kostenko, "The Summit of Sorrow"

How far the story matters to anyone but myself

depends on the degree to which others have

experienced what I call "joy"

C.S. Lewis, "Surprised by Joy"

This chapter describes the search for Fermiophobic Higgs and Topcolor Higgs (see the end of Section 2.3.2), assuming SM-like couplings and considering the branching fraction into photons to 
be a free parameter [86]. The search is done in the inclusive $h \rightarrow \gamma \gamma+X$ channel using diphoton invariant mass as the primary analysis variable.

\subsection{Data and Event Selection}

\subsubsection{Dataset}

We used the data collected by the DØ detector between October 2002 and February 2003. We used the sample containing two EM objects ${ }^{1}$ selected by the DØ New Phenomena Physics group [87]. Trigger selection was done with an unprescaled 2EM_HI trigger, which requires two EM trigger towers with $E_{T}$ above $10 \mathrm{GeV}$ at $\mathrm{L} 1$ and requires one loose L3 EM Object with $E_{T}$ above $10 \mathrm{GeV}$ at L3. The loose L3 EM Object is required to pass only a loose EM fraction cut. Trigger $|\eta|$ coverage extends up to 2.4. The integrated luminosity of this sample is $51.8 \pm 5.2 \mathrm{pb}^{-1}$, as reported by the DØ luminosity group.

\subsubsection{Di-LooseEM Data Sample}

Basic offline selection requires two Loose EM objects with $E_{T}$ above $25 \mathrm{GeV}$. In what follows, the set of events passing this selection will be referred to as di-LooseEM sample.

Since the Loose EM object definition contains the EM shower shape cut and no other types of objects are used in the analysis (e.g. jets or Missing $E_{T}$ ), data quality is ensured automatically by virtue of the selection and therefore only minimal "bad" run veto is applied to avoid unnecessary reduction in statistics. Nevertheless we make a cross-check with a subsample in which bad Jet/Missing $E_{T}$ runs are excluded. This luxury will no longer be available when jet requirements are included in the analysis.

\footnotetext{
${ }^{1}$ see Section 4.5 for definitions of the analysis objects in this chapter
} 


\subsubsection{Final Data Sample}

The final data sample is obtained from the di-LooseEM sample by applying to it di-Photon event selection.

\subsection{Backgrounds}

\subsubsection{Sources of Background}

Major sources of background to $h \rightarrow \gamma \gamma$ are:

- Drell-Yan $Z / \gamma^{*} \rightarrow e e$ events in which both Electrons are misidentified as Photons (the diagram for this process is shown in Figure 5.1);

- QCD events that in the final state have:

(i) two Photons (Figure 5.2); or

(ii) a Photon and a jet misidentified as Photon (examples in Figure 5.3); or

(iii) two jets misidentified as Photons (examples in Figure 5.4).

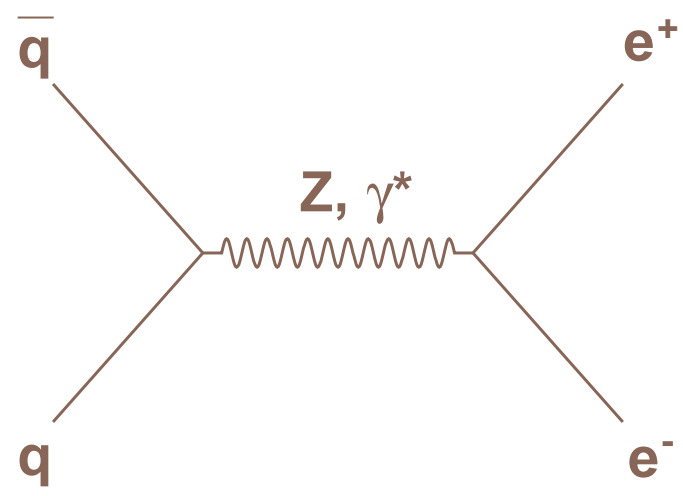

Figure 5.1: Drell-Yan process diagram.

\subsubsection{Predicting the Drell-Yan Background}

Drell-Yan background is calculated from di-LooseEM sample by relating di-Photons to di-Electrons via Tracking Efficiency. Derivation of the final expression is as follows: the number of di-Electron 


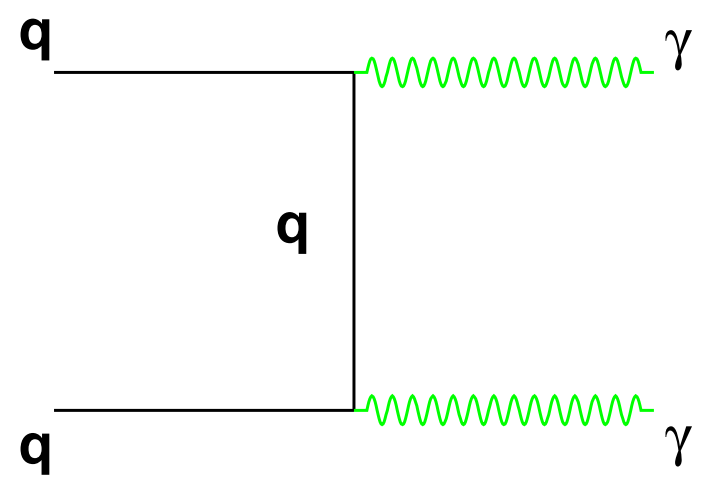

Figure 5.2: Direct diphoton process diagram.

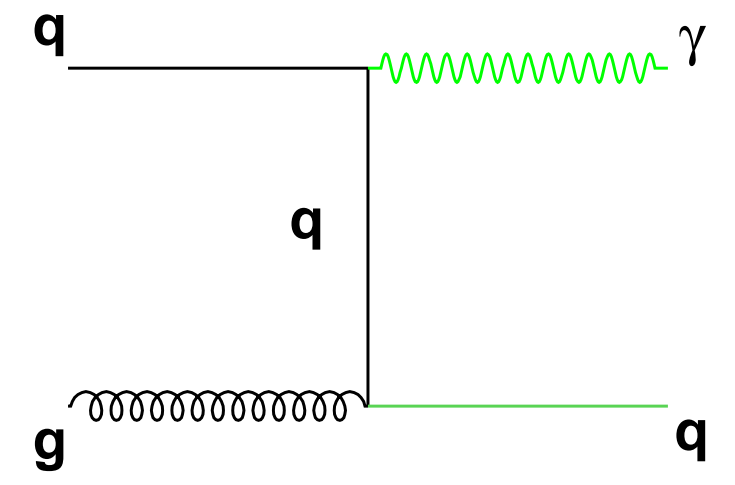

Figure 5.3: One of the $\gamma+$ jet production diagrams. 


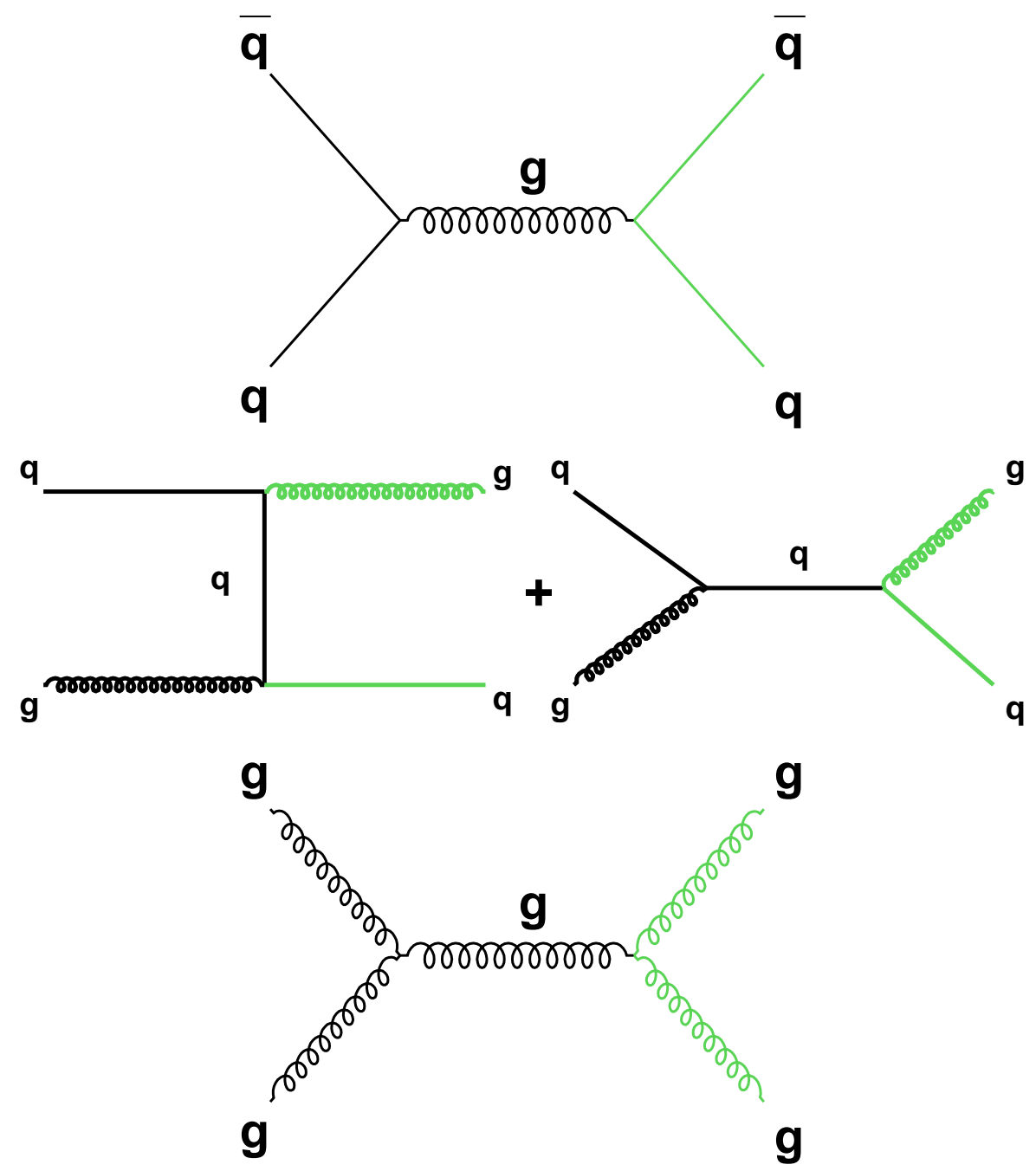

Figure 5.4: Examples of dijet production diagrams. 
and $d i$-Photon events in the sample coming from $Z / \gamma^{*} \rightarrow e e$ process is given by equations (5.1) and (5.2) respectively:

$$
\begin{gathered}
N_{\mathrm{DY}}(e e)=\sigma\left(p \bar{p} \rightarrow Z / \gamma^{*}+X\right) \times B(e e) \times L_{\mathrm{int}} \times A \times \epsilon(\text { trigger }) \times \epsilon^{2}(E M) \times \epsilon^{2}(\text { track }) \\
N_{\mathrm{DY}}(\gamma \gamma)=\sigma\left(p \bar{p} \rightarrow Z / \gamma^{*}+X\right) \times B(e e) \times L_{\mathrm{int}} \times A \times \epsilon(\text { trigger }) \times \epsilon^{2}(E M) \times(1-\epsilon(\text { track }))^{2},
\end{gathered}
$$

where $\sigma\left(p \bar{p} \rightarrow Z / \gamma^{*}+X\right)$ is the production cross-section, $\operatorname{Br}(e e)$ is the di-Electron branching fraction, $L_{\text {int }}$ is the integrated luminosity, $A$ is the combined geometric and kinematic acceptance, $\epsilon($ trigger $)$ is the trigger efficiency, $\epsilon(E M)$ is the EMID Efficiency introduced in Section 4.7, and $\epsilon($ track $)$ is the Tracking Efficiency introduced in Section 4.8 .

From (5.1) and (5.2) follows (5.3):

$$
N_{\mathrm{DY}}(\gamma \gamma)=N_{\mathrm{DY}}(e e) \times \frac{(1-\epsilon(\text { track }))^{2}}{\epsilon^{2}(\text { track })}
$$

The right-hand side of this equation still has to be reduced to measurable quantities. $N_{\mathrm{DY}}(e e)$ can be written as the difference between the total number of $d i$-Electrons observed, $N(e e)$, and the number of di-Electrons coming from sources other than Drell-Yan $N_{\text {nonDY }}(e e)$ :

$$
N_{\mathrm{DY}}(e e)=N(e e)-N_{\mathrm{nonDY}}(e e)
$$

The dominant non Drell-Yan source of Electrons is misidentification of QCD jets. Therefore non Drell-Yan Electrons are Fake Electrons defined in Section 4.6 and their number can be estimated by applying the Electron fake rate calculated with respect to Loose EM objects to the di-LooseEM sample:

$$
N(n o n D Y e e)=N(d i-L o o s e E M) \times f^{2}(e)
$$

where $f(e)$ is the Electron fake rate. Combining (5.4) and (5.5) gives:

$$
N_{\mathrm{DY}}(e e)=N(e e)-N(d i-L o o s e E M) \times f^{2}(e)
$$


Combining (5.6) with (5.3) we obtain the final expression for the Drell-Yan Background in the $\gamma \gamma$ channel:

$$
N_{\mathrm{DY}}(\gamma \gamma)=\left\{N(e e)-N(\text { di }- \text { LooseE } M) \times f^{2}(e)\right\} \times \frac{(1-\epsilon(\text { track }))^{2}}{\epsilon^{2}(\text { track })} .
$$

\subsubsection{Predicting QCD $\gamma \gamma$ Background}

QCD $\gamma \gamma$ Background is calculated by applying the Photon fake rate calculated with respect to Loose

EM objects to the di-LooseEM sample, after subtraction of the Drell-Yan background:

$$
N_{\mathrm{QCD}}(\gamma \gamma)=\left\{N\left(d i-\text { LooseEM) }-N_{\mathrm{DY}}(\text { di }- \text { LooseEM })\right\} \times f^{2}(\gamma)\right.
$$

where $f(\gamma)$ is the Photon fake rate introduced in Section 4.6.

The right-hand side of this formula still needs to be reduced to measurable quantities. $N_{\mathrm{DY}}(d i-$ LooseEM) can be related to Drell-Yan di-Electrons $N_{\mathrm{DY}}(e e)$ via tracking efficiency $\epsilon($ track) (Section 4.8) and EMID efficiency $\epsilon(E M)$ (Section 4.7):

$$
N_{\mathrm{DY}}\left(d i-\text { LooseEM) }=\frac{N_{\mathrm{DY}}(e e)}{\epsilon^{2}(E M) \times \epsilon^{2}(\text { track })} .\right.
$$

Combining (5.8) with (5.9) and using (5.6) from the previous section for $N_{\mathrm{DY}}(e e)$, we obtain the final expression for the QCD $\gamma \gamma$ background:

$$
N_{\mathrm{QCD}}(\gamma \gamma)=\left\{N(d i-\text { LooseEM })-\frac{\left[N(e e)-N(d i-\text { LooseEM }) \times f^{2}(e)\right]}{\epsilon^{2}(E M) \times \epsilon^{2}(\text { track })}\right\} \times f^{2}(\gamma) .
$$

\subsubsection{Summary of Background Estimates}

Note that both backgrounds are estimated from the di-LooseEM data sample (the very same sample that is used for the final event selection) using the measured values of Tracking Efficiency, EM Efficiency, Photon fake rate, and Electron fake rate. The efficiencies are calculated from the diLooseEM data sample, whereas the fake rates - from an independent (One)LooseEM data sample. More information on these measurements is provided in the corresponding sections of the Photon 
Identification Chapter.

Note that although the efficiencies and fake rates have to be calculated for a specific set of analysis object definitions, the applicability of the described procedure is not sensitive to possible variations in these definitions due to the analysis optimization. Such universality comes at a cost of possible redundancy in the final expressions. For example with the current set of cuts Electron fake rate term is negligible, and so is the Drell-Yan contribution to the QCD sample beyond the $Z$-mass region.

It is worth mentioning that the above procedure for the QCD $\gamma \gamma$ background derivation must be followed with caution. As was noted, a Loose EM object in the QCD event may come from two sources: a misidentified hadronic jet or a final state photon. These two are expected to have different distributions in the EMID variables. If there is a significant difference in relative content of Loose EM objects from jets and Loose EM objects from photons between the di-LooseEM sample and the fake rate measurement sample (EM Sample), our method is not self-consistent. The relative content is determined by the corresponding cross-sections and the probability for a hadronic jet to be reconstructed as a Loose EM object. Acceptance cuts do not affect it since no difference is expected in the kinematics of a purely hadronic QCD process compared to a QCD process with photon(s) in the final state.

In our case it turns out that this is not a problem since for the current jet $\rightarrow$ LooseEM probability for both samples is dominated by Loose EM objects coming from hadronic jets. However, this may be something to keep in mind for this analysis in the future.

\subsection{Mass Distributions and Event Counts}

The $\gamma \gamma$ invariant mass distributions for the data and predicted background, as well as the event counts are shown in Fig. 5.5 for the three event topologies. The logarithmic vertical scale is used in Fig. 5.5. Figure 5.6 shows the same plots using linear vertical scale. The CCCC and CCEC distributions exibit a good agreement between the data and the predicted SM background, some discrepancies in the ECEC events are still to be understood. All in all, the measured $\gamma \gamma$ spectrum is 
in agreement with the expected SM background and can be used for deriving Higgs cross-section limits. Figure 5.7 shows the same distributions for the subsample of data from which bad JET/Missing $E_{T}$ runs are excluded. No significant difference is observed and therefore the main analysis is based on the full sample.

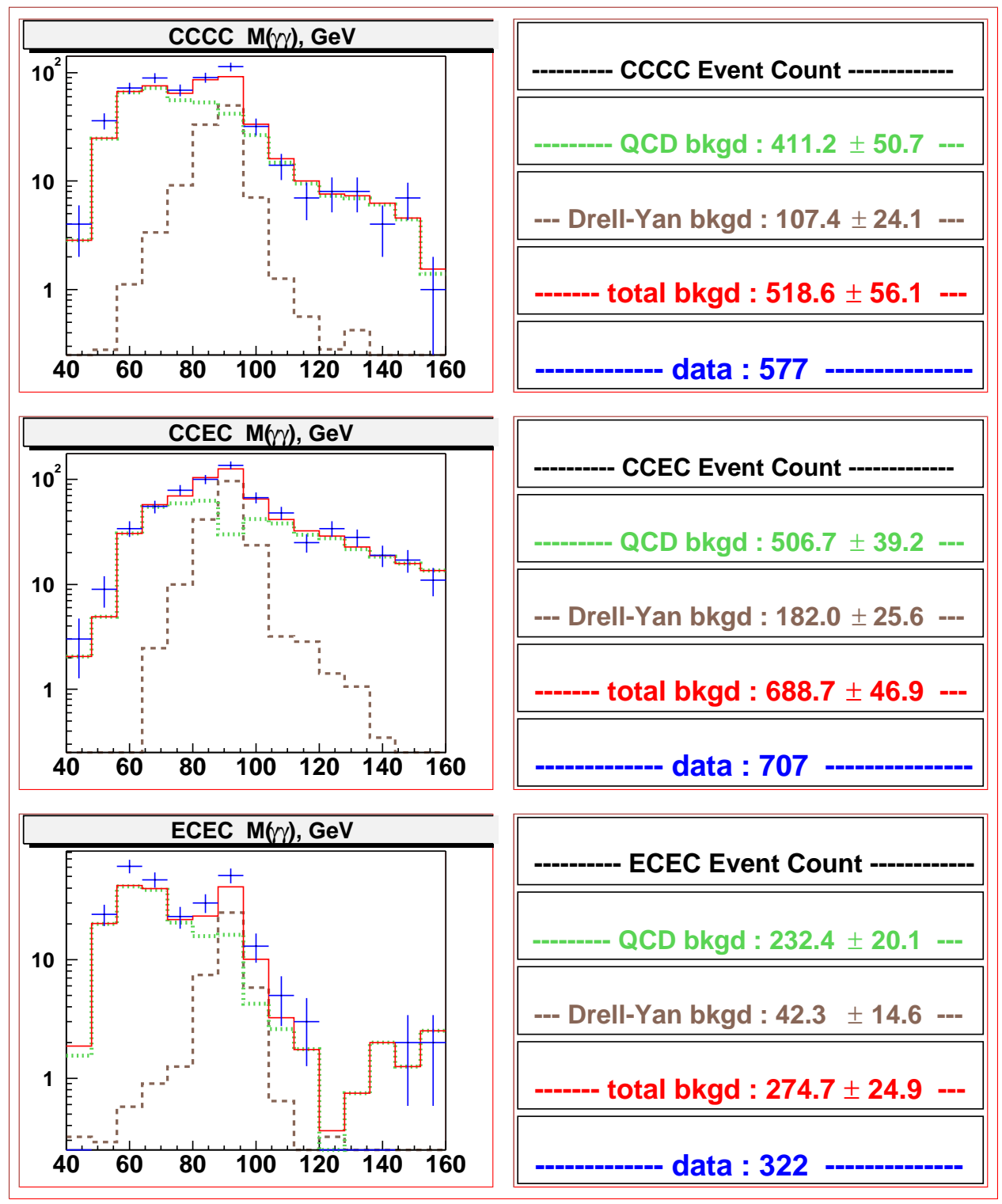

Figure 5.5: Distributions in the $\gamma \gamma$ invariant mass and event counts for different event topologies. Logarithmic vertical scale is used. Points $-\gamma \gamma$ spectrum observed in data, solid line - total SM background prediction, dotted line - QCD background, dashed line - Drell-Yan background 

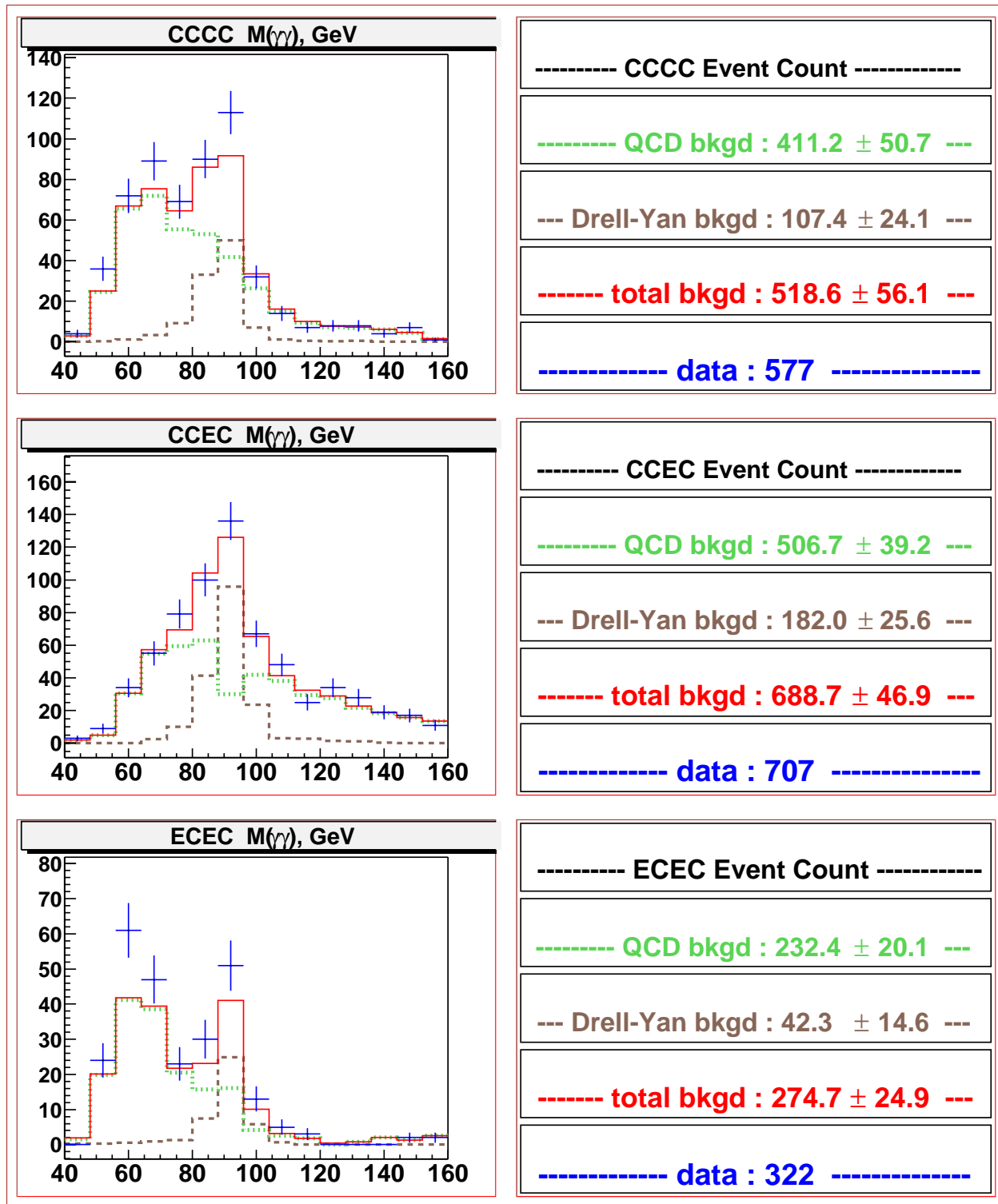

Figure 5.6: Same distributions as in previous Figure ( $\gamma \gamma$ invariant mass) are shown with liner vertical scale. Points $-\gamma \gamma$ spectrum observed in data, solid line - total SM background prediction, dotted line - QCD background, dashed line - Drell-Yan background 

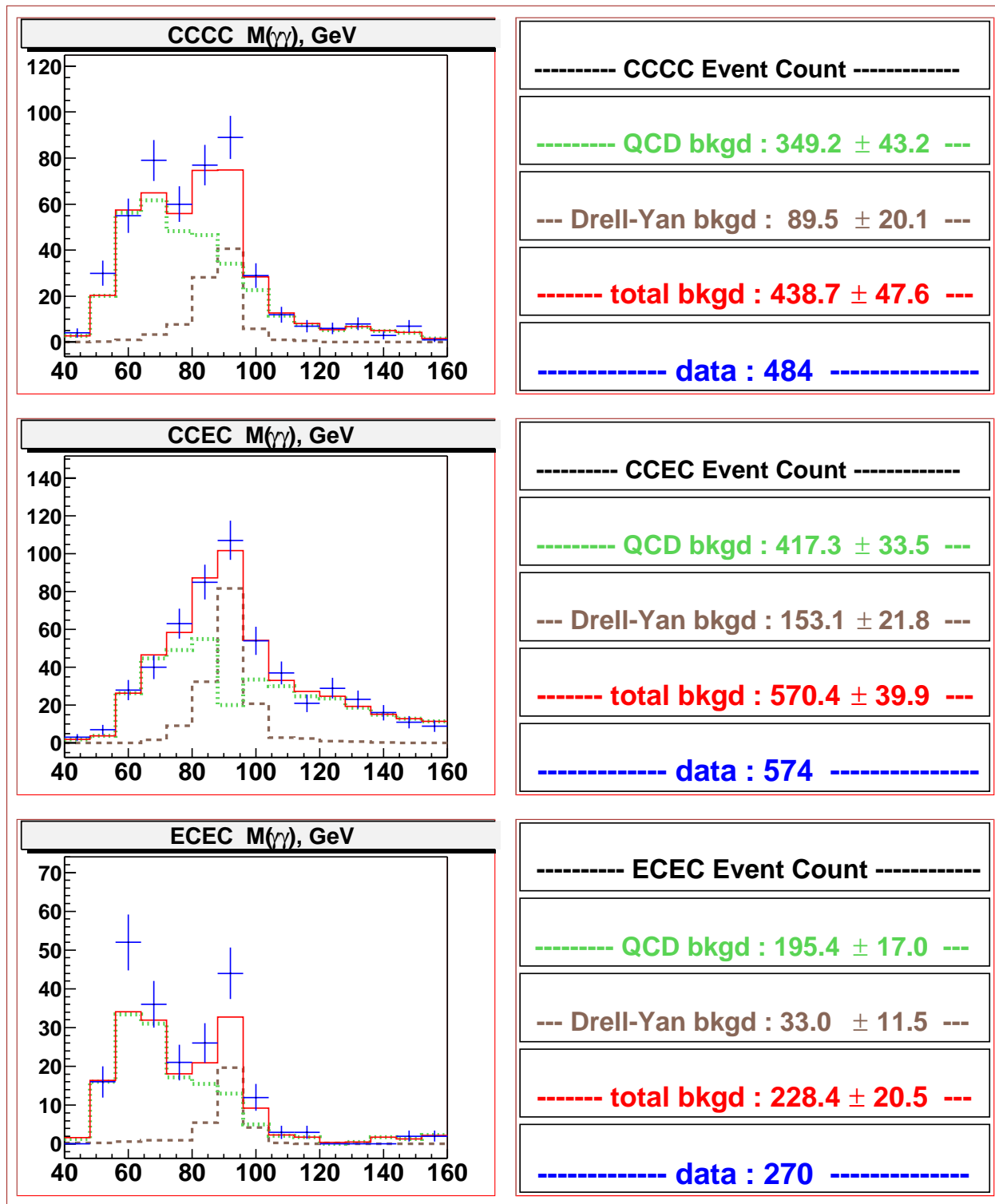

Figure 5.7: Same distributions as in previous Figure ( $\gamma \gamma$ invariant mass) are shown for the subsample of data in which bad JET/Missing $E_{T}$ runs are removed. No significant difference with the full sample is observed. Points $-\gamma \gamma$ spectrum observed in data, solid line - total SM background prediction, dotted line - QCD background, dashed line - Drell-Yan background 


\subsection{Monte Carlo Signal Samples}

When calculating cross-section limits, we rely on the $h \rightarrow \gamma \gamma$ Monte Carlo for the following:

- reconstructed $h \rightarrow \gamma \gamma$ mass width;

- acceptance;

- signal event topology;

- track veto efficiency.

We use MC samples generated for 60, 70, 80, 90, 100, $120 \mathrm{GeV}$ Higgs mass points. The samples were produced with the PYTHIA event generator [88] and standard DØ detector simulation and reconstruction software. We generated samples for individual production processes (gluon fusion, W/Z associated production, W/Z fusion) out of which Fermiophobic Higgs and Topcolor Higgs samples were composed using the cross-section ratios between the individual production processes.

\subsection{Event Topology and EM Efficiency}

$h \rightarrow \gamma \gamma$ events tend to be central. For example, in the case of $M_{\mathrm{h}}=60 \mathrm{GeV}$, the CCCC events (both Photons in Central Calorimeter) constitute $71 \%$ of total events, while CCEC and ECEC contribute $18 \%$ and $11 \%$, respectively. Since the ECEC fraction of signal events is small and the ECEC $\gamma \gamma$ mass spectrum is least understood, we exclude the ECEC events from further analysis and consider only:

1. CCCC only events;

2. CCEC only events.

Our strategy is first to derive the cross-section limits for the CCCC sample as it has much higher acceptance and then try to improve the limits by combining likelihoods for the CCCC and CCEC samples. The $h \rightarrow \gamma \gamma$ efficiency (per event) is estimated as $\epsilon^{2}(C C)$ and $\epsilon(C C)$ epsilon $(E C)$ for the CCCC and CCEC subsamples respectively. $\epsilon(C C)$ and $\epsilon(E C)$ are the EM efficiencies measured in the $Z \rightarrow e e$ data with respect to the same cuts $(E M$ fraction $>0.9$, isolation $<0.2)$ as used for the 
MC Photons selection. Measurement is done with the method described in Section 4.7. Figures 5.8 and 5.9 show the corresponding mass distributions for CCCC and CCEC, respectively.
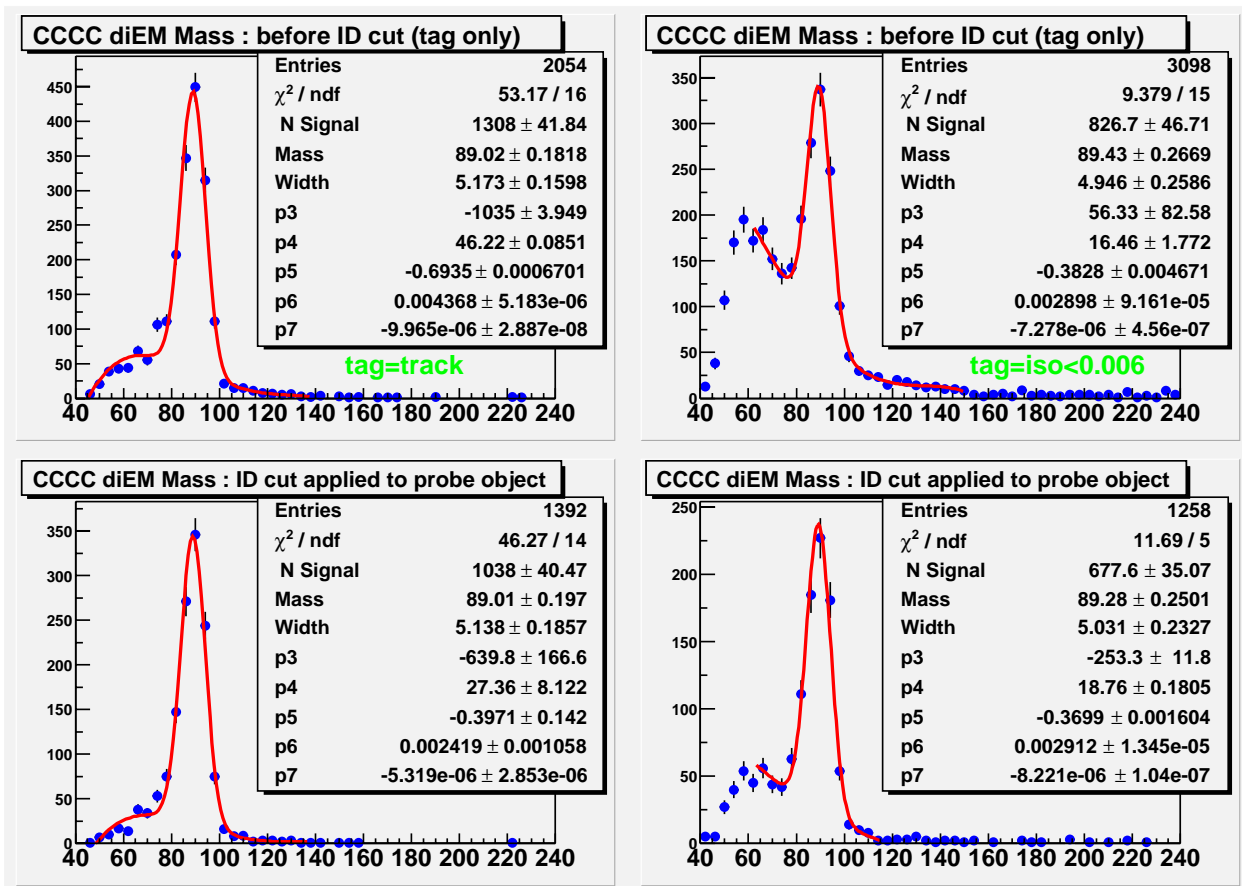

Figure 5.8: CCCC Mass distributions for the measurement of $\epsilon(C C)$. Left column corresponds to the case when the tag object is required to have a track match, whereas in the right column calorimeter based cut $($ Isolation < 0.006 ) is applied to the tag object. Top plots show the denominator mass distributions, i.e. when the cut is applied on the tag object only. Bottom plots show the numerator mass distributions, i.e. when the (EM) ID cut is applied to the probe object. 

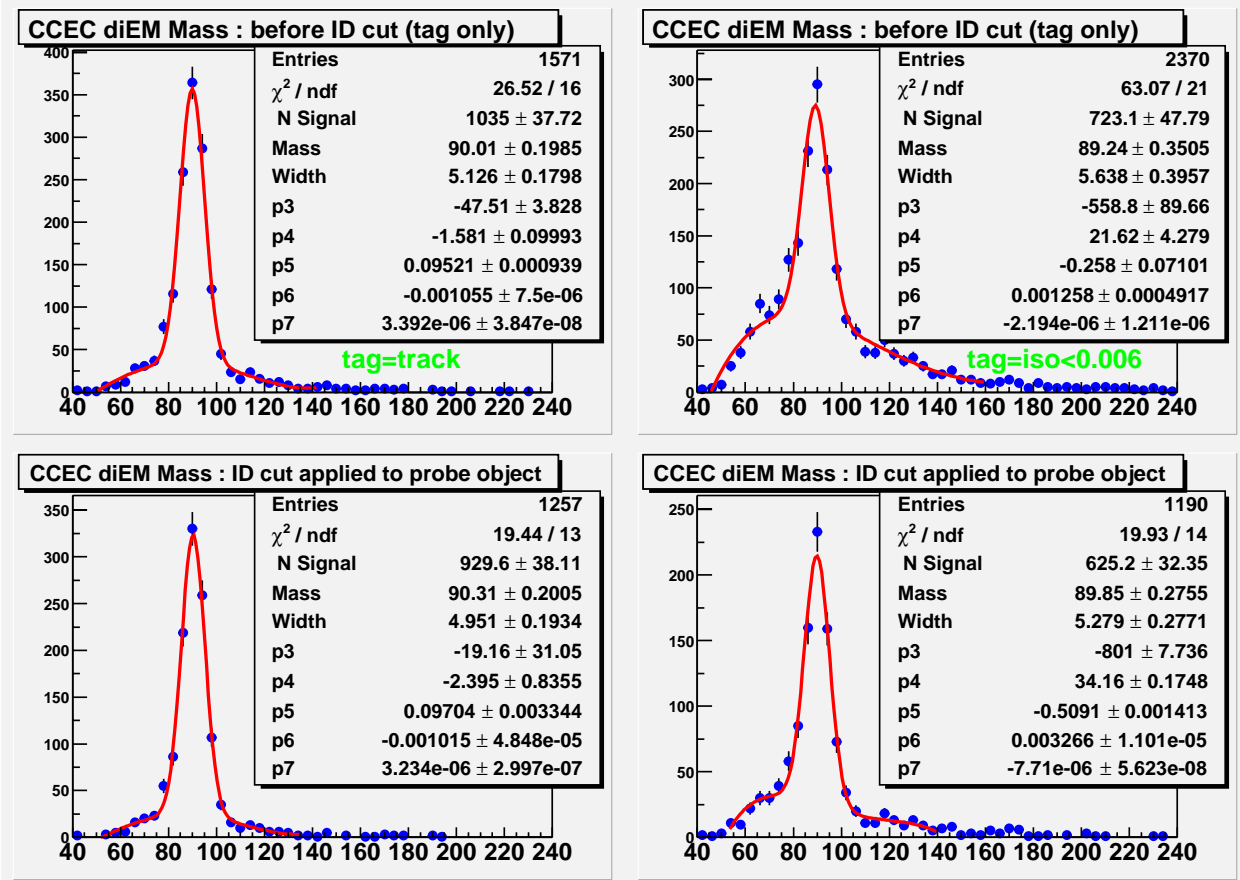

Figure 5.9: CCEC Mass distributions for measurement of $\epsilon(E C)$. Left column corresponds to the case when the tag object is required to have a track match, whereas in the right column calorimeter based cut $($ Isolation < 0.006$)$ is applied to the tag object. Top plots show the denominator mass distributions, i.e. when the cut is applied on the tag object only. Bottom plots show the numerator mass distributions, i.e. when the (EM) ID cut is applied to the probe object.

$\epsilon(C C)=80.7 \pm 1.1($ stat $) \pm 1.3($ syst $) \% ;$

$\epsilon(E C)=88.1 \pm 0.9($ stat $) \pm 1.7($ syst $) \%$.

The efficiencies are found to be $65.1 \pm 2.7 \%$ for CCCC and $77.6 \pm 3.4 \%$ for CCEC.

\subsection{Track Veto Efficiency}

We do not have a source of high $p_{T}$ photons in the data and therefore need to rely on the Monte

Carlo to estimate the track veto efficiency for $h \rightarrow \gamma \gamma$ events (events with at least one matching track are vetoed).

Tables 5.1 and 5.2 show the CCCC and CCEC track veto efficiencies (per $h \rightarrow \gamma \gamma$ event) for 60, 70, 80, 90, 100, $120 \mathrm{GeV}$ Higgs mass points. The inefficiency is mainly due to the photon conversions in the material in front of the fiber tracker. 


\begin{tabular}{ccc}
\hline$M_{\mathrm{h}}$ & CCCC & CCEC \\
\hline 60 & $94.8 \pm 0.7 \%$ & $93.2 \pm 1.8 \%$ \\
70 & $94.4 \pm 0.7 \%$ & $91.1 \pm 1.7 \%$ \\
80 & $95.6 \pm 0.6 \%$ & $92.1 \pm 1.5 \%$ \\
90 & $94.9 \pm 0.6 \%$ & $93.5 \pm 1.3 \%$ \\
100 & $94.3 \pm 0.7 \%$ & $94.6 \pm 1.2 \%$ \\
120 & $94.9 \pm 0.6 \%$ & $94.1 \pm 1.1 \%$ \\
\hline
\end{tabular}

Table 5.1: Track veto efficiency (per $h \rightarrow \gamma \gamma$ event) for the Fermiophobic Higgs.

\begin{tabular}{ccc}
\hline$M_{\mathrm{h}}$ & CCCC & CCEC \\
\hline 60 & $96.0 \pm 0.5 \%$ & $93.9 \pm 1.5 \%$ \\
70 & $94.7 \pm 0.5 \%$ & $91.7 \pm 1.3 \%$ \\
80 & $94.8 \pm 0.6 \%$ & $92.2 \pm 1.2 \%$ \\
90 & $95.3 \pm 0.5 \%$ & $91.7 \pm 1.1 \%$ \\
100 & $94.1 \pm 0.7 \%$ & $94.6 \pm 1.0 \%$ \\
120 & $94.8 \pm 0.6 \%$ & $93.8 \pm 0.9 \%$ \\
\hline
\end{tabular}

Table 5.2: Track veto efficiency (per $h \rightarrow \gamma \gamma$ event) for the Topcolor Higgs.

\section{$5.7 \quad h \rightarrow \gamma \gamma$ Width}

Two sources contribute to the width of the $h \rightarrow \gamma \gamma$ invariant mass peak: the natural width of the Higgs (Breit-Wigner) and photon energy resolution (Gaussian) in the calorimeter, the latter being the dominating source. Figures 5.10, 5.11, 5.12, and 5.13 show fitted $h \rightarrow \gamma \gamma$ MC mass peak for Fermiophobic Higgs (CCCC), Fermiophobic Higgs (CCEC), Topcolor Higgs (CCCC), and Topcolor Higgs (CCEC), respectively. 

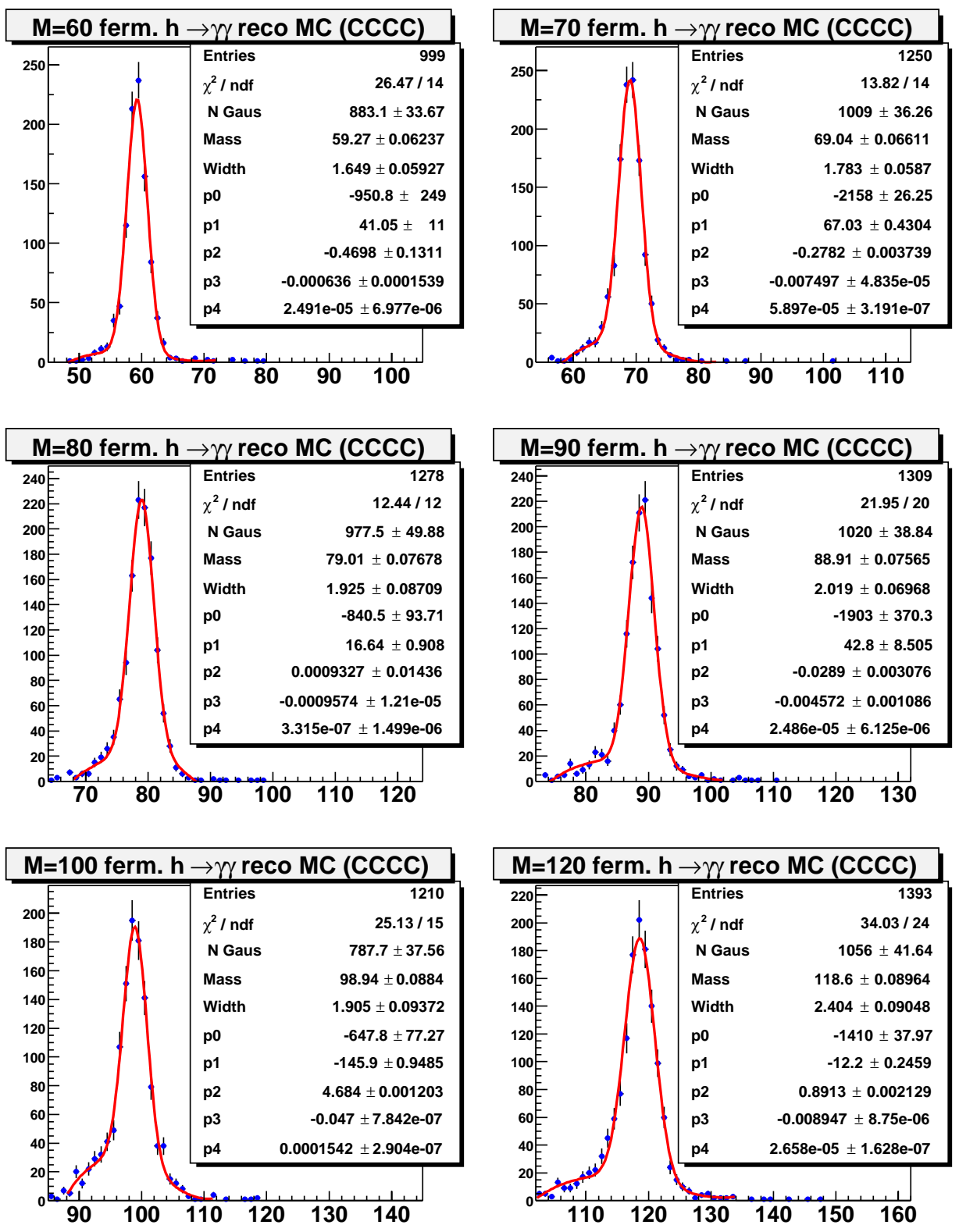

Figure 5.10: Fermiophobic $h \rightarrow \gamma \gamma$ mass peak in CCCC in reconstructed MC. 

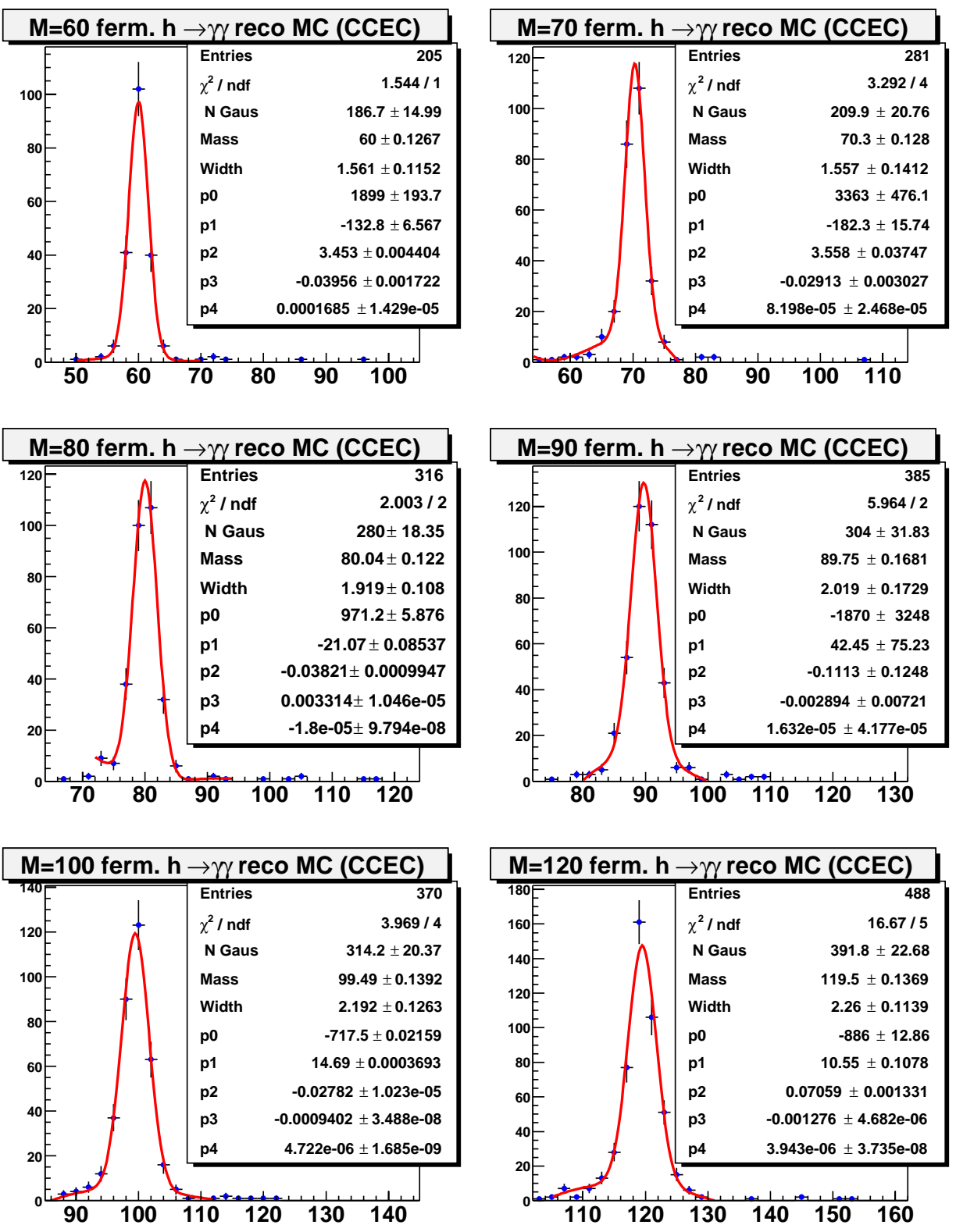

Figure 5.11: Fermiophobic $h \rightarrow \gamma \gamma$ mass peak in CCEC in reconstructed MC. 

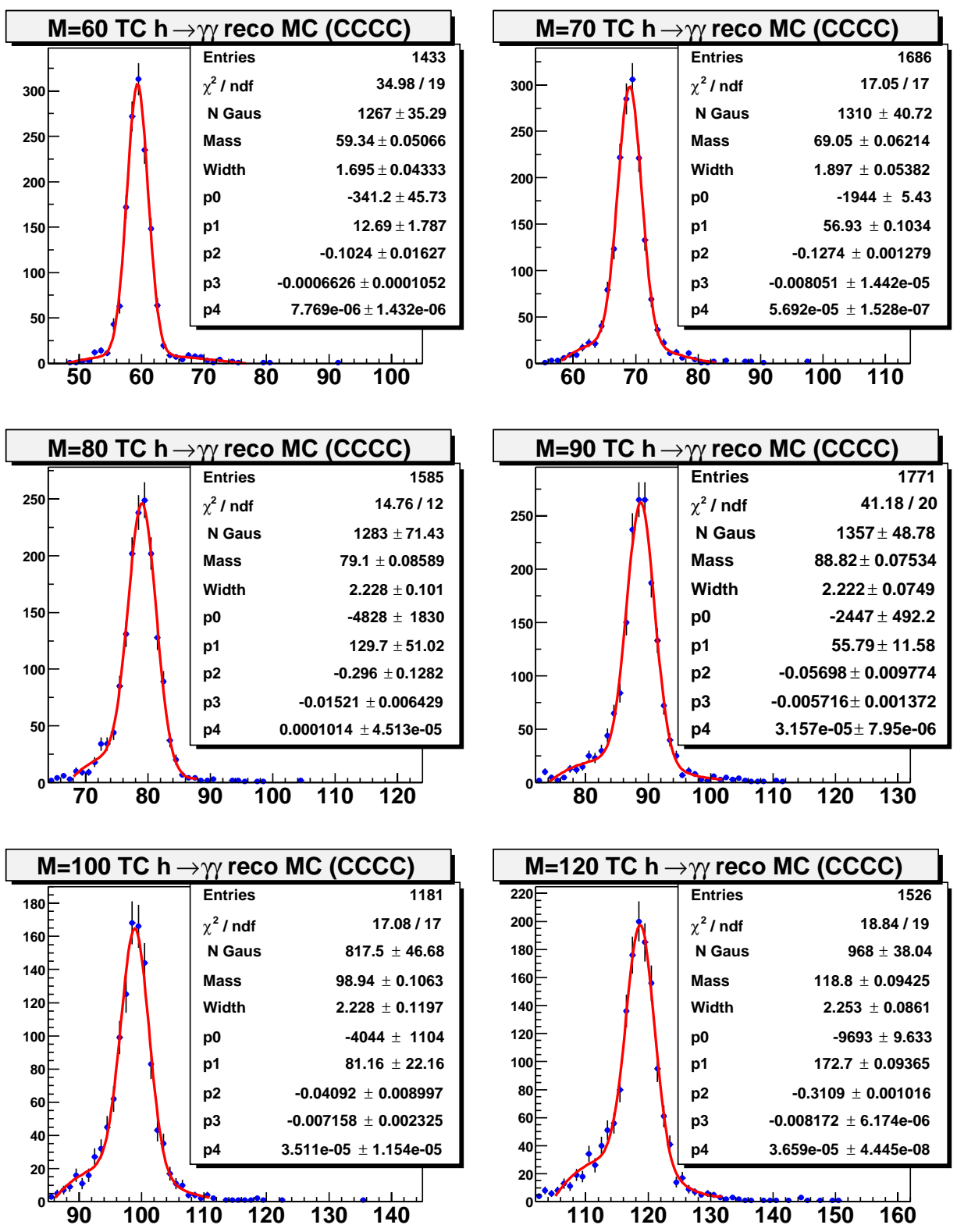

Figure 5.12: Topcolor $h \rightarrow \gamma \gamma$ mass peak in CCCC in reconstructed MC.

Following prescription of [35] for a Gaussian resonanse we derive the optimal mass window based on the Monte Carlo. In order to apply this to the data spectrum we need first to take into account the difference in energy resolution (and hence in the width) between the data and MC. This is done using $Z \rightarrow e e$ events. MC and data $Z \rightarrow e e$ distributions for CCCC are shown in Figs. 5.14 and 5.15, respectively. Figures 5.16 and 5.17 show same distributions for CCEC case. 

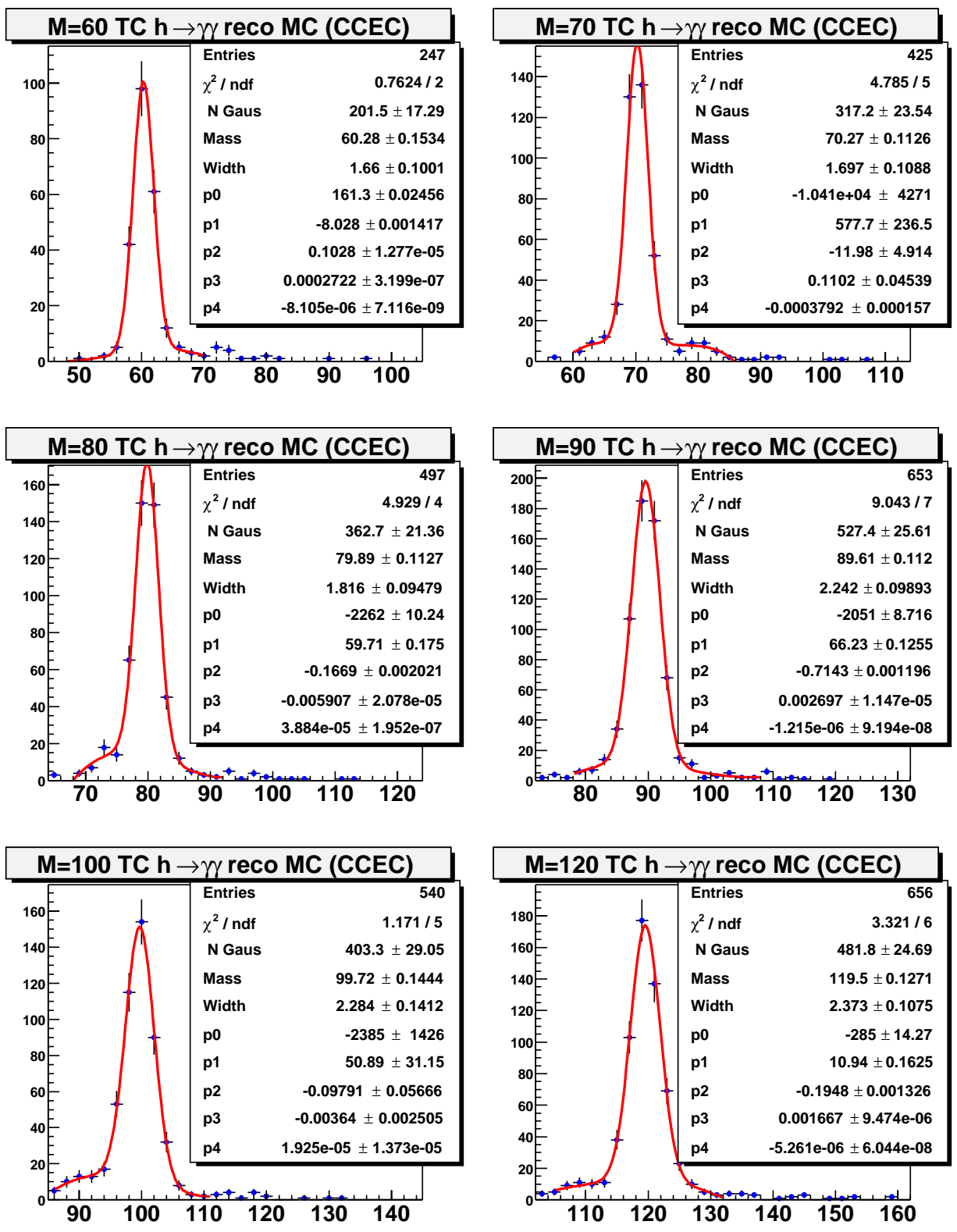

Figure 5.13: Topcolor $h \rightarrow \gamma \gamma$ mass peak in reconstructed MC. 


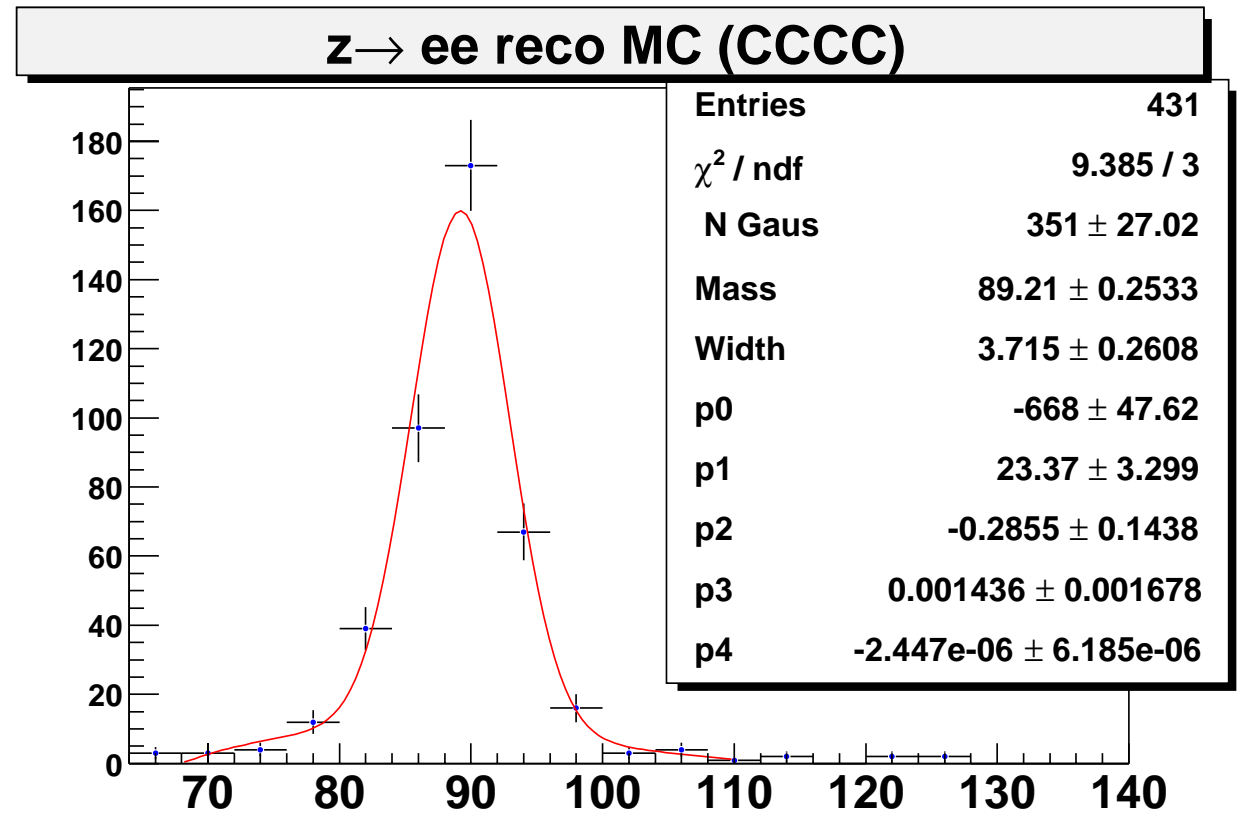

Figure 5.14: $Z \rightarrow e e$ mass peak in CCCC for reconstructed MC. We use only calorimetric energy and direction measurements to calculate the invariant mass.

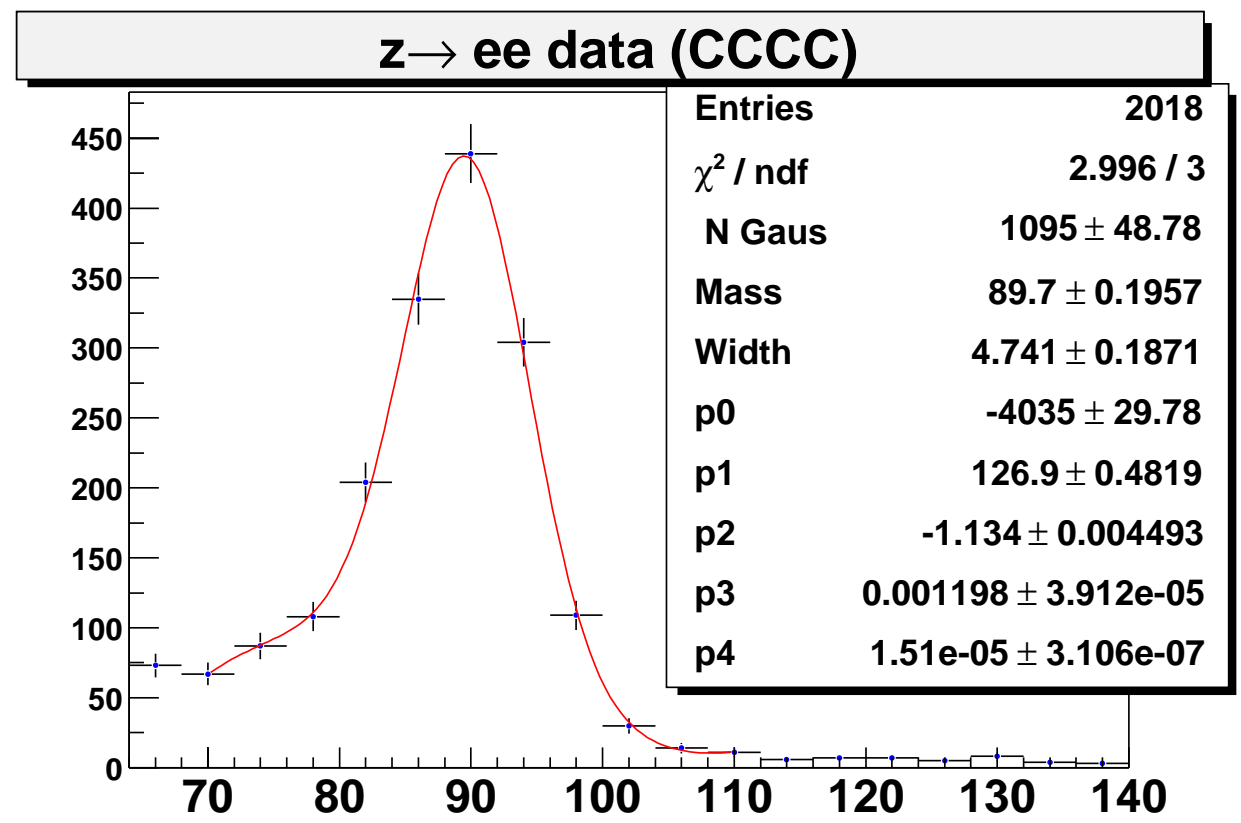

Figure 5.15: $Z \rightarrow e e$ mass peak in CCCC for the data. We use only calorimetric energy and direction measurements to calculate the invariant mass. 


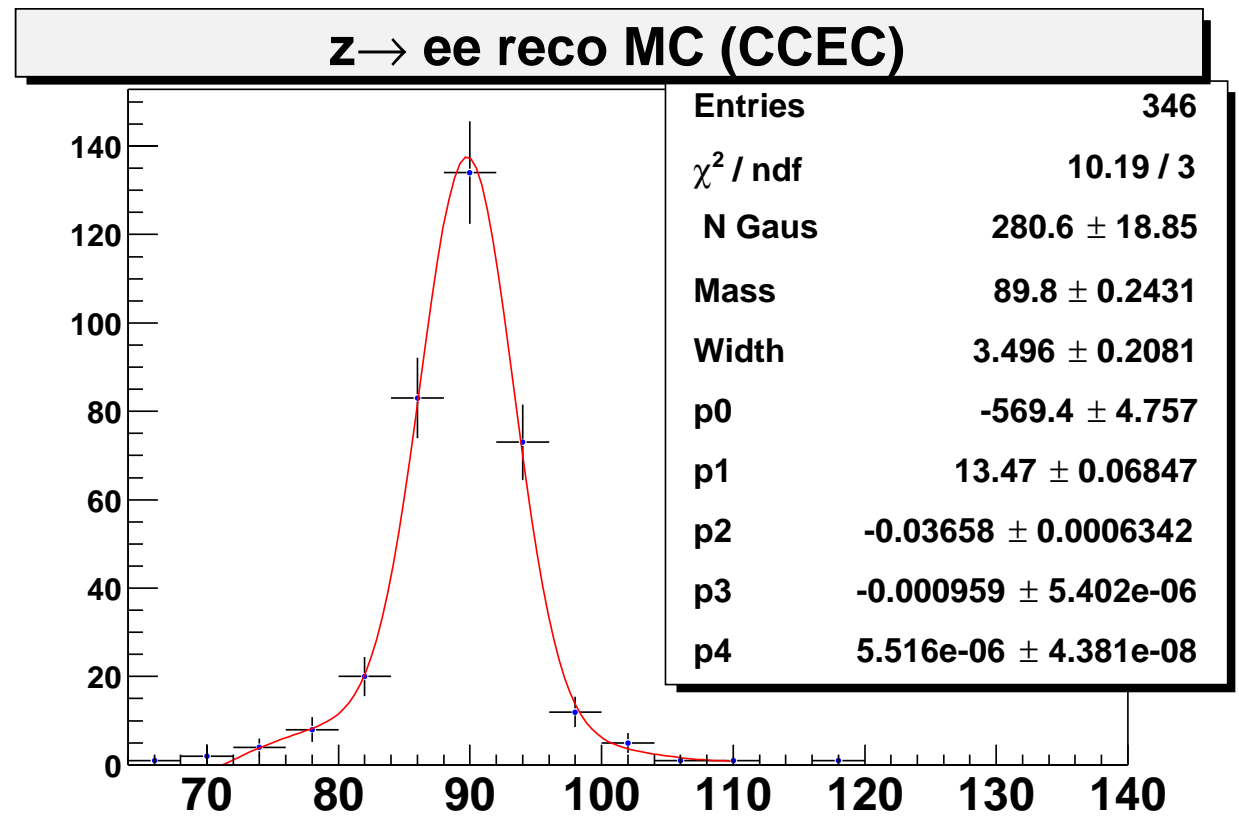

Figure 5.16: $Z \rightarrow e e$ mass peak in CCEC for reconstructed MC. We use only calorimetric energy and direction measurements to calculate the invariant mass.

The width of the $\mathrm{Z}$ peak derived from the Gaussian fit is:

$\sigma(z \rightarrow$ ee data $)=4.74 \pm 0.19 \mathrm{GeV}(\mathrm{CCCC})$

$\sigma(z \rightarrow e e M C)=3.72 \pm 0.26 \mathrm{GeV}(\mathrm{CCCC})$

$\sigma(z \rightarrow$ ee data $)=4.26 \pm 0.21 \mathrm{GeV}(\mathrm{CCEC})$

$\sigma(z \rightarrow e e M C)=3.50 \pm 0.21 \mathrm{GeV}(\mathrm{CCEC})$

Then $M C \rightarrow$ data correction factor, $k$, is calculated as a ratio between the widths in the data and MC:

$k(M C \rightarrow$ data $)=1.28 \pm 0.10(\mathrm{CCCC})$

$k(M C \rightarrow$ data $)=1.22 \pm 0.09(\mathrm{CCEC})$.

\subsection{Acceptance}

We separate signal acceptance into two components: 


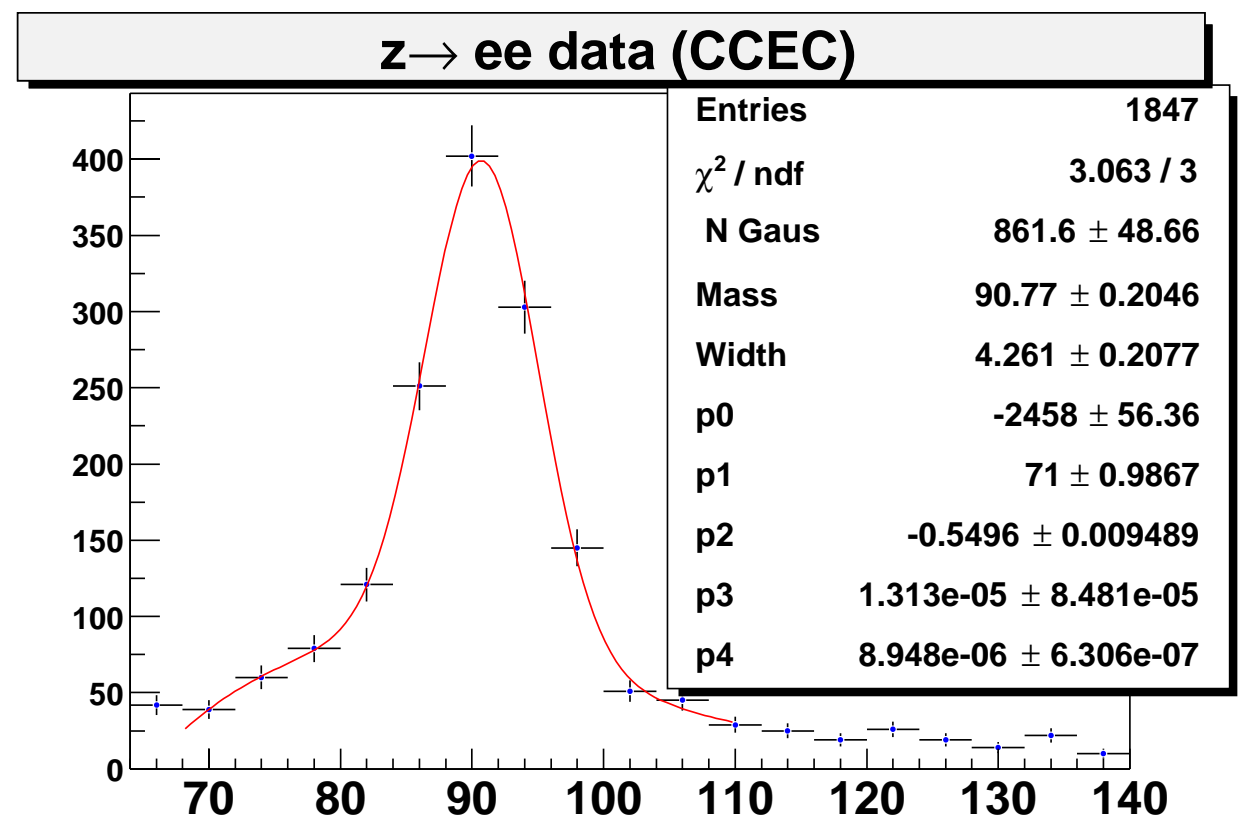

Figure 5.17: $Z \rightarrow e e$ mass peak in CCEC for the data. We use only calorimetric energy and direction measurements to calculate the invariant mass.

1. The acceptance due to the fiducial and $p_{T}$ cuts;

2. The acceptance due to the mass window cut.

\subsubsection{Mass Window Cut Acceptance}

Mass window cut acceptance is calculated using reconstructed MC events. Uncertainty in the mass window acceptance is estimated as half of the difference in acceptance for the mass window variation by $\pm 1 \sigma$ of the scale factor $k$.

Table 5.3 shows the CCCC and CCEC mass window acceptances for 60, 70, 80, 90, 100, $120 \mathrm{GeV}$ Higgs mass points for Fermiophobic Higgs. Table 5.4 shows the same for the Topcolor Higgs.

\subsubsection{Fiducial and $p_{T}$ Acceptance}

Fiducial and $p_{T}$ acceptance is calculated using reconstructed MC. Systematic uncertainty of this measurement comes mostly from the calorimeter energy resolution effects. We estimate it by smearing the generator level object energies. Generator-level information needs to be used since studying 


\begin{tabular}{ccc}
\hline$M_{\mathrm{h}}$ & CCCC & CCEC \\
\hline 60 & $78.7 \pm 2.3 \%$ & $81.5 \pm 1.5 \%$ \\
70 & $75.3 \pm 2.8 \%$ & $74.4 \pm 3.0 \%$ \\
80 & $72.5 \pm 3.1 \%$ & $75.3 \pm 3.2 \%$ \\
90 & $72.4 \pm 3.2 \%$ & $75.1 \pm 2.6 \%$ \\
100 & $66.9 \pm 2.4 \%$ & $75.7 \pm 1.9 \%$ \\
120 & $70.6 \pm 3.4 \%$ & $74.8 \pm 2.2 \%$ \\
\hline
\end{tabular}

Table 5.3: Mass window acceptance for Fermiophobic Higgs

\begin{tabular}{ccc}
\hline$M_{\mathrm{h}}$ & CCCC & CCEC \\
\hline 60 & $77.7 \pm 2.9 \%$ & $76.5 \pm 2.2 \%$ \\
70 & $72.3 \pm 2.8 \%$ & $69.4 \pm 2.5 \%$ \\
80 & $74.9 \pm 3.5 \%$ & $70.0 \pm 3.6 \%$ \\
90 & $71.0 \pm 2.8 \%$ & $75.3 \pm 2.3 \%$ \\
100 & $68.3 \pm 3.1 \%$ & $70.4 \pm 2.5 \%$ \\
120 & $64.3 \pm 3.2 \%$ & $68.9 \pm 2.8 \%$ \\
\hline
\end{tabular}

Table 5.4: Mass window acceptance for Topcolor Higgs

resolution effects at the reconstructed level would involve convolution of two Gaussians (from the original calorimeter energy resolution as described by MC and the additional smearing).

On the other hand, generator-level information alone can not be used as it gives artificially higher acceptance, since the effects of the calorimeter $\phi$-module boundaries and efficiency losses due to jet overlapping with EM objects are not accounted for. Therefore, we calculate acceptance using reconstructed-level information, estimate relative systematic uncertainties from the generator level, and apply them to the acceptances calculated from reconstructed MC.

First, the $Z \rightarrow e e$ MC generator level electron energy is smeared to obtain agreement with the data in the Gaussian contribution to the $\mathrm{Z}$ mass width that describes the calorimeter energy resolution. Smearing is done following [90]. A sampling calorimeter resolution is commonly expressed as

$$
\left(\frac{\sigma}{E}\right)^{2}=C^{2}+\frac{S^{2}}{E}+\frac{N^{2}}{E^{2}},
$$

where $E$ is particle energy, 
$C$ is a constant contribution from systematic errors such as remaining channel-to-channel variation in calorimeter gain;

$S$ is due to the statistical error in shower sampling;

$N$ represents energy-independent contributions, such as electronic and uranium noise.

Since the sampling term is dominant [22] we reduce equation $(5.11)$ to $\sigma=S \times \sqrt{E}$. Figure 5.18 shows fitted Z mass peak in the CCCC events for smeared MC, while Fig. 5.19 shows the corresponding $Z$ mass peak in the data.

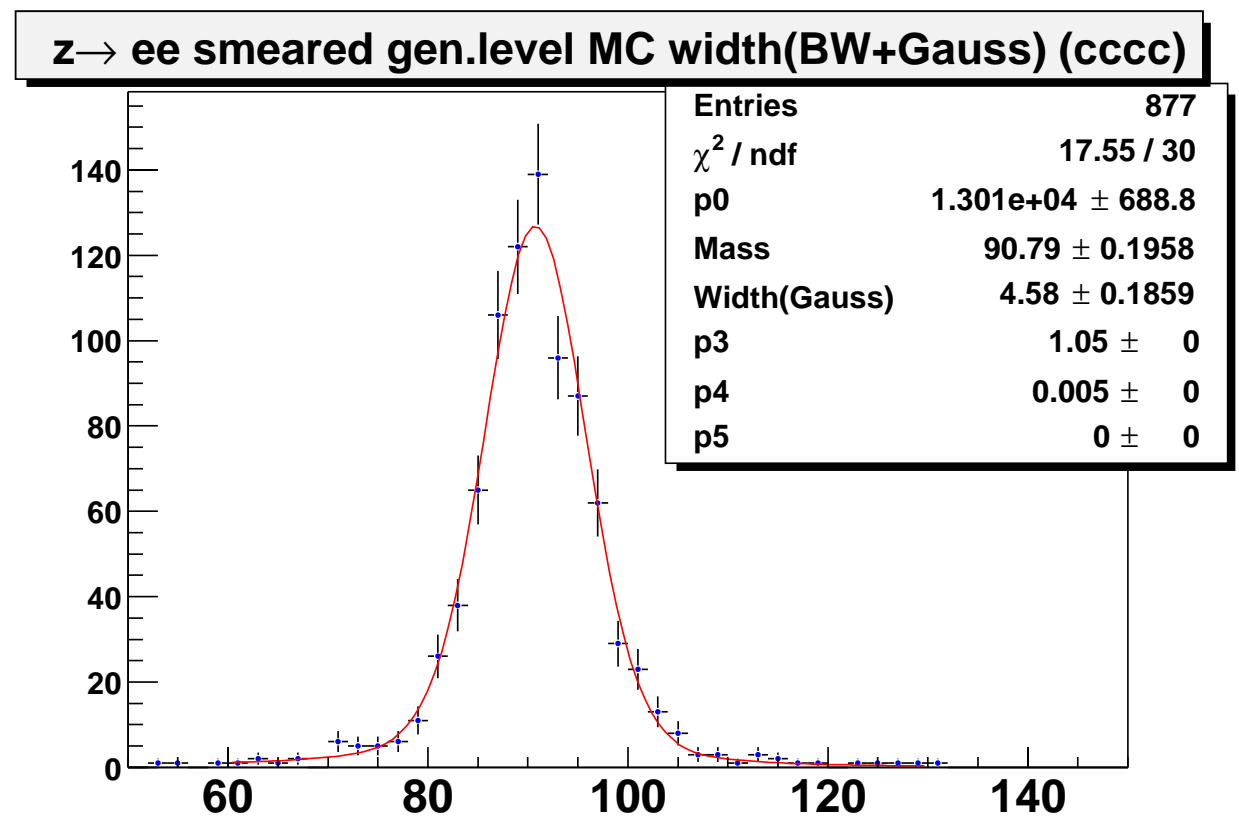

Figure 5.18: $Z \rightarrow$ ee mass peak in the CCCC events for smeared generator-level MC.

A convolution of the Breit-Wigner and Gaussian functions is used to fit the $Z$ mass peak. Then the smearing coefficients are adjusted so that the Gaussian part of smeared MC width matches that in the data. Figures 5.20 and 5.21 show the same MC and data distributions for the CCEC events. Smearing coefficients $S(C C)$ and $S(E C)$ are found to be:

$S(C C)=0.454$ 


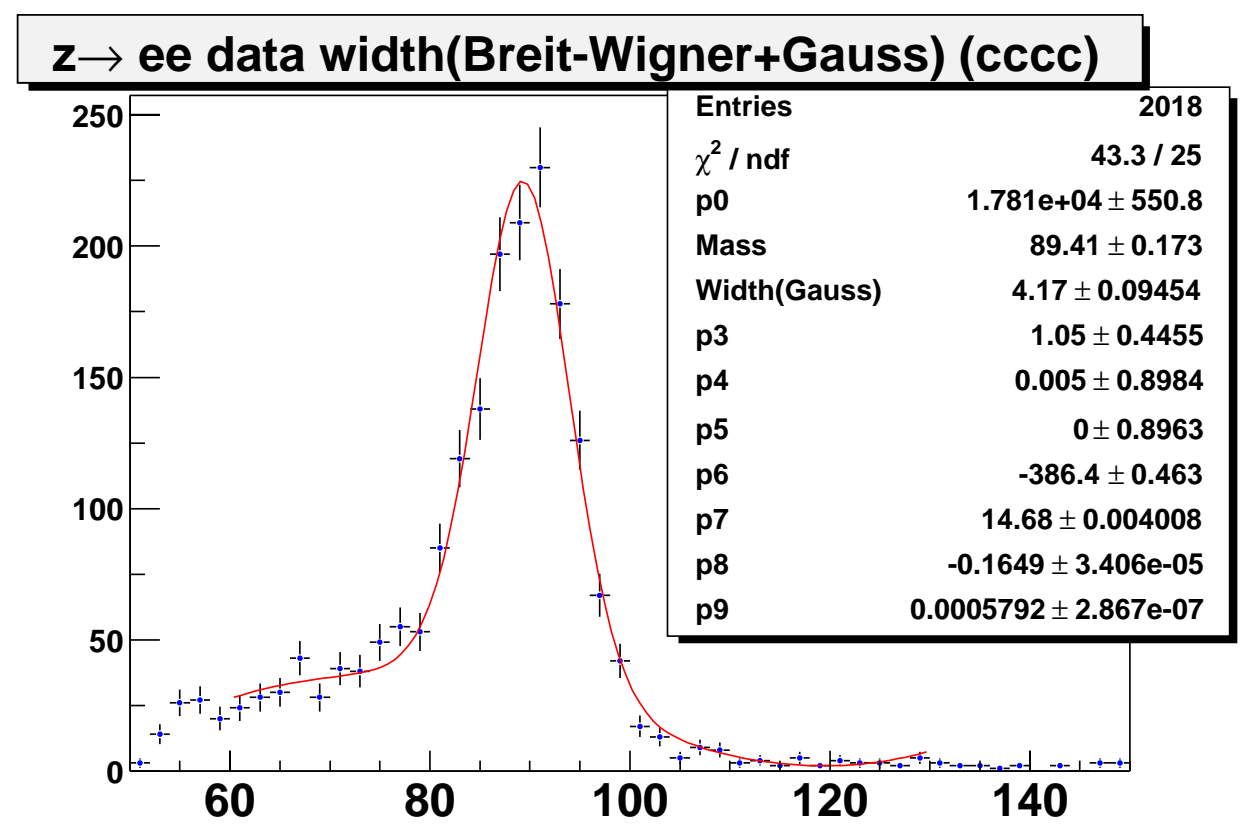

Figure 5.19: $Z \rightarrow e e$ mass peak in the CCCC events for the data.

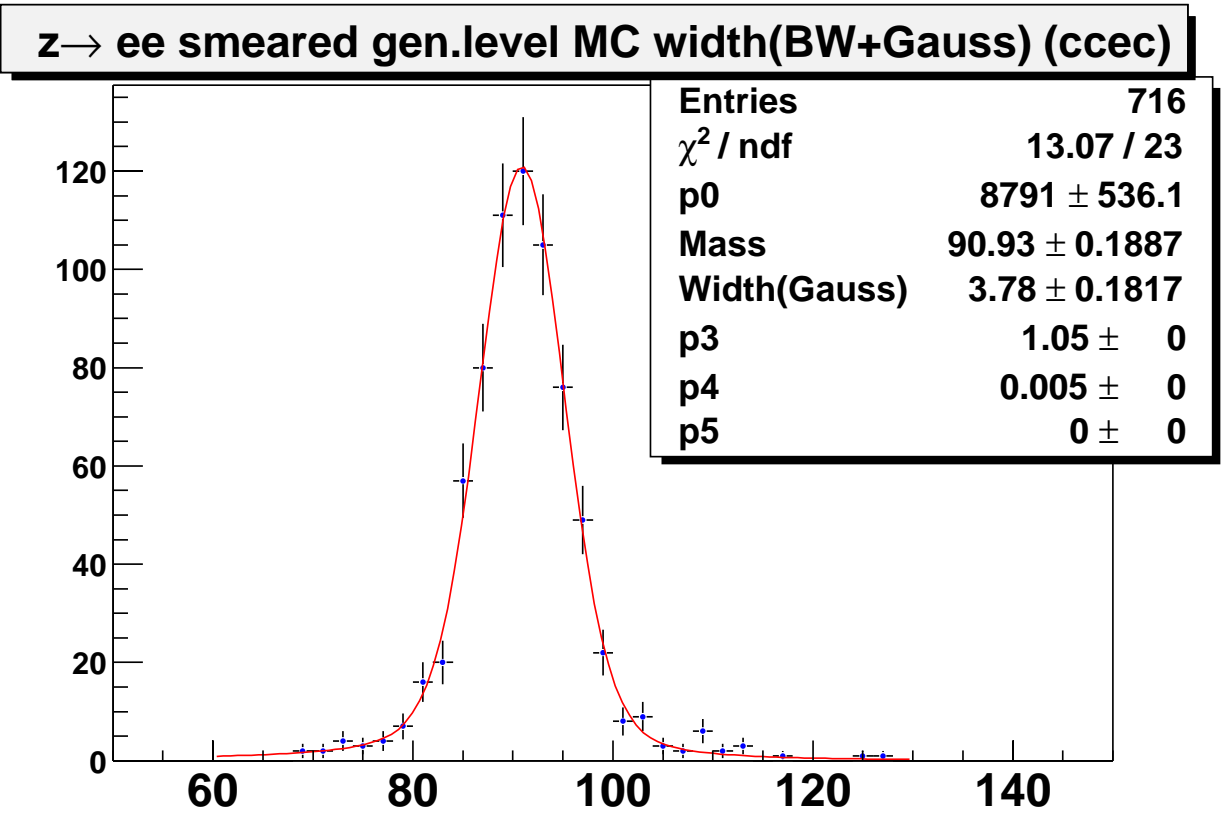

Figure 5.20: $Z \rightarrow e e$ mass peak in the CCEC events for smeared generator-level MC. 


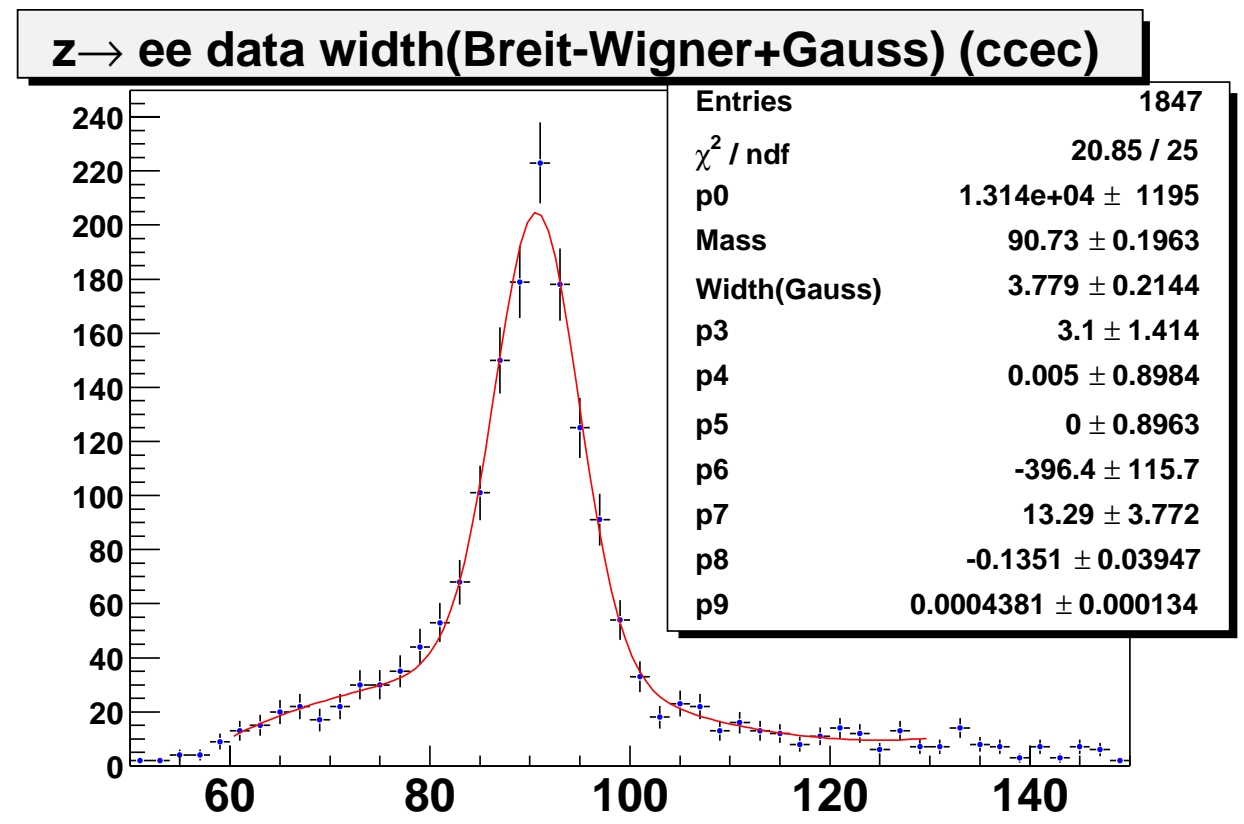

Figure 5.21: $Z \rightarrow e e$ mass peak in the CCEC events for the data.

$$
\begin{aligned}
& S_{+}(C C)=0.462 \\
& S_{-}(C C)=0.442 \\
& S(E C)=0.44 \\
& S_{+}(E C)=0.5 \\
& S_{-}(E C)=0.38
\end{aligned}
$$

where $S_{+}$and $S_{-}$coefficients correspond to the oversmeared and undersmeared energies to match the mass peak width $\pm 1 \sigma$ in $Z \rightarrow e e$ data.

Then the smearing is applied to the $h \rightarrow \gamma \gamma$ generator-level MC and the acceptance is calculated. The uncertainty is estimated as half of the difference in acceptance calculated with the values of smearing coefficients $S_{+}$and $S_{-}$.

Table 5.5 shows the acceptance for 60, 70, 80, 90, 100, $120 \mathrm{GeV}$ Fermiophobic Higgs mass points. Table 5.6 shows that for the Topcolor Higgs mass points. 


\begin{tabular}{ccc}
\hline$M_{\mathrm{h}}, \mathrm{GeV}$ & $\mathrm{CCCC}$ & $\mathrm{CCEC}$ \\
\hline 60 & $13.6 \pm 0.4 \%$ & $2.8 \pm 0.2 \%$ \\
70 & $19.0 \pm 0.4 \%$ & $4.2 \pm 0.2 \%$ \\
80 & $24.8 \pm 0.5 \%$ & $6.4 \pm 0.3 \%$ \\
90 & $28.2 \pm 0.6 \%$ & $8.4 \pm 0.4 \%$ \\
100 & $31.3 \pm 0.7 \%$ & $9.9 \pm 0.4 \%$ \\
120 & $34.6 \pm 0.7 \%$ & $12.9 \pm 0.5 \%$ \\
\hline
\end{tabular}

Table 5.5: Fiducial and $p_{T}$ acceptance for Fermiophobic Higgs.

\begin{tabular}{ccc}
\hline$M_{\mathrm{h}}, \mathrm{GeV}$ & $\mathrm{CCCC}$ & $\mathrm{CCEC}$ \\
\hline 60 & $13.1 \pm 0.3 \%$ & $2.3 \pm 0.1 \%$ \\
70 & $20.2 \pm 0.4 \%$ & $4.9 \pm 0.2 \%$ \\
80 & $25.5 \pm 0.5 \%$ & $8.3 \pm 0.3 \%$ \\
90 & $28.7 \pm 0.5 \%$ & $11.0 \pm 0.4 \%$ \\
100 & $29.0 \pm 0.6 \%$ & $13.5 \pm 0.5 \%$ \\
120 & $32.8 \pm 0.6 \%$ & $14.9 \pm 0.5 \%$ \\
\hline
\end{tabular}

Table 5.6: Fiducial and $p_{T}$ acceptance for Topcolor Higgs.

\subsection{Event Counts}

Using the CCCC subsample for each Higgs mass point, we count the number of $\gamma \gamma$ events in an optimal mass window and estimate background. Resulting event counts are shown in Table 5.7 for the Fermiophobic Higgs and Topcolor Higgs. 


\begin{tabular}{cccc}
\hline$M_{\mathrm{h}}, \mathrm{GeV}$ & Window size, GeV & Data & Background \\
\hline \multicolumn{4}{c}{ Fermiophobic Higgs } \\
\hline 60 & $4.66 \pm 0.37$ & 56 & $48.0 \pm 5.6$ \\
70 & $5.02 \pm 0.41$ & 72 & $57.8 \pm 6.9$ \\
80 & $5.42 \pm 0.43$ & 68 & $60.0 \pm 9.2$ \\
90 & $5.70 \pm 0.46$ & 107 & $93.7 \pm 20.1$ \\
100 & $5.38 \pm 0.43$ & 30 & $30.5 \pm 5.0$ \\
120 & $6.78 \pm 0.54$ & 7 & $9.7 \pm 1.8$ \\
\hline \multicolumn{4}{c}{ Topcolor Higgs } \\
70 & $4.78 \pm 0.38$ & 57 & $49.8 \pm 5.8$ \\
80 & $5.34 \pm 0.43$ & 76 & $61.0 \pm 7.2$ \\
90 & $6.28 \pm 0.50$ & 78 & $71.4 \pm 10.4$ \\
100 & $6.26 \pm 0.51$ & 113 & $99.0 \pm 21.1$ \\
120 & $6.28 \pm 0.50$ & 33 & $33.6 \pm 5.6$ \\
\hline
\end{tabular}

Table 5.7: CCCC event counts

\subsection{Analysis Optimization}

\subsubsection{Outline of Optimization}

Our next step is to optimize the analysis so that the sensitivity is improved. We explore several options :

- include jets in the analysis and cut on jet multiplicity;

- use the following kinematic variables:

1. $p_{T}$ of the diphoton system $p_{T}^{\gamma \gamma}$;

2. $\eta-\phi$ separation between the two photons $d R^{\gamma \gamma}(\eta, \phi)$;

3. diphoton energy asymmetery $(E 1-E 2) /(E 1+E 2)$;

4. $\left|\cos \theta^{*}\right|\left(\theta^{*}\right.$ is the angle between the direction of the photons in the $\gamma \gamma$ rest frame and the diphoton system momentum in the lab frame).

- study correlations between the variables and try to improve sensitivity by combining them in a Neural Net. 
Finally, once the optimization cuts are established, we add the CCEC subsample (by combining CCCC and CCEC likelihoods) for possible improvement in cross-section limits.

\subsection{2 $p_{T}^{\gamma \gamma}$ and Jet Multiplicity}

Our initial goal is to answer two questions :

1. What is the optimal $p_{T}^{\gamma \gamma}$ cut?

2. Is it beneficial to include jet selection in the analysis?

We expect an improvement from cutting on the jet multiplicity due to the fact that the signal is more likely to have extra jets (especially the Fermiophobic Higgs signal) than the background. The downside of including jets is introducing extra systematics due to the Jet Energy Scale (JES) uncertainties and reducing the data sample as we need to veto bad Jet/MET runs (18\% of the sample).

Jet multiplicity is expected to be correlated with $p_{T}^{\gamma \gamma}$. Since Higgs $p_{T}\left(p_{T}^{\gamma \gamma}\right)$ is balanced by the $p_{T}$ of the jets that come from the $W / Z$ decays into quark final states quarks, the higher the Higgs $p_{T}$ the more jets above certain threshold we expect. Therefore we determine optimal $p_{T}^{\gamma \gamma}$ cut for the two cases : 1) no jets are used in the analysis; 2) jet multiplicity cut is applied. We perform these studies for the case of Fermiophobic Higgs CCCC subsample.

Figure 5.22 shows the jet multiplicity distributions for the signal and the background for the six mass points.

We use jet energy scale JES-corrected jets [92] (jet cone radius $=0.7$ ). Background is estimated from data. The $Z \rightarrow e e$ background is extracted from the di-EM sample in the same way as it is done in the analysis (see Section 5.2.2). QCD background is calculated by selecting two objects that pass the Isolation and fail the H-matrix Photon selection criteria. Then the relative normalization is done using predicted individual background contributions for each mass point. Since jet multiplicity is a discrete variable we can easily see from Figure 5.22 that optimal choice is to require at least one jet in the event for any mass point. 

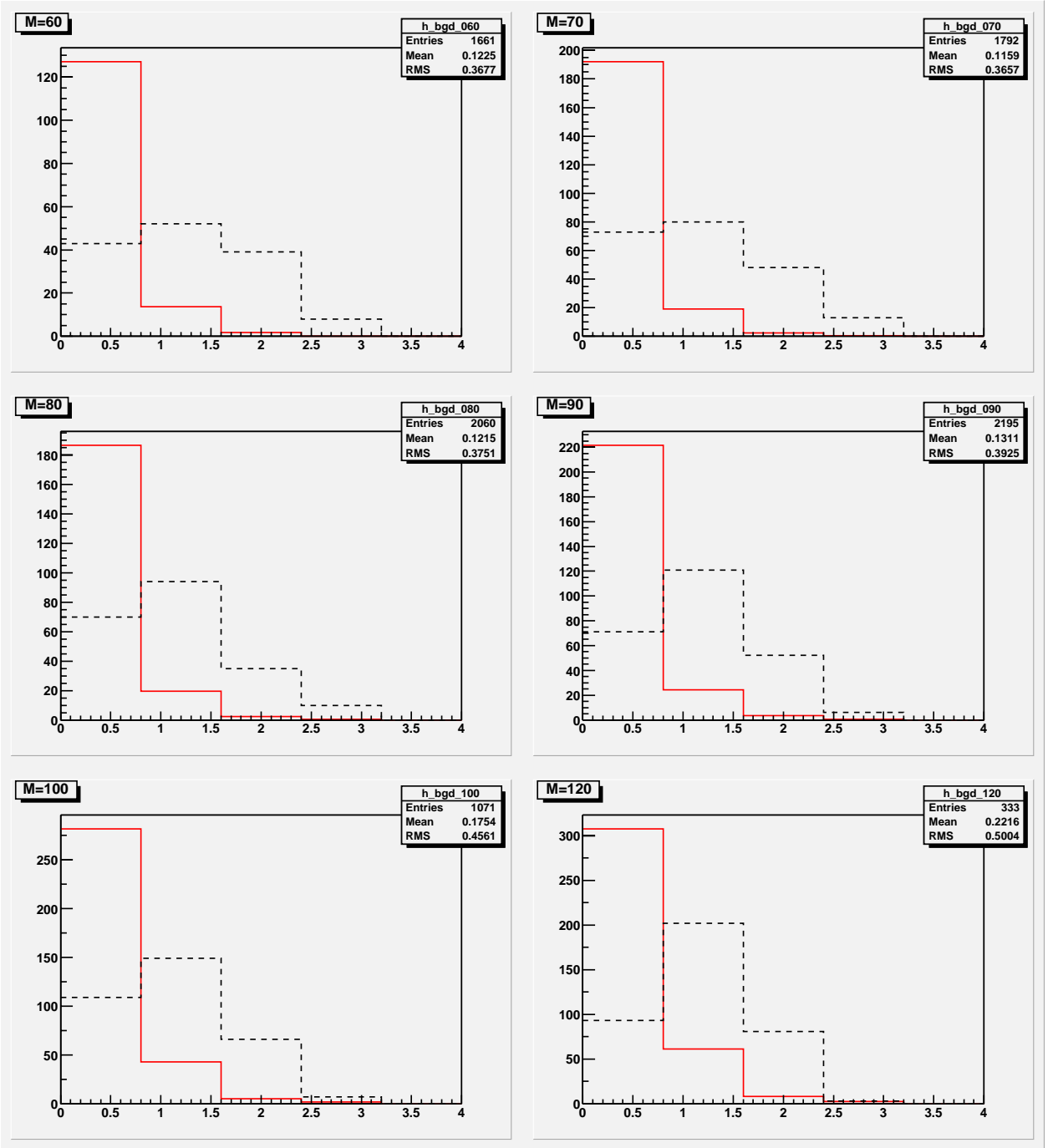

Figure 5.22: Jet multiplicity for the signal (dashed line) and the background (solid line). 
In addition to the jet multiplicity itself we are also interested in the systematics due to the error on JES correction. Relative uncertainty in jet $p_{T}$ due to the JES correction error is shown as a function of $\eta$ for data (Fig. 5.23) and M=120 GeV Higgs MC (Figure 5.24).

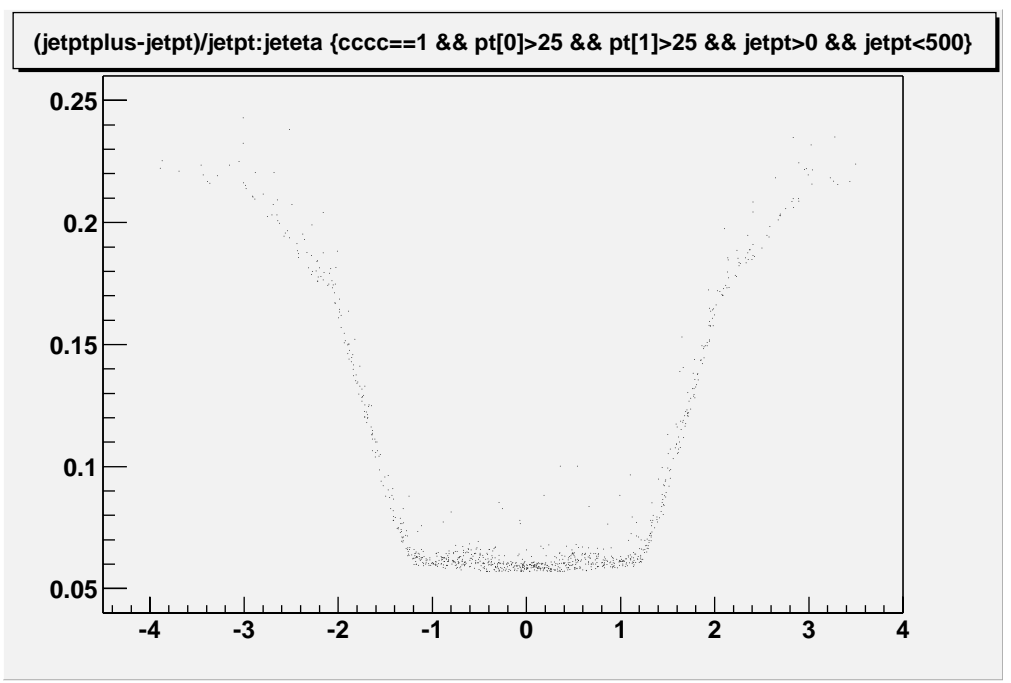

Figure 5.23: Relative uncertainty in jet $p_{T}$ due to JES error as a function of jet $\eta$ for $\mathrm{M}=120 \mathrm{GeV}$ Fermiophobic Higgs.

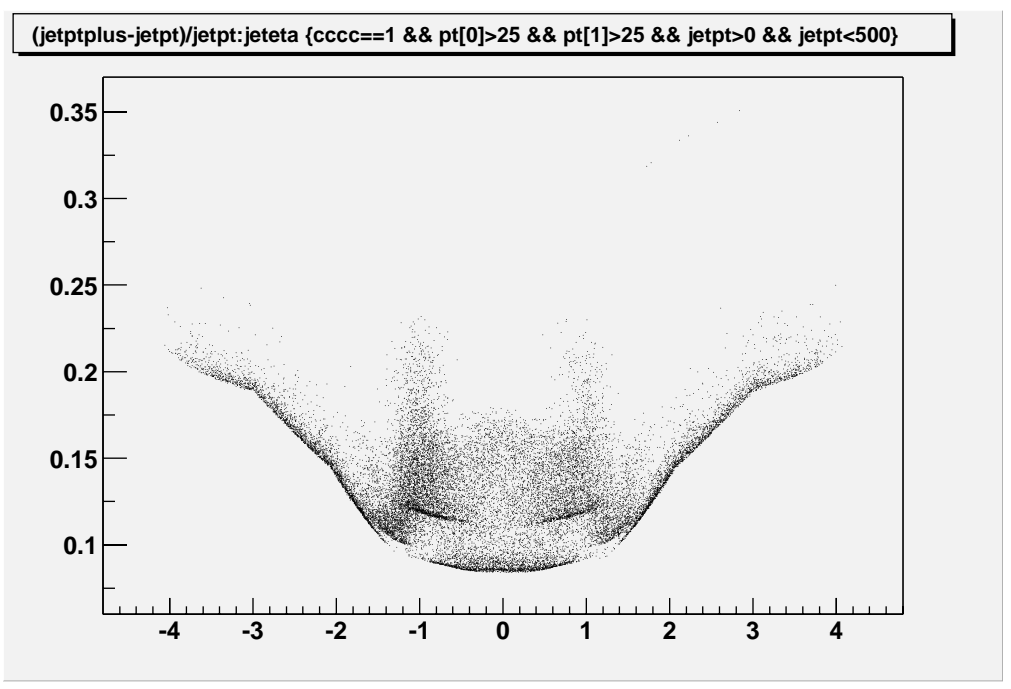

Figure 5.24: Relative uncertainty on the jet PT due to JES error as a function of jet $\eta$ for the di-EM data.

Jet multiplicity $\left(p_{T}>30 \mathrm{GeV}\right)$ distributions for JES-corrected jets and for under/over corrected by $\pm 1 \sigma$ jets are shown in Figure 5.25 for the Higgs MC and in Fig. 5.26 for data. 


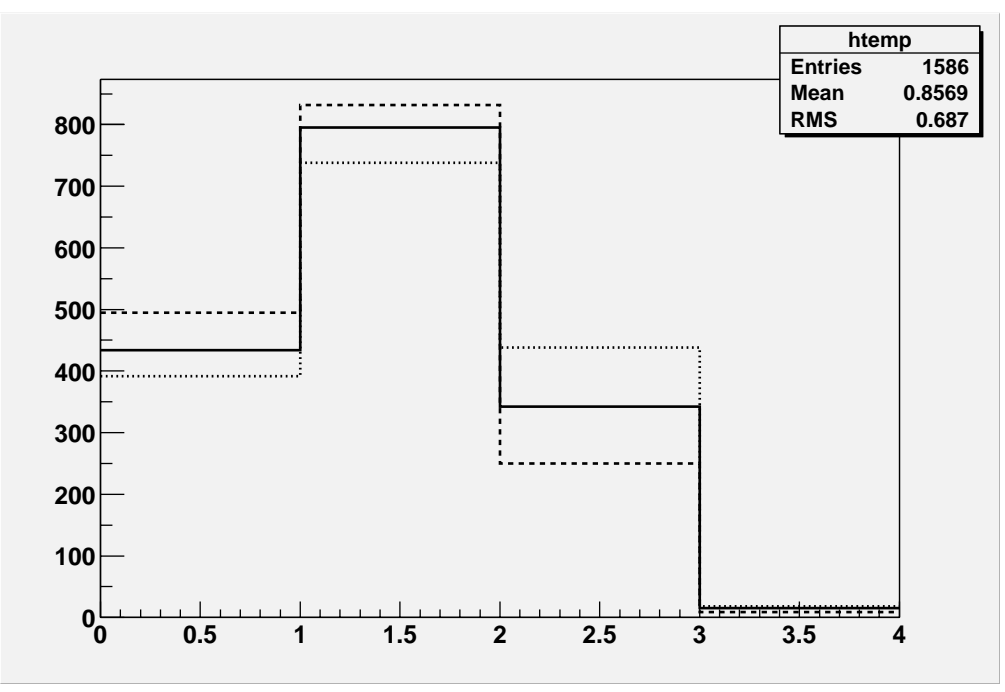

Figure 5.25: Jet multiplicity (number of jets with $p_{T}>30 \mathrm{GeV}$ ) for the $\mathrm{M}=120 \mathrm{GeV}$ Fermiophobic Higgs. Black line corresponds to JES corrected jets while dotted and dashed lines show $J E S+\sigma$ and $J E S-\sigma$ corrected jets respectively

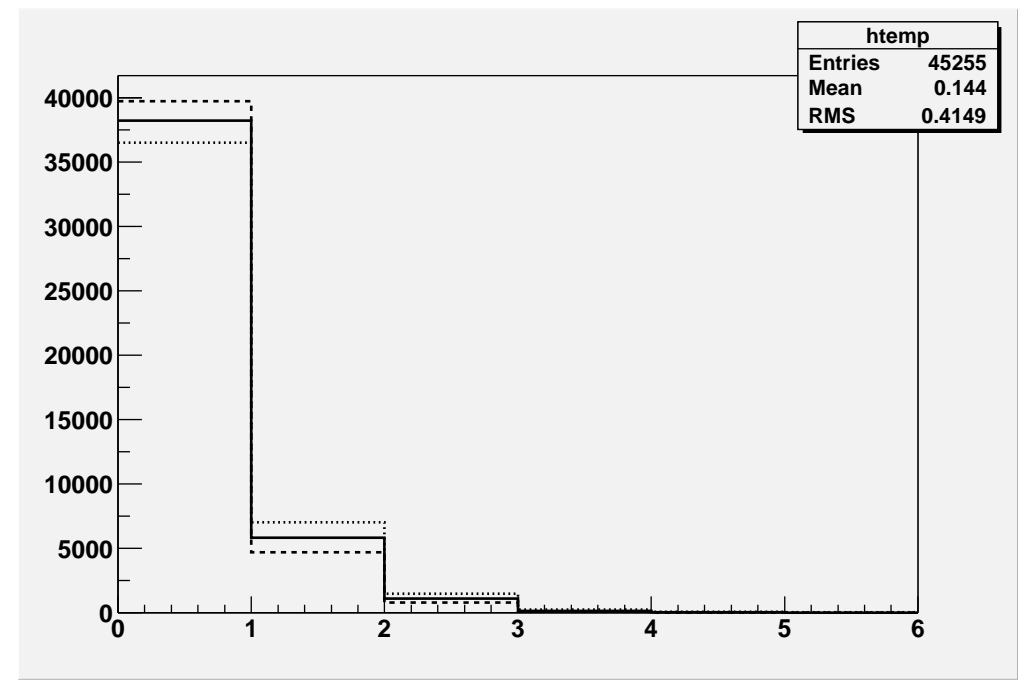

Figure 5.26: Jet multiplicity (number of jets with $p_{T}>30 \mathrm{GeV}$ )in data. Solid line corresponds to JES corrected jets while dotted and dashed line show $J E S+\sigma$ and $J E S-\sigma$ corrected jets respectively 
The systematic and statistical contributions to the JES correction uncertainty were added in quadrature in these plots. In the actual analysis we followed a more conservative approach and added the two contributions linearly.

Next, we look at $p_{T}^{\gamma \gamma}$ distributions for signal and background for the case when no jet cuts are applied (Figure 5.27) and when at least one jet $\left(p_{T}>30 \mathrm{GeV}\right)$ is required to be present in the event (Figure 5.28).
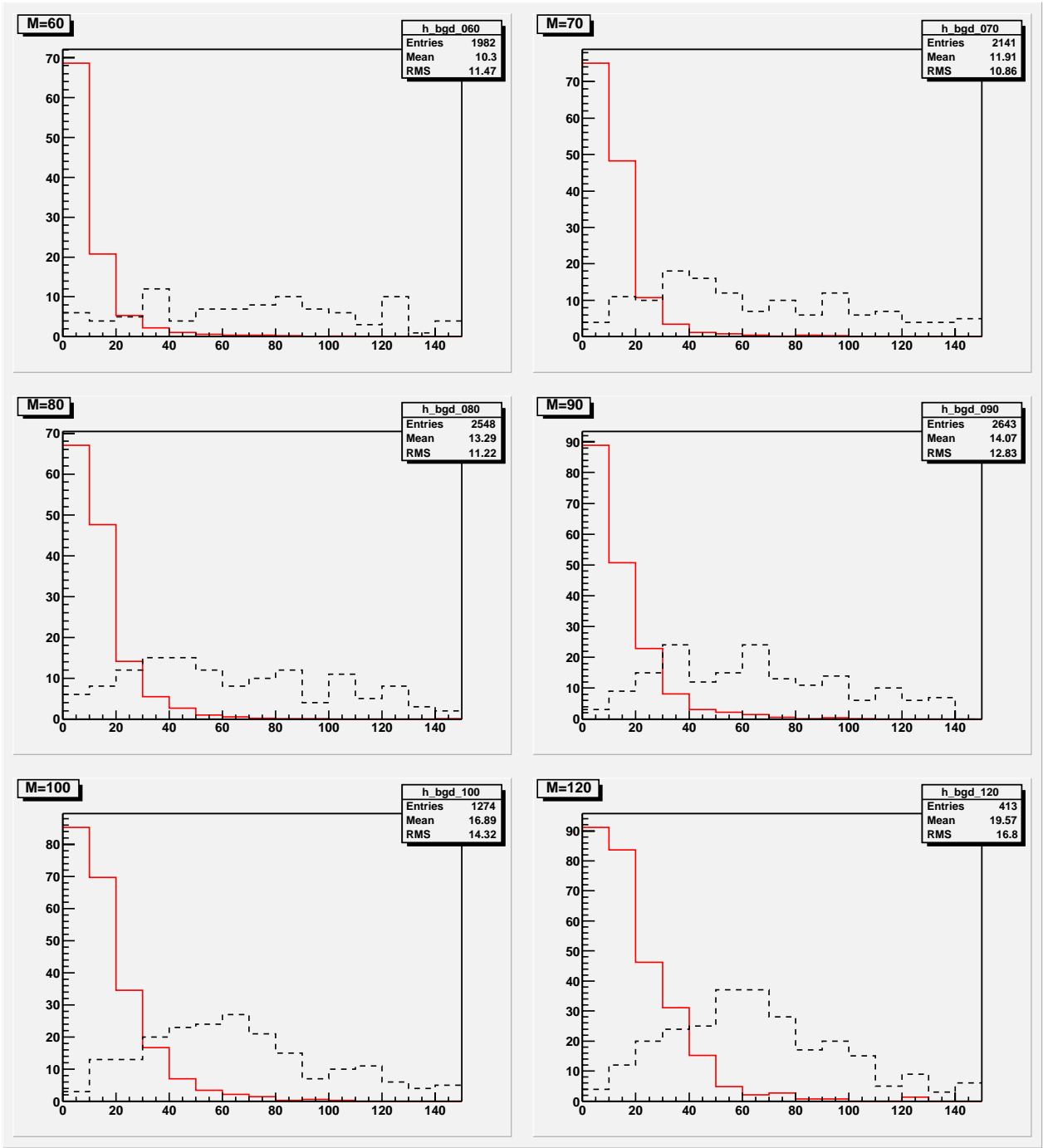

Figure 5.27: $p_{T}^{\gamma \gamma}$ distribution for signal (dashed line) and background (solid line). No jet multiplicity cut is applied.

Comparing these two figures we can see that the separation between the signal and the background in the latter is worse as some of the discrimination power is already contained in the jet 

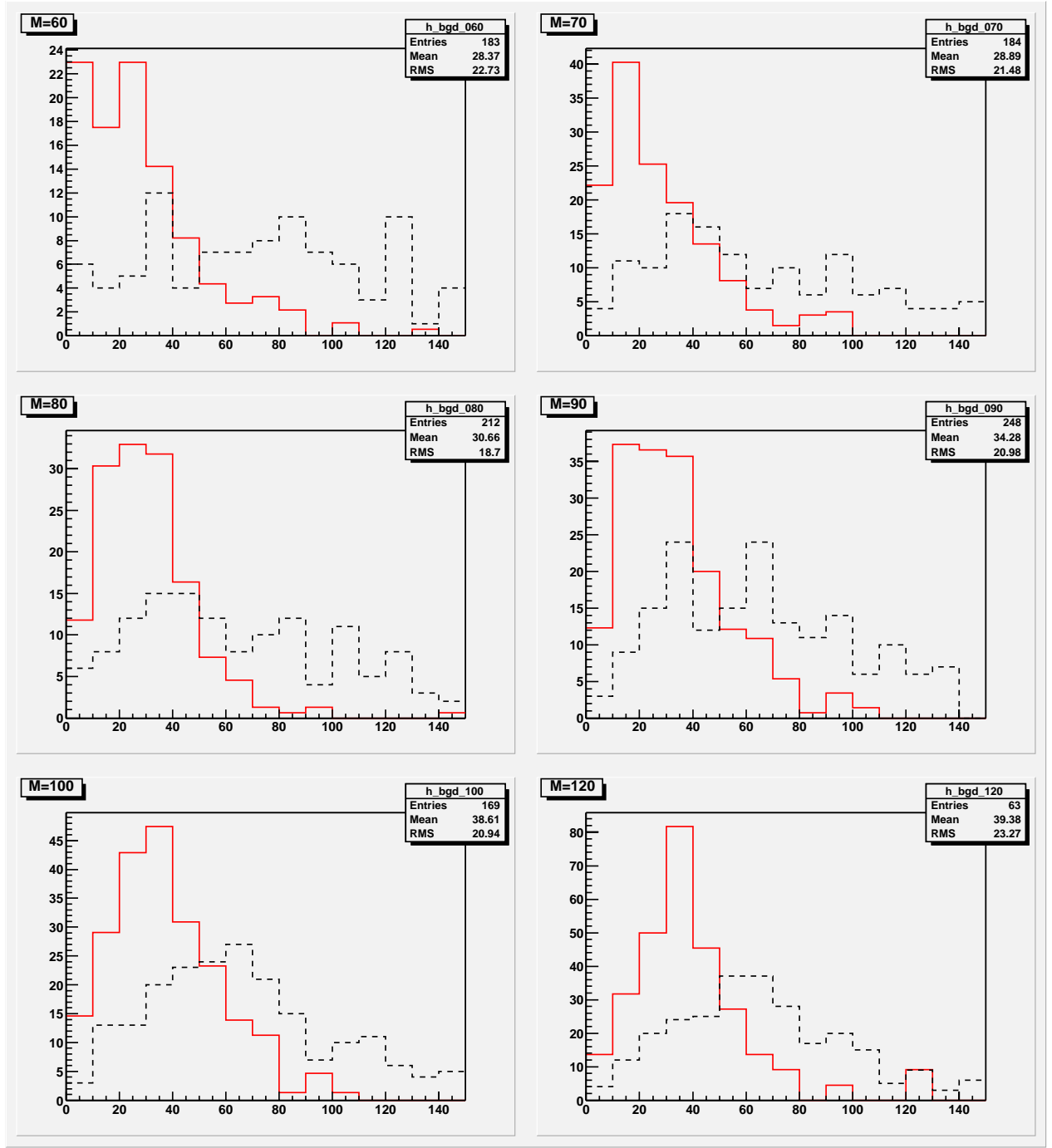

Figure 5.28: $p_{T}^{\gamma \gamma}$ distribution for signal (dashed line) and background (solid line) for the case when at least one jet with $p_{T}>30 \mathrm{GeV}$ is required to be present in the event 
multiplicity cut (as expected from the correlation between the $p_{T}^{\gamma \gamma}$ and jet multiplicity).

For quantitative comparison of various cuts we use significance (95\% CL cross-section limit averaged via Poisson distributin over all possible numbers of observed events [93]) defined as:

$$
\left\langle\sigma^{95}\right\rangle=\sum_{n=0}^{n=\infty} \sigma^{95}(n, S \mid B) e^{-B} \frac{B^{n}}{n !}
$$

where $n$ is the number of observed events, $S$ and $B$ are number of signal and background events, respectively. We refer to $\left\langle\sigma^{95}\right\rangle$ as the expected cross section limit.

We perform a scan of the $p_{T}^{\gamma \gamma}$ range and compute $\left\langle\sigma^{95}\right\rangle$ for each point in the scan [94] for the case of the jet multiplicity cut switched on and off. The results are shown in Fig. 5.29.

The conclusions from these studies are:

1. $p_{T}^{\gamma \gamma}$ cut provides significant improvement in the expected cross-section limit;

2. Addition of jets in the analysis does not improve the sensitivity.

We further extend these studies to the CCEC subset and include Topcolor Higgs using Higgs Monte Carlo samples of larger statistics.

Figures 5.30, 5.31, 5.32, and 5.33 show $p_{T}^{\gamma \gamma}$ distributions the signal and background for Fermiophobic Higgs (CCCC), Fermiophobic Higgs (CCEC), Topcolor Higgs (CCCC), and Topcolor Higgs (CCEC), respectively.

Figures 5.34, 5.35, 5.36, and 5.37 show Significance vs. $p_{T}^{\gamma \gamma}$ for Fermiophobic Higgs (CCCC), Fermiophobic Higgs (CCEC), Topcolor Higgs (CCCC), and Topcolor Higgs (CCEC), respectively. 

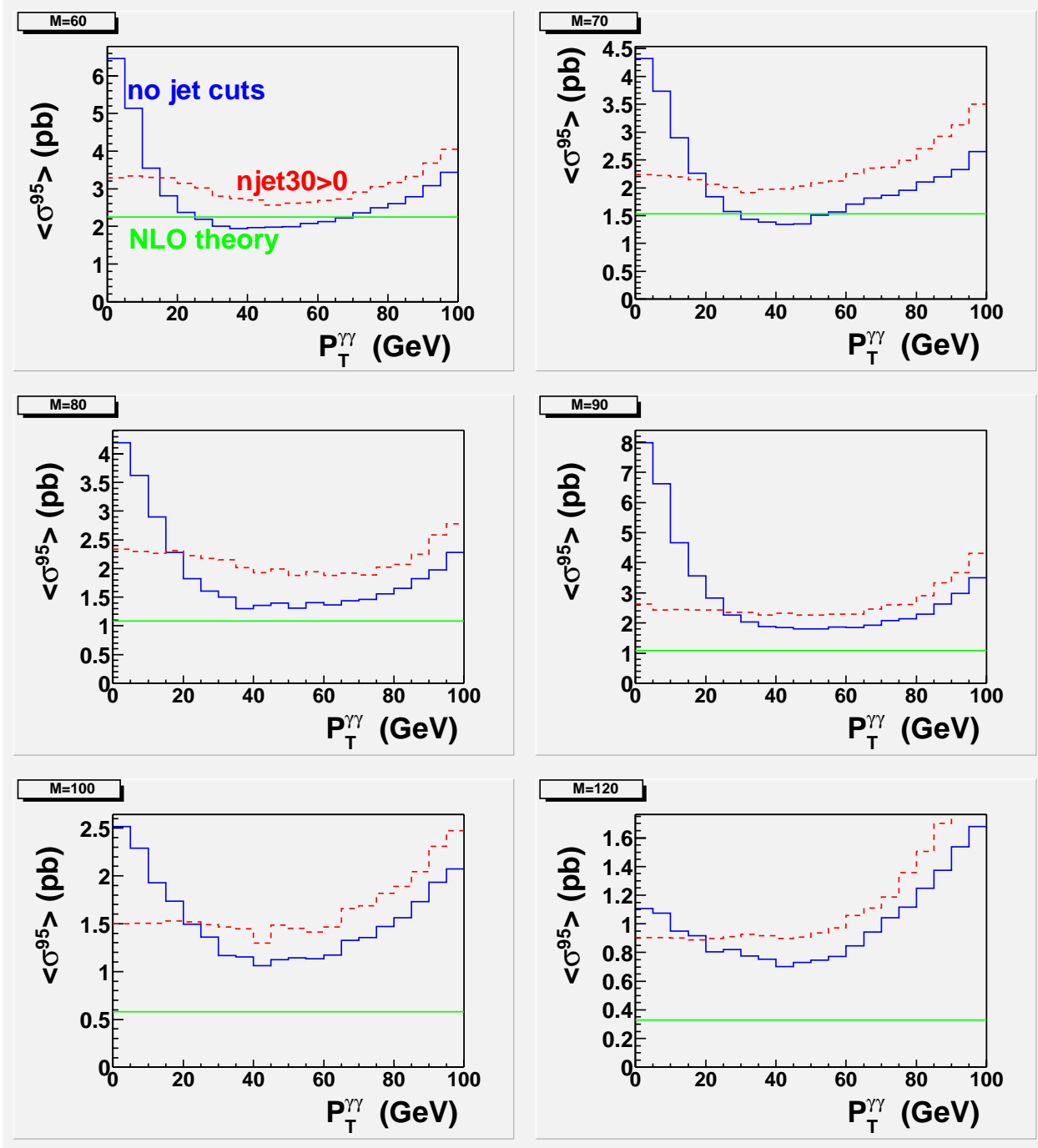

Figure 5.29: Significance as a function of the $p_{T}^{\gamma \gamma}$ cut. Solid curve: no jets are used in the analysis; red curve : at least one jet with $p_{T}>30 \mathrm{GeV}$ is required to be present in the event. Straight line shows the theoretical NLO Fermiophobic Higgs cross-section value (Assuming $B(\gamma \gamma)=1$ ). 

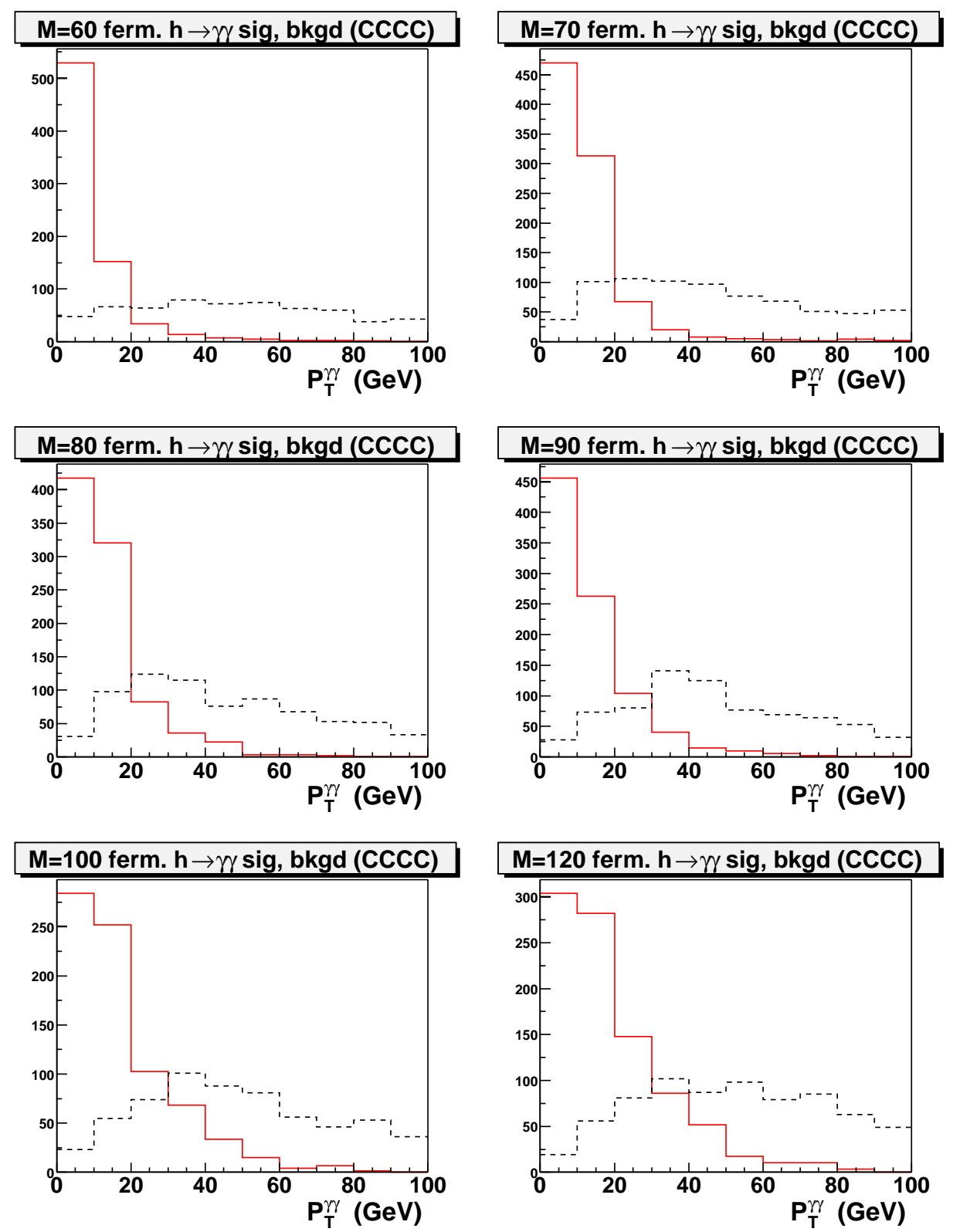

Figure 5.30: Fermiophobic Higgs CCCC $p_{T}^{\gamma \gamma}$ distributions. Dashed line - signal, solid line - background. 

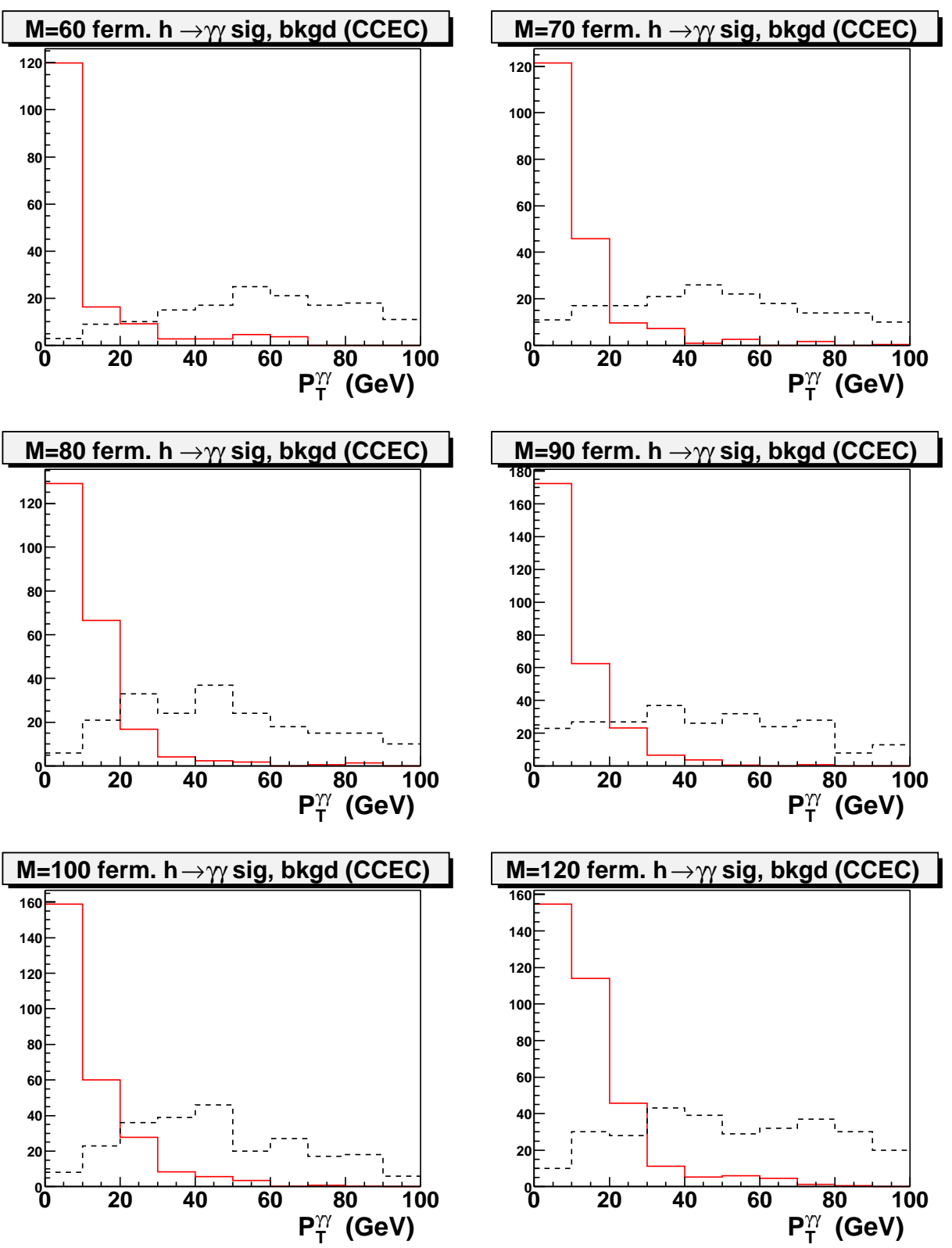

Figure 5.31: Fermiophobic Higgs CCEC $p_{T}^{\gamma \gamma}$ distributions. Dashed line - signal, solid line - background. 

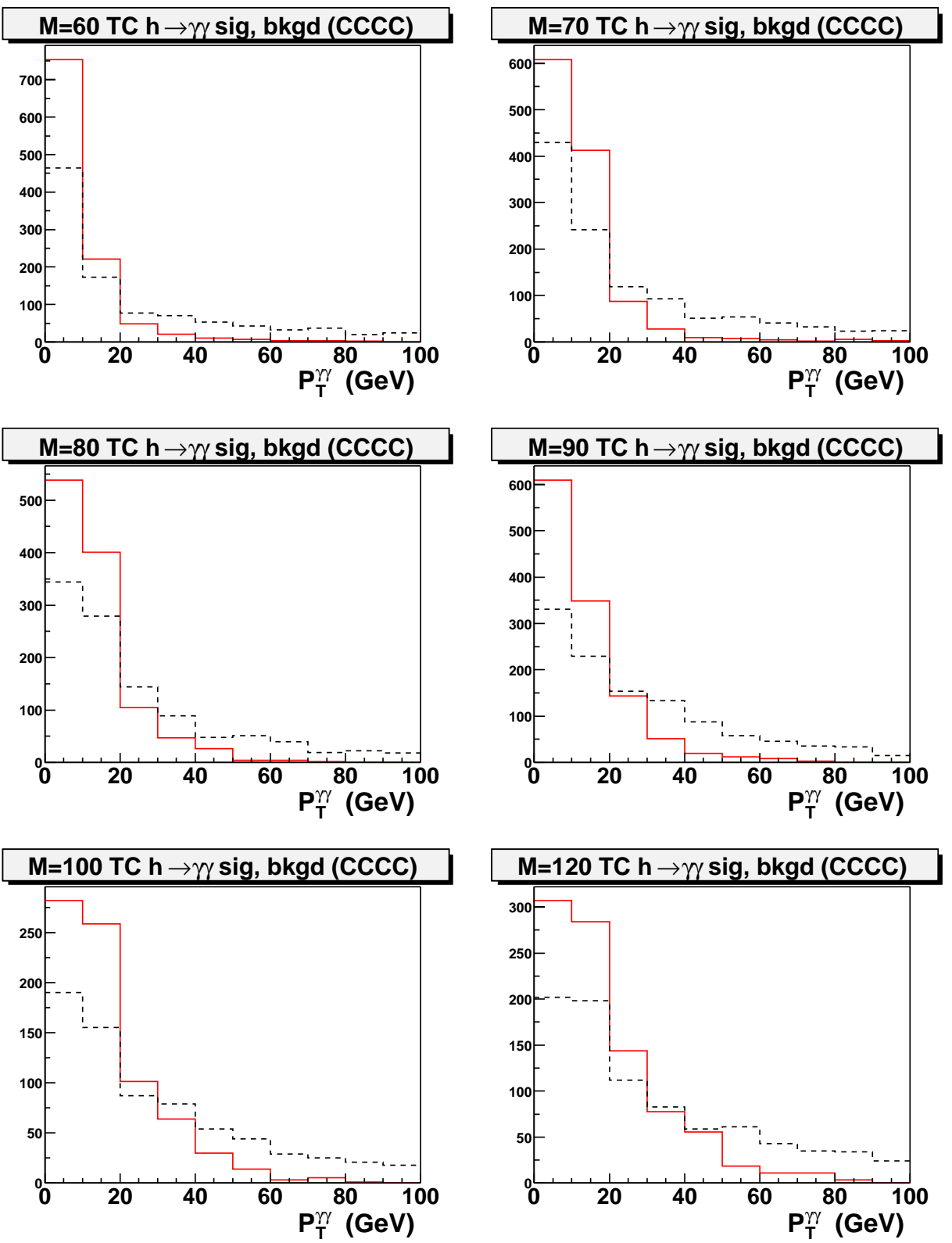

Figure 5.32: Topcolor Higgs CCCC $p_{T}^{\gamma \gamma}$ distributions. Dashed line - signal, solid line - background. 

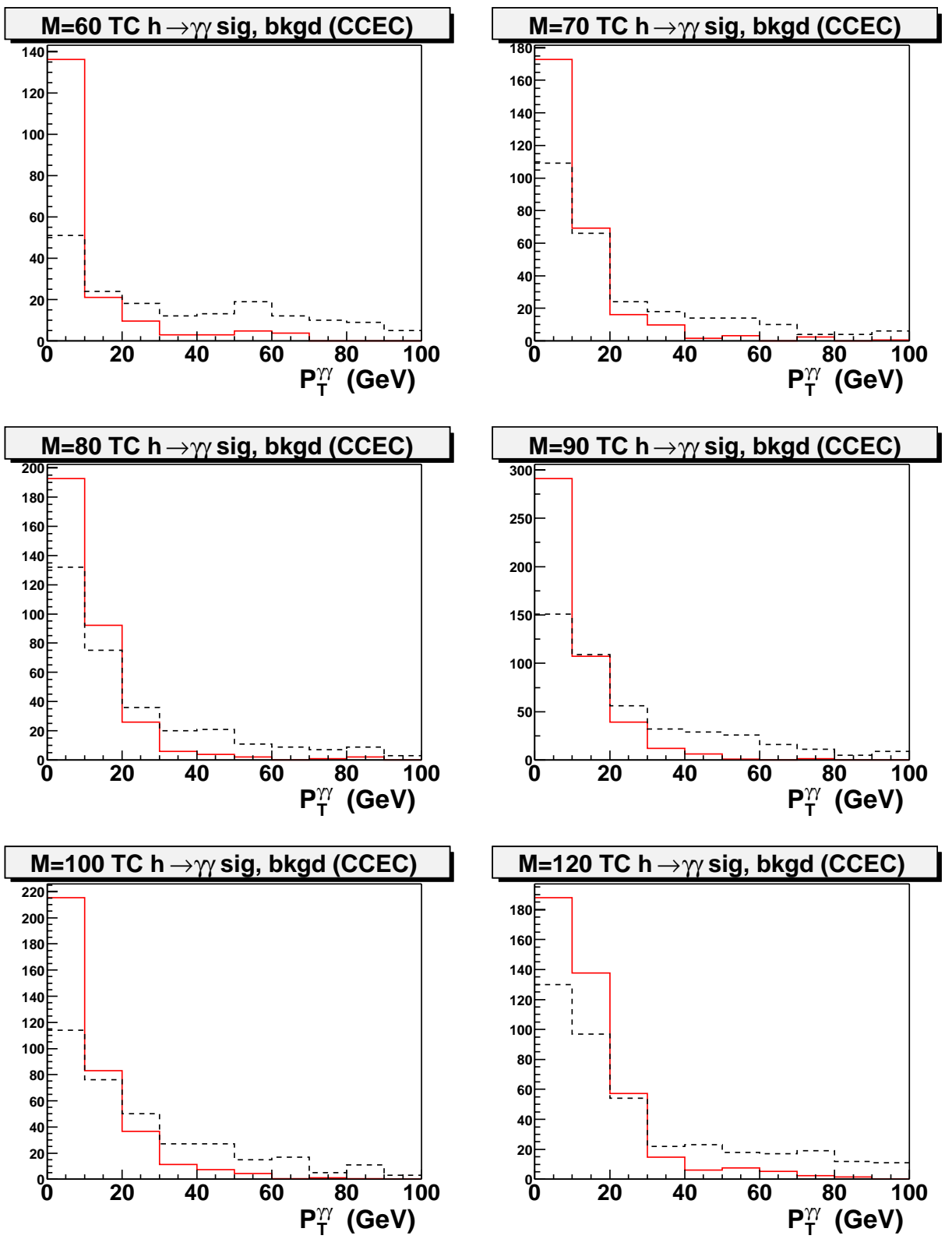

Figure 5.33: Topcolor Higgs CCEC $p_{T}^{\gamma \gamma}$ distributions. Dashed line - signal, solid line - background. 

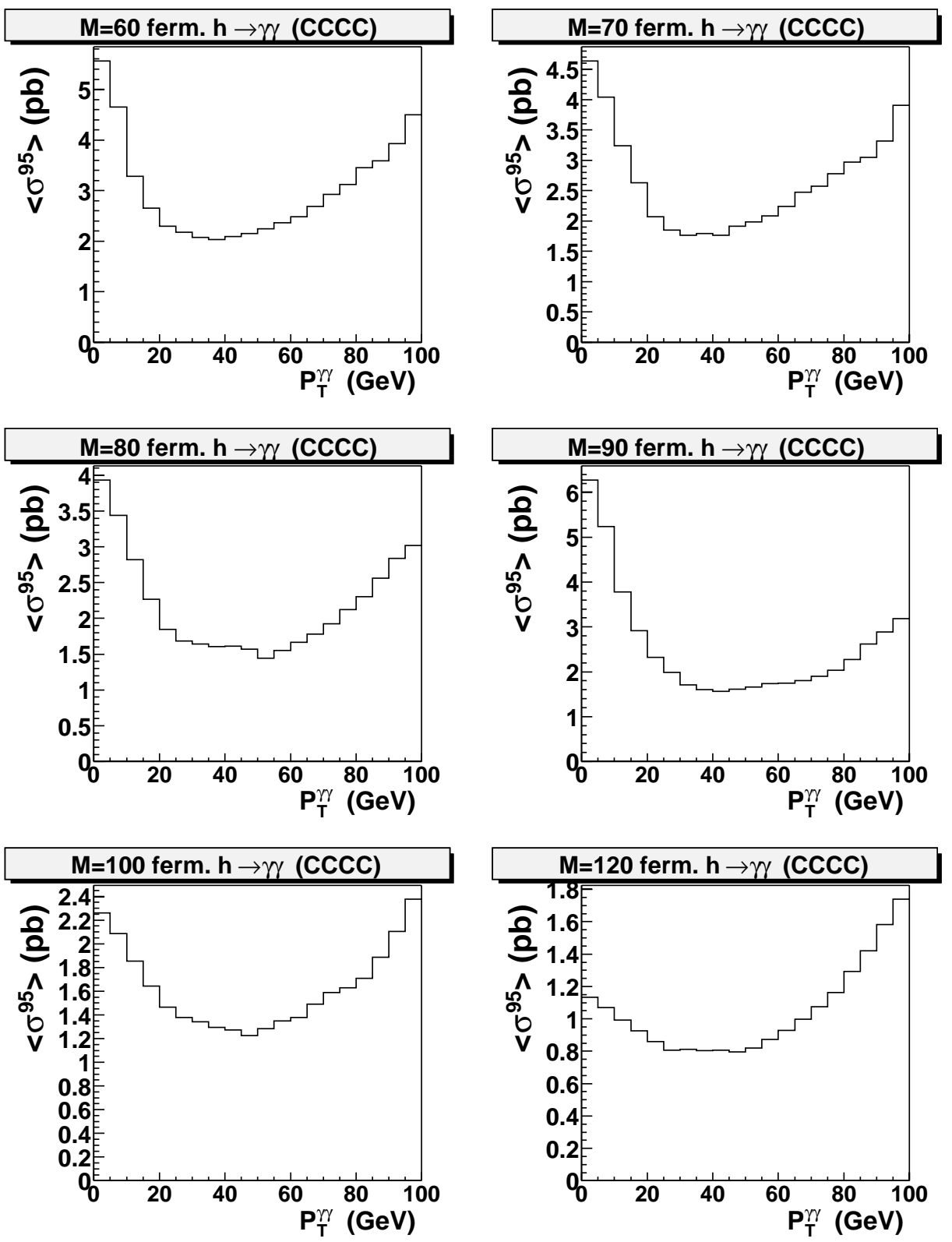

Figure 5.34: Fermiophobic Higgs CCCC Significance vs. $p_{T}^{\gamma \gamma}$. 

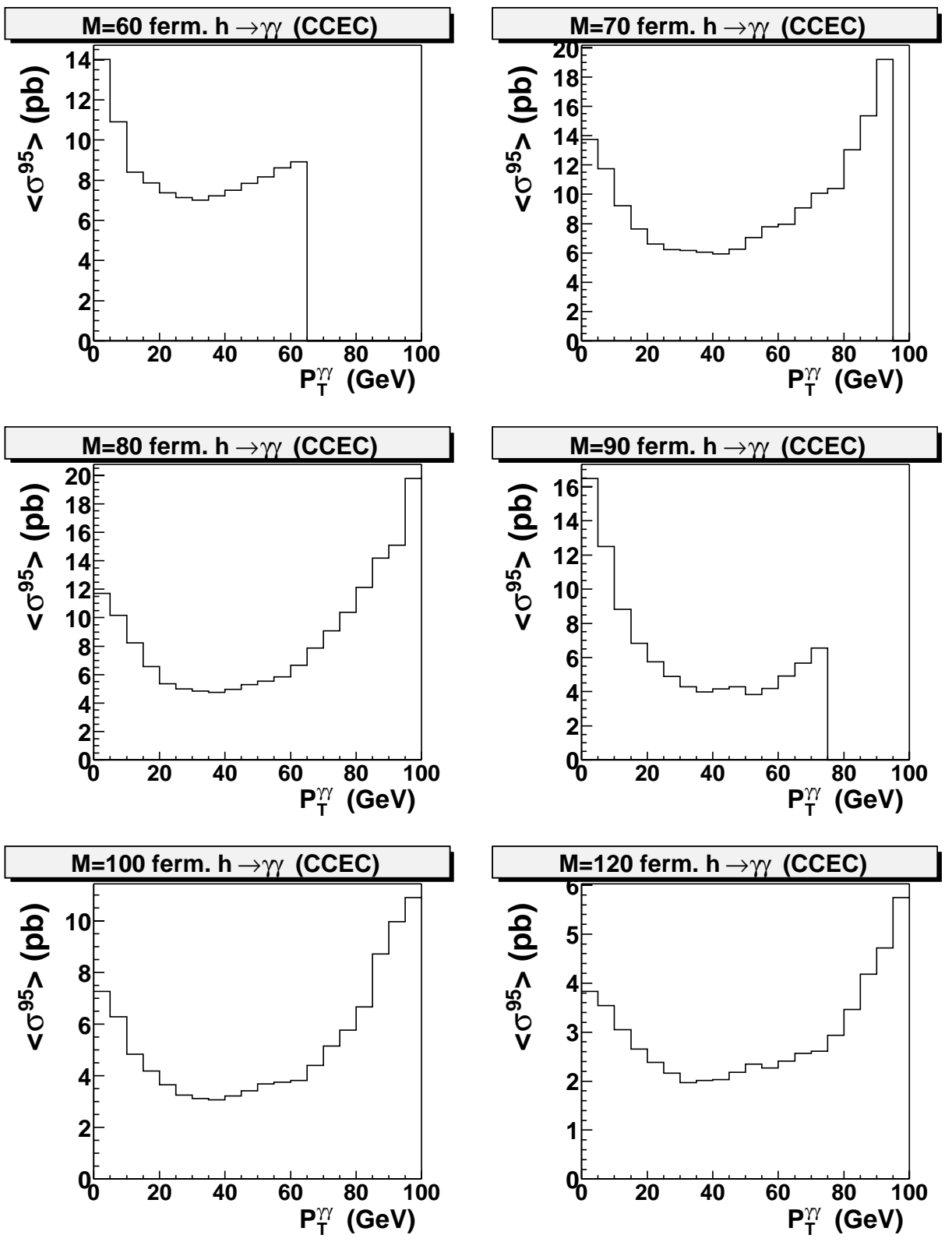

Figure 5.35: Fermiophobic Higgs CCEC Significance vs. $p_{T}^{\gamma \gamma}$. 

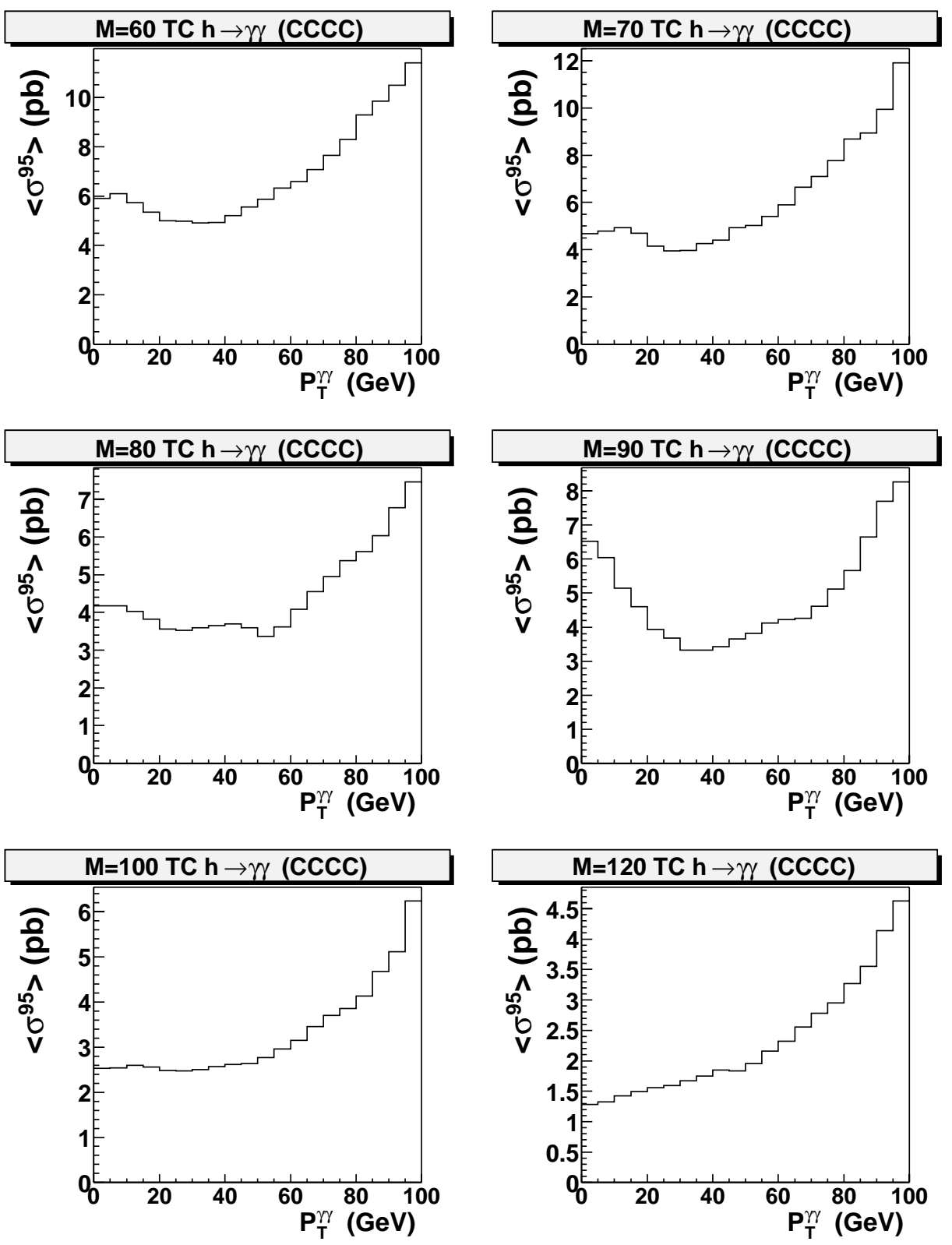

Figure 5.36: Topcolor Higgs CCCC Significance vs. $p_{T}^{\gamma \gamma}$. 

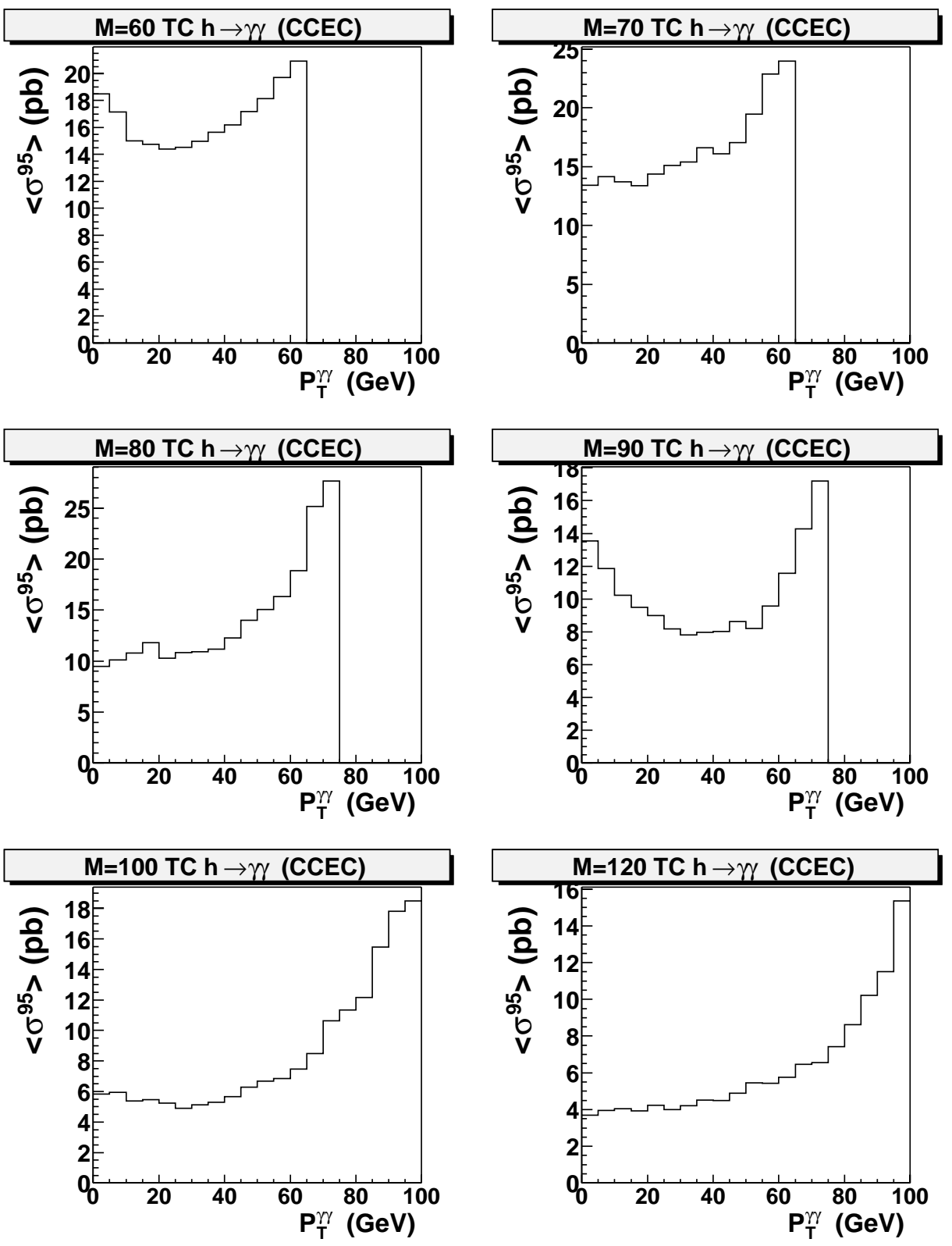

Figure 5.37: Topcolor Higgs CCEC Significance vs. $p_{T}^{\gamma \gamma}$. 
Based on these studies, we decide to use the following cuts:

- Fermiophobic Higgs: $p_{T}^{\gamma \gamma}>35.0 \mathrm{GeV}$ (for both CCCC and CCEC subsamples and all mass points);

- Topcolor Higgs: $p_{T}^{\gamma \gamma}>30.0 \mathrm{GeV}$ cut (for both CCCC and CCEC subsamples and all mass points, except $\mathrm{M}=120 \mathrm{GeV}$, where no $p_{T}^{\gamma \gamma}$ cut is applied).

\subsubsection{Event Counts After $p_{T}^{\gamma \gamma}$ cut}

Event counts after the optimal $p_{T}^{\gamma \gamma}$ cuts are applied are shown in Table 5.8.

\begin{tabular}{|c|c|c|c|c|}
\hline$M_{\mathrm{h}}, \mathrm{GeV}$ & Data & Total Background & QCD & Drell-Yan \\
\hline \multicolumn{5}{|c|}{ Fermiophobic Higgs CCCC } \\
\hline 60 & 4 & $2.9 \pm 0.7$ & $2.9 \pm 0.7$ & $0.0 \pm 0.0$ \\
\hline 70 & 5 & $1.1 \pm 1.1$ & $0.6 \pm 1.1$ & $0.6 \pm 0.3$ \\
\hline 80 & 3 & $3.2 \pm 1.4$ & $2.5 \pm 1.4$ & $0.7 \pm 0.4$ \\
\hline 90 & 7 & $7.3 \pm 2.7$ & $4.6 \pm 2.6$ & $2.7 \pm 0.9$ \\
\hline 100 & 4 & $2.5 \pm 1.3$ & $1.9 \pm 1.2$ & $0.6 \pm 0.3$ \\
\hline 120 & 1 & $1.7 \pm 0.6$ & $1.7 \pm 0.6$ & $0.0 \pm 0.0$ \\
\hline \multicolumn{5}{|c|}{ Fermiphobic Higgs CCEC } \\
\hline 60 & 4 & $1.0 \pm 0.5$ & $1.0 \pm 0.5$ & $0.0 \pm 0.0$ \\
\hline 70 & 1 & $0.8 \pm 1.2$ & $0.1 \pm 1.1$ & $0.7 \pm 0.5$ \\
\hline 80 & 4 & $2.2 \pm 1.0$ & $1.8 \pm 1.0$ & $0.4 \pm 0.4$ \\
\hline 90 & 5 & $6.2 \pm 2.1$ & $4.5 \pm 2.0$ & $1.8 \pm 0.8$ \\
\hline 100 & 2 & $2.9 \pm 1.8$ & $1.4 \pm 1.6$ & $1.4 \pm 0.7$ \\
\hline 120 & 4 & $2.7 \pm 0.8$ & $2.7 \pm 0.8$ & $0.0 \pm 0.0$ \\
\hline \multicolumn{5}{|c|}{ Topcolor Higgs CCCC } \\
\hline 60 & 6 & $4.4 \pm 1.0$ & $4.4 \pm 1.0$ & $0.0 \pm 0.0$ \\
\hline 70 & 7 & $2.5 \pm 1.3$ & $1.9 \pm 1.2$ & $0.6 \pm 0.3$ \\
\hline 80 & 5 & $6.9 \pm 1.7$ & $6.2 \pm 1.7$ & $0.7 \pm 0.4$ \\
\hline 90 & 9 & $10.2 \pm 3.2$ & $6.7 \pm 3.0$ & $3.5 \pm 1.1$ \\
\hline 100 & 8 & $4.9 \pm 1.6$ & $4.0 \pm 1.6$ & $0.8 \pm 0.4$ \\
\hline 120 & 7 & $9.2 \pm 1.8$ & $8.7 \pm 1.7$ & $0.4 \pm 0.3$ \\
\hline \multicolumn{5}{|c|}{ Topcolor Higgs CCEC } \\
\hline 60 & 6 & $2.1 \pm 0.7$ & $2.1 \pm 0.7$ & $0.0 \pm 0.0$ \\
\hline 70 & 1 & $2.5 \pm 1.3$ & $1.7 \pm 1.2$ & $0.7 \pm 0.5$ \\
\hline 80 & 5 & $3.6 \pm 1.2$ & $3.2 \pm 1.1$ & $0.4 \pm 0.4$ \\
\hline 90 & 8 & $8.6 \pm 2.9$ & $5.0 \pm 2.7$ & $3.6 \pm 1.2$ \\
\hline 100 & 3 & $5.0 \pm 2.1$ & $3.2 \pm 1.9$ & $1.8 \pm 0.8$ \\
\hline 120 & 34 & $31.2 \pm 3.6$ & $29.0 \pm 3.5$ & $2.1 \pm 0.9$ \\
\hline
\end{tabular}

Table 5.8: CCCC event count, expected signal and backgrounds

Generally the agreement between the data and predicted background is good. However, there 
are several points were discrepancy is quite large. For example, for $\mathrm{M}=70 \mathrm{GeV}$ Fermiophobic Higgs (CCCC subsample) we predict $1.1 \pm 1.1$ events from the SM background and observe 5 events. The probability for such background fluctuation (including error) is less than $8 \%$. Another point is CCEC M=60 Fermiophobic Higgs with $1.0 \pm 0.5$ events predicted and 4 observed. The discrepancies in these two points are replicated in the case of Topcolor Higgs as well since the difference in events counts comes only from small variations in the size of the mass window.

We rule out possible interpretation of this discrepancy by additional unaccounted for backgrounds such as $W \gamma$ and $W+$ jets processes on the basis of the following arguments :

- Our DPF 2002 Conference $W \gamma$ MC studies [95] suggest that in the current sample one event should be expected given current tracking efficiency and $p_{T}>20 \mathrm{GeV}$ cut on each photon. Currently $p_{T}>25 \mathrm{GeV}$ cut is used. Therefore the probability to have $W \gamma$ in the sample is very low. Similar argument applies to the $W+$ jets case given current tracking efficiency and Photon misidentification rate.

- Moreover di-Photon invariant mass spectrum in $W \gamma$ and $W+$ jets is expected to peak below 60-70 GeV.

- All of the observed events contain at least one jet. From the cross-section considerations follows that it is more probable to observe first $W \gamma / W+$ jets events without an extra jet.

- Finally, Missing $E_{T}$ in in the observed events is often found to be aligned with a jet. Therefore it is likely to come from jet energy mismeasurement rather than from a neutrino in the $\mathrm{W}$ decay (top plots in Figs. 5.38 and 5.39).

We interpret the discrepancy as coming from the down-fluctuation of the QCD component of the background [96]. Looking at the wide mass range we notice that QCD background shape has a dip in the regions where the above discrepancies are observed (Figs. 5.40 and 5.41).

Such behaviour of the QCD background is not consistent with the QCD kinematics and must come from the fluctuation. As a cross-check, we relax the mass window cut in successive steps and make sure that the significance of the observed excess decreases with increased mass window size. This supports our explanation of the discrepancy by the down-fluctuation of the background. The 

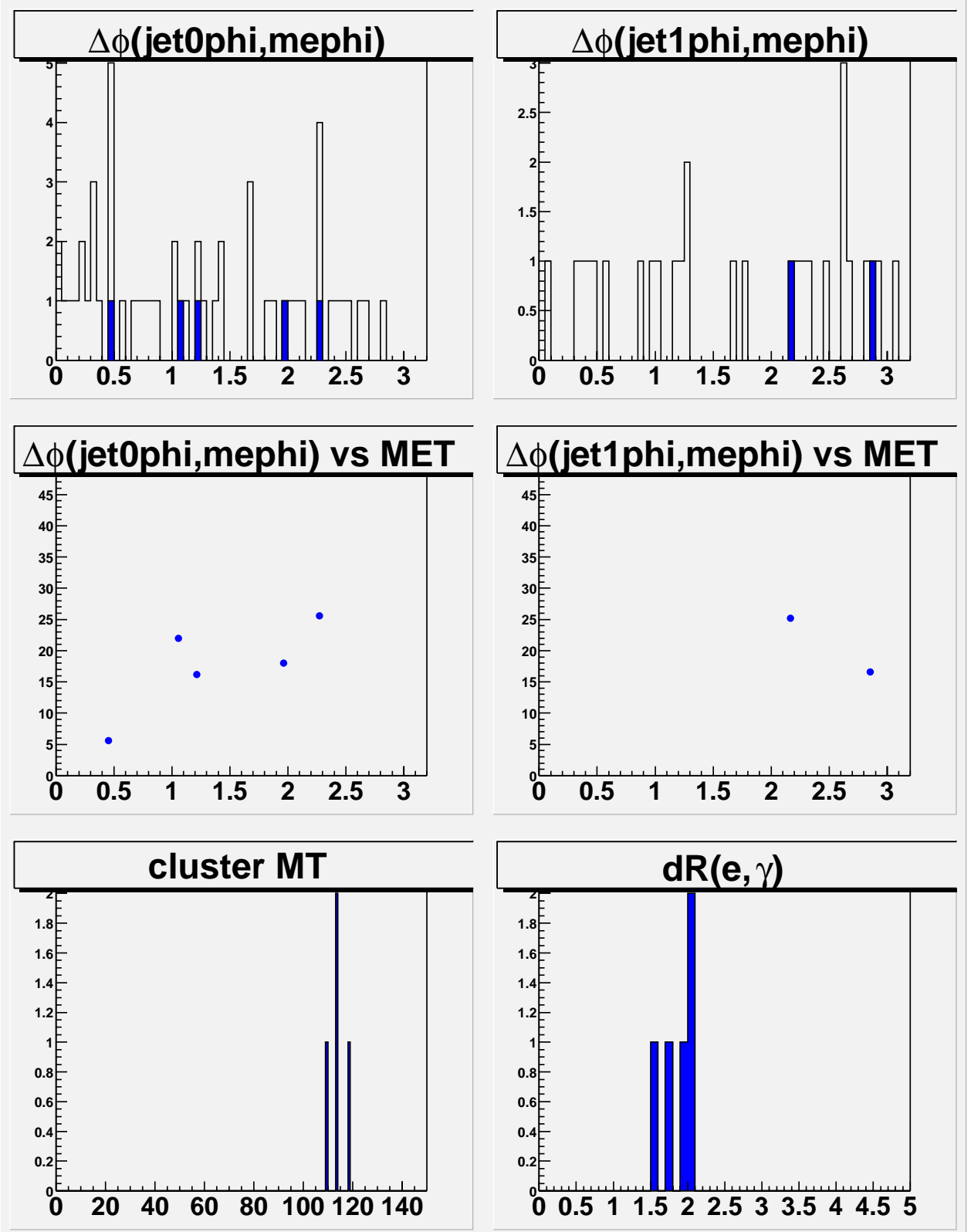

Figure 5.38: Studying background/data discrepancy for $\mathrm{M}=70 \mathrm{GeV}$ CCCC Fermiophobic Higgs. Top plots show $\Delta \phi$ between the Missing Et and a Jet: top left plot corresponds to the leading jet, top right plot corresponds to the trailing jet. Filled histogram shows observed events after $p_{T}^{\gamma \gamma}$ cut; open histogram corresponds to the case when $p_{T}^{\gamma \gamma}$ cut is removed. Middle plots show $\Delta \phi$ of the top plots vs. Missing Et. Bottom left plot shows Cluster Transverse Mass distribution; bottom right plot shows $d R(\eta, \phi)$ separation between two photons (a photon and potential $W \rightarrow e \nu$ electron). 

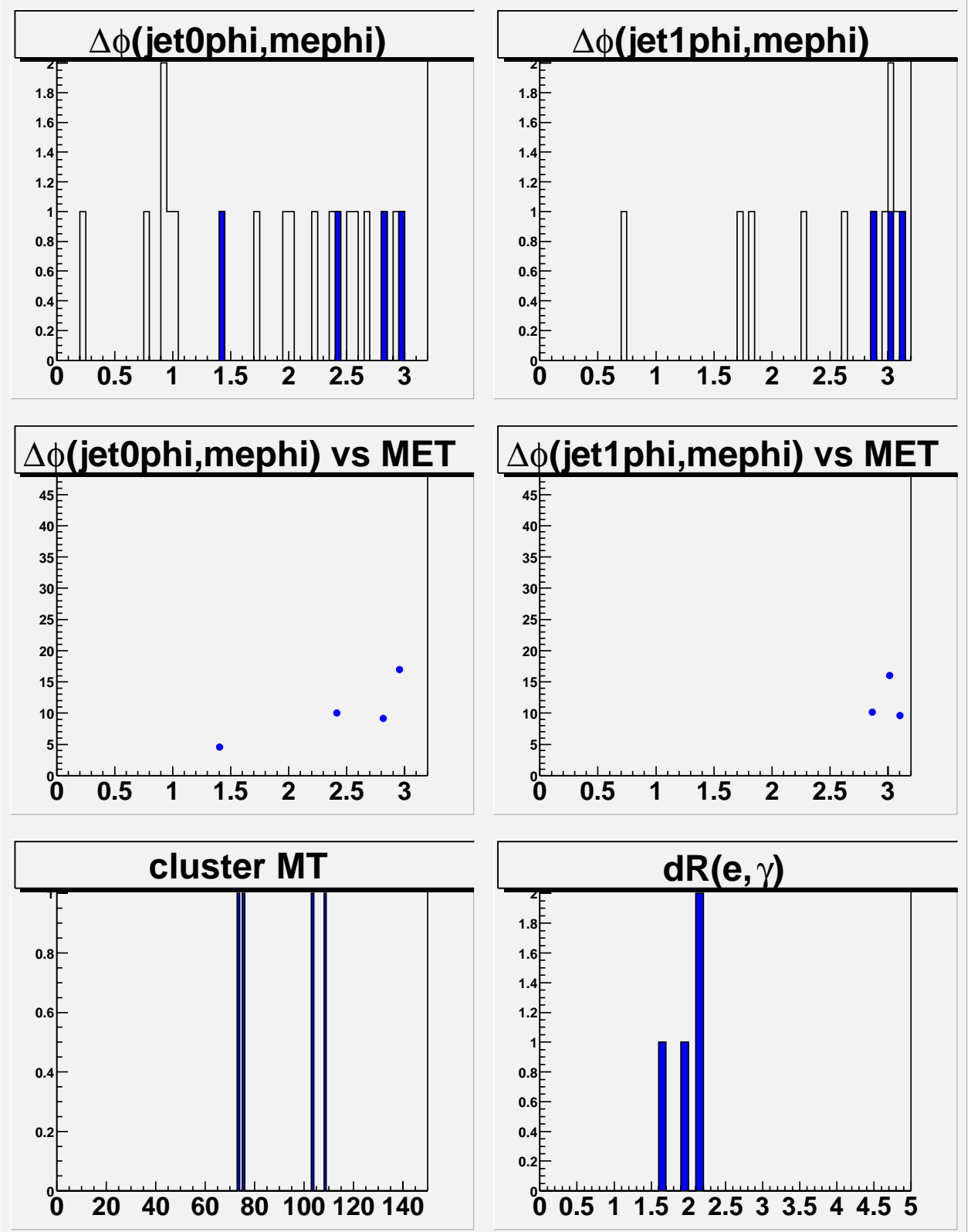

Figure 5.39: Studying background/data disrcepancy for $\mathrm{M}=60 \mathrm{GeV}$ CCEC Fermiophobic Higgs. Top plots show $\Delta \phi$ between the Missing Et and a Jet: top left plot corresponds to the leading jet, top right plot corresponds to the trailing jet. Filled histogram shows observed events after $p_{T}^{\gamma \gamma}$ cut; open histogram corresponds to the case when $p_{T}^{\gamma \gamma}$ cut is removed. Middle plots show $\Delta \phi$ of the top plots vs. Missing Et. Bottom left plot shows Cluster Transverse Mass distribution; bottom right plot shows $d R(\eta, \phi)$ separation between two photons (a photon and potential $W \rightarrow e \nu$ electron). 


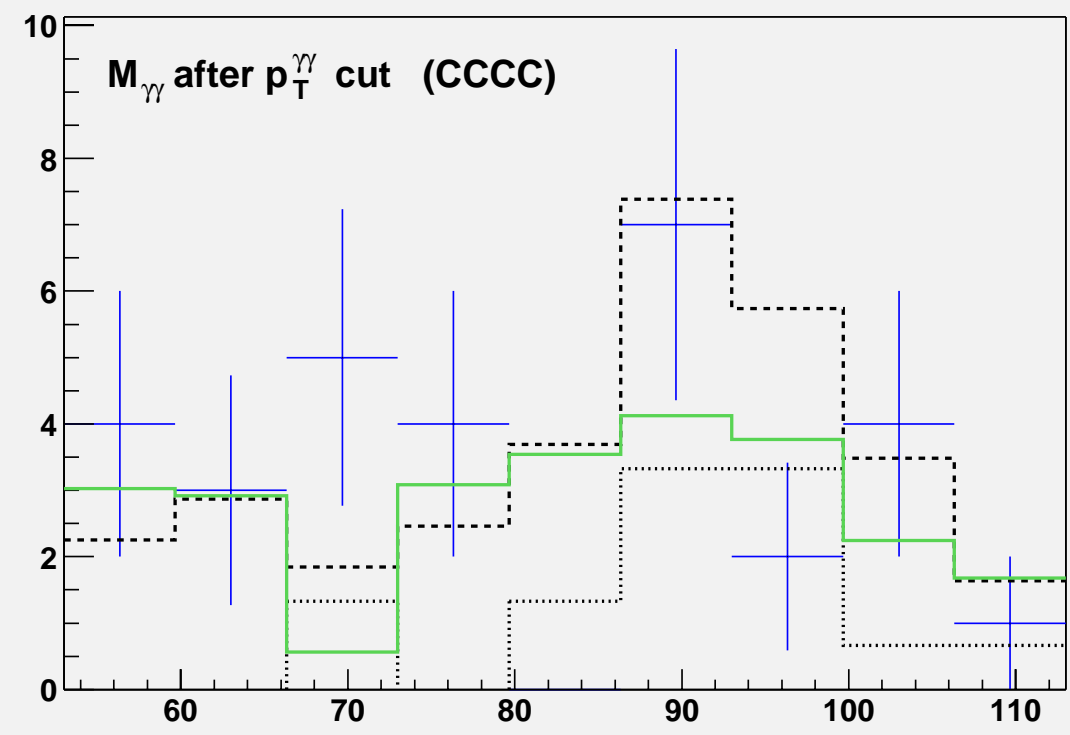

Figure 5.40: CCCC $\gamma \gamma$ invariant mass distribution for the QCD background after $p_{T}^{\gamma \gamma}$ cut is applied. Dashed and dotted line histograms show two of the QCD background terms as described by equation 5.8. solid shows total QCD background, points show data.

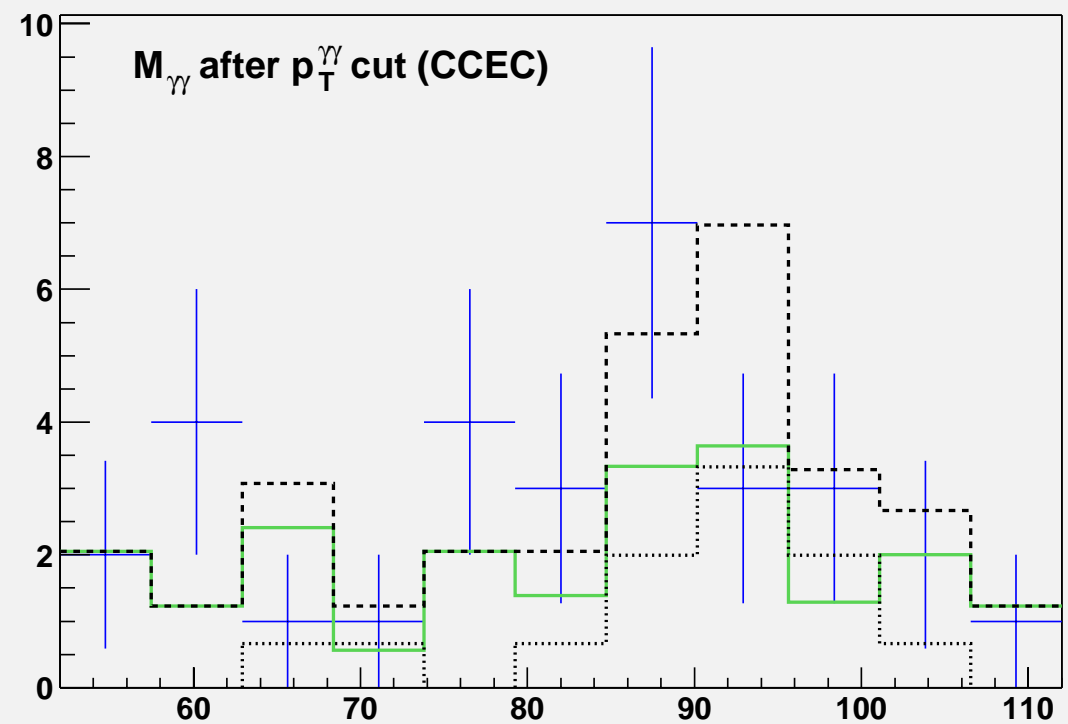

Figure 5.41: CCEC $\gamma \gamma$ invariant mass distribution for the QCD background after $p_{T}^{\gamma \gamma}$ cut is applied. Dashed and dotted line histograms show two of the QCD background terms as described by equation 5.8. solid line shows total QCD background, points show data. 
results of such verification are shown in Tables 5.9 and 5.10 for $\mathrm{CCCC} M=70 \mathrm{GeV}$ and $\mathrm{CCEC} \mathrm{M}=60$ $\mathrm{GeV}$ points respectively.

\begin{tabular}{cccc}
\hline$M_{\mathrm{h}}, \mathrm{GeV}$ & Window $/ 2, \mathrm{GeV}$ & Background & Data \\
\hline 70 & 3.21 & $1.1 \pm 1.1$ & 5 \\
70 & 3.50 & $1.1 \pm 1.1$ & 5 \\
70 & 3.75 & $1.3 \pm 1.2$ & 5 \\
70 & 4.00 & $1.5 \pm 1.2$ & 5 \\
70 & 4.25 & $2.0 \pm 1.2$ & 5 \\
70 & 4.50 & $2.0 \pm 1.3$ & 5 \\
70 & 5.00 & $2.2 \pm 1.3$ & 5 \\
70 & 5.50 & $2.5 \pm 1.4$ & 5 \\
70 & 6.00 & $2.7 \pm 1.4$ & 5 \\
70 & 7.00 & $4.5 \pm 1.5$ & 7 \\
70 & 8.00 & $4.9 \pm 1.5$ & 9 \\
\hline
\end{tabular}

Table 5.9: Predicted background and observed number of events as a function of the mass window size $(\mathrm{CCCC}, \mathrm{M}=70 \mathrm{GeV})$.

\begin{tabular}{cccc}
\hline$M_{\mathrm{h}}, \mathrm{GeV}$ & Window $/ 2, \mathrm{GeV}$ & Background & Data \\
\hline 60 & 2.68 & $1.0 \pm 0.5$ & 4 \\
60 & 3.00 & $1.6 \pm 0.6$ & 5 \\
60 & 3.25 & $1.6 \pm 0.6$ & 5 \\
60 & 3.50 & $1.8 \pm 0.6$ & 5 \\
60 & 3.75 & $2.1 \pm 0.7$ & 5 \\
60 & 4.00 & $2.3 \pm 0.7$ & 5 \\
60 & 4.25 & $2.7 \pm 0.8$ & 5 \\
60 & 4.50 & $3.1 \pm 0.8$ & 5 \\
60 & 5.00 & $3.7 \pm 0.9$ & 5 \\
60 & 5.50 & $4.3 \pm 1.0$ & 5 \\
60 & 6.00 & $4.7 \pm 1.0$ & 5 \\
60 & 7.00 & $5.3 \pm 1.1$ & 6 \\
60 & 8.00 & $5.9 \pm 1.2$ & 7 \\
\hline
\end{tabular}

Table 5.10: Predicted background and number of observed events as a function of a mass window size (CCEC, $\mathrm{M}=60 \mathrm{GeV}$ ). 


\subsubsection{Other Kinematic Variables : $d R^{\gamma \gamma}(\eta, \phi),(E 1-E 2) /(E 1+E 2),\left|\cos \theta^{*}\right|$}

Having established the optimal $p_{T}^{\gamma \gamma}$ cuts and verified that the backgrounds describe the data after these cuts are applied, we proceed with exploring other kinematic variables. These variables are $d R^{\gamma \gamma}(\eta, \phi),(E 1-E 2) /(E 1+E 2),\left|\cos \theta^{*}\right|$. We start with $d R^{\gamma \gamma}(\eta, \phi)$ and apply it on top of the $p_{T}^{\gamma \gamma}$ cut.

Figures $5.42,5.43,5.44$, and 5.45 show signal and background $d R^{\gamma \gamma}(\eta, \phi)$ distributions for Fermiophobic Higgs (CCCC), Fermiophobic Higgs (CCEC), Topcolor Higgs (CCCC), and Topcolor Higgs (CCEC), respectively. 

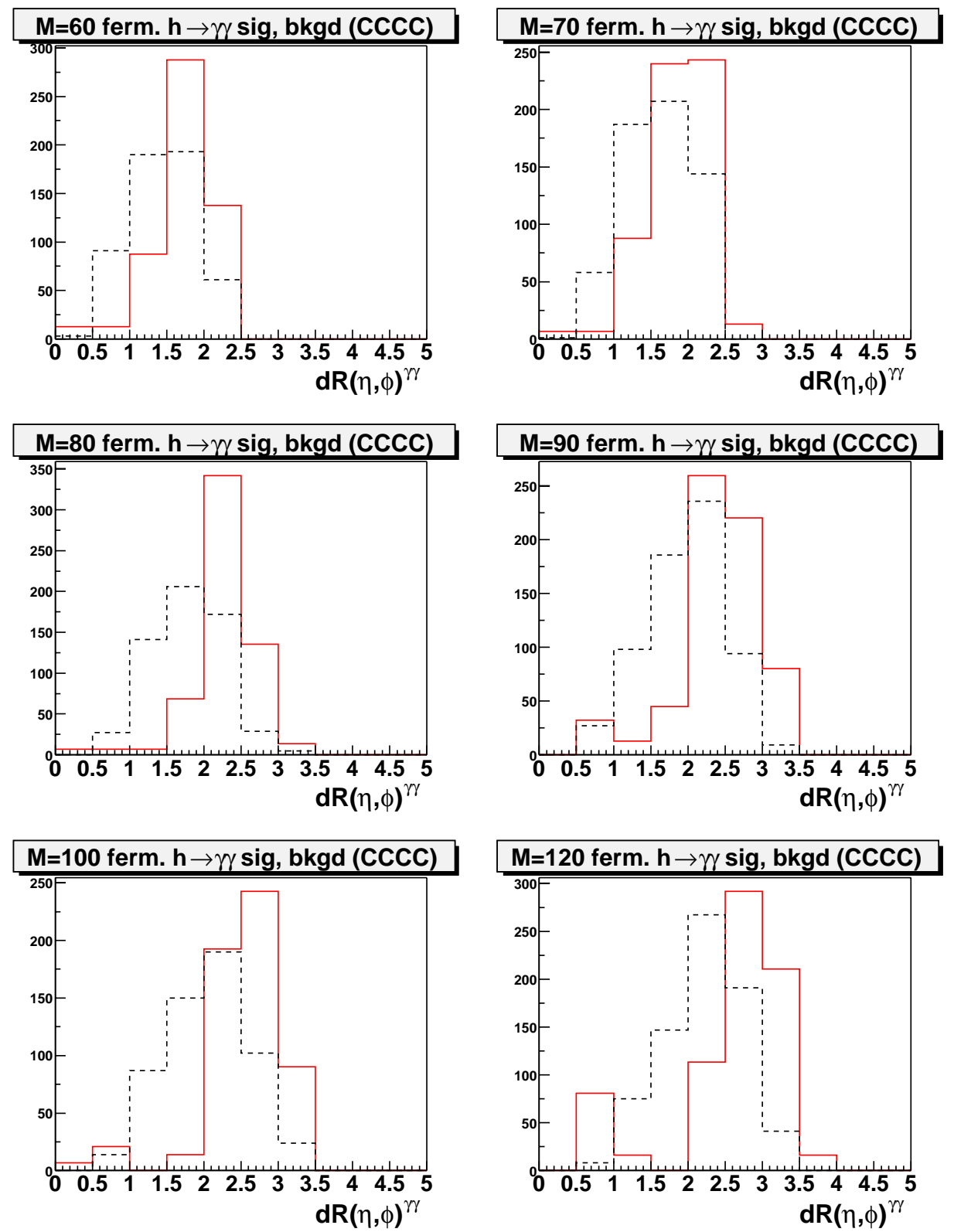

Figure 5.42: Fermiophobic Higgs CCCC $d R^{\gamma \gamma}(\eta, \phi)$ after the $p_{T}^{\gamma \gamma}$ cut. Dashed line - signal, solid line - background. 

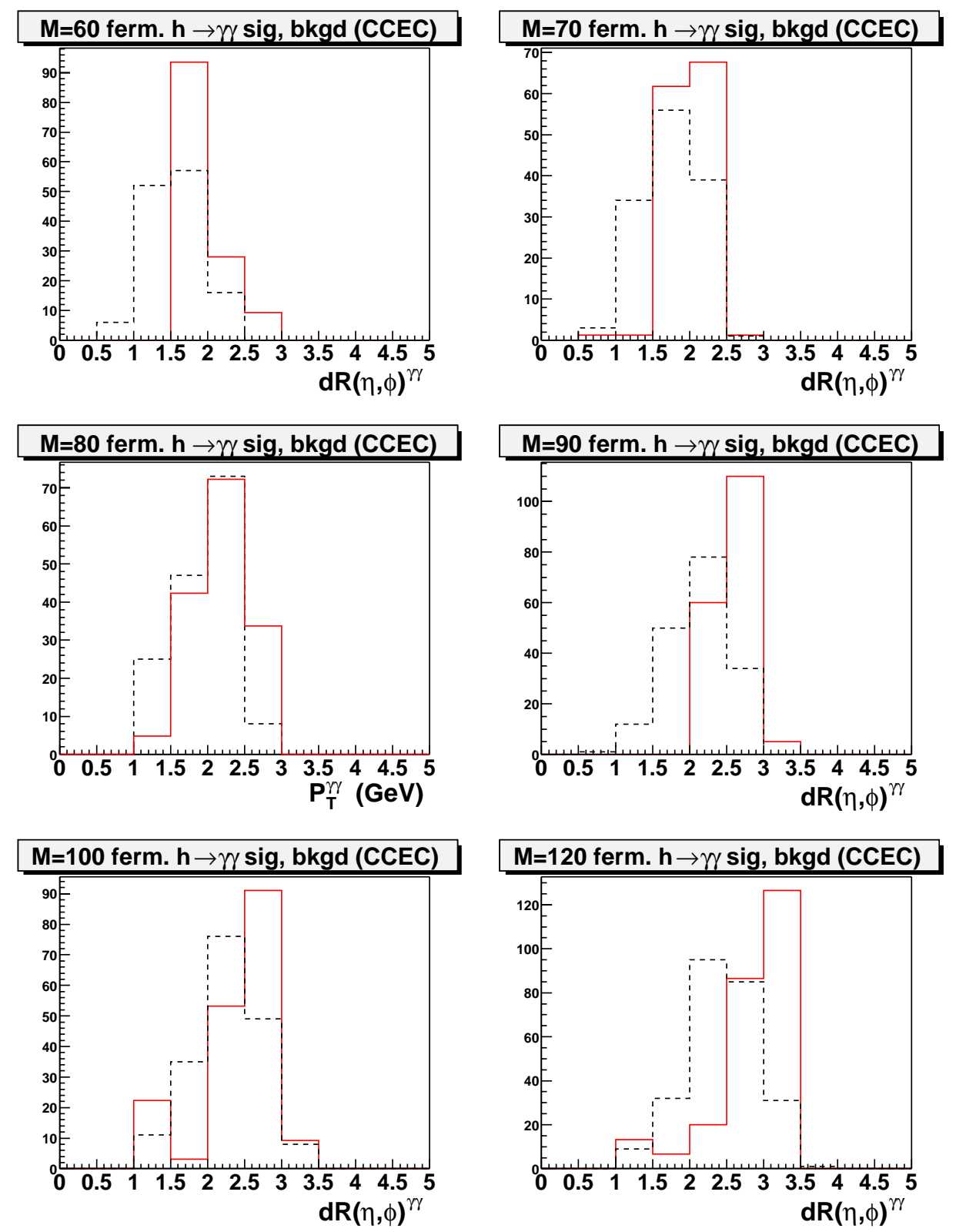

Figure 5.43: Fermiophobic Higgs CCEC $d R^{\gamma \gamma}(\eta, \phi)$ after the $p_{T}^{\gamma \gamma}$ cut. Dashed line - signal, solid line - background. 

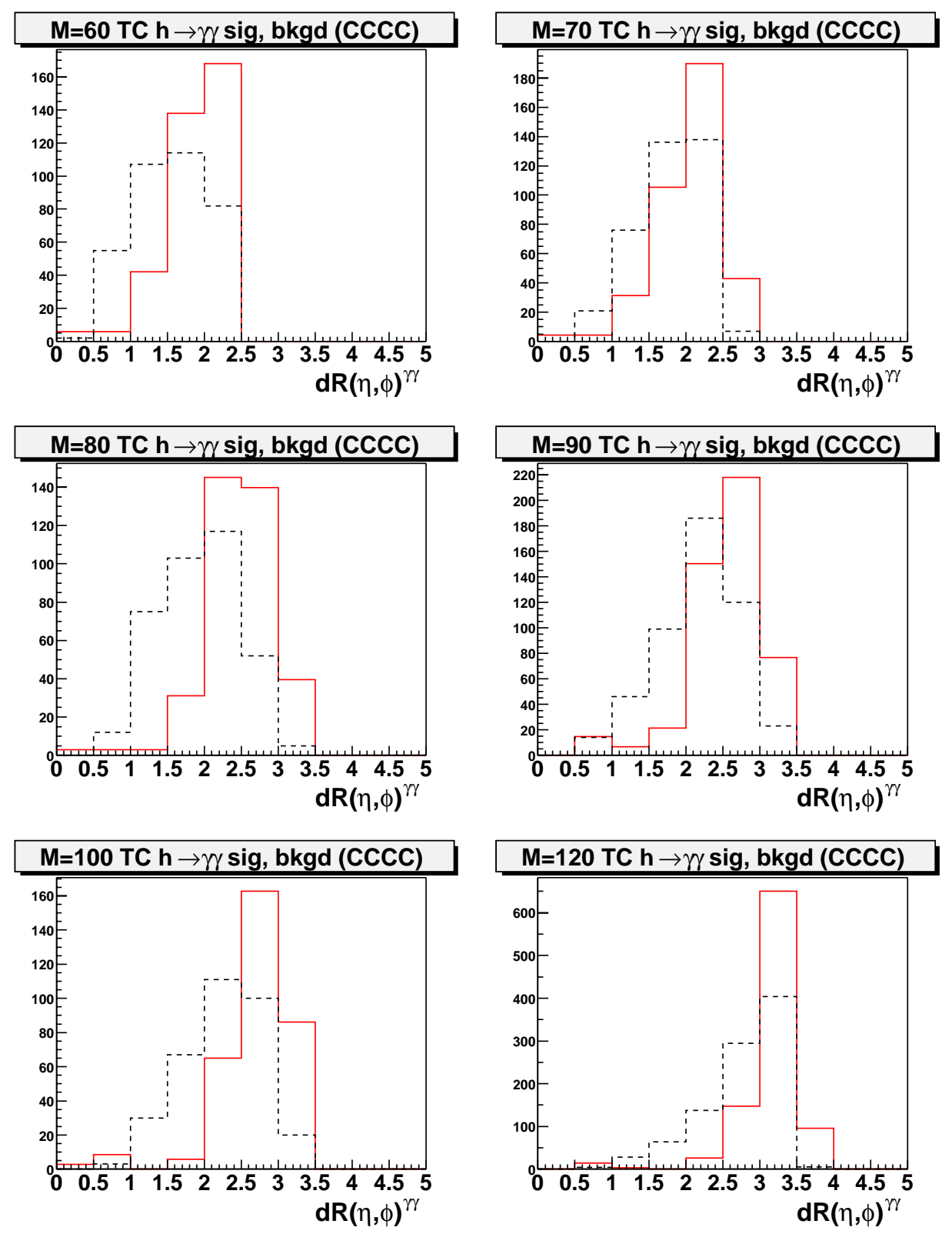

Figure 5.44: Topcolor Higgs CCCC $d R^{\gamma \gamma}(\eta, \phi)$ after the $p_{T}^{\gamma \gamma}$ cut. Dashed line - signal, solid line background. 

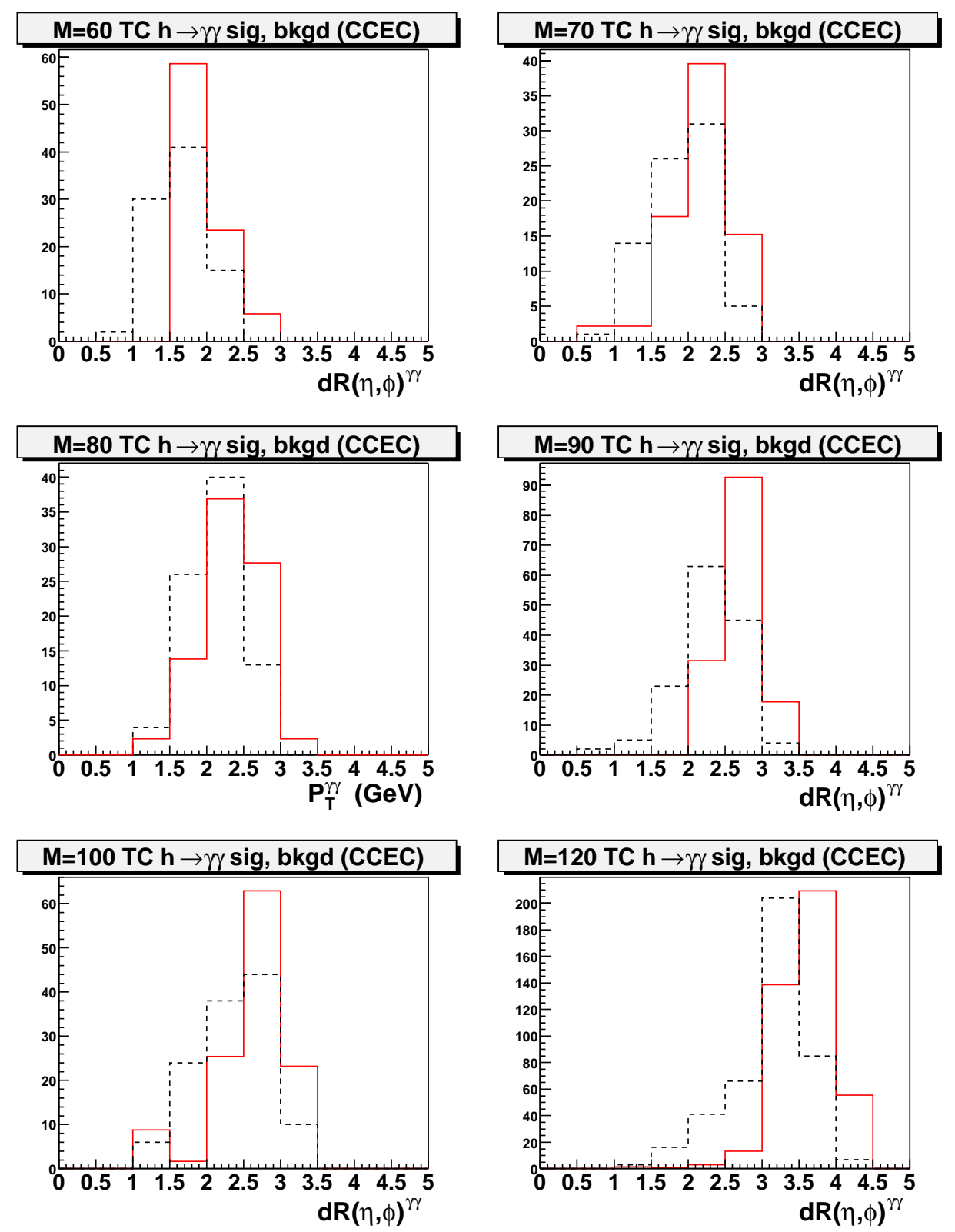

Figure 5.45: Topcolor Higgs CCEC $d R^{\gamma \gamma}(\eta, \phi)$ after the $p_{T}^{\gamma \gamma}$ cut. Dashed line - signal, solid line background.

The size of the background sample used for these plots is small, as $p_{T}^{\gamma \gamma}$ cut already eliminated significant fraction of the background.

Figures 5.46, 5.47, 5.48, and 5.49 show Significance vs. $d R^{\gamma \gamma}(\eta, \phi)$ for Fermiophobic Higgs (CCCC), Fermiophobic Higgs (CCEC), Topcolor Higgs (CCCC), and Topcolor Higgs (CCEC), respectively. 

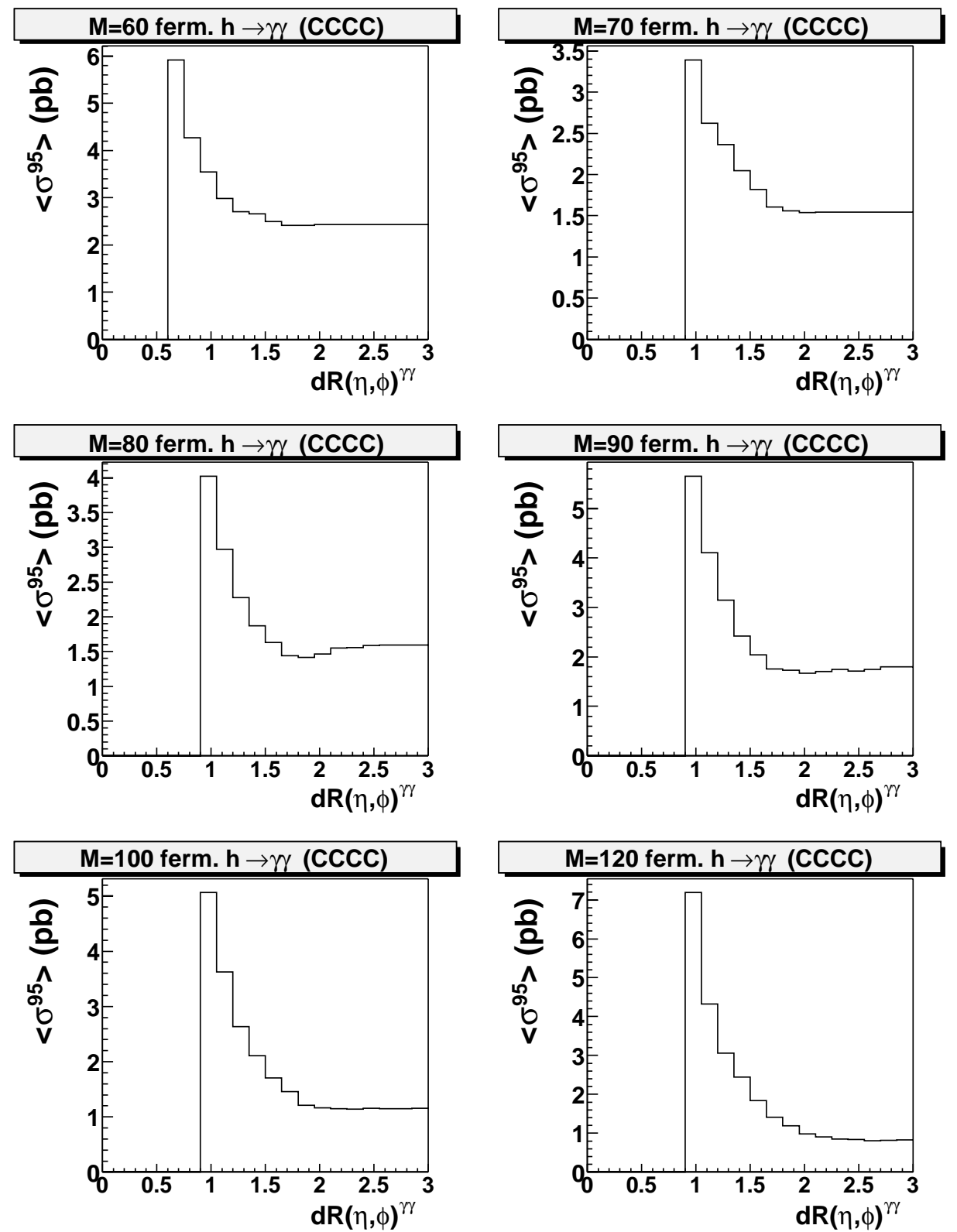

Figure 5.46: Fermiophobic Higgs CCCC Significance vs. $d R^{\gamma \gamma}(\eta, \phi)$ after the $p_{T}^{\gamma \gamma}$ cut is applied. Region of acceptance $=100 \%$ is on the right. 

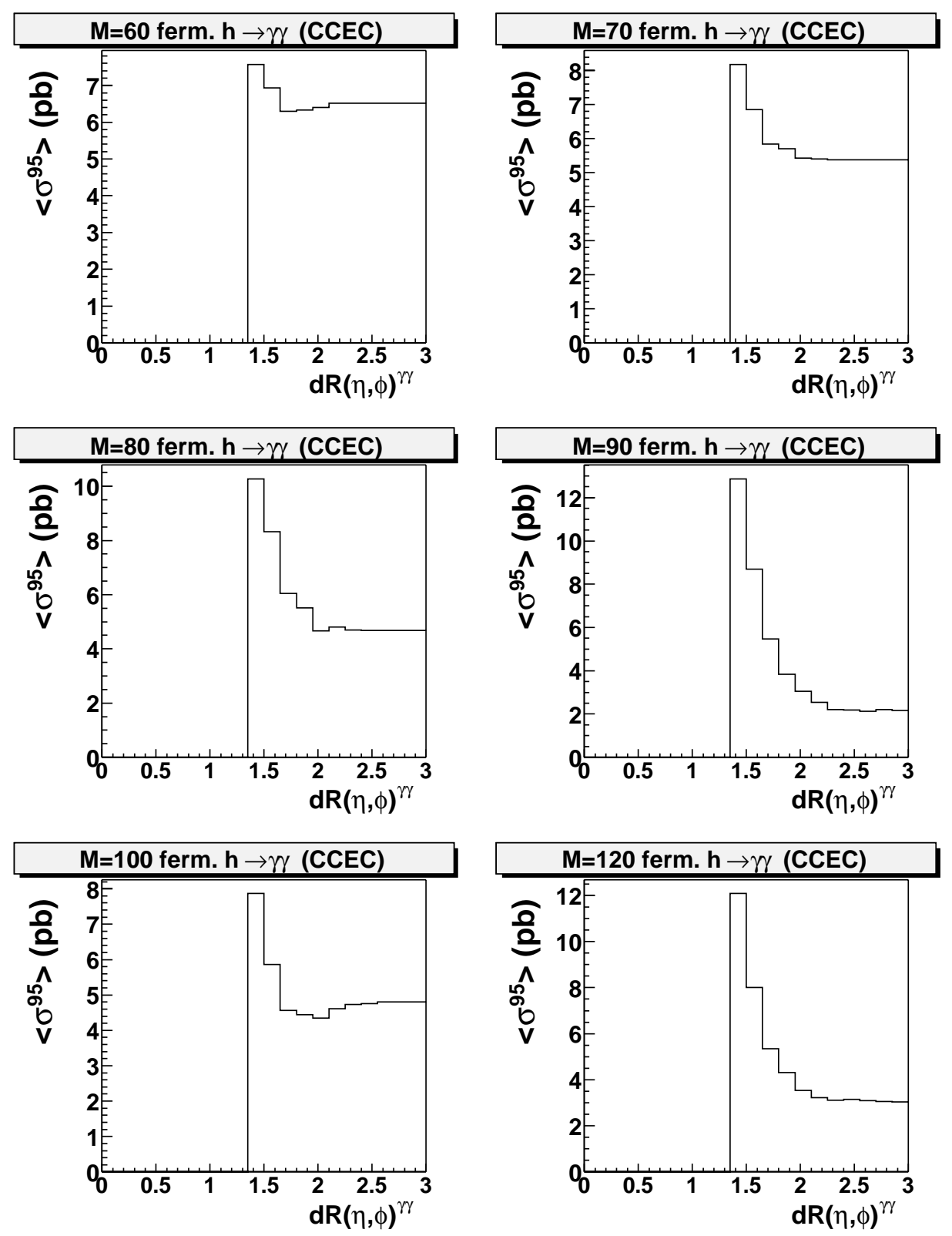

Figure 5.47: Fermiophobic Higgs CCEC Significance vs. $d R^{\gamma \gamma}(\eta, \phi)$ after the $p_{T}^{\gamma \gamma}$ cut is applied. Region of acceptance $=100 \%$ is on the right. 

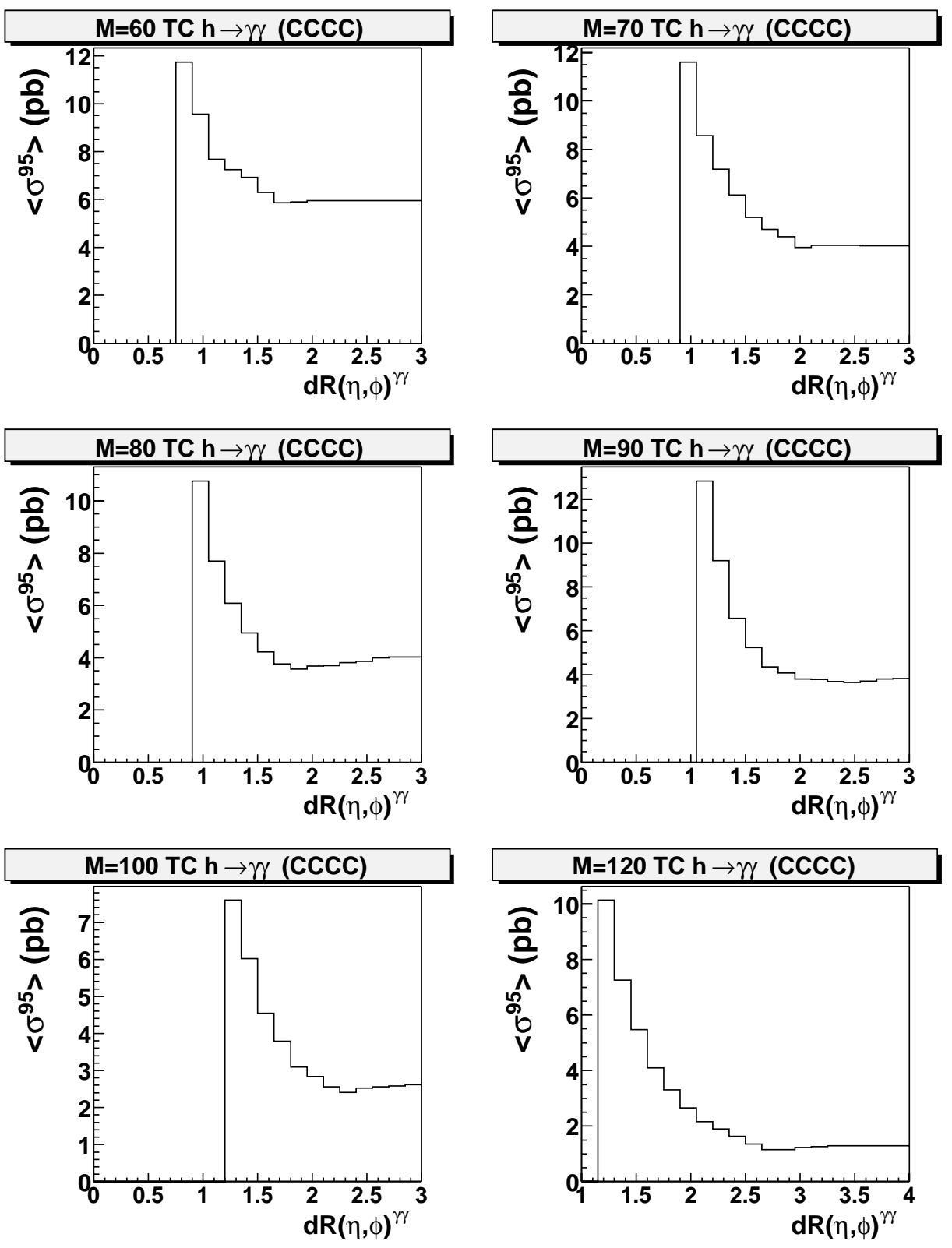

Figure 5.48: Topcolor Higgs CCCC Significance vs. $d R^{\gamma \gamma}(\eta, \phi)$ after the $p_{T}^{\gamma \gamma}$ cut is applied. Region of acceptance $=100 \%$ is on the right. 

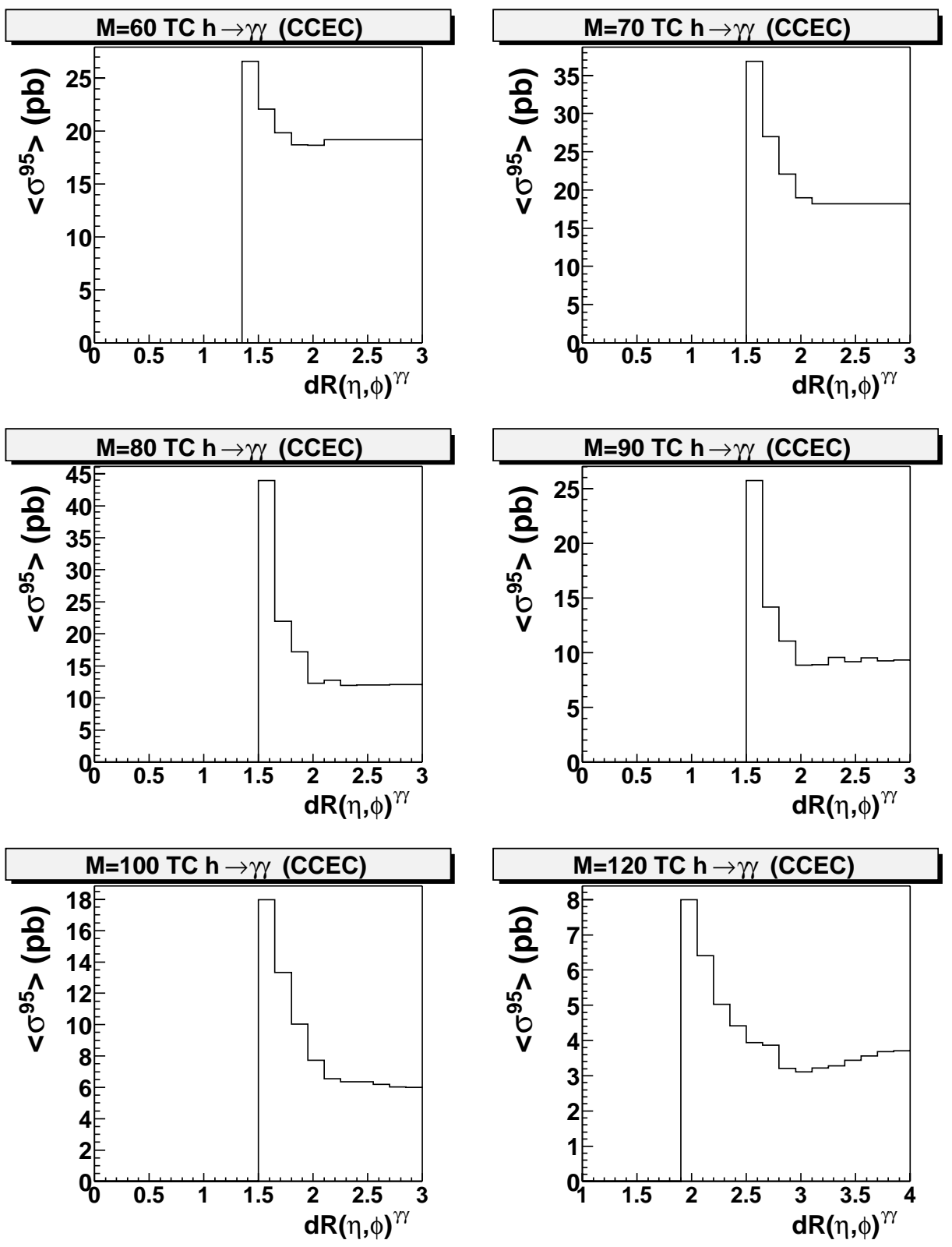

Figure 5.49: Topcolor Higgs CCEC Significance vs. $d R^{\gamma \gamma}(\eta, \phi)$ after the $p_{T}^{\gamma \gamma}$ cut is applied. Region of acceptance $=100 \%$ is on the right.

From the Significance vs. $d R^{\gamma \gamma}(\eta, \phi)$ plots we conclude that $d R^{\gamma \gamma}(\eta, \phi)$ does not improve the significance and therefore should not be used. This can be explained by the fact that $d R^{\gamma \gamma}(\eta, \phi)$ and $p_{T}^{\gamma \gamma}$ are expected to be correlated, as shown in the top plot of Figure 5.50.

Such correlation may be traced to the $p_{T}^{\gamma \gamma}$ correlation with $\Delta \phi$ between the two photons (the middle plot in Figure 5.50). Therefore we also look separately at the $\Delta \eta$ distributions for the signal 


\section{dr:diphpt $\{$ cccc $==1 \& \&$ pt $[0]>25 \& \&$ pt $[1]>25 \& \&$ diphpt $<100\}$}

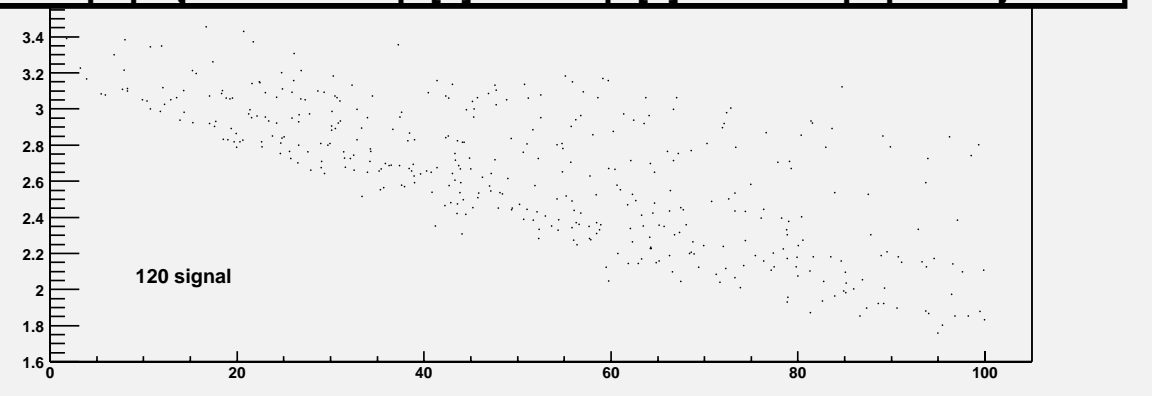

abs(deltadphi):diphpt $\{$ cccc==1 \&\& pt[0]>25 \&\& pt[1]>25 \&\& diphpt<100\}

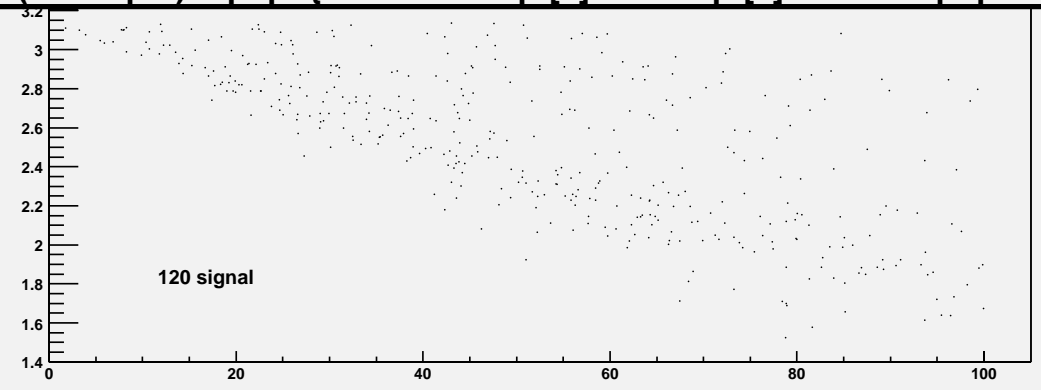

assymetry:diphpt $\{\mathrm{cccc}==1 \& \& \mathrm{pt}[0]>25 \& \& \mathrm{pt}[1]>25 \& \&$ diphpt $<100\}$

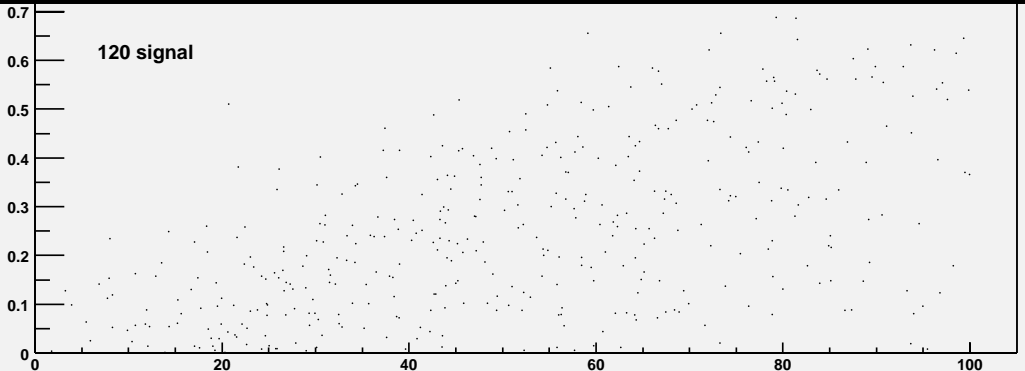

Figure 5.50: An example of correlations of $p_{T}^{\gamma \gamma}$ with other kinematic variables for $\mathrm{M}=120 \mathrm{GeV}$ Fermiophobic Higgs (CCCC) Top: $d R^{\gamma \gamma}(\eta, \phi)$ (vertical) vs. $p_{T}^{\gamma \gamma}$ (horizontal). Middle: $\Delta \phi$ (vertical) vs. $p_{T}^{\gamma \gamma}$ (horizontal). Bottom: $(E 1-E 2) /(E 1+E 2)$ (vertical) vs. $p_{T}^{\gamma \gamma}$ (horizontal). 
and background, which are shown in Figs. 5.51, 5.52, 5.53, and 5.54 for Fermiophobic Higgs (CCCC), Fermiophobic Higgs (CCEC), Topcolor Higgs (CCCC), and Topcolor Higgs (CCEC), respectively. No significant separation is achieved with $\Delta \eta$ either.
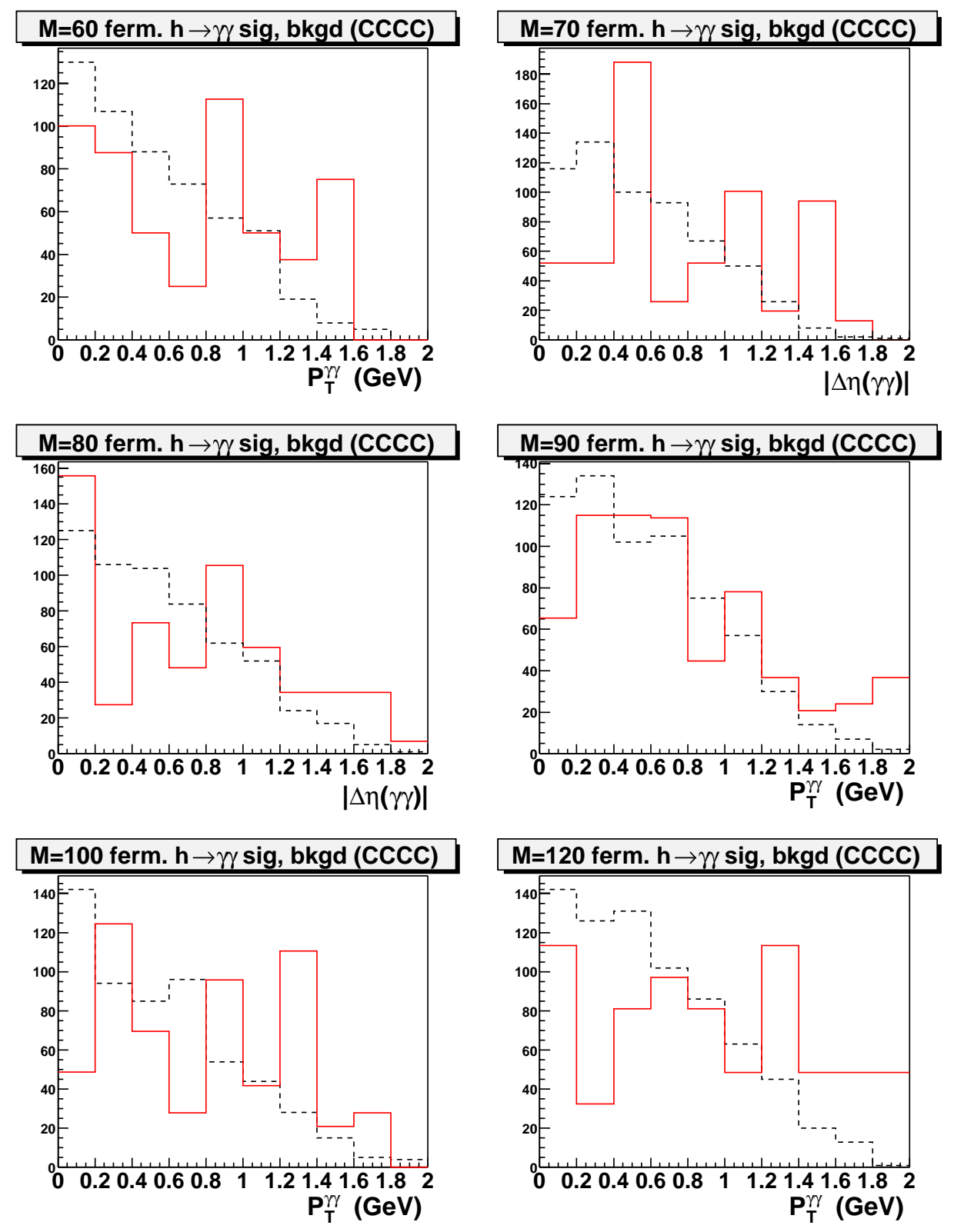

Figure 5.51: Fermiophobic Higgs CCCC $|\Delta \eta(\gamma \gamma)|$ after the $p_{T}^{\gamma \gamma}$ cut. Dashed line - signal, solid line - background. 

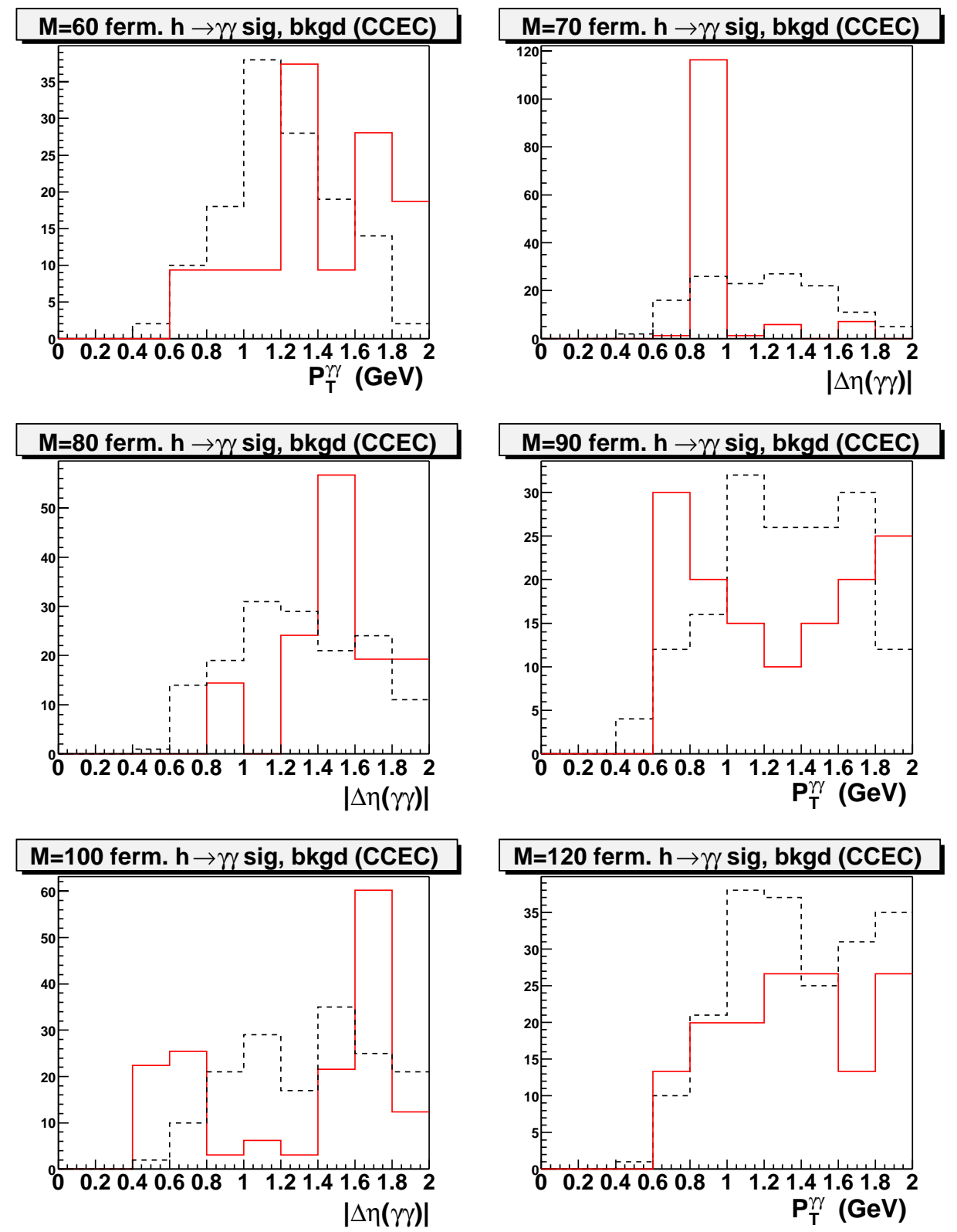

Figure 5.52: Fermiophobic Higgs CCEC $|\Delta \eta(\gamma \gamma)|$ after the $p_{T}^{\gamma \gamma}$ cut. Dashed line - signal, solid line - background. 

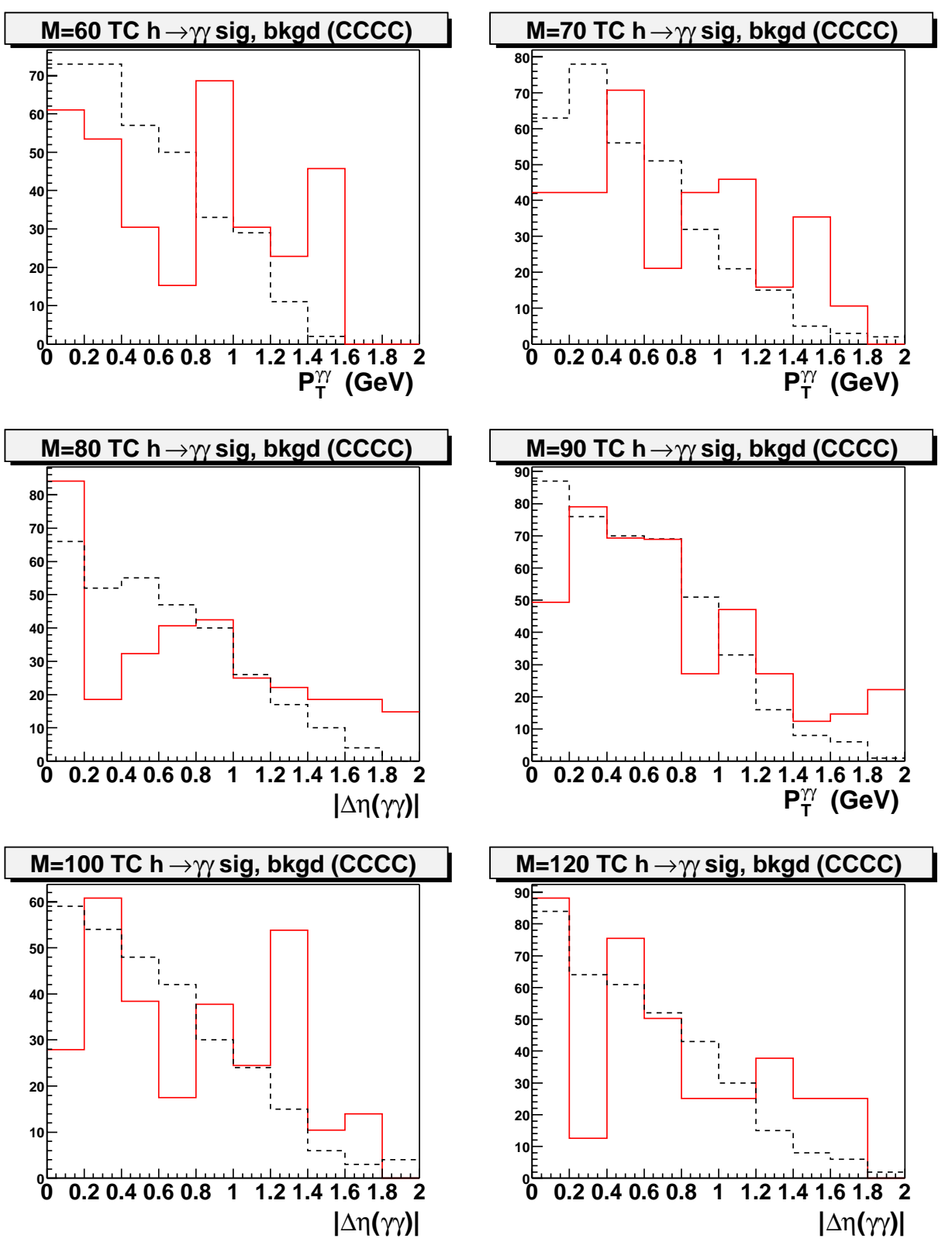

Figure 5.53: Topcolor Higgs CCCC $|\Delta \eta(\gamma \gamma)|$ after the $p_{T}^{\gamma \gamma}$ cut. Dashed line - signal, solid line background.

Another variable that is not correlated with $p_{T}^{\gamma \gamma}$ for higher values of $p_{T}^{\gamma \gamma}$ is energy asymmetry (the bottom plot of Fig. 5.50). Still another interesting variable to look at is the absolute value of the cosine of the helicity angle $\left|\cos \theta^{*}\right|$, mentioned in subsection 5.10.1 and shown in Fig. 5.55.

The signal distribution is expected to have a tendency to be flat. Angular distributions of the spin zero particle decay products are spherically symmetric [99]. However, the acceptance cuts may 

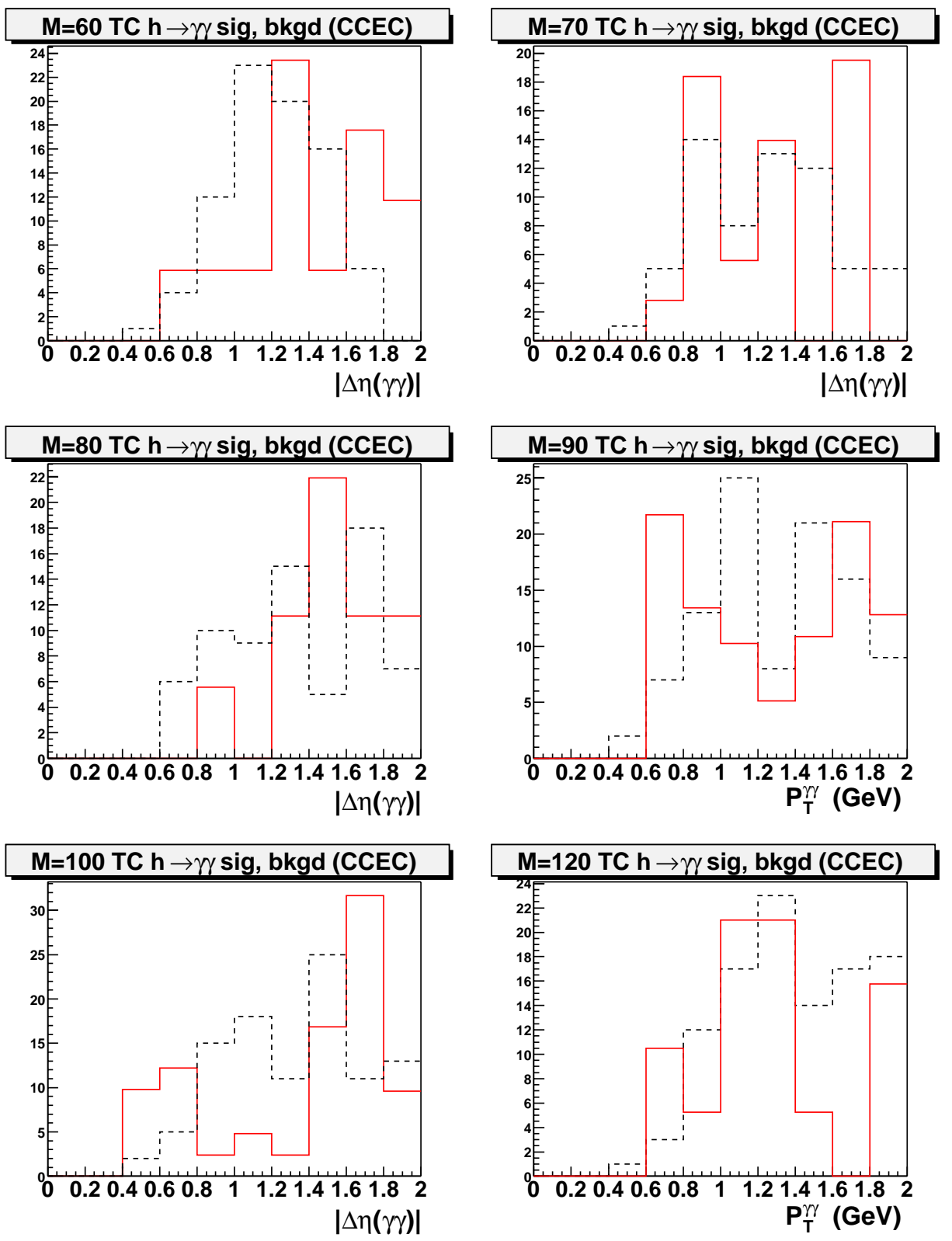

Figure 5.54: Topcolor Higgs CCEC $|\Delta \eta(\gamma \gamma)|$ after the $p_{T}^{\gamma \gamma}$ cut. Dashed line - signal, solid line background. 


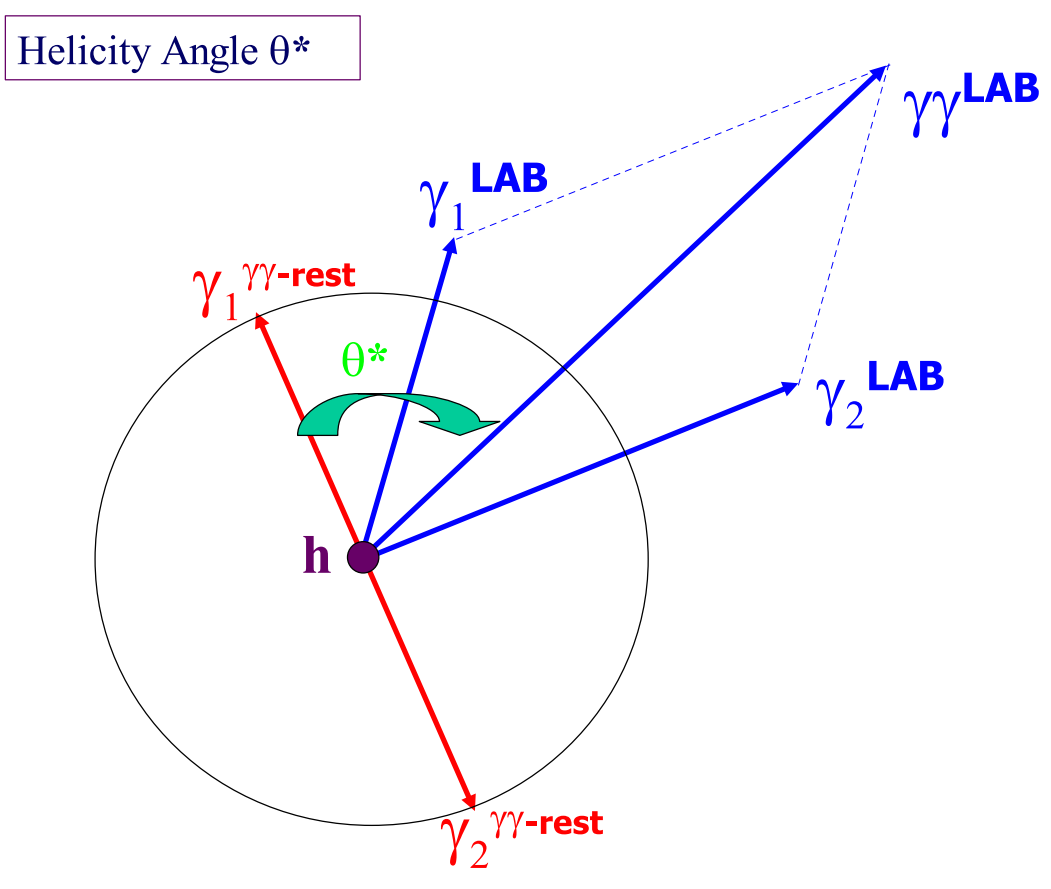

Figure 5.55: Helicity angle: angle between the direction of a photon momentum in the $\gamma \gamma$ rest frame and the diphoton momentum in the lab frame.

modify the flat distribution slightly. The generator level $\left|\cos \theta^{*}\right|$ distribution for the $90 \mathrm{GeV}$ Higgs is shown in Fig. 5.56.

The background distributions are expected to have more complicated structures resulting in peaks at certain regions. Studying this variable, we need to keep in mind that it is correlated with $(E 1-E 2) /(E 1+E 2)$ via

$$
\cos \theta^{*}=\frac{(E 1-E 2)}{\sqrt{(E 1+E 2)^{2}-m^{2}}}
$$

First we try to understand the correlation of these variables with $p_{T}^{\gamma \gamma}$ and see if we could benefit from the $2 \mathrm{D}$ cuts.

$$
(E 1-E 2) /(E 1+E 2) \text { vs. } p_{T}^{\gamma \gamma} \text { distributions are shown in Figures 5.57, 5.58, 5.59, and } 5.60 \text { for }
$$
Fermiophobic Higgs (CCCC), Fermiophobic Higgs (CCEC), Topcolor Higgs (CCCC), and Topcolor Higgs (CCEC), respectively. 


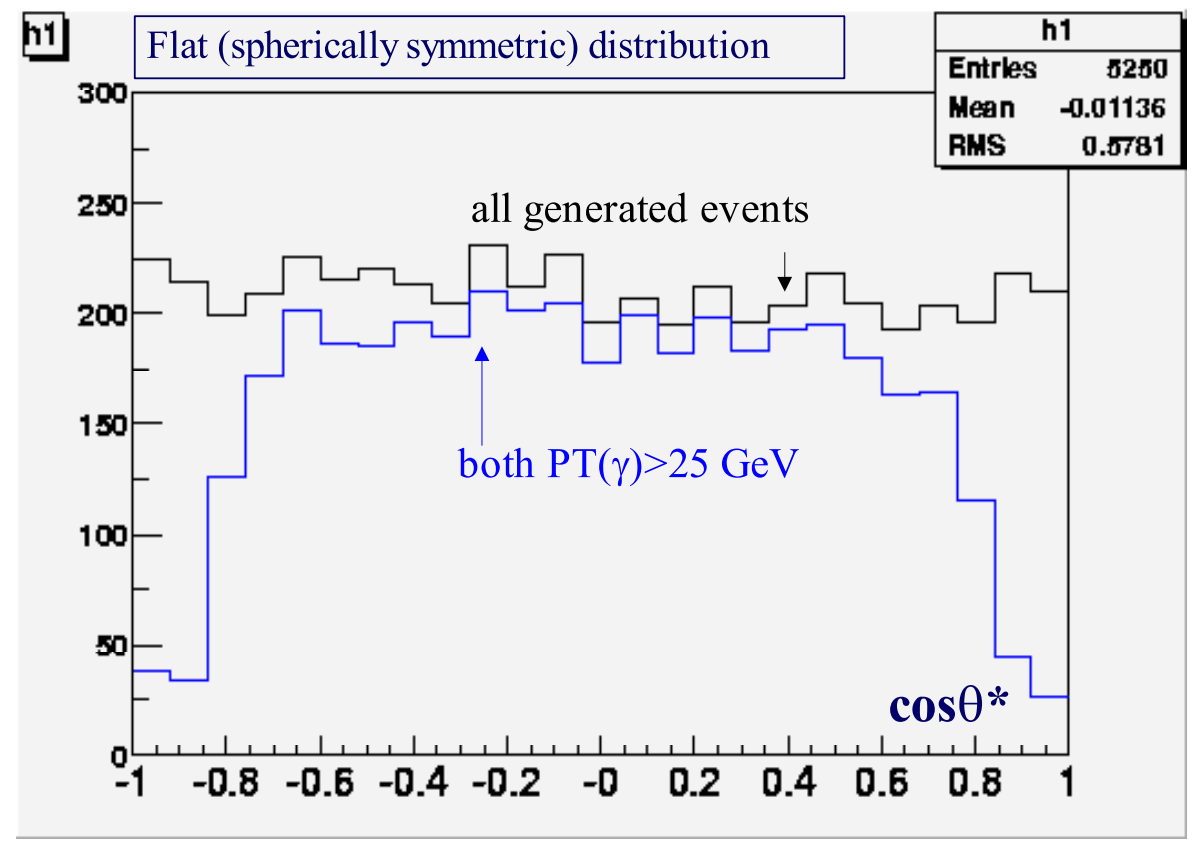

Figure 5.56: $\left|\cos \theta^{*}\right|$ generator-level distribution for $90 \mathrm{GeV}$ Higgs. Top - all events, bottom - after $p_{T}>25 \mathrm{GeV}$ is required for both photons. 

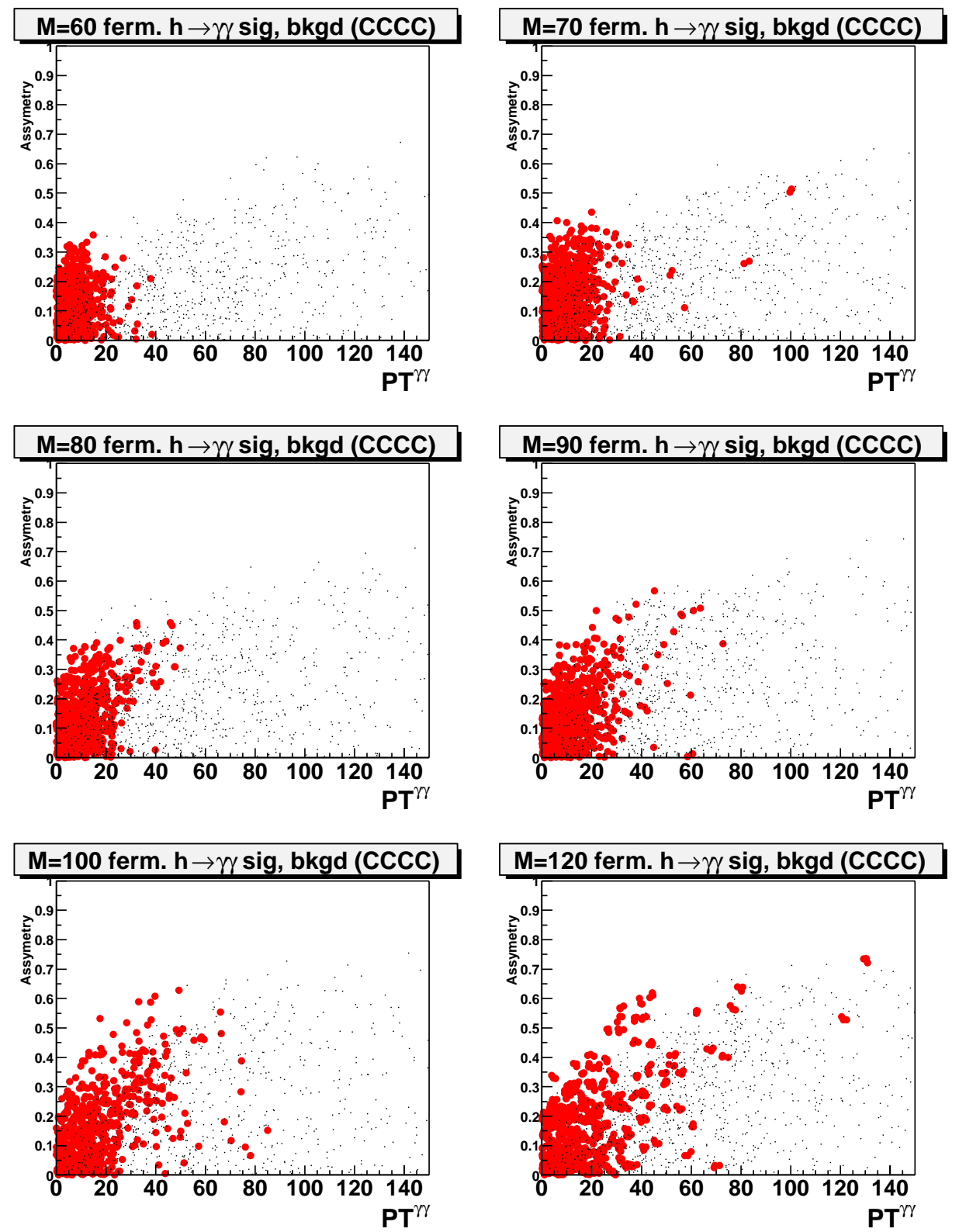

Figure 5.57: Fermiophobic Higgs CCCC $(E 1-E 2) /(E 1+E 2)$ vs. $p_{T}^{\gamma \gamma}$. Small dots: signal, large dots: background. 

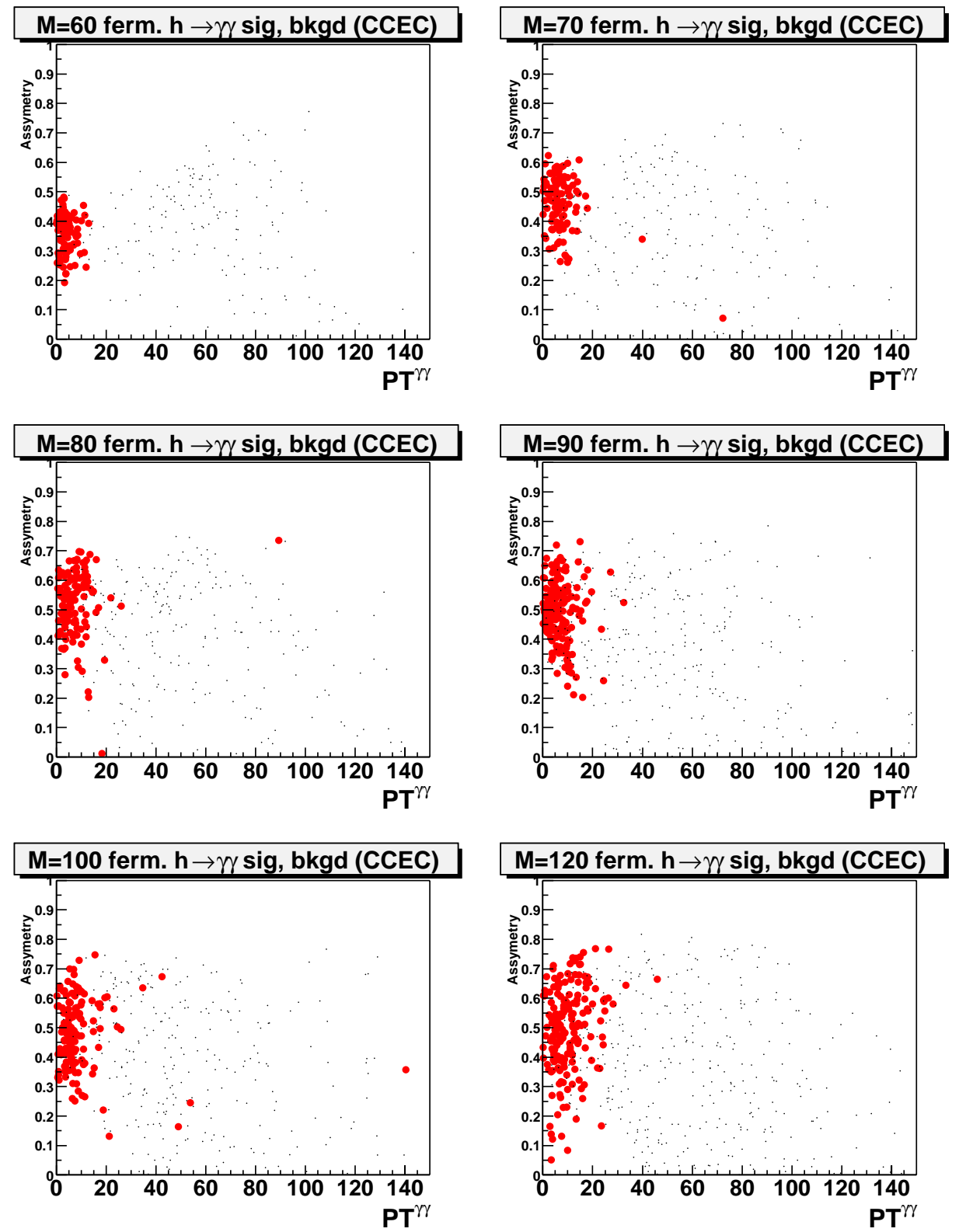

Figure 5.58: Fermiophobic Higgs CCEC $(E 1-E 2) /(E 1+E 2)$ vs. $p_{T}^{\gamma \gamma}$. Small dots: signal, large dots: background. 

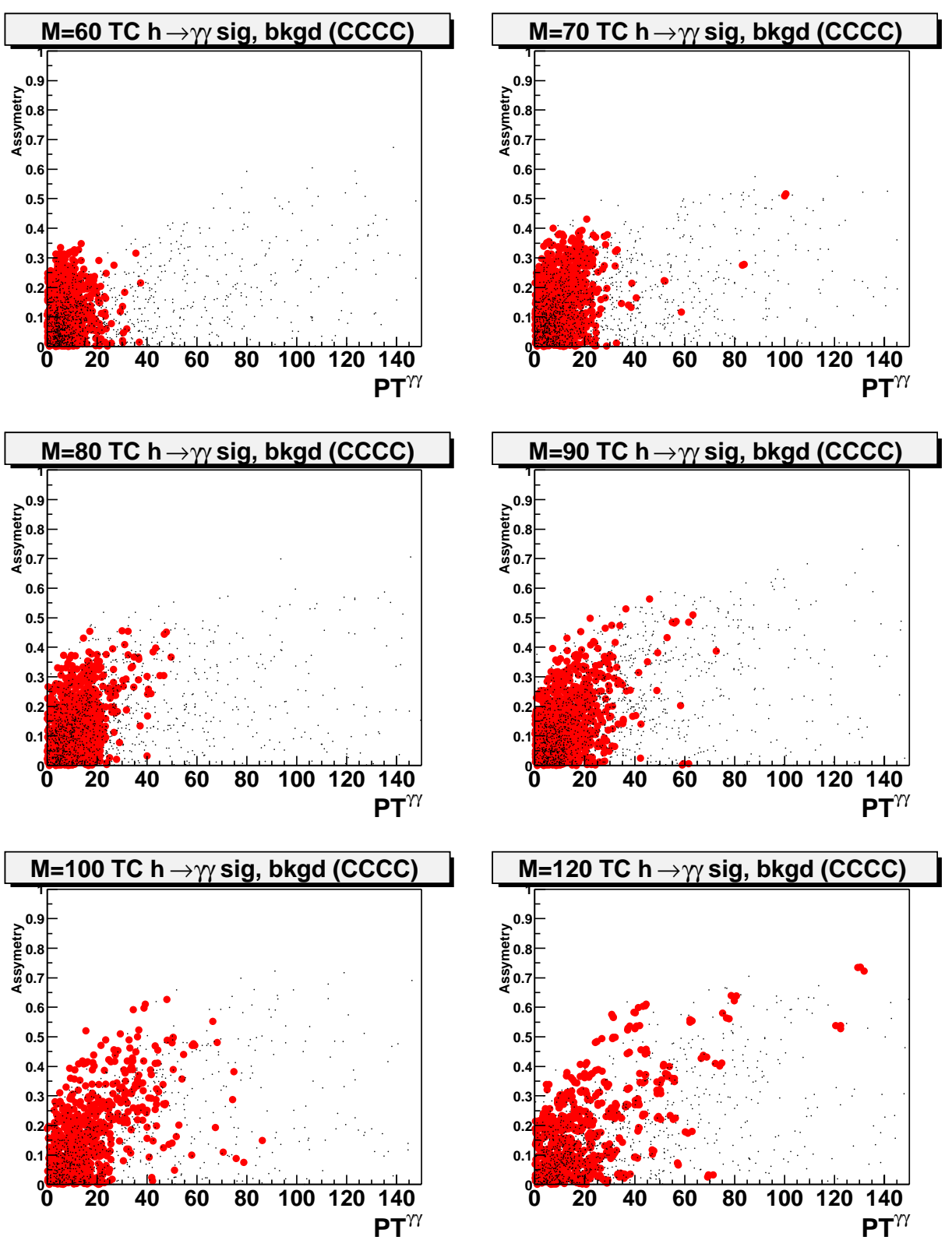

Figure 5.59: Topcolor Higgs CCCC $(E 1-E 2) /(E 1+E 2)$ vs. $p_{T}^{\gamma \gamma}$. Small dots: signal, large dots: background.

$\left|\cos \theta^{*}\right|$ vs. $p_{T}^{\gamma \gamma}$ distributions are shown in Figures 5.61, 5.62, 5.63, and 5.64 for Fermiophobic Higgs (CCCC), Fermiophobic Higgs (CCEC), Topcolor Higgs (CCCC), and Topcolor Higgs (CCEC), respectively. 

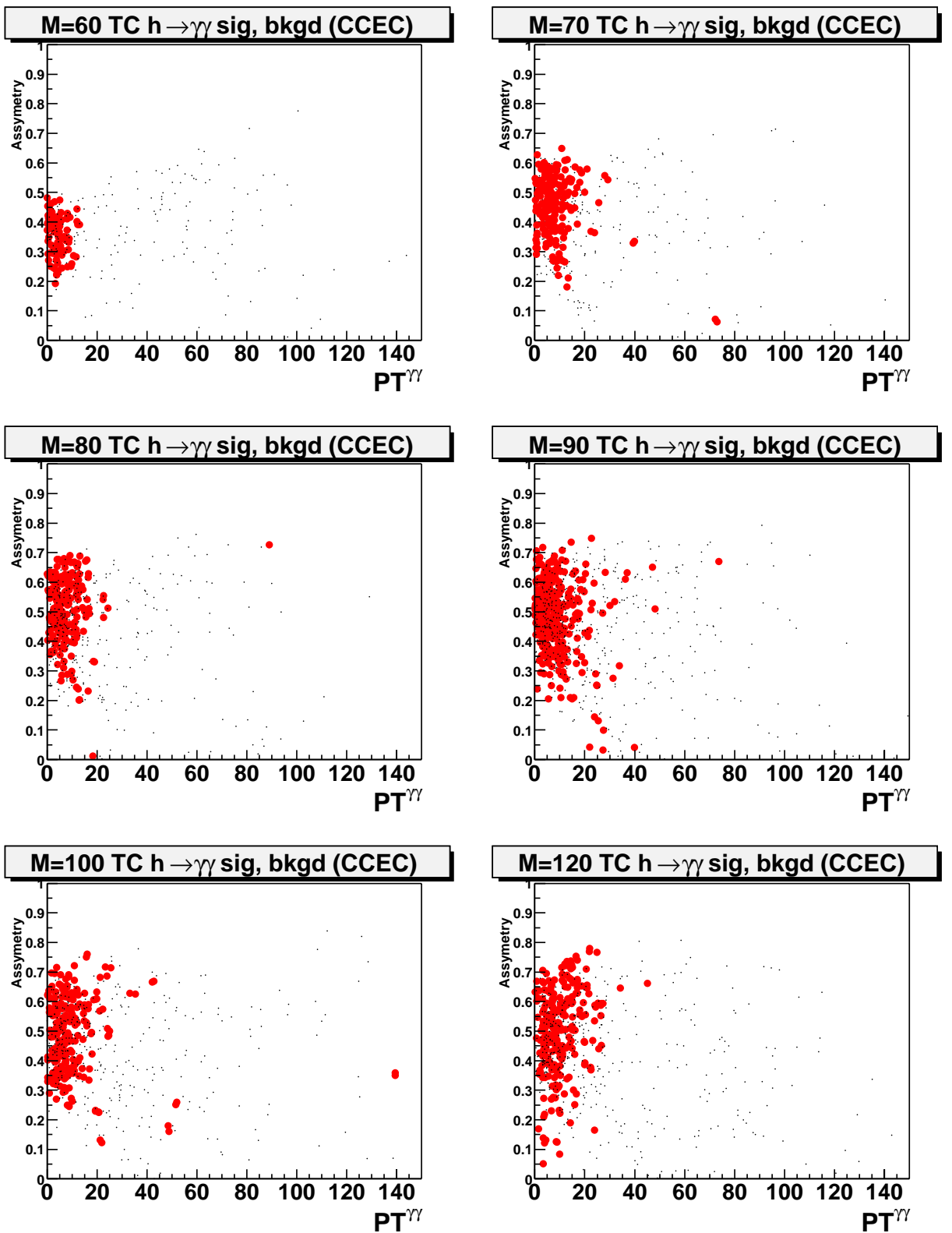

Figure 5.60: Topcolor Higgs CCEC $(E 1-E 2) /(E 1+E 2)$ vs. $p_{T}^{\gamma \gamma}$. Small dots: signal, large dots: background. 

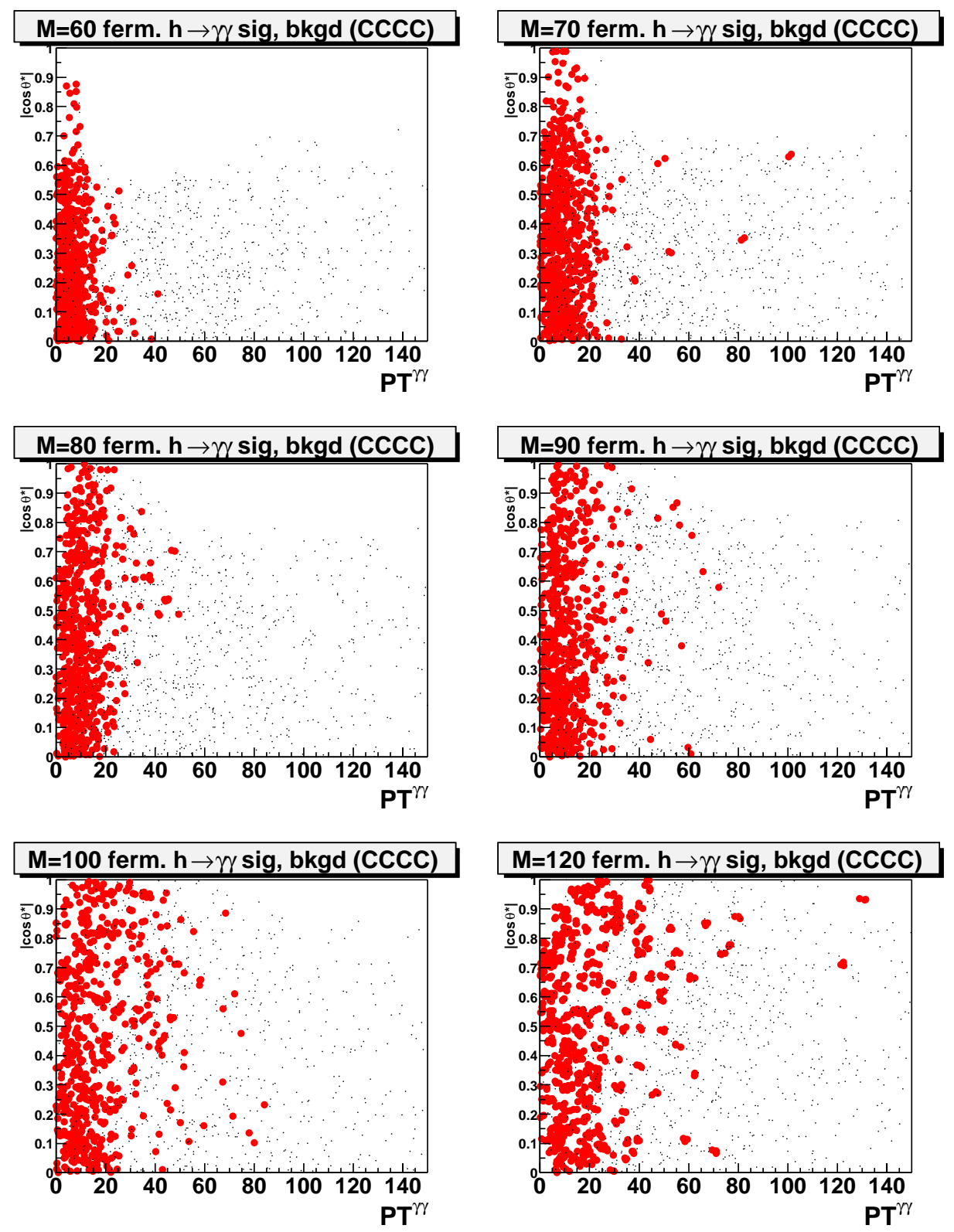

Figure 5.61: Fermiophobic Higgs CCCC $\left|\cos \theta^{*}\right|$ vs. $p_{T}^{\gamma \gamma}$. Small dots: signal, large dots: background. 

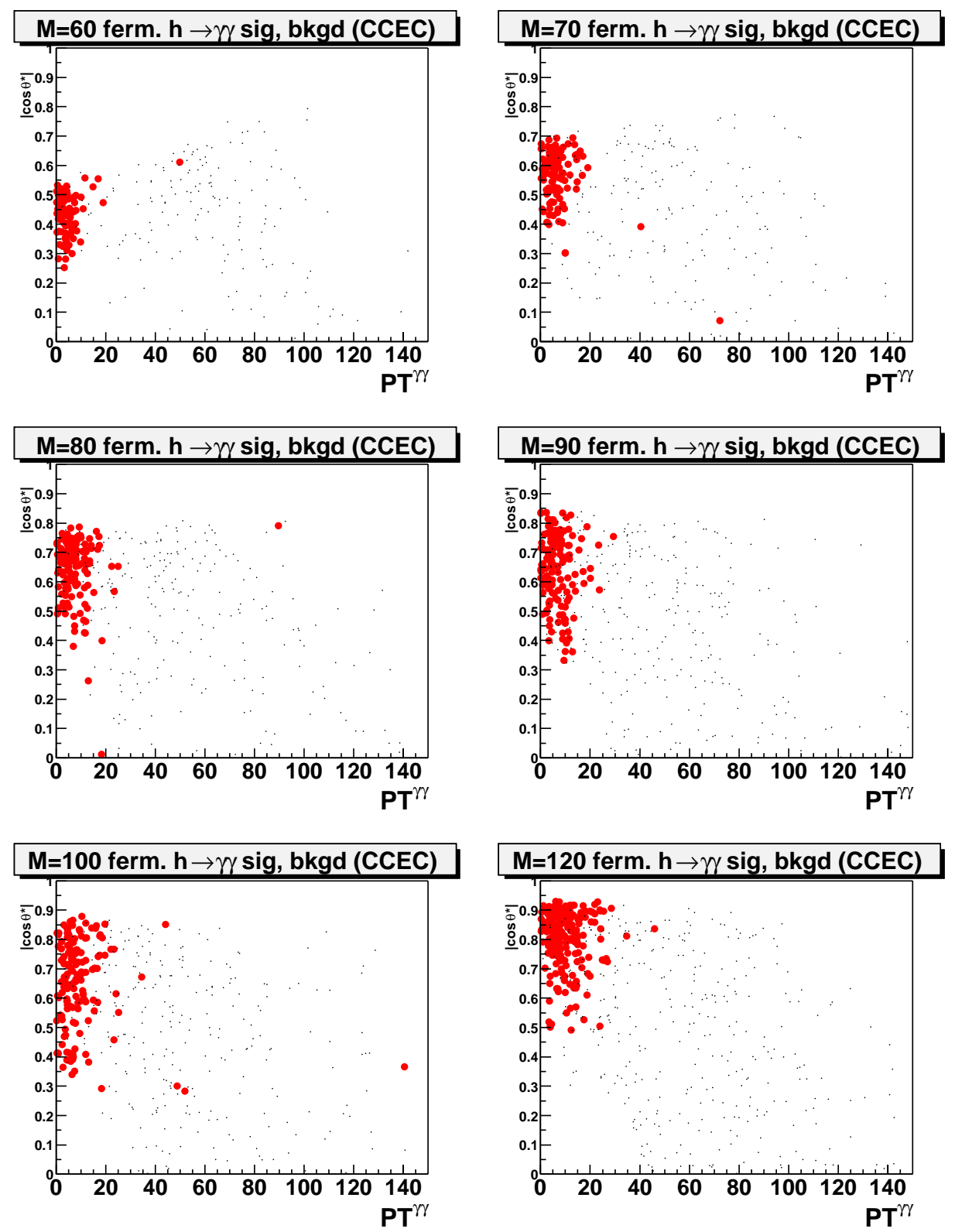

Figure 5.62: Fermiophobic Higgs CCEC $\left|\cos \theta^{*}\right|$ vs. $p_{T}^{\gamma \gamma}$. Small dots: signal, large dots: background. 

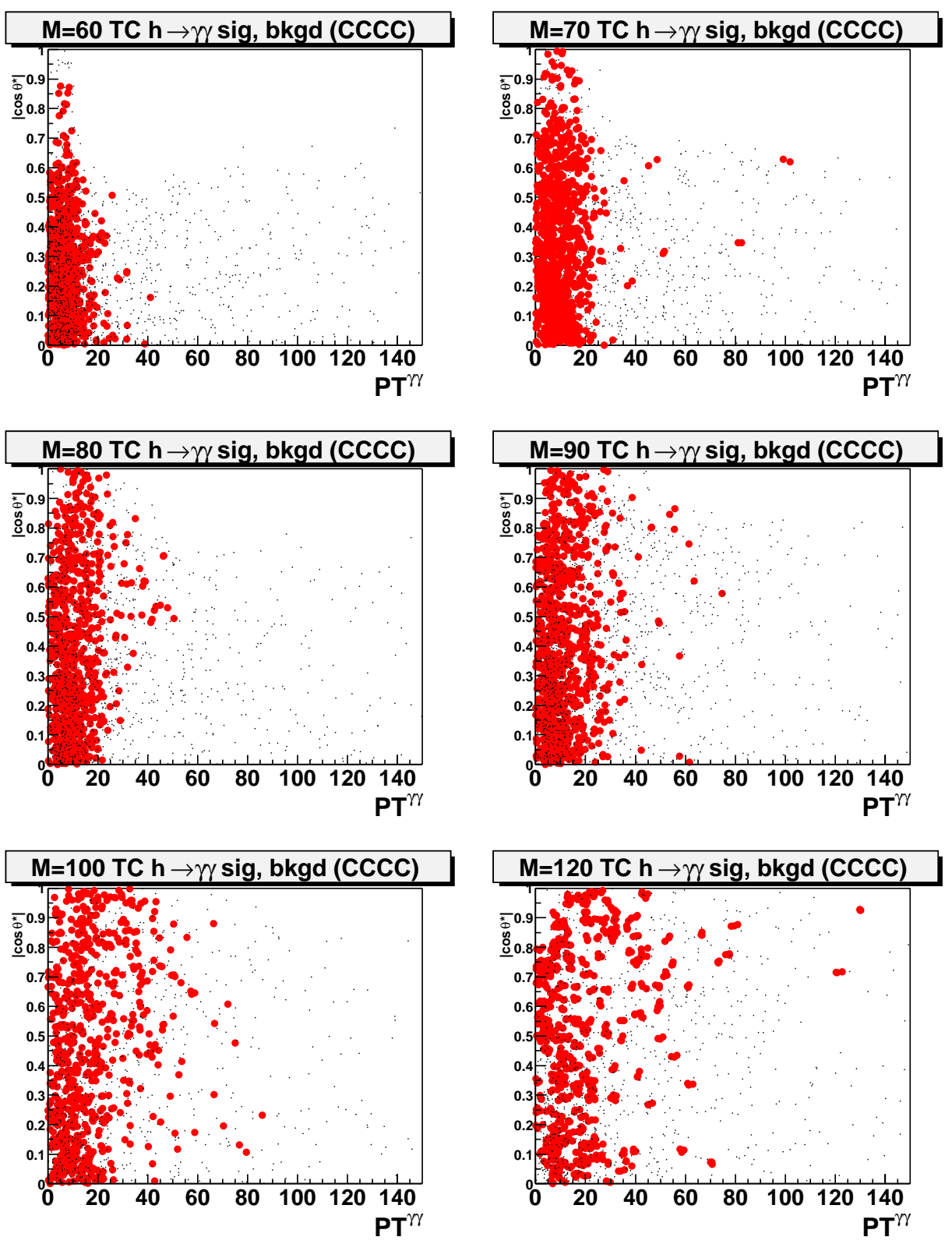

Figure 5.63: Topcolor Higgs CCCC $\left|\cos \theta^{*}\right|$ vs. $p_{T}^{\gamma \gamma}$. Small dots: signal, large dots: background.

These plots do not suggest any significant improvements from 2D-cuts with any of the two variables combined with $p_{T}^{\gamma \gamma}$ in comparison with a single variable $p_{T}^{\gamma \gamma}$ cut.

\subsubsection{Neural Network Studies}

Another possibility we explore, is combining these variables in the neural network. Neural network is a parallel infromation processing structure commonly used for pattern recognition [97]. A neural 

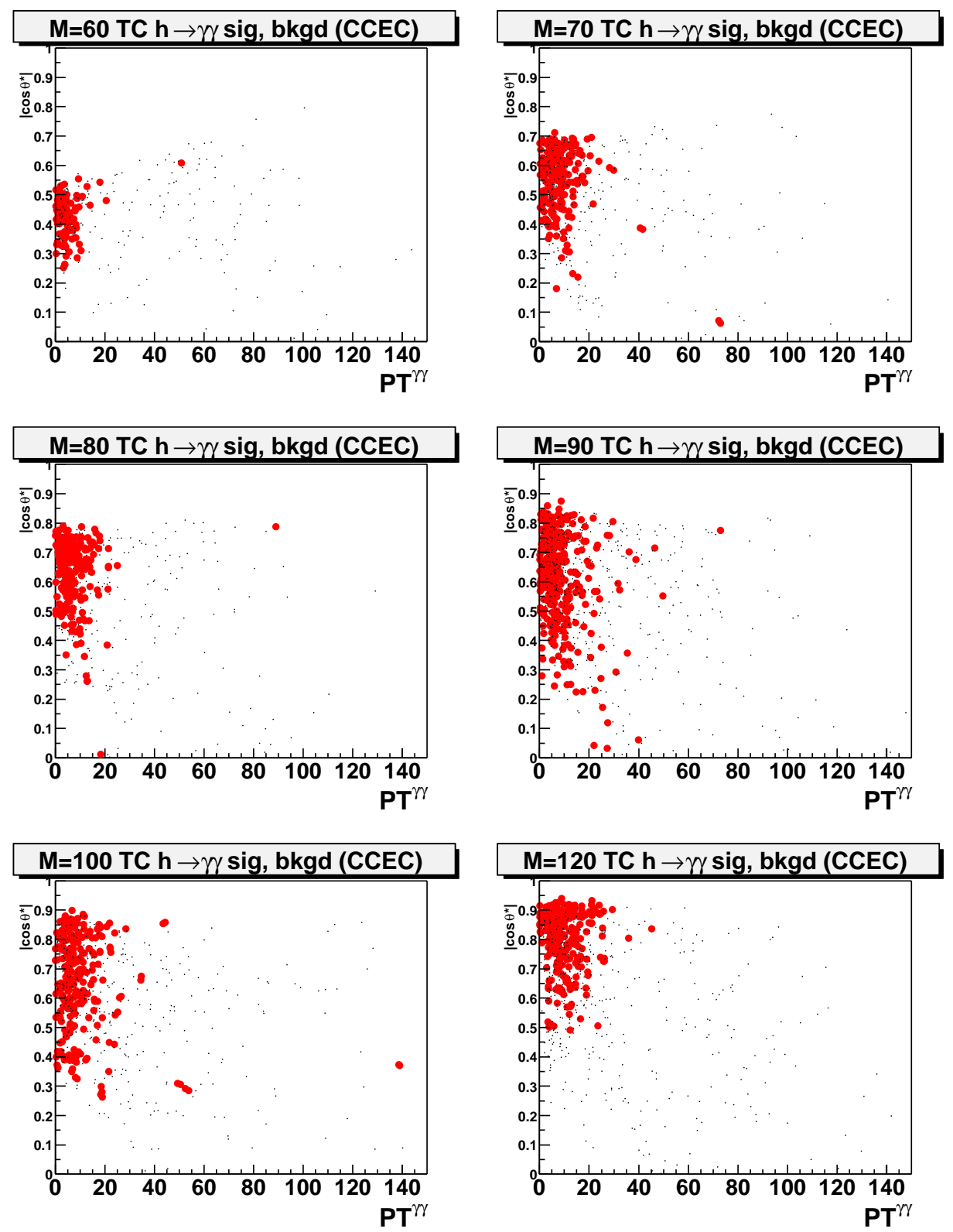

Figure 5.64: Topcolor Higgs CCEC $\left|\cos \theta^{*}\right|$ vs. $p_{T}^{\gamma \gamma}$. Small dots: signal, large dots: background. 
net is a mathematical abstraction of some basic properties of biological nervous systems [59]. It consists of nodes, which exchange information. These nodes are called artificial neurons. Each node $i$ receives input signals $x_{i}$ from other nodes; each input $x_{j}$ is multiplied by a weight $w_{i j}$. The total input of the node is the sum of all weighted input signals minus a threshold $\alpha_{i}$. Applying a transfer function $g(x)$ to the total input gives the output signal $y_{i}$ of node $i$.

$$
y_{i}=g\left(\sum_{j=1}^{n} w_{i j} x_{j}-\alpha_{i}\right)
$$

In this simplest form the transfer function is a step function that gives an output of 1 if the weighted sum of the inputs is above the threshold and -1 otherwise. Frequently one uses continuous transfer functions like $g(x)=\tanh (x)$ which change smoothly from -1 to 1 in the vicinity of $x=0$. The output of a node is often connected to other nodes. If a node connected neither to the sensory input nor to the output it is called a hidden node. If the nodes are connected only in a non-recursive way one speaks about a feed-forward network (Fig. 5.65). Finding the best weights or thresholds for the approximation of a particular function is called learing or training of the network. In order to achieve this goal one needs a training sample, consisting of a set of input vectors and their corresponding function values or output vectors.

We use Root_Jetnet ROOT interface [98] to a FORTRAN JETNET package [100], which uses feed-forward neural network. We consider the following configurations of input variables :

- $p_{T}^{\gamma \gamma},\left|\cos \theta^{*}\right|,(E 1-E 2) /(E 1+E 2)$

- $p_{T}^{\gamma \gamma},\left|\cos \theta^{*}\right|$

- $p_{T}^{\gamma \gamma},(E 1-E 2) /(E 1+E 2)$.

Significance as a function of the NN output is shown in Fig. 5.66 for Fermiphobic Higgs. The comparison with a single variable $p_{T}^{\gamma \gamma}$ cut in Fig. 5.66 is in favor of the latter.

An example plot of NN output is shown in Fig. 5.67 for $\mathrm{M}=60 \mathrm{GeV}$ Fermiophobic Higgs for $p_{T}^{\gamma \gamma}$ ,$\left|\cos \theta^{*}\right|$ input variables.

These results were obtained using a hidden layer that consists of one node. We compare this 


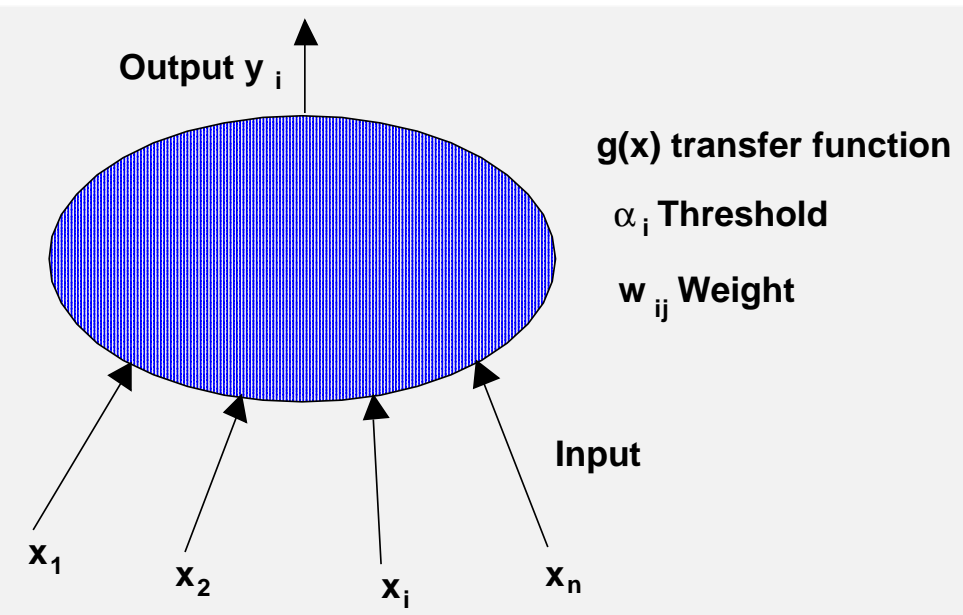

\section{Output information}

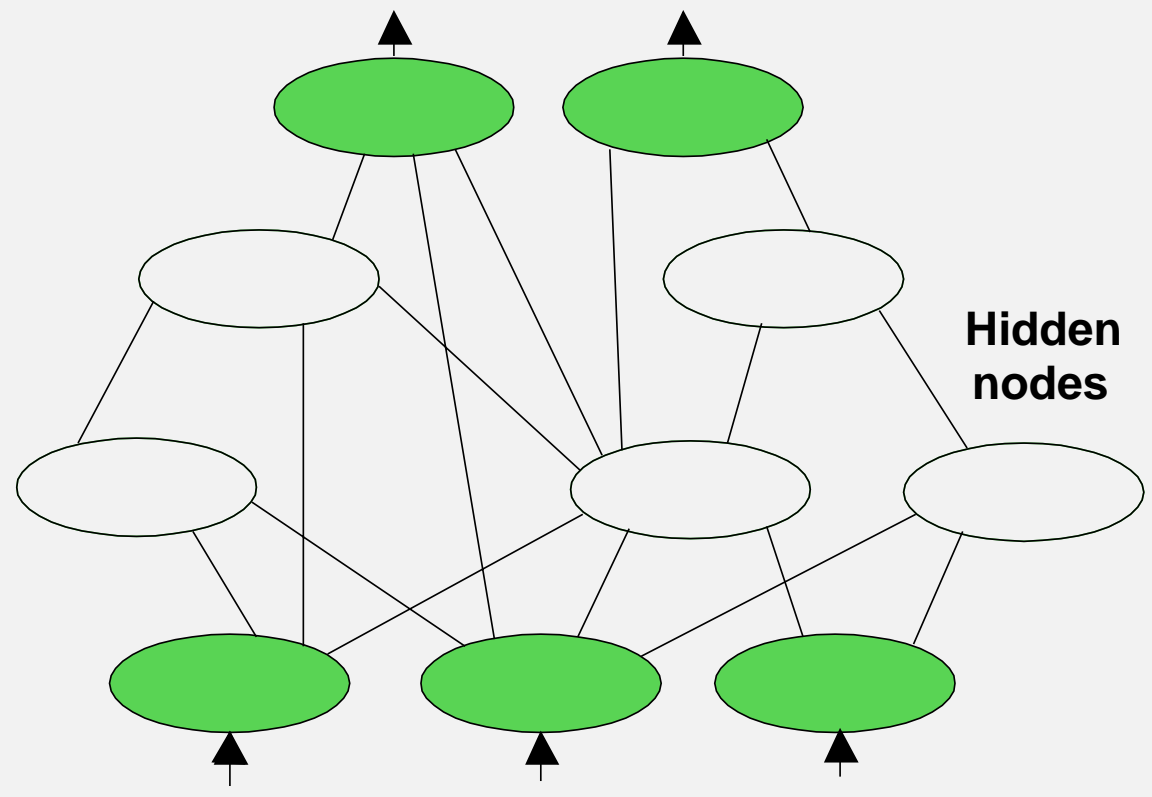

Input information

Figure 5.65: Functional description of a node within a neural network is shown in the top diagram. The input $x_{i}$ is multiplied by the weight $w_{i j}$ and summed up. By applying a threshlod $\alpha_{i}$ and a transfer function $g(x)$ the output is computed. In the bottom diagram one can see a general neural net with input, hidden and output nodes. 

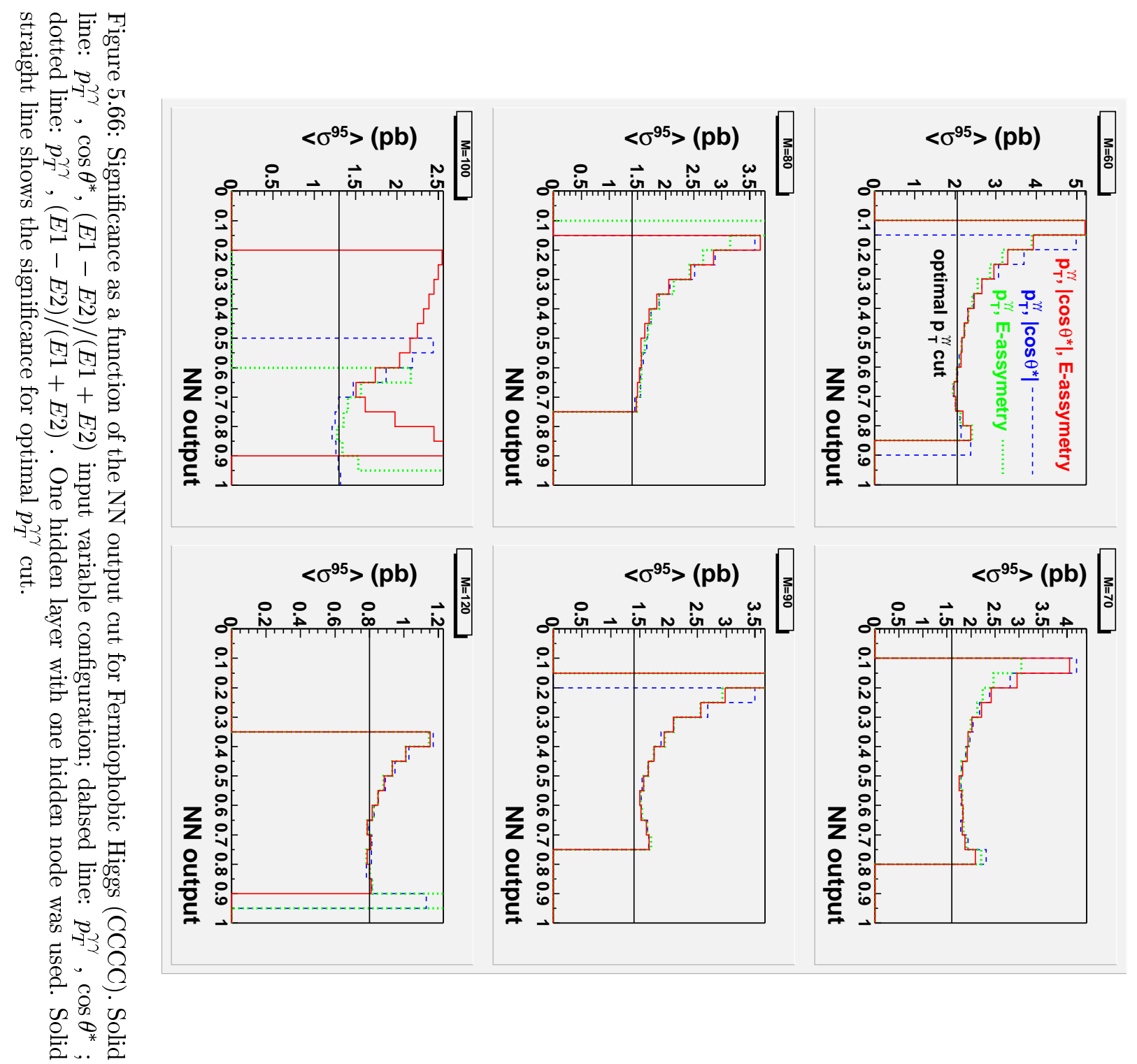


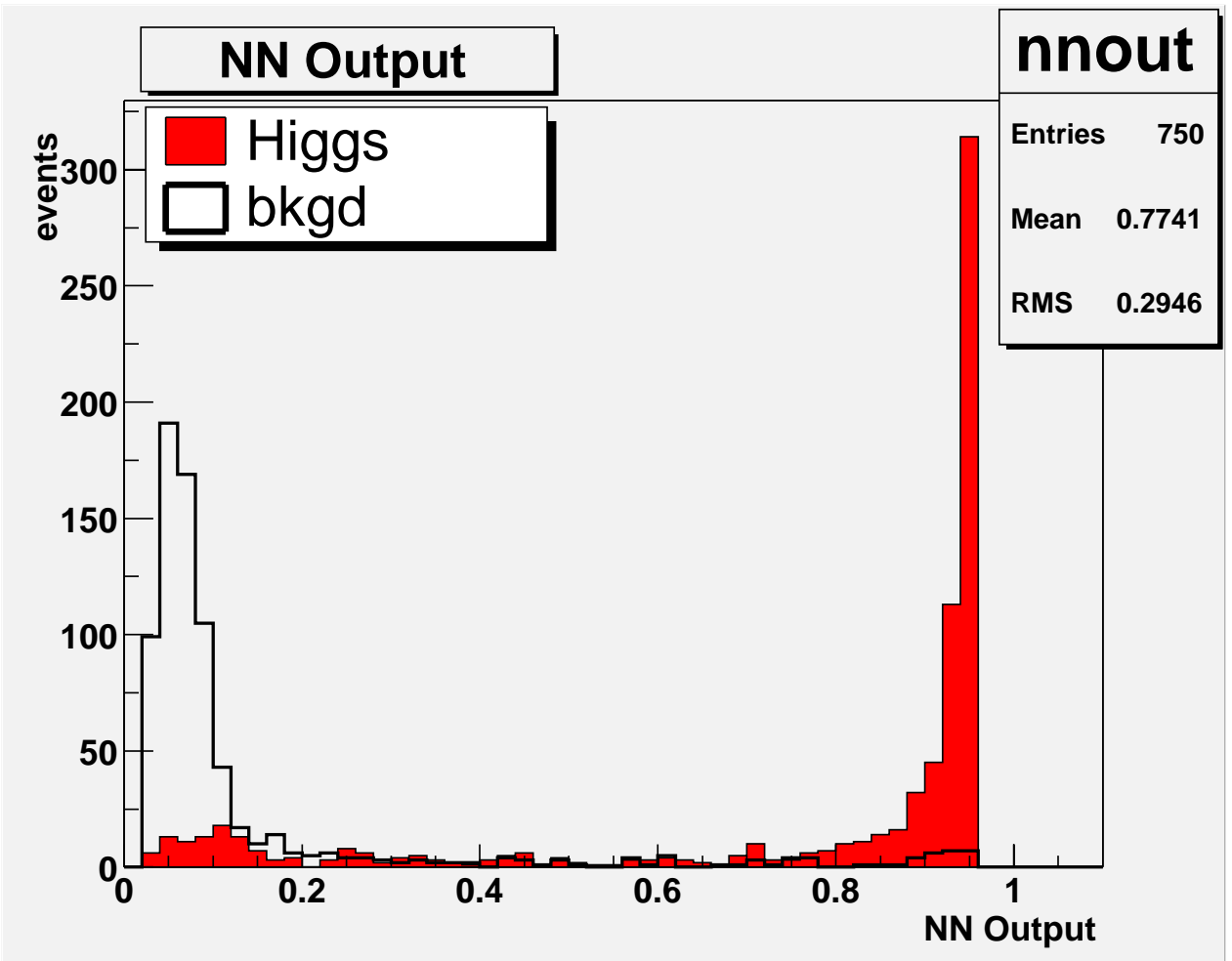

Figure 5.67: Neural Network output for signal and background for the case of $\mathrm{M}=60 \mathrm{GeV}$ Fermiophobic Higgs (CCCC). Filled histogram: signal; Open histogram: background.

with 3 and 5 hidden node configurations in Figure 5.68. No noticeable difference is observed.

It is illustrative to see the NN output distribution patterns in the $p_{T}^{\gamma \gamma}-\left|\cos \theta^{*}\right|$ plane. Figures 5.69 and 5.70 show this for $\mathrm{M}=60 \mathrm{GeV}$ signal and background, respectively.

No improvement in significance from adding $\left|\cos \theta^{*}\right|$ to $p_{T}^{\gamma \gamma}$ via Neural Net is consistent with the fact that contours of constant NN output tend to be parallel to the $\left|\cos \theta^{*}\right|$ axis. Hence cutting on the NN output is effectively cutting on the $p_{T}^{\gamma \gamma}$. 

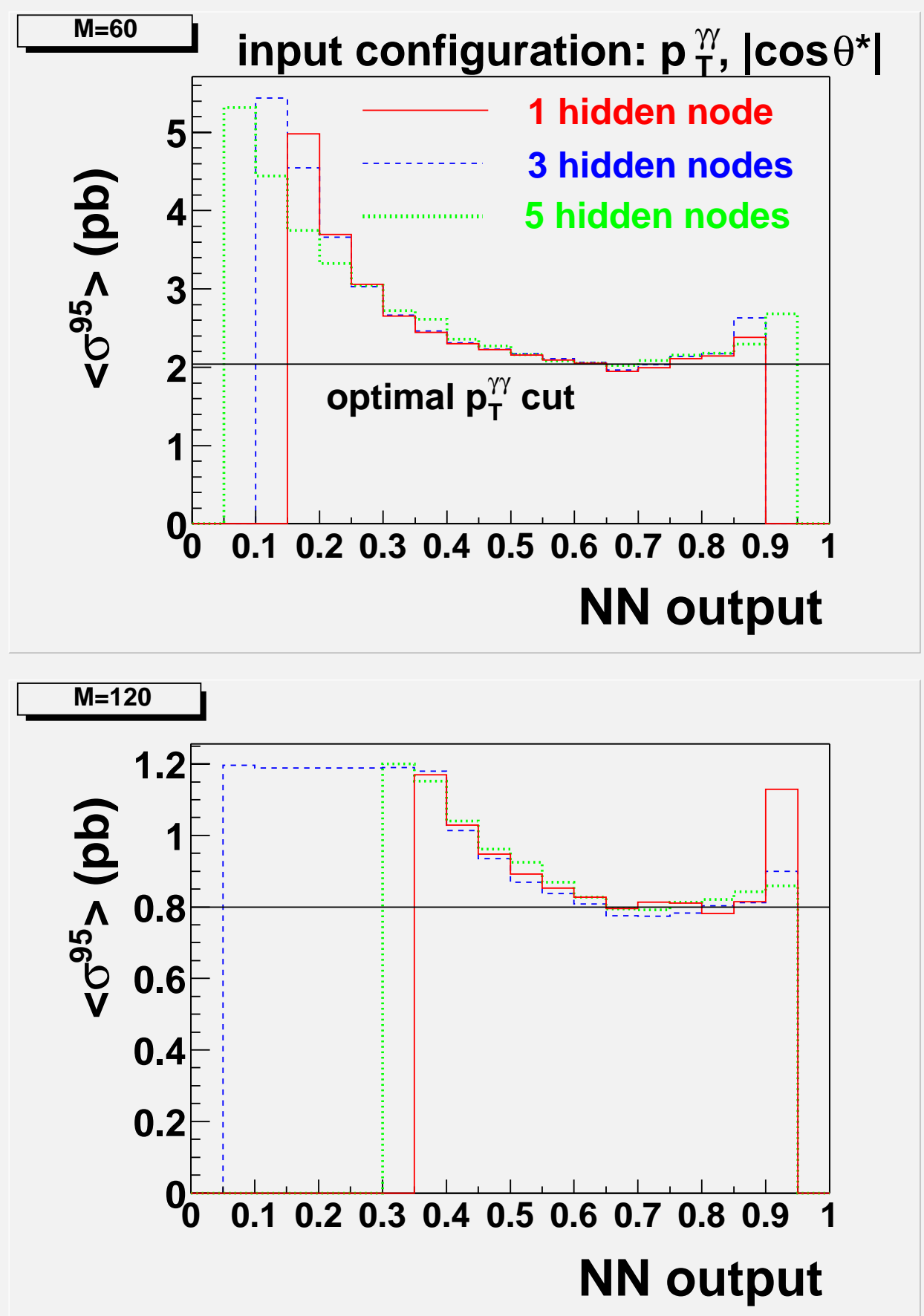

Figure 5.68: Significance as a function of Neural Network output cut for different number of hidden nodes for Fermiophobic Higgs (CCCC). Solid line: 1 hidden node; dashed line: 3 hidden nodes; dotted line : 5 hidden nodes. Input variables are $p_{T}^{\gamma \gamma}$ and $\cos \theta^{*}$. Top plot corresponds to $\mathrm{M}=60$ $\mathrm{GeV}$, the bottom shows $\mathrm{M}=120 \mathrm{GeV}$ case. 


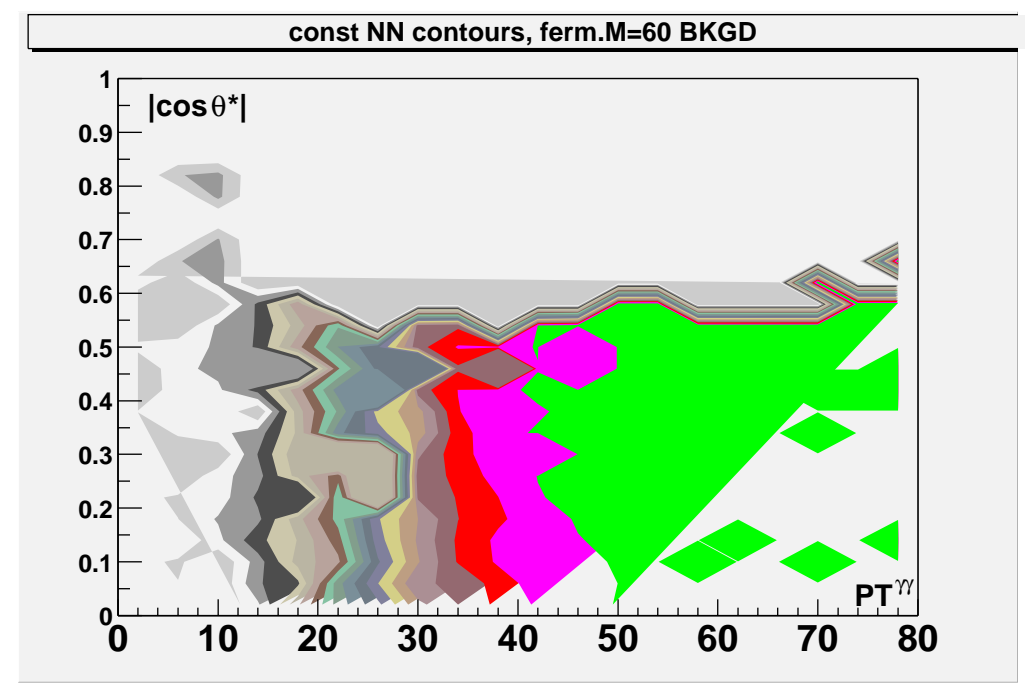

Figure 5.69: Contours of constant $\mathrm{NN}$ output in the $p_{T}^{\gamma \gamma}-\left|\cos \theta^{*}\right|$ plane for $\mathrm{M}=60 \mathrm{GeV}$ Fermiophobic Higgs signal (CCCC).

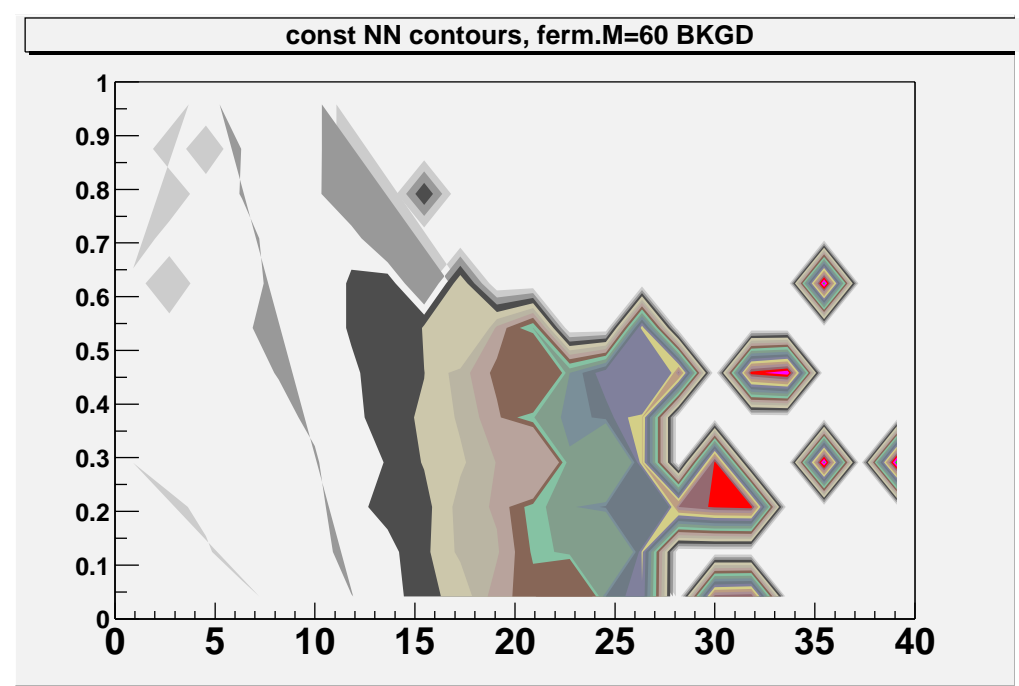

Figure 5.70: Contours of constant NN output in the $p_{T}^{\gamma \gamma}-\left|\cos \theta^{*}\right|$ plane for the CCCC background in the window centered at $\mathrm{M}=60 \mathrm{GeV}$. 


\subsubsection{Conclusion on Optimization}

Single optimization variable $p_{T}^{\gamma \gamma}$ turns out to be sufficient for this analysis. The values of the cuts for different subsamples and corresponding event counts are listed in Section 5.10.3. Including jet

objects in this analysis is not beneficial. Combining $p_{T}^{\gamma \gamma}$ with additional kinematic variables either via simple cuts or via Neural Net does not improve the sensitivity. 


\title{
Chapter 6
}

\section{Higgs Cross Section Limits}

\author{
So close, no matter how far... \\ "Metallica"'s song "Nothing else matters"
}

As discussed in Section 5.10.3, no significant excess of events that could be interpreted as Higgs signal is seen in this analysis. The observed $\gamma \gamma$ spectrum is described well by the expected SM backgrounds (see, e.g. Table 5.8). We use the $\gamma \gamma$ data to set the upper limits on Higgs cross-section following the Bayesian approach [101, 102].

\subsection{Bayesian Approach}

\subsubsection{Brief Overview}

There are two major approaches to interpreting the results of experimental data analysis :

- Bayesian approach;

- Classical (Frequentist) approach;

DØ adopted the Bayesian approach which has the following distinctive features:

1. Probabilities are interpreted as numbers whose relative values represent the degree of belief. 
(For comparison, classically, probability is defined as a relative frequency with which an event occurs in a set of experimental (could be hypothetical) trials) ${ }^{1}$

2. Unlike in the classical approach, previous knowledge (or lack thereof) is incoroprated into the analysis of experimental data.

3. Bayes' theorem :

$$
P(X \mid Y)=\frac{P(Y \mid X) \times P(X)}{P(Y)}
$$

where symbol $P(A \mid B)$ denotes conditional probability, i.e. probability of $A$ given that $B$ is true. The theorem allows us to turn the conditional probabilities around. One can make use of this property in data analysis to relate the model that one is testing in the experiment with the observed data:

$$
P(\text { model } \mid \text { data }) \propto P(\text { data } \mid \text { model }) \times P(\text { model })
$$

This expression has the same form when written in terms of probability densities (likelihoods) as well as probabilities (from now on we will use same symbol $P$ to denote probability density). Equation (6.2) in a sense encapsulates the process of learning:

- we start with $P($ model) (called prior) which reflects our state of knowledge before the experiment;

- the observed data is accounted for via $P($ data $\mid$ model $)$ likelihood;

- the outcome of the analysis is $P$ (model $\mid$ data) (called posterior probability density or simply likelihood) from which we derive our conclusions as to the plausibility of the tested model.

The denominator factor in (6.2) was left out on purpose. It contributes only to the overall normalization but not to the shape of the likelihood and can be omitted footnotemark.

\footnotetext{
${ }^{1}$ Note that the distinction is in the interpretation of the meaning of probability, not in its axiomatic definition. Both approaches rely on the same formal theory of probability.

${ }^{1}$ This factor can be ignored in case of parameter estimation problems. However, in some situations, such as model selection it is crucial.
} 


\subsection{2 $h \rightarrow \gamma \gamma$ applications}

For the case of new particle searches [102] there is only one accepted model ${ }^{2}$ : we observe $N$ events that come from signal $S$ ( $h \rightarrow \gamma \gamma$ events in our case) and the background $B$.

$$
N=S+B
$$

The number of signal events $S$ is related to the cross-section $\sigma$, detector and ID efficiency $\epsilon$, and integrated luminosity $\mathcal{L}$ as:

$$
N=\mathcal{L} \epsilon \sigma
$$

Hence

$$
N=\mathcal{L} \epsilon \sigma+B
$$

In this expression $\epsilon$ denotes the product of all the efficiencies and acceptances mentioned in the previous Chapter; $\mathrm{B}(\gamma \gamma)=1$ assumption is made at this stage of the analysis, i.e $\sigma$ stands for the production cross section.

The Bayes theorem for our problem becomes:

$$
P(\sigma, \mathcal{L}, \epsilon ; B \mid N)=P(N \mid \sigma, \mathcal{L}, \epsilon ; B) \times P(\sigma, \mathcal{L}, \epsilon ; B)
$$

We replaced the proportionality with equality following the likelihood shape argument mentioned above. Because our interest is in $\sigma$, and not in $\mathcal{L}, \epsilon$ or $B$, the last three $(\mathcal{L}, \epsilon, B)$ are often called nuisance parameters. To remove any dependence on the nuisance parameters, equation (6.6) is integrated over them. The result is the posterior distribution for $\sigma$ :

$P(\sigma \mid N)=\int_{0}^{\infty} d \mathcal{L} \int_{0}^{1} d \epsilon \int_{0}^{\infty} d B P(\sigma, \mathcal{L}, \epsilon ; B \mid N)=\int_{0}^{\infty} d \mathcal{L} \int_{0}^{1} d \epsilon \int_{0}^{\infty} d B P(N \mid \sigma, \mathcal{L}, \epsilon ; B) \times P(\sigma, \mathcal{L}, \epsilon ; B)$.

Our next step is to write out the explicit form of the integrand in (6.7). It consists of two factors :

\footnotetext{
${ }^{2}$ the discussion is specific to counting experiments
} 
the likelihood $P(N \mid \sigma, \mathcal{L}, \epsilon ; B)$ and the prior $P(\sigma, \mathcal{L}, \epsilon ; B)$.

In the case of counting experiments, conventionally accepted general likelihood function is the Poisson distribution :

$$
P(N \mid \sigma, \mathcal{L}, \epsilon ; B)=\frac{e^{-(\mathcal{L} \epsilon \sigma+B)}(\mathcal{L} \epsilon \sigma+B)^{N}}{N !}
$$

Then the prior must be assigned using available information (such as the knowledge of the integrated luminosity within some bound of uncertainties) about each of the parameters in the problem. In general the prior can contain the correlations between the parameters. Assuming that the correlations between $\sigma, \mathcal{L}, \epsilon$ and $B$ can be neglected $P(\sigma, \mathcal{L}, \epsilon ; B)$ can be factorized into a product of independent priors:

$$
P(\sigma, \mathcal{L}, \epsilon ; B)=P(\sigma) P(\mathcal{L}) P(\epsilon) P(B)
$$

By the signal cross-section prior $P(\sigma)$ we would like to describe the absence of knowledge of $\sigma(p \bar{p} \rightarrow$ $h+X)$ before this experiment. The choice of the form of such prior poses a problem since the theory of "uninformative priors" is the least well-developed portion of Bayesian statistics. We follow the suggestion from [102] and use a flat prior of finite range

$$
P(\sigma)= \begin{cases}1 / \sigma_{\max }, & \text { if } 0 \leq \sigma \leq \sigma_{\max } \\ 0, & \text { otherwise }\end{cases}
$$

where $\sigma_{\max }$ is chosen sufficiently large, so that the integral of the likelihood function for $\sigma>\sigma_{\max }$ is negligible. For practical purposes such choice implies ignoring the $P(\sigma)$ in the calculation of the posterior $P(\sigma \mid N)$, since it has no effect on its shape, and restricting allowed range of $\sigma$ to $\sigma>0$.

All of the remaining priors $P(\mathcal{L}), P(\epsilon)$ and $P(B)$ are given by Gaussian distributions ${ }^{3}$ :

$$
P(x)=\frac{1}{\sqrt{2 \pi} \sigma_{x}} e^{-\left(x-x_{0}\right)^{2} / 2 \sigma_{x}^{2}}
$$


where $x_{0}$ and $\sigma_{x}$ are the mean value and standard deviation of parameter $x=\mathcal{L}, \epsilon, B$.

Combining (6.7), (6.8), (6.9), and (6.10) yields the final expression [91] for the posterior likelihood:

$P(\sigma \mid N)=\int_{0}^{\infty} d \mathcal{L} \int_{0}^{1} d \epsilon \int_{0}^{\infty} d B \frac{e^{-(\mathcal{L} \epsilon \sigma+B)}(\mathcal{L} \epsilon \sigma+B)^{N}}{N !} e^{-\left(\mathcal{L}-\mathcal{L}_{0}\right)^{2} / 2 \sigma_{\mathcal{L}}^{2}} e^{-\left(\epsilon-\epsilon_{0}\right)^{2} / 2 \sigma_{\epsilon}^{2}} e^{-\left(B-B_{0}\right)^{2} / 2 \sigma_{B}^{2}}$

At the computation stage, $\mathcal{L}$ and $\epsilon$ were combined into a single variable (corresponding uncertainties added in quadrature). Integration ranges were optimized to \pm 3 (5) standard deviations around the mean value.

Finally the $95 \%$ Confidence Level (CL) upper bound on the cross-section $\sigma^{95}$ is determined by the (numerical) solution [91] of the following integral equation:

$$
\int_{0}^{\sigma^{95}} P(\sigma \mid N) d \sigma=0.95
$$

\subsection{Results}

Table 6.1 shows $95 \mathrm{CL}$ upper cross-section limits for Fermiophobic Higgs and Topcolor Higgs. The CCCC subsample limits are calculated with and without the optimal $p_{T}^{\gamma \gamma}$ cut. The limits are calculated for the CCEC subsample as well. Final results are obtained via combining CCCC and CCEC likelihoods ${ }^{4}$ for the case when the optimal $p_{T}^{\gamma \gamma}$ cut is applied. Comparison is made with the NLO theoretical predictions [24, 25].

Figures 6.1 and 6.2 illustrate how combining (multiplying) the likelihoods of the two subsamples affects the limit. Figure 6.1 describes the situation when the limit is improved whereas Fig. 6.2 shows the opposite situation. Individual likelihood shapes and combined results are explained in terms of relationship between quantities that enter the posterior likelihood equation (6.11).

\footnotetext{
${ }^{3}$ Often (but not necessarily always!) when there is an estimate of both the value and uncertainty in a given quantity the appropriate distribution for a prior is a Gaussian [102].

${ }^{4}$ The two likelihoods are multiplied. Common systematic error is taken out of one of the likelihoods, otherwise it would be counted twice. We take luminosity error (the dominant source of the systematic error) out of the CCEC likelihood.
} 

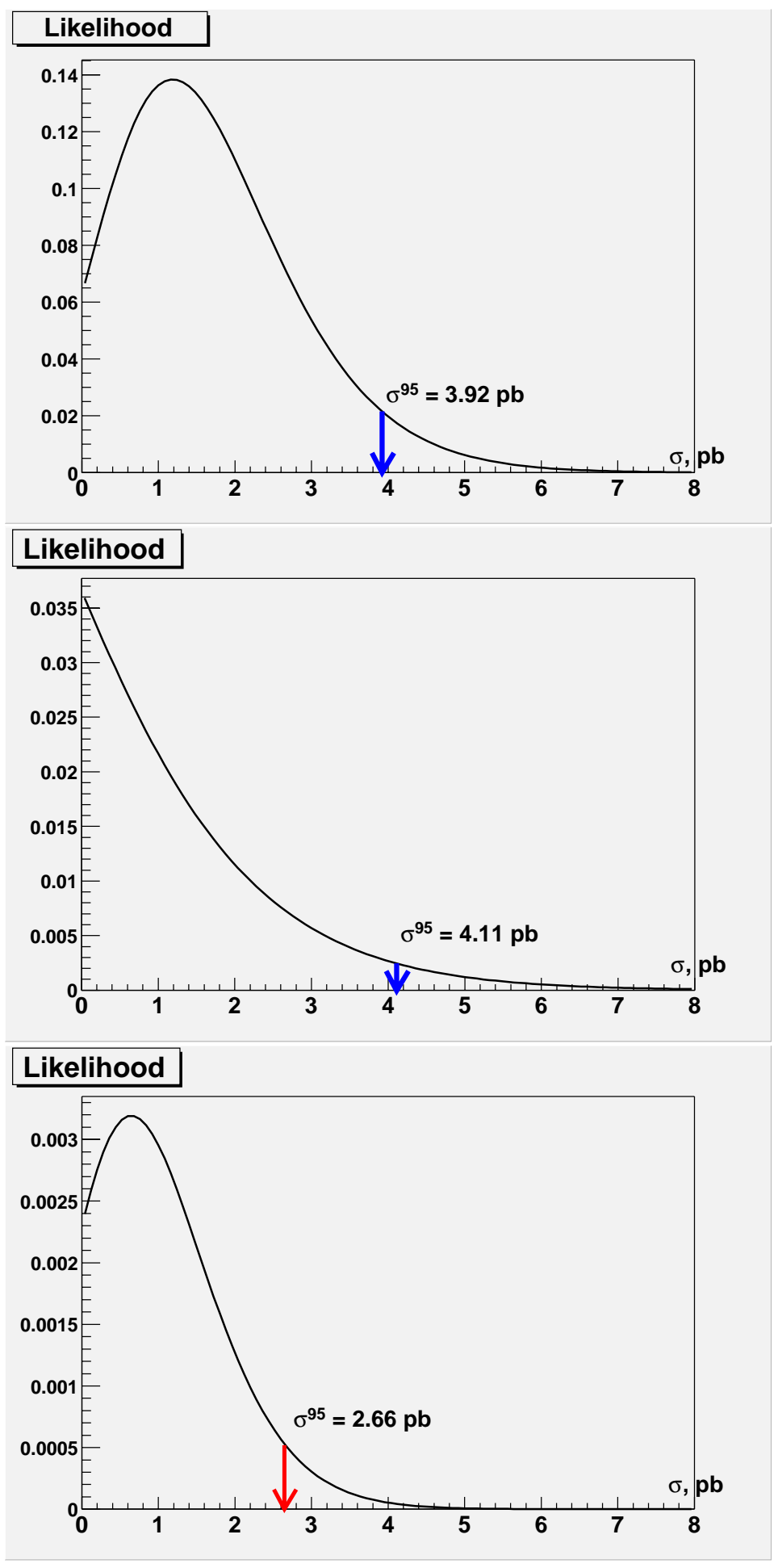

Figure 6.1: Improving the limit via combining likelihoods (example for $\mathrm{M}=100 \mathrm{GeV}$ Topcolor Higgs). Top plot: likelihood function and derived limit for the CCCC subsample. The peak is due to fluctuation of 8 observed events above the expected background of $4.9 \pm 0.5$. Middle plot: likelihood function and derived limit for the CCEC subsample (Luminosity error - the dominant systematics is set to zero), 3 observed events are below predicted background of $5.0 \pm 0.4$; such situation results in the absence of a peak in the likelihood shape; Bottom plot: combined CCCC-CCEC likelihood function and combined limit. The limit improves as a result of combining the two samples via likelihood. 


\begin{tabular}{ccccc}
\hline \multicolumn{5}{c}{ Fermiophobic Higgs } \\
\hline & $\sigma^{95}$ (CCCC) & $\sigma^{95}$ (CCCC(CCEC) & $\sigma^{95}$ combined & $\sigma$ \\
$M_{\mathrm{h}}$ & before $P T^{\gamma \gamma}$ & after $P T^{\gamma \gamma}$ & CCCC and CCEC & NLO theory \\
\hline 60 & $7.86 \mathrm{pb}$ & $2.97(12.49) \mathrm{pb}$ & $3.59 \mathrm{pb}$ & $2.25 \mathrm{pb}$ \\
70 & $7.87 \mathrm{pb}$ & $3.39(5.51) \mathrm{pb}$ & $2.79 \mathrm{pb}$ & $1.53 \mathrm{pb}$ \\
80 & $5.65 \mathrm{pb}$ & $1.63(6.58) \mathrm{pb}$ & $1.71 \mathrm{pb}$ & $1.08 \mathrm{pb}$ \\
90 & $8.86 \mathrm{pb}$ & $1.99(4.45) \mathrm{pb}$ & $1.71 \mathrm{pb}$ & $0.78 \mathrm{pb}$ \\
100 & $2.44 \mathrm{pb}$ & $1.60(2.68) \mathrm{pb}$ & $1.28 \mathrm{pb}$ & $0.58 \mathrm{pb}$ \\
120 & $0.87 \mathrm{pb}$ & $0.70(2.74) \mathrm{pb}$ & $0.70 \mathrm{pb}$ & $0.33 \mathrm{pb}$ \\
\hline \multicolumn{5}{c}{ Topcolor Higgs } \\
\hline \\
$M_{\mathrm{h}}$ & $\sigma^{95}(\mathrm{CCCC})$ & $\sigma^{95}($ CCCC $($ CCEC $))$ & $\sigma^{95}$ combined & $\sigma$ \\
\hline 60 & $8.06 \mathrm{pb}$ & $7.37(33.31) \mathrm{pb}$ & CCCC and CCEC & NLO theory \\
70 & $8.01 \mathrm{pb}$ & $8.22(11.81) \mathrm{pb}$ & $6.08 \mathrm{pb}$ & $6.99 \mathrm{pb}$ \\
80 & $5.60 \mathrm{pb}$ & $3.02(14.37) \mathrm{pb}$ & $3.05 \mathrm{pb}$ & $4.63 \mathrm{pb}$ \\
90 & $9.17 \mathrm{pb}$ & $3.25(8.11) \mathrm{pb}$ & $2.79 \mathrm{pb}$ & $2.22 \mathrm{pb}$ \\
100 & $2.76 \mathrm{pb}$ & $3.92(4.11) \mathrm{pb}$ & $2.66 \mathrm{pb}$ & $1.73 \mathrm{pb}$ \\
120 & $1.04 \mathrm{pb}$ & $0.97(4.05) \mathrm{pb}$ & $0.97 \mathrm{pb}$ & $1.02 \mathrm{pb}$ \\
\hline \multicolumn{5}{c}{}
\end{tabular}

Table 6.1: $95 \% C L$ upper limits on Higgs cross-section. Theoretical cross-sections

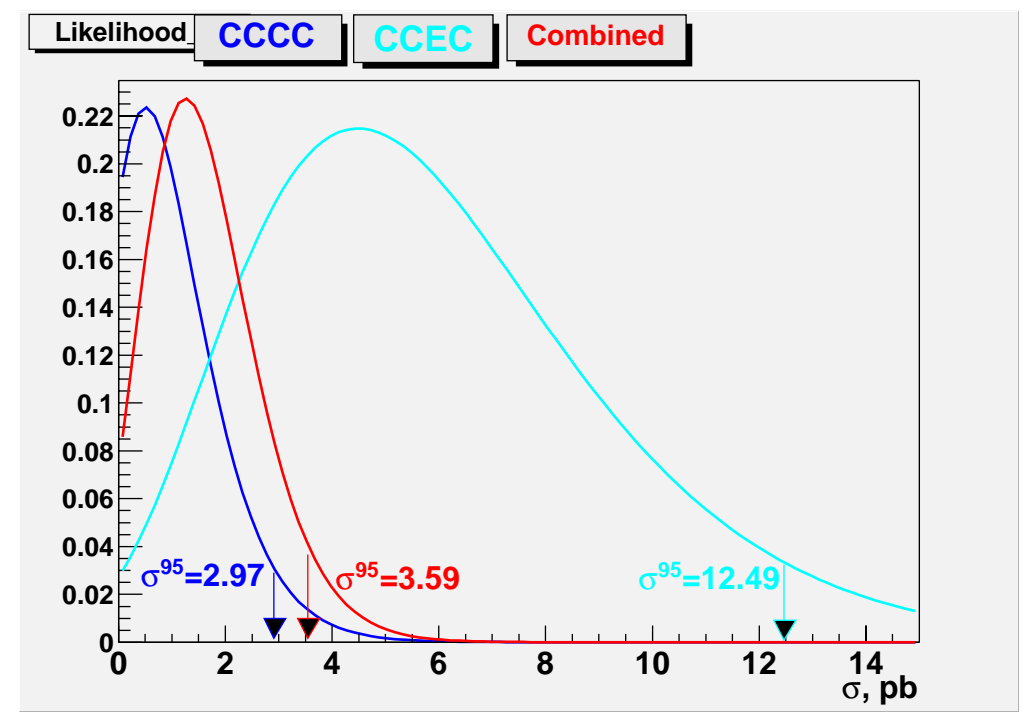

Figure 6.2: Example of worsening of the limit after combining the likelihoods ( $\mathrm{M}=60 \mathrm{GeV}$ Fermiophobic Higgs). Normalization factors were changed for plotting purposes in order that different functions can be overlaid for visual comparison. Left curve: likelihood function and derived limit for the CCCC subsample. Right curve: likelihood function and derived limit for the CCEC subsample (Luminosity error - dominant systematics - is set to zero). Both CCCC and CCEC likelihoods have peaks since number of observed events fluctuates above the background in both cases. The broad shape of the latter corresponds to low acceptance in comparison with the former. Middle curve: combined CCCC-CCEC likelihood function and derived limit. The limit shifts slightly to a higher value. 
In Fig. 6.3 two right columns of Table 6.1 (" $\sigma^{95}$ combined" and " $\sigma$ NLO theory") are plotted.
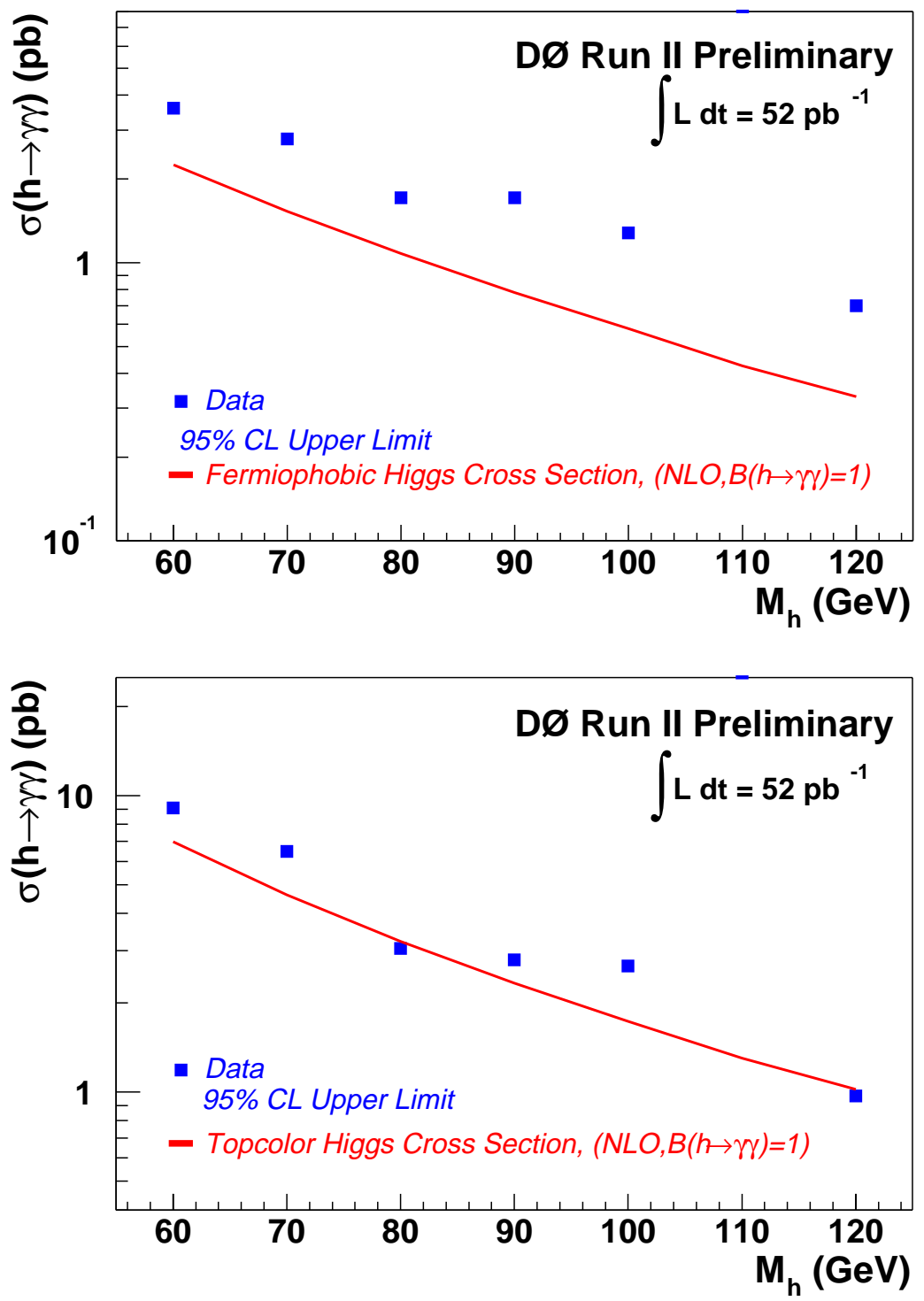

Figure 6.3: 95\% CL cross section limits and NLO theoretical cross sections. Theoretical cross sections are shown with a line; points are experimental limits. Top plot: Fermiophobic Higgs scenario. Bottom plot: Topcolor Higgs scenario.

\subsection{Conclusions}

A search for a non-SM model Higgs with enhanced diphoton decays in the Fermiophobic and Topcolor scenarios was performed in the inclusive $h \rightarrow \gamma \gamma+X$ channel using $51.8 \pm 5.2 \mathrm{pb}^{-1}$ of data collected with the DØ detector between September 2002 and January 2003. Measured $\gamma \gamma$ spectrum is in 
agreement with the Standard Model backgrounds. Kinematic optimization of the analysis was performed. 95\% CL cross section limits for Fermiophobic and Topcolor Higgs for masses of 60, 70, 80, 90, 100, $120 \mathrm{GeV}$ were calculated in the Bayesian approach, assuming the SM Higgs production rate and $\operatorname{Br}(\gamma \gamma)=1$. We are not sensitive yet to the Fermiophobic Higgs signal for given integrated luminosity. In case of the Topcolor scenario we are beginning to be sensitive to the signal just outside the $\mathrm{Z}$ mass peak region. $\mathrm{M}=80 \mathrm{GeV}$ and $\mathrm{M}=120 \mathrm{GeV}$ points can be excluded for the $\gamma \gamma$ branching ratio close to 1. Our current sensitivity is still significantly lower than that in Run I (benchmark Fermiophobic Higgs was excluded at $78.5 \mathrm{GeV}$ (Section 2.4)). Apart from the difference of a factor of 2 in the integrated luminosity, this is a reflection of a high photon misidentification rate that still needs to be understood. This work is currently in progress at DØ . 


\section{Appendix A}

\section{Appendix A}

As described in Section 4.8, we calculate tracking efficiency using the expression $\epsilon($ track $)=\left(2 N_{2}+\right.$ $\left.N_{1}\right) / 2 N_{\text {all }}$, where $N_{2}, N_{1}$ and $N_{\text {all }}$ are obtained from fitting the di-EM mass distribution. A linear function is used to describe the background under the Z-peak.

In order to understand the systematic effects of this measurement we also calculate tracking efficiency using different background fit functions: the polynomial for $\mathrm{CCCC}$ and the exponential for CCEC, ECEC. Corresponding plots are shown in Figures A.1, A.2, and A.3.

Also we calculate efficiencies using the expression $\epsilon($ track $)=\sqrt{N_{2} / N_{\text {all }}}$ The results of these studies are summarized in Table A.

\begin{tabular}{ccc}
\hline bkgd fit & $\epsilon($ track $)=\left(2 N_{2}+N_{1}\right) / 2 N_{\text {all }}$ & $\sqrt{N_{2} / N_{\text {all }}}$ \\
\hline CCCC & $72.7 \pm 0.9 \%$ & $70.7 \pm 1.0 \%$ \\
linear & $74.0 \pm 0.9 \%$ & $72.1 \pm 1.0 \%$ \\
polynom. & & \\
\hline CCEC & $62.6 \pm 1.0 \%$ & $63.9 \pm 1.1 \%$ \\
linear & $61.6 \pm 1.0 \%$ & $63.4 \pm 1.1 \%$ \\
exponent. & & \\
\hline ECEC & $63.7 \pm 2.2 \%$ & $66.8 \pm 2.4 \%$ \\
linear & $59.1 \pm 2.1 \%$ & $63.6 \pm 2.3 \%$ \\
exponent &
\end{tabular}

Table A.1: Comparison of tracking efficiencies obtained via different formulas and different background fit functions. 
We conclude that systematic error due to difference in method is small. For central values we use the numbers obtained with expression $\epsilon($ track $)=\left(2 N_{2}+N_{1}\right) / 2 N_{\text {all }}$ based on a linear background fit. We use the difference in the efficiency obtained via two different formulas (using linear background fit) as an estimate of systematics.
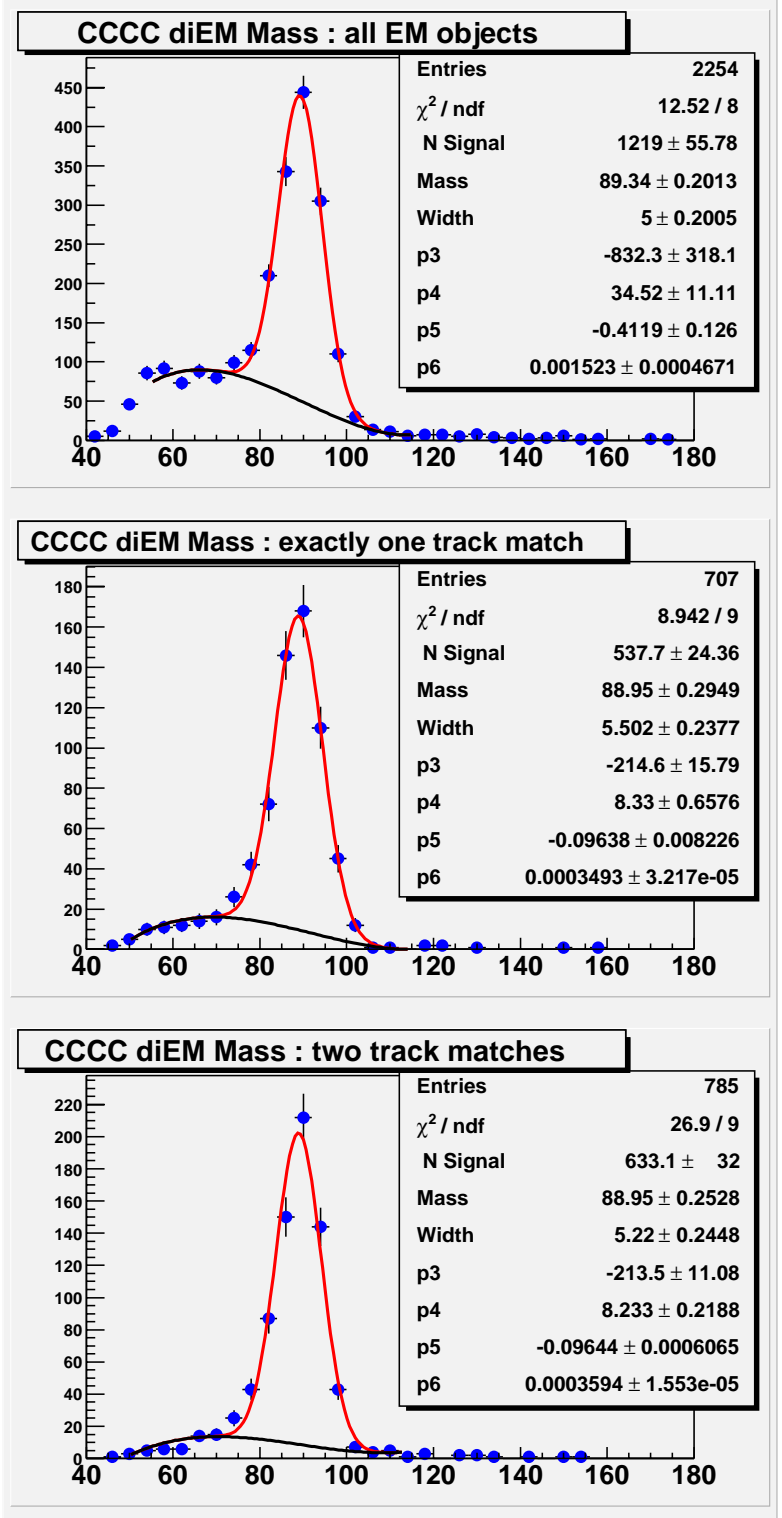

Figure A.1: di-EM mass distributions for Tracking Efficiency measurement for CCCC events. Top plot shows mass spectrum and the fit for the whole sample, middle plot - events with exactly one track match, bottom plot - both EM objects are matched to tracks. 


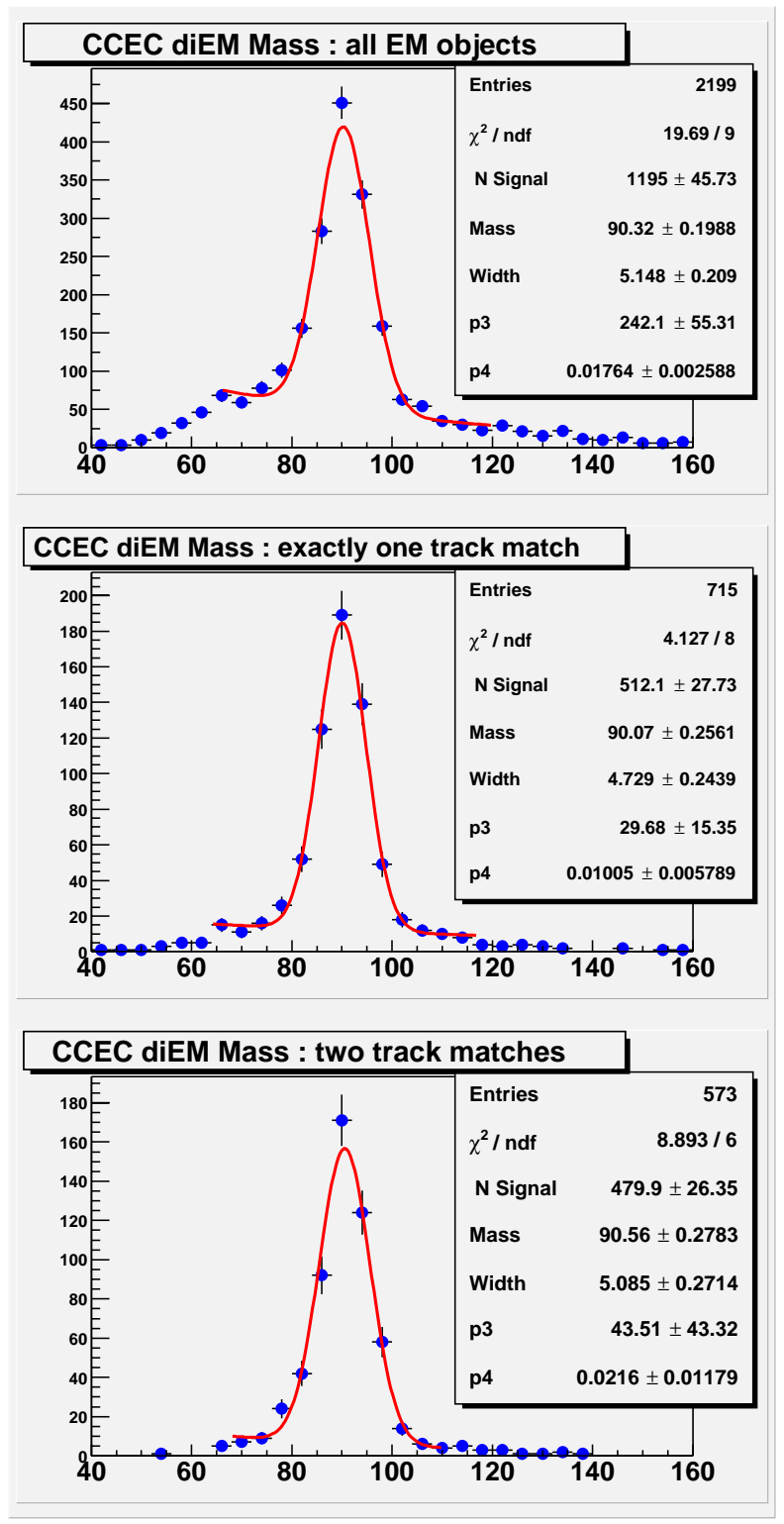

Figure A.2: di-EM mass distributions for Tracking Efficiency measurement for CCEC events. Top plot shows mass spectrum and the fit for the whole sample, middle plot - events with exactly one track match, bottom plot - both EM objects are matched to a tracks. 


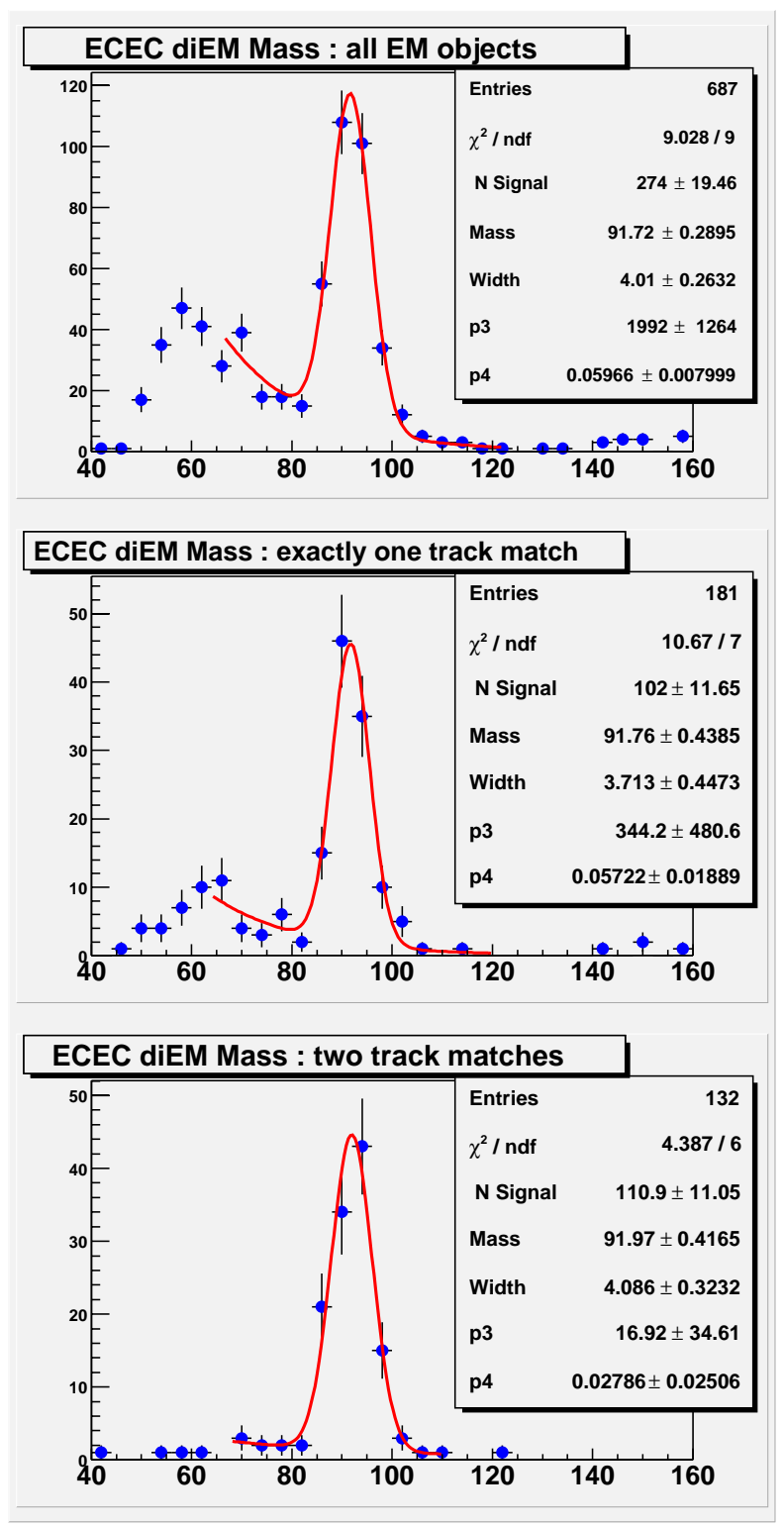

Figure A.3: di-EM mass distributions for Tracking Efficiency measurement for ECEC events. Top plot shows mass spectrum and the fit for the whole sample, middle plot - events with exactly one track match, bottom plot - both EM objects are matched to tracks. 


\section{Bibliography}

[1] J. Bardeen, L.N. Cooper, and J.R.Schrieffer, Phys.Rev. 108, 1175 (1957).

[2] C.A. Coulson and M.J. Kearsley, Proc. Roy. Soc. (London), A241, 433 (1957).

[3] S.L Glashow, Nucl. Phys. 22579 (1961);

S. Weinberg, Phys. Rev. Lett. 191264 (1967);

A. Salam, Elementary Particle Theory, ed. N. Svartholm (Almquist and Wiksells, Stockholm, 1969) p.367.

[4] G. Arnison et al., (UA1 Collaboration), Phys. Lett. B126 398 (1983);

Phys. Lett. B129 273 (1983);

[5] P. Bagnaia et al., (UA2 Collaboration), Phys. Lett. B129 310 (1983);

Z. Phys. C24 1 (1984).

[6] G. Landsberg, Ph.D Thesis, SUNY at Stony Brook, (1994) (Unpublished).

[7] B. Abachi et al. (DØ Collaboration), Phys. Rev. Lett. 742632 (1995).

[8] F.Abe et al. (CDF Collaboration), Phys. Rev. 74, 2626 (1995).

[9] V. Bargmann and E.P. Wigner, Proc. Nat. Acad. Sci. (USA) 34211 (1948)

[10] S.W. Hawking, A Brief History of Time, Bantam Books, 1988. 
[11] P.W. Higgs, Phys. Rev. Lett. 12508 (1964);

Phys Rev. 1451156 (1966);

F. Englert and R. Brout, Phys. Rev. Lett. 13321 (1964);

G.S. Guralnik, C.R. Hagen, and T.W.B. Kibble, Phys. Rev. Lett. 13585 (1964);

T.W.B. Kibble, Phys. Rev. 1551554 (1967).

[12] R. Genik II, Ph.D Thesis, Michigan State University (1998) (Unpublished).

[13] M. Kaku, Quantum Field Theory, Oxford University Press, 1993.

[14] G. Kane, Modern Elementary Particle Physics, Updated Edition, Perseus Books, Reading Massachusetts, 1993, p.100.

[15] D. Griffiths, Introduction to Elementary Particles, John Wiley \& Sons,Inc, 1987, p.348.

[16] V.D. Barger and R.J.N. Phillips, Collider Physics, Addison-Wesley, 1988.

[17] H. Baer et al., Low Energy Supersymmetry Phenomenology, hep-ph/9503479.

[18] B. Lee, C. Quigg, and H. Thacker, Phys Rev. D16 (1977).

[19] S. Dawson, hep-ph/9612229, 1997.

[20] J. Wess and J. Bagger, Supersymmetry and Supergravity, (Princeton University Press, Princeton, N.J. 1983);

P. Fayet and S. Ferrara Phys. Rep. 32249 (1977).

[21] J.F. Gunion et al., it The Higgs Hunter's Guide, (Perseus Publishing,Cambridge, Massachusetts, 1990) p.339.

[22] Review of Particle Physics, Part 1, PDG, Phys. Rev. D66 010001-98, 010001-89, 010001-271 (2002).

[23] M. Carena et al., hep-ph/0010338.

[24] HIGLU, VV2H, V2HV Fortran programs by M. Spira. 
[25] Comput. Phys. Commun. 10856 (1998).

[26] S. Mrenna, J. Wells, Phys. Rev. D63, 015006 (2001).

[27] H.E. Haber, G.L. Kane, and T. Sterling, The Fermion Mass Scale and Possible Effects of Higgs Bosons on Experimental Observables Nucl., Phys. B161 493 (1979);

J.F. Gunion et al., Phys. Rev. D43, 1673 (1990);

B. Grzadkowski and J.F.Gunion, hep-ph/9910456.

[28] H. Baer and J.D. Wells Trilepton Higgs signal at hadron colliders, Phys. Rev. D57, 4446 (1997).

[29] W. Loinaz and J.D. Wells, Phys. Lett. B445 178 (1998).

[30] M. Carena et al., Phys. Rev. D60, 076010 (1999).

[31] M. Carena et al., hep-ph/9907422.

[32] L.J. Hall et al., Phys. Rev. D50, 7048 (1994).

[33] J.D. Wells, Phys. Rev. D56, 1504 (1997).

[34] C.T. Hill, Phys. Lett. B266, 1419 (1991);

C.T. Hill, Phys. Lett. B345, 483 (1995).

[35] G. Landsberg, K. Matchev, Phys. Rev. D62, 035004 (2000).

[36] B. Dobrescu, hep-ph/9908391; B. Dobrescu, G. Landsberg, and K. Matchev, Phys. Rev. D63, 0075003 (2001).

[37] L. Hall and C. Kolda, Phys. Lett. B459, 213 (1999);

H. Cheng et al., hep-ph/9912343.

[38] B. Abbot et al. (DØ Collaboration), Phys Rev. Lett. 82, 2244 (1999).

[39] Brian Lauer,Ph.D Thesis, Iowa State University (1997) (Unpublished).

[40] F.Abe et al., (CDF Collaboration), Phys. Rev. D59, 092002 (1999). 
[41] A. Rosca, hep-ex/0212038 (2002).

[42] http://lephiggs.web.cern.ch/LEPHIGGS/talks/lepsem220702.pdf

[43] http://www.fnal.gov/pub/about/whatis/history.html

[44] R.R. Wilson, Starting Fermilab, http://www.fnal.gov/projects/history/wilson/cover.html

[45] P. Demine, Ph.D. Thesis, Universite Joseph Fourier - Grenoble 1 - U.F.R. de Physique, (2002) (Unpublished).

[46] Abid M. Patwa, Ph.D. Thesis, SUNY at Stony Brook (2002) (Unpublished).

[47] Michele Petteni, Ph.D Thesis, Imperial College, London, (2003).

[48] http://www.totse.com/en/fringe/fringe_science/tevatron.html

[49] B. Abachi et al., (DØ Collaboration), Nucl. Instr. and Methods A338 185 (1994).

[50] A.R. Baden, Jets and Kinematics in Hadronic Collisions, January 1997

[51] http://www-d0.fnal.gov/runcoor/DAQ/Tutorials/2003/2003-02-18_Kajfasz_SMT-01.ppt, http://www-d0.fnal.gov/runcoor/DAQ/Tutorials/2003/2003-02-18_Kajfasz_SMT-02.ppt

[52] DØ Silicon Tracker Technical Design Report, DØ note 2169.

[53] The DØ Upgrade. The Detector and Its Physics, the DØ Collaboration, July 30, 1996.

[54] "Silicon Detector", talk by R. Lipton at Fermilab Lecture Series.

[55] "Collider Detectors and Collider Physics", talk by John Womersley at Fermilab Summer Lecture Series.

[56] DØ Collaboration, Calorimeter Electronics Upgrade for Run2, Technical Design Report, V1.7, May 6, 1998;

http://www-d0.fnal.gov/ d0upgrad/calelec/intro/tdr/tdr17.pdf

[57] http://hep.brown.edu/users/SiDet/welcome.html 
[58] D. Adams et al., Fermilab-Conf-94/318-E, presented at DPF'94

[59] R. Fruhwirth et al., it Data Analysis Techniques for High-Energy Physics, second edition (Cambridge University Press, 2000) pp.185, 186, 188, 192, 194-210.

[60] I.S. Hughes, it Elementary Particles, second edition (Cambridge University Press, 1985) p31.

[61] B.R. Martin, G. Shaw, it Particle Physics, (John Wiley \& Sons, 1992) p54.

[62] R. Fernow, it Introduction to xperimental particle physics, (Cambridge University Press, 1992) p274.

[63] Techniques and Concepts of High-Energy Physics III, edited by Thomas Ferbel, NATO ASI Series, Series B: Physics Vol. 128, pp.281-336.

[64] C. Fabjan and T. Lundlam, Calorimetry in high energy physics, Ann. Rev. Nucl. Part. Sci., 32: 335-89, 1982.

[65] H. Weidkamp, DiplomArbeit, Rhein-Westf Tech. Hochschule Aachen (1984).

[66] R. Wigmans, Calorimetry: Energy Measurement in Particle Physics, Clarendon, Oxford (2000).

[67] K. Leroy and P.-G Rancoita, Rep. Prog. Phys 63 (2000) 505;

[68] Design Report, The DØ Experiment at the Fermilab $\bar{p} p$ Collider, November 1984.

[69] W. Guryn, Proceedings of the Second International Conf. on Calorimetry in High Energy Physics, Capri, Italy, October 14-18, 1991.

[70] Manufacturing Sciences Corp., Oak Ridge, TN.

[71] M. Adams et al., Design Report of the Central Preshower Detector for the DØ Upgrade, DØ note 3014 .

[72] http://www-d0.fnal.gov/runcoor/DAQ/2003-01-24_Begel_Luminosity.ps 
[73] C.-C.Miao, R.Partridge, Study of the Run II Luminosity Monitor Counter Design, DØ Internal Note 3319, 1998.

[74] A.Lo,C.-C.Miao, R.Partridge, Luminosity Monitor Technical Design Report, DØ Internal Note $3320,1997$.

[75] C.-C.Miao, the DØ Run II Luminosity Monitor, Nucl. Phys. Proc. Suppl., 78, 342-347 (1999).

[76] A. Brandt et al., A Forward Proton Detector at DØ , FERMILAB-Pub-97/377, 1997.

[77] http://heplab2.phy.uic.edu/physics/work/slides/buehler_physfest_jan23_2003.pdf

[78] http://d0.phys.washington.edu/ haas/d0/13/sci2002.pdf

[79] http://www-d0.fnal.gov/runcoor/DAQ/Tutorials/2003/2003-01-27_Chapin_L3DAQ.pdf

[80] http://www-d0.fnal.gov/d0dist/dist/packages/em_analyze/v01-02-22/doc/EMAnalyze_doc.ps

[81] http://www-d0.fnal.gov/phys_id/emid/d0_private/certification/main_v2_2_1.html

[82] http://www-d0.fnal.gov/phys_id/emid/d0_private/emreco_doc_alex.ppt

[83] http://www-d0.fnal.gov/phys_id/emid/d0_private/minutes/20020327alex.ppt

[84] http://www-d0.fnal.gov/phys_id/emid/d0_private/EM_Particle_Documentation_EMID.html

[85] http://www-

d0.fnal.gov/phys_id/emid/d0_private/certification/v2_2/emid_macros_v2_2_1.html

[86] A. Melnitchouk, "Search for non-SM Light Higgs Boson in the $h \rightarrow \gamma \gamma$ Channel", DØ note 4117, 2003.

[87] http://www-d0.fnal.gov/Run2Physics/np/

[88] http://www.thep.lu.se/ torbjorn/Pythia.html

[89] S.Fu et al., DØ Note 4010 (2002).

[90] 10/23/02 EMID talk by Y. Arnoud. 
[91] ROOT based Poisson statistics limit calculator by Greg Landsberg

[92] http://www-d0.fnal.gov/phys_id/jes/d0_private/certified/v4.2/links.html

[93] G. Landsberg, Private communication;

B. Knuteson, Algorithms for Computing Significance, DØ note 3345, 1997.

[94] Significance computation code by G. Landsberg.

[95] http://dpf2002.velopers.net/talks_pdf/382talk.pdf

[96] Y. Gershtein, private communication.

[97] Martin Ernst Los, Ph.D Thesis, Universiteit van Amsterdam (1995) (Unpublished)

[98] http://www.hep.uiuc.edu/home/catutza/Docs/Root_Jetnet.tar.gz; http://www.hep.uiuc.edu/home/catutza/Docs/RJ_intro.ps.gz

[99] A. Turcot, private communication.

[100] http://www.thep.lu.se/public_html/jetnet_30_manual/

[101] D.S. Sivia, Data Analysis. A Bayesian tutorial, Clarendon Press, Oxford, 1996.

[102] I. Bertram, G.Landsberg, J.Linnemann, R.Partridge, M.Paterno, H.B.Prosper, A Recipe for the Construction of Confidence Limits, DØ note 3476, 1999. 